\title{
40. MAGNETOSTRATIGRAPHY OF NEOGENE AND QUATERNARY SEDIMENT SERIES FROM THE NORWEGIAN SEA: OCEAN DRILLING PROGRAM, LEG 104
}

\author{
Ulrich Bleil ${ }^{2}$
}

\begin{abstract}
Results of a detailed paleomagnetic study on largely undisturbed sedimentary sequences recovered in the Vøring Plateau region of the Norwegian Sea during Ocean Drilling Program Leg 104 are presented. At each drill site an essentially continuous downhole magnetic reversal pattern could be defined to depths between 200 and $300 \mathrm{~m}$ below seafloor allowing correlations with a calibrated geomagnetic time scale and establishing almost complete magnetostratigraphic records for the core material analyzed. A composite section of the drill holes represents the first high-quality chronostratigraphic framework from the lower Miocene through Holocene obtained in the Norwegian Sea. It should provide a basis for first-order correlations with calcareous and siliceous microfossil events and contribute to a further elaboration of the regional paleoceanographic history. A series of major hiatuses in the upper and middle Miocene accounts for about 4 million yr of missing stratigraphic record.
\end{abstract}

\section{INTRODUCTION}

A prime objective of Leg 104 of the Ocean Drilling Program (ODP) was to study the paleoceanographic evolution of the Norwegian Sea, in particular to document the temporal variability of the Norwegian Current which provides northwestern Europe with temperate climates. To this aim a series of seven holes at three sites were drilled into the Cenozoic sedimentary deposits on a transect from near the foot of the Voring Plateau, over the outer Vøring Plateau to the Vøring Basin (Fig. 1, Table 1). This strategy was designed to recover sediment formations from environments ranging from deep ocean basin to the central continental slope with the goal of defining vertical and horizontal gradients associated with the current system.

Systematic paleomagnetic analyses of the core materials were carried out to establish a detailed chronological framework which could be integrated with biostratigraphic, paleoclimatic, tectonic, and other studies. Magnetostratigraphic results presented in this paper are exclusively based on measurements of discrete samples (some of these data have already been reported in the respective site reports; Eldholm, Thiede, Taylor, et al., 1987). Measurements of natural remanent magnetization (NRM) properties of core sections using the new shipboard pass-through cryogenic magnetometer to a large extent were found unsuitable for magnetostratigraphic interpretations and therefore will not be discussed in detail.

The coring operations were accomplished with the advanced hydraulic piston corer (APC) and below APC refusal in the deeper, more consolidated formations, pursued with the extended core barrel $(\mathrm{XCB})$ drilling technique. The core material recovered from the upper 200 to $300 \mathrm{~m}$ of the sediment column in general proved well-suited to magnetostratigraphic work. Below this depth the recovery deteriorated drastically in quality and quantity. Unlike in previous DSDP operations, the collection of duplicate HPC cores was only of limited value in achieving continuous records of the sediment accumulation in composite sections.

\footnotetext{
${ }^{1}$ Eldholm, O., Thiede, J., Taylor, E., et al., 1989. Proc. ODP, Sci. Results, 104: College Station, TX (Ocean Drilling Program).

2 Fachbereich Geowissenschaften, Universität Bremen, Postfach 330 440, 2800 Bremen 33, Federal Republic of Germany.
}

The polarity time scale of Berggren et al. (1987) is used throughout this study. The nomenclature for Cenozoic geomagnetic time units follows the new chron scheme proposed by LaBrecque et al. (1983), which links the geomagnetic chrons directly to the numbered and lettered sequence of positive marine magnetic anomalies. By their definition each " $\mathrm{C}$ " chron is composed of a younger predominantly normal interval corresponding to the magnetic anomaly as well as the preceding predominantly reversed interval. The details of this terminology are shown in Figure 2. For Pleistocene and Pliocene times the widely adopted Brunhes, Matuyama, Gauss, and Gilbert geomagnetic chron scheme is also used in the text together with the traditional names of their various short polarity events.

\section{METHODS AND MATERIALS}

Sampling intervals varied between 30 and $50 \mathrm{~cm}$ ( 3 to 5 specimens per core section) in the first APC/XCB hole of each site and between 50 and $70 \mathrm{~cm}$ ( 2 or 3 specimens) in the second hole. Depending on the apparent sedimentation rates encountered, the sample spacing represents time intervals ranging from $10^{4} \mathrm{yr}$ or less to, in certain intervals, almost $10^{5} \mathrm{yr}$. The individual cubic specimen of about $2 \mathrm{~cm}$ on edge therefore comprises a paleomagnetic record of several hundred to several thousand years. The results show that the sampling scheme was generally adequate to identify most of the reversal history of the Earth's magnetic field in the remanent magnetization logs of the sediment series penetrated and only few of the most fine-scale structures could not be resolved where the biostratigraphies indicated a continuous accumulation.

During ODP Leg 104 the new pass-through cryogenic magnetometer was available for shipboard paleomagnetic measurements. On virtually all sediment core sections the natural remanent magnetization properties were determined with this ingenious instrumentation. Although its performance was excellent in most every respect during the entire cruise, the results obtained were altogether disappointing. The almost continuous NRM inclination logs show a core to core variability, and over extended intervals of all holes, a pervasive degree of overprinting by normal secondary magnetization components that masks the primary remanent polarity pattern. A comparison of shipboard NRM inclination measurements on the archive split-halves of core sections with shorebased NRM data of discrete samples taken at about the core axis of the working split-halves (Figs. 3, 4) indicates that this effect is apparently strongest in the outer parts of the core. Outer parts make up a comparatively large portion of the core volume. This implies that the secondary spurious magnetization of normal polarity has been acquired during the coring process or the subsequent core handling and is not of in-situ viscous origin. As an alternating field demagnetization device for whole 


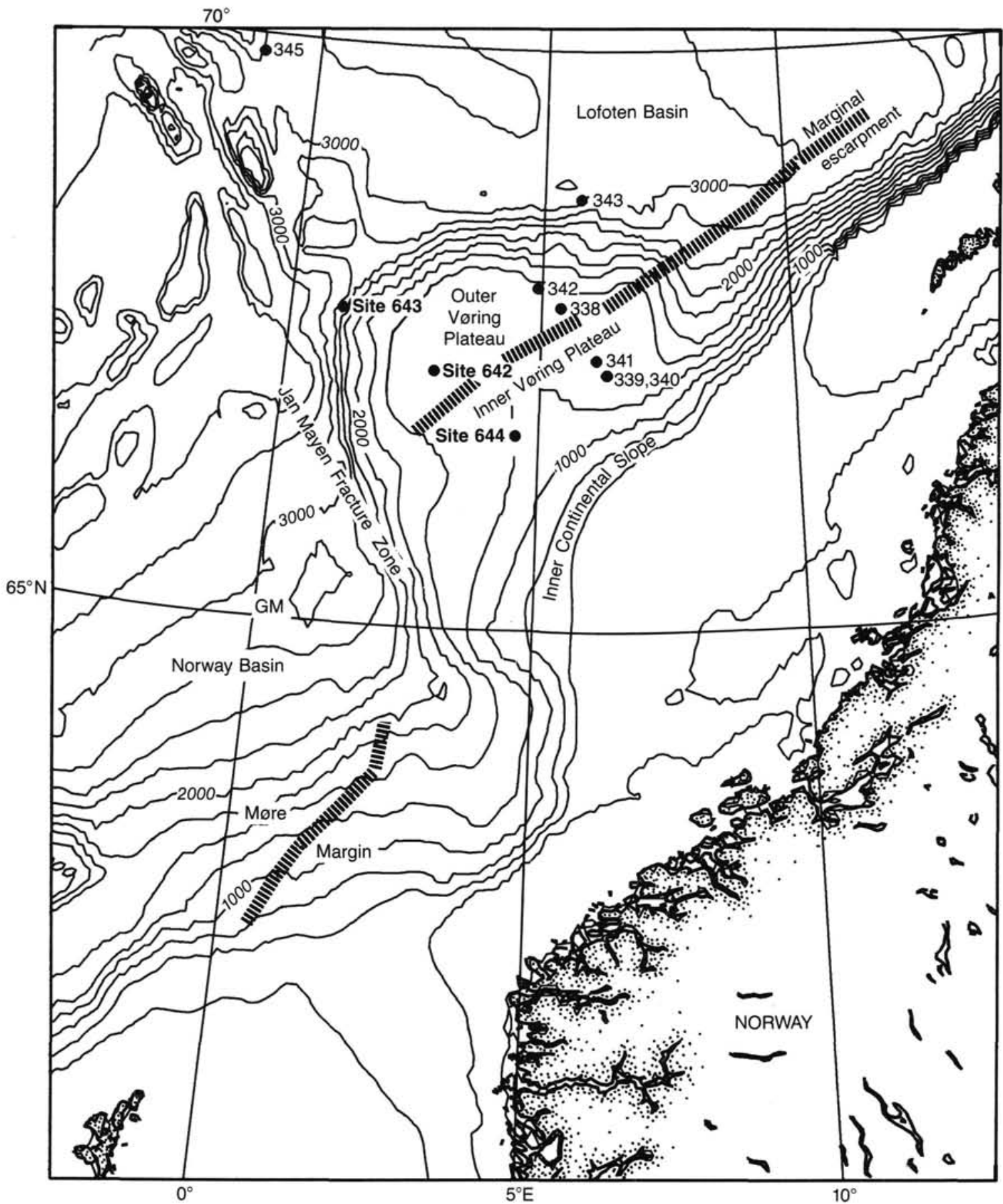

Figure 1. Locations of ODP Leg 104 drill sites (642-644) in the Vøring Plateau region of the Norwegian Sea. Contour interval, $250 \mathrm{~m}$.

core sections was not yet operational during ODP Leg 104, no attempt could be made to isolate the stable magnetization components on board.

On these grounds it was only possible to decipher fairly plausible magnetostratigraphies from the shipboard NRM inclination data in the upper parts of each hole where the NRM intensities were typically of the order of some $10^{-2} \mathrm{~A} / \mathrm{m}$. Even there, a tentative interpretation of the paleomagnetic records had to rely largely on the general age framework of paleontological zonations. Figures 3 and 4 illustrate the rather dramatic variability in shipboard NRM data quality in two consecutive cores retrieved from the same lithologic unit. While in Core 104-644A$11 \mathrm{H}$ the shipboard inclination log suggests the possible recording of an interval with frequent reversals, the NRM data from discrete samples yield predominantly reversed polarities. After a detailed AF demagnet- ization treatment all characteristic stable inclinations are clearly negative. In contrast, in the following Core $104-644 \mathrm{~A}-12 \mathrm{H}$ the normal polarity Jaramillo Subchron in the late part of the reversed Matuyama Chron is almost as precisely defined in the shipboard NRM data as from complete shore-based paleomagnetic analyses of discrete samples. This is a rare exception, though.

A total of more than 2,500 samples have been analyzed for their paleomagnetic properties. See Appendix tables. Measurements of magnetization intensity and direction were made on a 3-axis cryogenic magnetometer (Cryogenic Consultants, Model GM 400). A Schonstedt GSD-1 single-axis demagnetizer was used for the alternating field demagnetization.

In addition to measurements of the NRM, systematic step-wise demagnetization treatments were carried out on each sample. For the bulk 
Table 1. Location of sites drilled on ODP Leg 104

\begin{tabular}{lccc}
\hline Hole & $\begin{array}{c}\text { Latitude } \\
(\mathrm{N})\end{array}$ & $\begin{array}{c}\text { Longitude } \\
(\mathrm{E})\end{array}$ & $\begin{array}{c}\text { Water depth } \\
(\mathrm{m})\end{array}$ \\
\hline $642 \mathrm{~A}, \mathrm{~B}$ & $67^{\circ} 13.5^{\prime}$ & $02^{\circ} 55.7^{\prime}$ & 1286 \\
$642 \mathrm{C}$ & $67^{\circ} 13.2^{\prime}$ & $02^{\circ} 55.8^{\prime}$ & 1277 \\
$642 \mathrm{D}$ & $67^{\circ} 13.2^{\prime}$ & $02^{\circ} 55.8^{\prime}$ & 1272 \\
$643 \mathrm{~A}$ & $67^{\circ} 42.9^{\prime}$ & $01^{\circ} 02.0^{\prime}$ & 2753 \\
$644 \mathrm{~A}, \mathrm{~B}$ & $66^{\circ} 40.7^{\prime}$ & $04^{\circ} 34.6^{\prime}$ & 1227 \\
\hline
\end{tabular}

of the material, the demagnetization was taken to the $50-\mathrm{mT}$ level, which generally exceeds the median destructive field. Steps of 2.5 and 5 $\mathrm{mT}$ were typically applied up to $10 \mathrm{mT}$, followed by $5-\mathrm{mT}$ steps up to 20 or $30 \mathrm{mT}$ and $10-\mathrm{mT}$ steps beyond that stage. Characteristic stable magnetization directions were evaluated from different graphic representations of the demagnetization data. This method has been successfully applied during previous paleomagnetic work on DSDP sedimentary series and a detailed description was given in the respective DSDP Initial Reports (e.g. Bleil, 1985).

With regard to their responses to progressive AF demagnetization, three general types of magnetization structures and stabilities were encountered in the sediments of the Vøring Plateau region:

\section{A. Single-component Remanence}

This is an essentially single-component remanence with a (very) minor overprint of a normal polarity which is likely to represent an in-situ viscous magnetization and is entirely removed after demagnetization to $10 \mathrm{mT}$ or less (Fig. 5). These features characterize a large number of samples from the upper sediment column of all holes with NRM intensities of the order of $10^{-2} \mathrm{~A} / \mathrm{m}$ and median destructive fields typically higher than about $15 \mathrm{mT}$. In the deeper parts of the holes this type is still relatively common in biosiliceous sediments.

\section{B. Two-component Remanence}

This is a two-component remanence with a strong spurious steep inclination normal polarity component that was probably acquired during coring (Fig. 6). Frequently, alternating field demagnetization to several tens of $\mathrm{mT}$ was necessary to isolate the primary stable component. Samples showing this behavior were found at almost all stratigraphic levels regardless of the respective lithology.

\section{Multicomponent Remanence}

This is a multicomponent remanence yielding usable polarity information after a systematic demagnetization treatment (Fig. 7). Despite an often poor directional stability the demagnetization data are considered sufficiently clear to define an unequivocal polarity. In particular, in the lower parts of all holes, a large number of specimens fell into this class where the NRM intensities are of the order of $10^{-4} \mathrm{~A} / \mathrm{m}$.

Type B and C remanences are present in more than $50 \%$ of all samples analyzed. Note therefore that in the present study the widely used pilot-sample demagnetization method would not have been adequate to define acceptable magnetostratigraphic records because a single-step demagnetization at 10 or $20 \mathrm{mT}$ frequently yields a different polarity than a detailed demagnetization procedure (i.e., Fig. 3).

Absolute azimuthal or even relative azimuthal orientation between cores is not available for any of the Leg 104 drill holes. Previous poor experience was repeated again as the Eastman multishot system data were once more found to be basically useless for this purpose. However, the steep geomagnetic field inclinations of the present latitudes and predicted for the paleolatitudes of all sites allow an unambiguous determination of polarities, despite the lack of declination control.

\section{RESULTS}

\section{Site 642}

At Site 642 on the outer Vøring Plateau a total of four holes were drilled into predominantly pelagic and hemipelagic upper Cenozoic sediments overlying a thick volcanic sequence of $\mathrm{Pa}-$ leogene seaward-dipping seismic reflectors. Hole $642 \mathrm{~A}$ was an APC test hole from which only a single core was retrieved; it ap- parently did not contain the uppermost sediments. In the two adjacent Holes 642B and 642C, Neogene and Quaternary sedimentary series were recovered in stratigraphic duplication using the APC system to refusal at around 200 meters below sea floor (mbsf). From this depth the extended core barrel (XCB) drilling technique was applied in Hole 642D to penetrate the sediment column down to the basalt contact at about 330 mbsf.

\section{Hole $642 A$}

Paleomagnetic analyses of samples from the single core obtained in Hole 642A yielded almost exclusively normal polarities. These glacial marine deposits apparently accumulated during the present Brunhes geomagnetic Chron, and the base of the core should have an age of less than $0.73 \mathrm{Ma}$.

\section{Holes $642 B$ and $642 C$}

In the two adjacent Holes $642 \mathrm{~B}$ and $642 \mathrm{C}$, the upper Pliocene through Holocene sediments of lithologic Unit I are composed of interbedded, dark, carbonate-poor, glacial muds and light, carbonate-rich, interglacial marine sandy muds. Lithologic Unit II extends from about 60 to $157 \mathrm{mbsf}$ and was divided into four subunits composed of nannofossil oozes with minor diatom-nannofossil oozes and muds (60-83 mbsf), siliceous muds and oozes (83-108 mbsf), interbedded nannofossil oozes, siliceous nannofossil oozes, and siliceous muds and oozes (108$146 \mathrm{mbsf}$ ), and mixed siliceous-calcareous oozes with minor siliceous muds and nannofossil oozes (146-157 mbsf). Lithologic Unit III consists of siliceous muds and oozes. The Pliocene/Miocene boundary was identified at about 97 mbsf, the upper/ middle Miocene boundary at about $200 \mathrm{mbsf}$. Both holes had to be abandoned due to refusal of the advanced piston corer in middle Miocene sediments.

Throughout Hole 642B the paleomagnetic sampling scheme was as detailed as possible with, on average, one sample taken every $30 \mathrm{~cm}$. In Hole $642 \mathrm{C}$, which was drilled with the principal objective to fill the stratigraphic gaps in the sequence recovered in Hole 642B, only two samples per core section were analyzed. The downhole variations in natural remanent intensity and stable inclination together with the derived polarity logs are shown in Figures 8 and 9, respectively. Details of the magnetostratigraphic interpretation are given in Tables 2 and 3 and summarized as sedimentation rate curves in Figure 14.

From the top to about 64.7 mbsf, paleomagnetic measurements of Hole 642B sediments revealed a recording of the complete and apparently continuous reversal sequence of the geomagnetic Brunhes and Matuyama Chrons including all major polarity events. An identical magnetostratigraphic section was obtained in Hole $642 \mathrm{C}$ for this interval. However, a comparison of the depth positions of individual reversal boundaries shows variable degrees of mutual offsets amounting up to $6 \mathrm{~m}$ downhole. Although this could effectively be the result of real fluctuations in paleotopography, it is perhaps best explained by the standard ODP procedure to exclusively account for any material loss at the base of each cored interval.

Down to the Matuyama/Gauss boundary the sedimentation rate in Hole 642B amounts to $26.2 \mathrm{~m} / \mathrm{m}$.y.. Within the Brunhes Chron four individual samples gave clearly negative stable inclinations. In addition, in one short interval the stable inclinations were unusually shallow (Fig. 8). Interpreted as true features (events or excursions) of the Earth's field, their ages are interpolated to about $30,000,50,000,150,000,240,000$, and 360,000 $\mathrm{yr}$, respectively. None of these potential geomagnetic events could be identified in the Hole $642 \mathrm{C}$ record, possibly because of the less-detailed sampling scheme. Instead, two negative inclinations were encountered here at greater depths with interpolated ages of 520,000 and $560,000 \mathrm{yr}$, respectively. As an accidental misorientation of individual samples cannot totally be 
U. BLEIL

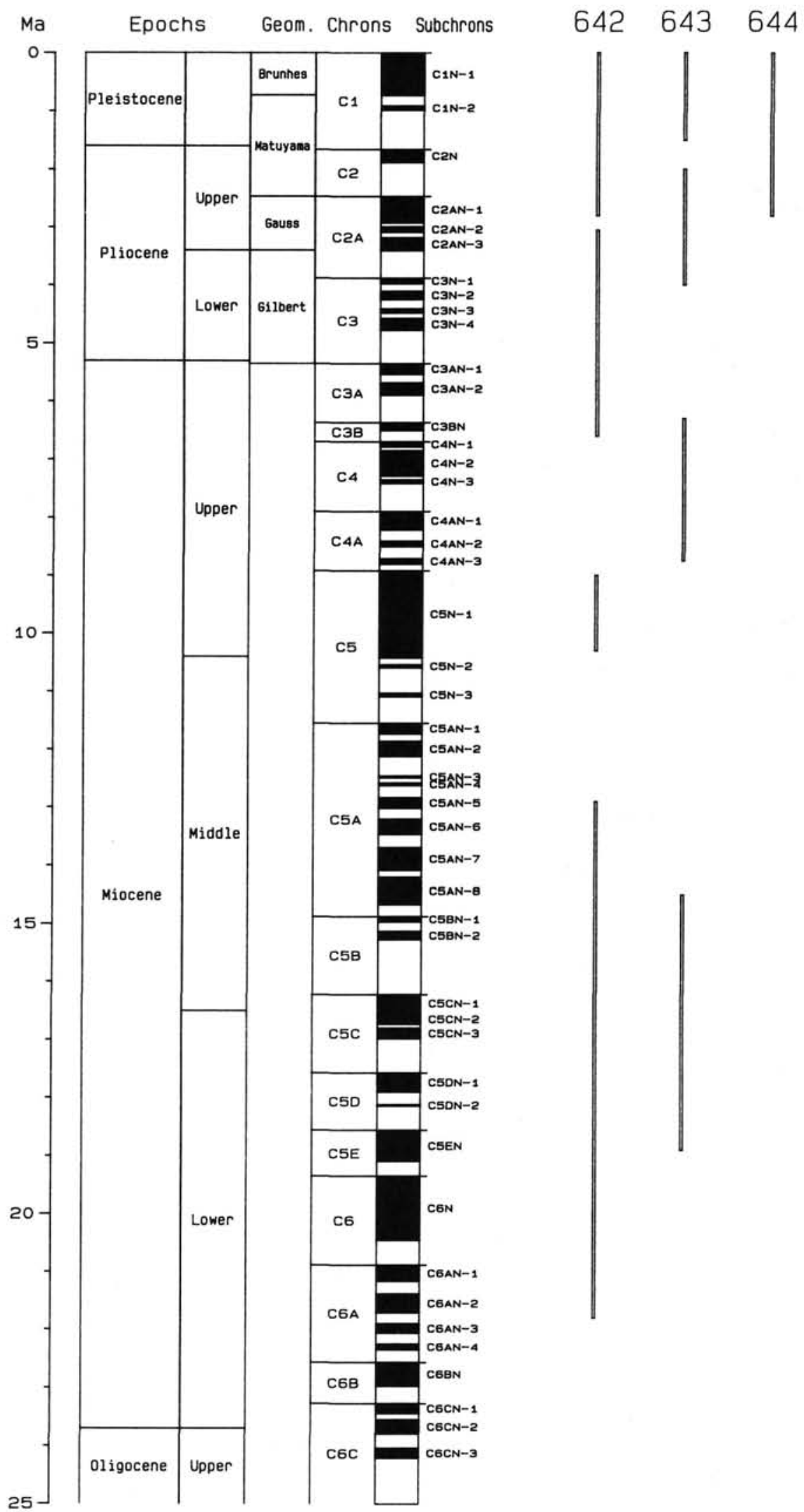

Figure 2. Magnetochronologic scheme used in this study. The geomagnetic polarity time scale (black denoting a normal, white a reversed polarity) based on marine magnetic anomaly lineations is that of Berggren et al. (1987), the magnetic chron nomenclature is that of LaBrecque et al. (1983). Columns on the right indicate the stratigraphic extent of reversal sequences identified in the sediment series recovered at the individual drill sites of ODP Leg 104. 

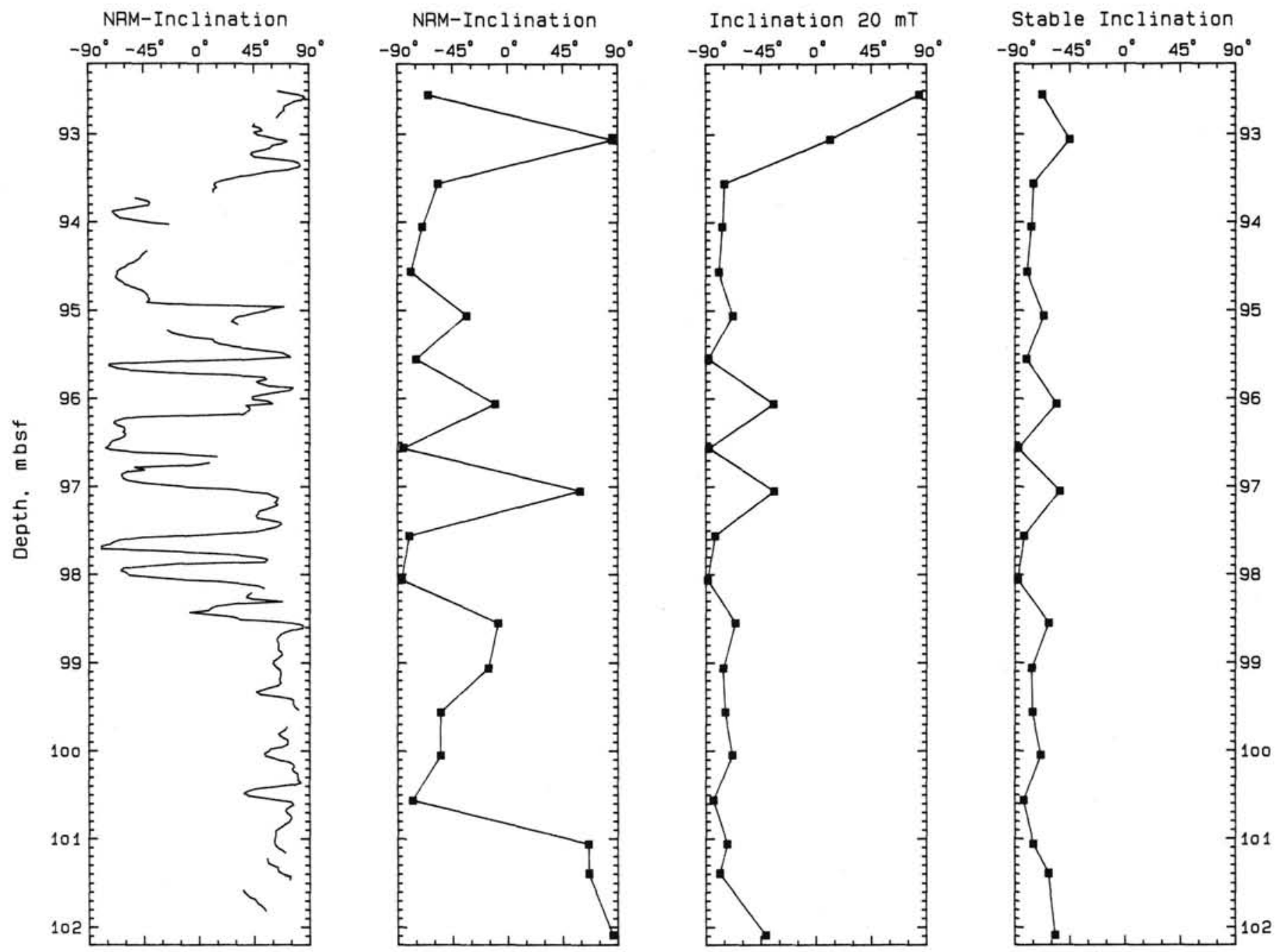

Figure 3. Core 104-644A-11H. Comparison of the natural remanent magnetization (NRM) inclination record obtained from the shipboard passthrough cryogenic magnetometer on split-halves of core sections, with results of shore-based measurements of discrete samples which also include inclinations after $20 \mathrm{mT}$ AF demagnetization and stable inclinations. Minor gaps in the shipboard data occur at section boundaries and in intervals of discontinuous recovery. Note that the sediments of Core 104-644A-11 H accumulated during the late part of the reversed Matuyama Chron between the Jaramillo Subchron and the Brunhes/Matuyama boundary (see Fig. 12).

excluded, and also because of the uncertainties in depth positions mentioned above, at present these results may only be acceptable as general indications for the existence of field reversals or major excursions during the Brunhes Epoch and crude estimates of their timing (see Site 644 results).

Note that in the early part of the Matuyama Epoch the Réunion Event is seen in the paleomagnetic record of Hole 642B.

Because a hiatus of estimated $0.5-\mathrm{m} . \mathrm{y}$. duration was identified near the Cores 104-642B-8H/9H boundary (Spiegler and Jansen, this volume) parts of the Gauss Chron are missing. On the other hand, all four normal polarity events in the reversed Gilbert Chron were identified (Fig. 8). The Gauss/Gilbert boundary (C2AN-3/C2AR-3 transition at $3.40 \mathrm{Ma}$ ) is observed at about 70.0 mbsf. As correlated by Berggren et al. (1987), it approximates the lower/upper Pliocene boundary. The base of the Gilbert Epoch (C3R-4/C3AN-1 transition at $5.35 \mathrm{Ma}$ ) is recognized at about $97.3 \mathrm{mbsf}$ and almost coincides with the Pliocene/Miocene boundary. From the Matuyama/Gauss boundary to this depth the sediments accumulated at an average apparent rate of $11.3 \mathrm{~m} / \mathrm{m}$.y. without accounting for the minor hiatus in the Gauss Chron. With the broad sampling scheme in Hole $642 \mathrm{C}$, the paleomagnetic record is more fragmentary in this in- terval and an interpretation further hampered by an extremely limited recovery in Core 104-642C-10H (Fig. 9).

The upper Miocene paleomagnetic record ends at an unconformity seen in the radiolarian data just below $120 \mathrm{mbsf}$ (Goll and Bjørklund, this volume). The reversal pattern to this level in Hole 642B is incomplete because the recovery in Core 104-642B$14 \mathrm{H}$ is inadequate for paleomagnetic analyses. Despite the fact that a similar situation occurs in Core 104-642C-13H, the sedimentary sequence retrieved here contains all polarity transitions between the top of Chron C3A and the middle of Chron C3B (6.50 Ma, $120.01 \mathrm{mbsf})$. On average, sedimentation between the Pliocene/Miocene boundary and the youngest prominent hiatus at Site 642 took place at an apparent rate of $22.4 \mathrm{~m} / \mathrm{m}$.y.

For the lower part of the upper Miocene and also parts of the middle Miocene the polarity patterns of Holes $642 \mathrm{~B} / \mathrm{C}$ unfortunately do not offer any resolution at all. This is likely to be caused by a combination of different effects. However, the available evidence is insufficient to quantify the various factors. Thus the sediments of Cores 104-642B-15H through 104-642B$20 \mathrm{H}$ (Cores $104-642 \mathrm{C}-16 \mathrm{H}$ through $104-642 \mathrm{C}-21 \mathrm{H}$, respectively) which accumulated during this period carry an almost continuous uniform normal polarity. At least in the $\sim 40$-m interval 

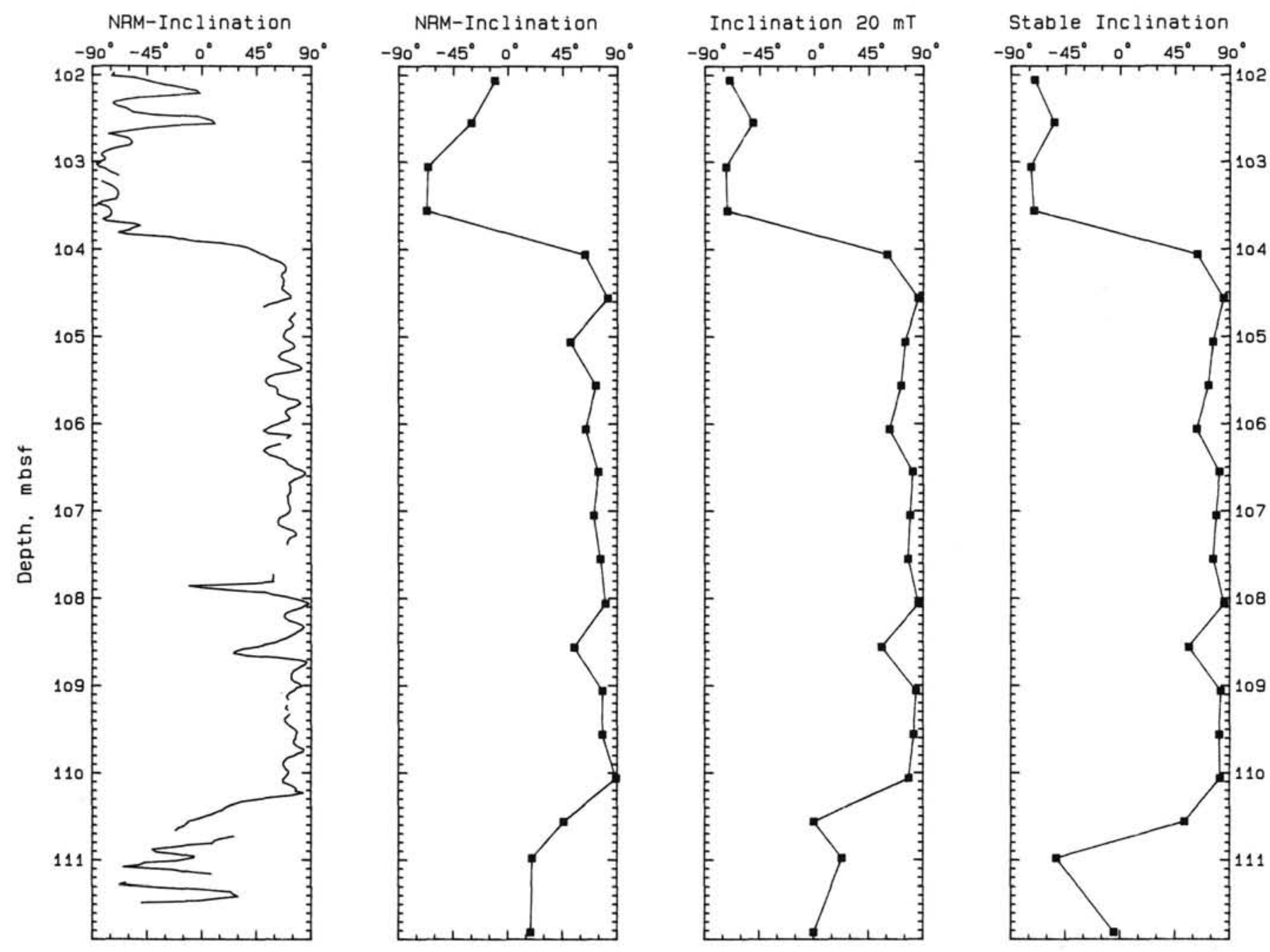

Figure 4. Core 104-644A-12H. Comparison of the natural remanent magnetization (NRM) inclination record obtained from the shipboard passthrough cryogenic magnetometer on split-halves of core sections with results of shorebased measurements of discrete samples which also include inclinations after $20 \mathrm{mT}$ AF demagnetization and stable inclinations. Minor gaps in the shipboard data occur at section boundaries and in intervals of discontinuous recovery. The polarity transition from reversed to normal at around 111 mbsf marks the onset of the Jaramillo Subchron some $0.98 \mathrm{~m}$.y. ago. To document the fine structure of its termination $(0.92 \mathrm{Ma})$ recorded at about $104 \mathrm{mbsf}$, additional closely spaced shore-based sampling was done.

bound between the unconformity at around 120 mbsf mentioned above and the second major hiatus documented at about 160 mbsf by different biostratigraphic methods, a normal magnetization overprint which could not be removed with AF demagnetization techniques appears to obscure the paleomagnetic record. This presumption is based on the fact that none of the numerous reversed $\mathrm{C} 4$ and $\mathrm{C} 4 \mathrm{~A}$ subchrons could be identified. For the lower $\sim 20 \mathrm{~m}$ of this interval the Bolboforma stratigraphy (Qvale and Spiegler, this volume) indicates sediment ages corresponding to the long normal period of Subchron C5N-1 (marine magnetic anomaly $5 ; 8.92$ to $10.42 \mathrm{Ma}$ ). It is interesting to note, therefore, that several features in the respective magnetostratigraphic records (Figs. 8, 9) could be interpreted as short reversed polarity events within the $\mathrm{C} 5 \mathrm{~N}-1$ Subchron. Similar observations have repeatedly been reported in the literature (e.g., McDougall et al., 1976; LaBrecque et al., 1977).

The transition between Subchrons C5AN-5/C5AR-5 (13.01 $\mathrm{Ma}$ ) is tentatively placed at about $177.4 \mathrm{mbsf}$ in Hole $642 \mathrm{C}$. A total of $6.5 \mathrm{~m}$.y. would then be contained between this horizon and the last identified reversal boundary above the first major unconformity. Of course no plausible sedimentation rate can be calculated from the paleomagnetic data for this upper-middle Miocene period nor is it possible to estimate the amount of time spanned by any of the two hiatuses.

According to the radiolarian biostratigraphy (Goll and Bjørklund, this volume), the lower Miocene part of the sedimentary record at Site 642 is disrupted by an additional hiatus at around 213 mbsf (Fig. 14). In the paleomagnetic data there is little supporting evidence for this. The unconformity falls into an interval of low sedimentation rate, in particular, if compared to the situation at Site 643 (see below). This level could therefore be equally well interpreted as representing a condensed zone. In any case, this hiatus appears to span only a short period of time. Although all the details of the early middle Miocene and late lower Miocene Earth's magnetic field reversal history could be resolved in both holes, their combined polarity pattern is complete enough to allow an unequivocal correlation with a calibrated time scale (Tables 2, 3; Fig. 14). At the base in Hole $642 \mathrm{C}$ at about $200 \mathrm{mbsf}$, the sediments have an age of about 16.5 $\mathrm{Ma}$ and the coring was terminated at around the middle/ lower Miocene boundary. The lowermost deposits recovered from around $221 \mathrm{mbsf}$, Hole 642B, are about $18.0 \mathrm{Ma}$ old. The mid- 


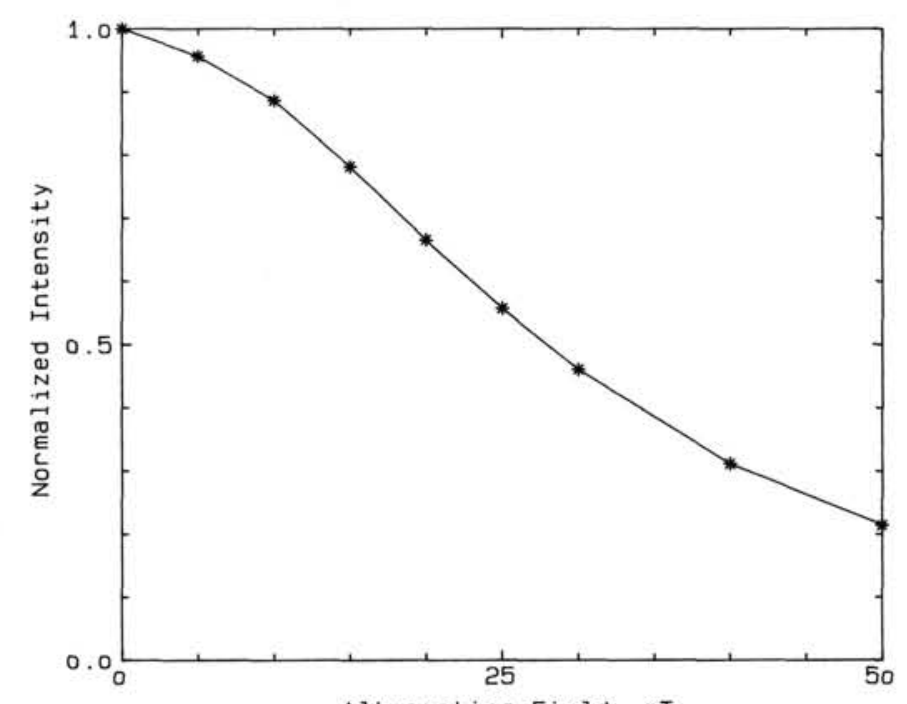

Alternating Field, mT

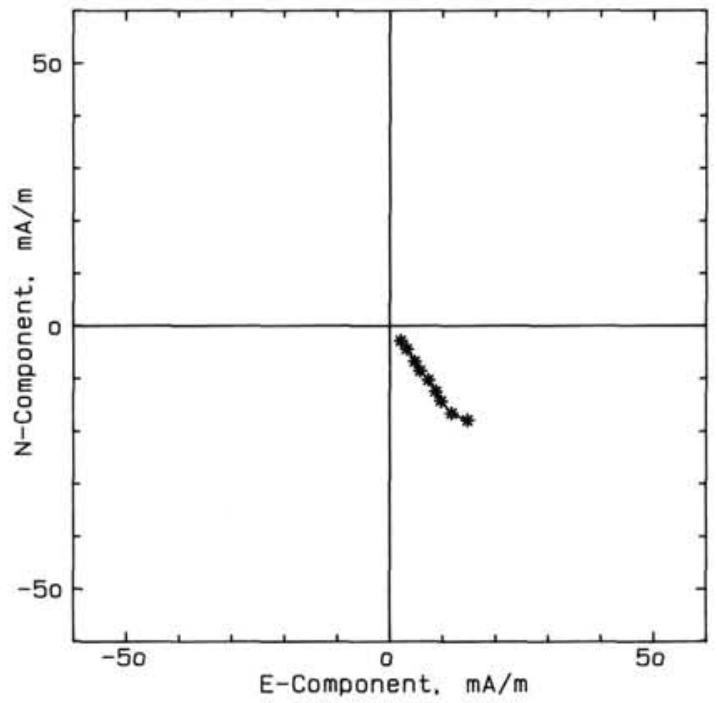

E-Component, $\mathrm{mA} / \mathrm{m}$

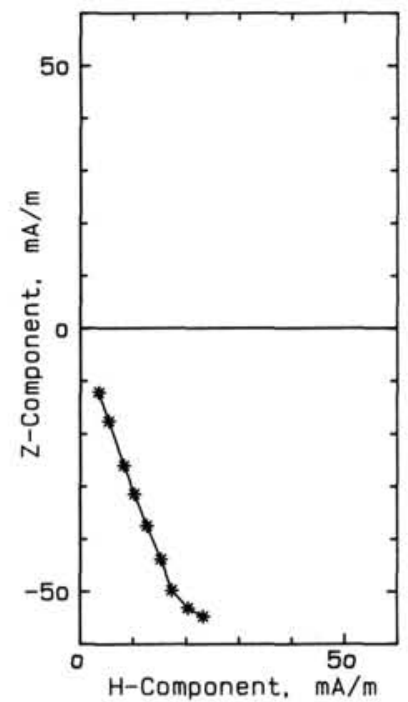

C

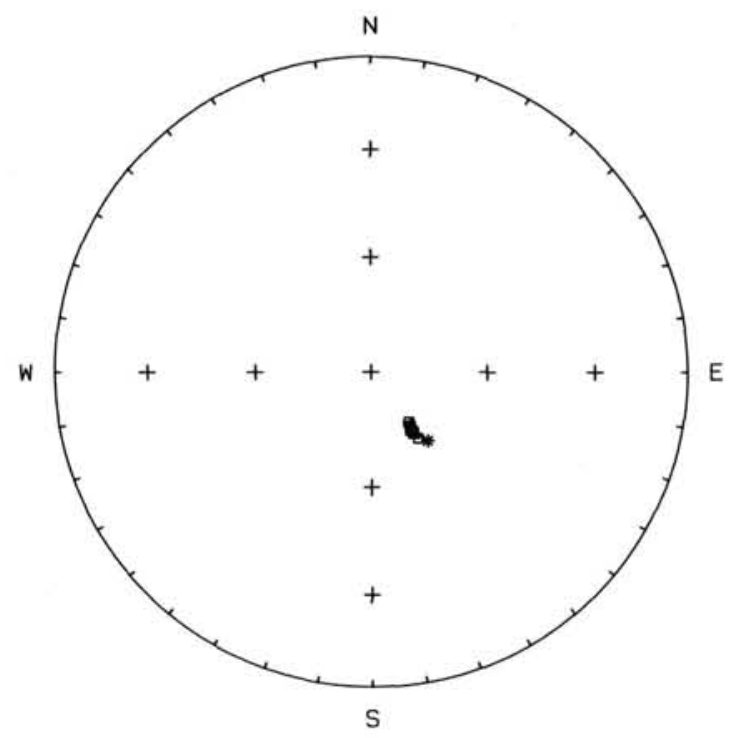

D

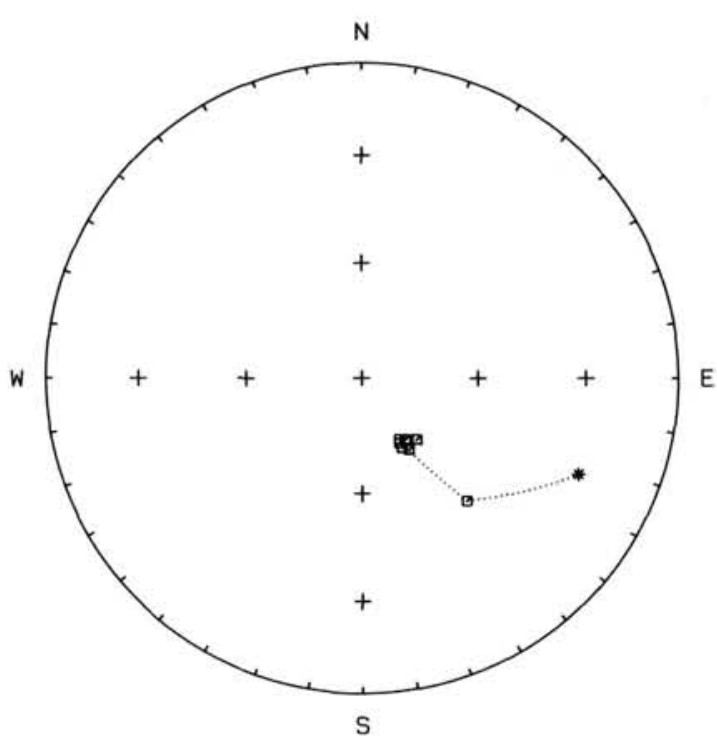

Figure 5. Demagnetization characteristics of single-component remanent magnetization (Type A). (Sample 104-642C-5H-3, 100-102 cm.) A: Demagnetization curve: Variation of the NRM-normalized remanent magnetization intensity as a function of the maximum alternating field amplitude. B: Vector diagrams: Variation of the remanent magnetization components in the horizontal plane (declination) and vertical plane (inclination). The demagnetization sequence is the same as in A. C: Stereographic projection of the resultant vectors of remanent magnetization. The asterisk indicates the NRM direction. D: Stereographic projection of the difference vectors of remanent magnetization. The asterisk indicates the first demagnetization interval. On the stereographic pro- 


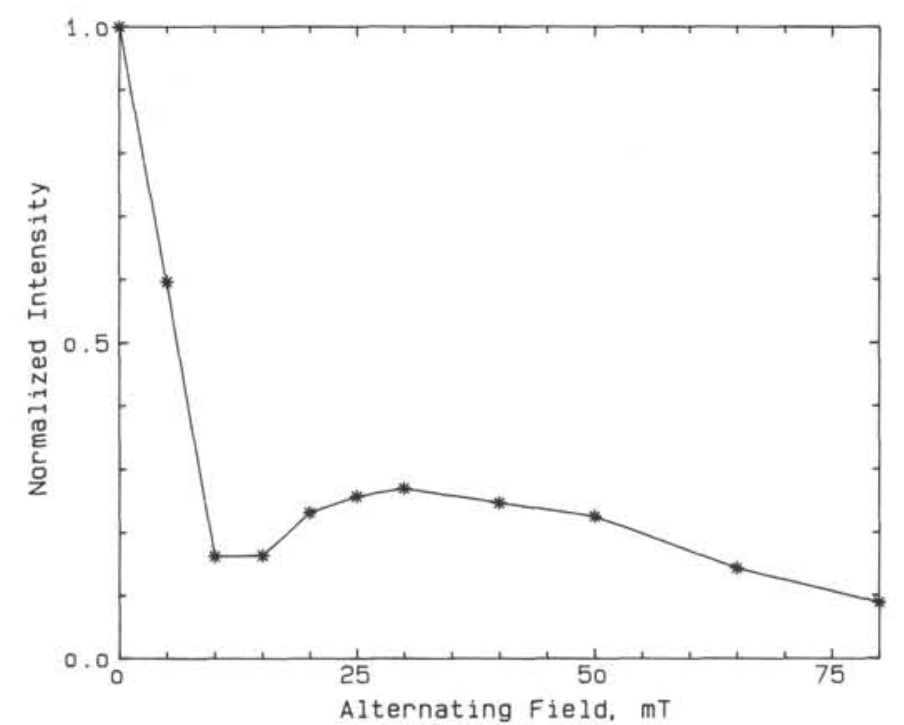

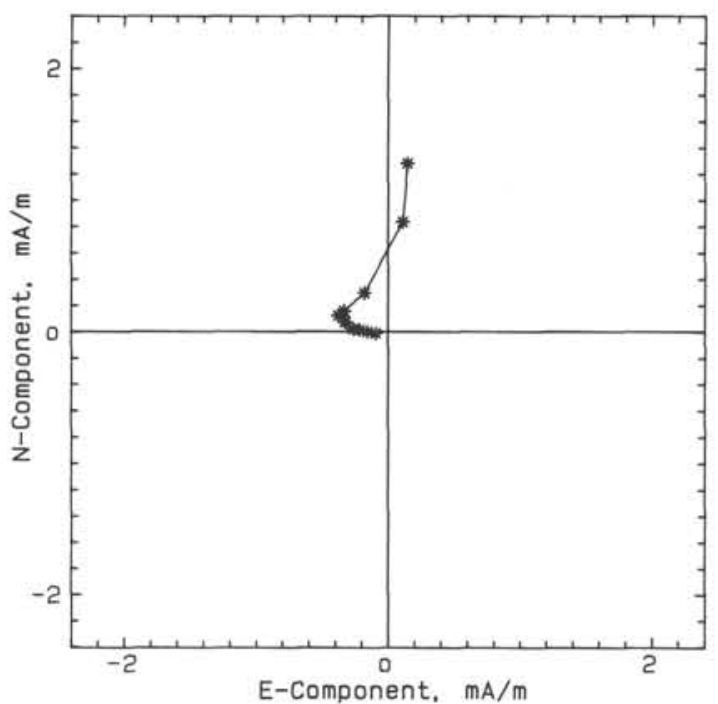

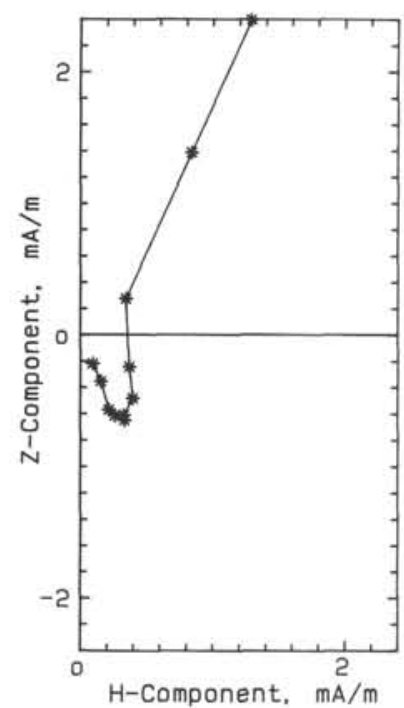

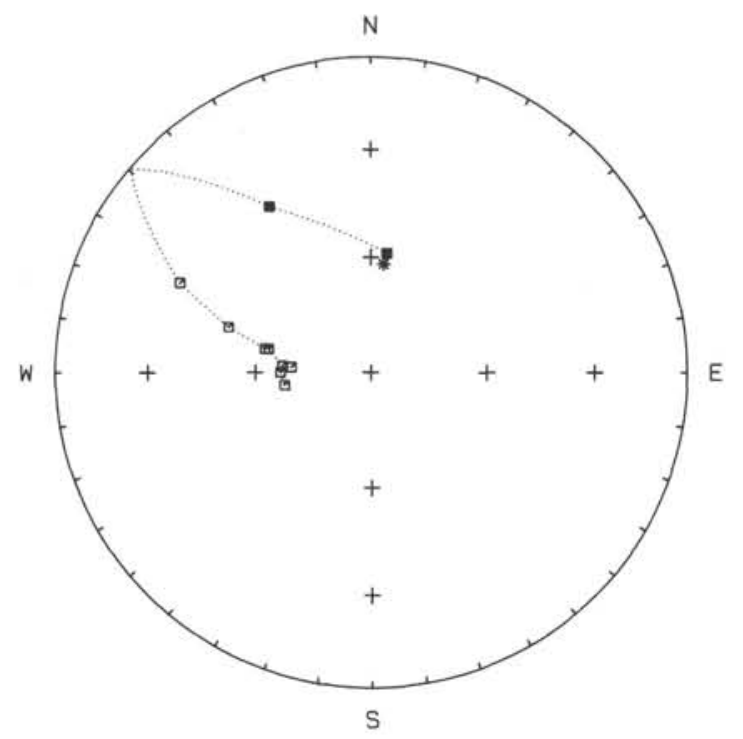

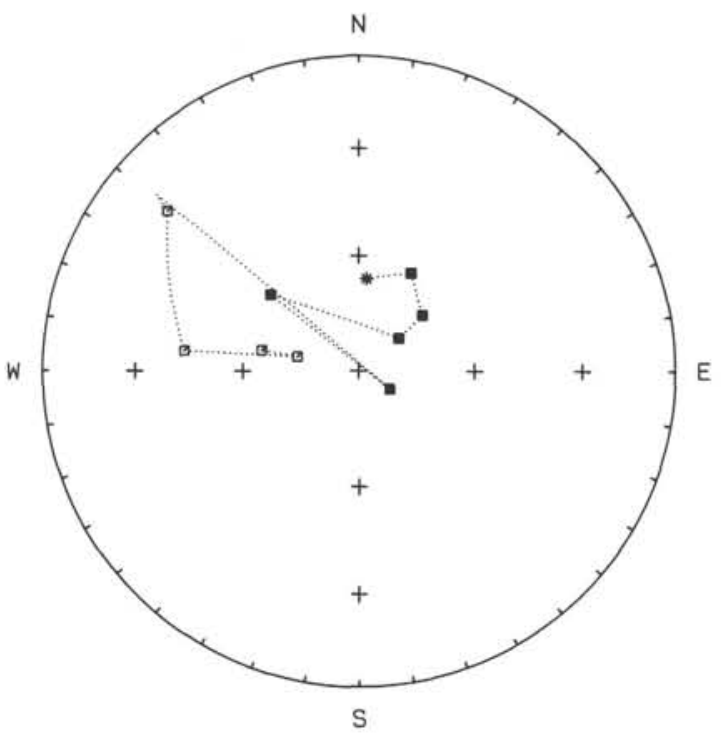

Figure 6. Demagnetization characteristics of two-component remanent magnetization (Type B). (Sample 104-643A-16H-5, 25-27 cm.) See Figure 5 caption for further explanation. 

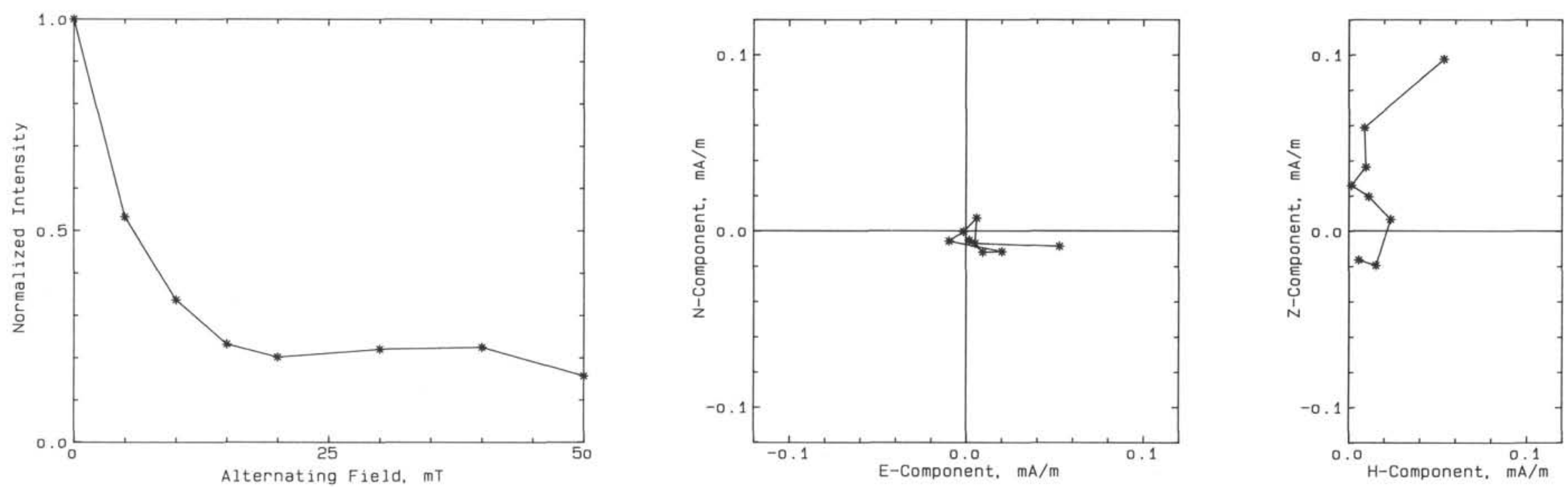

C

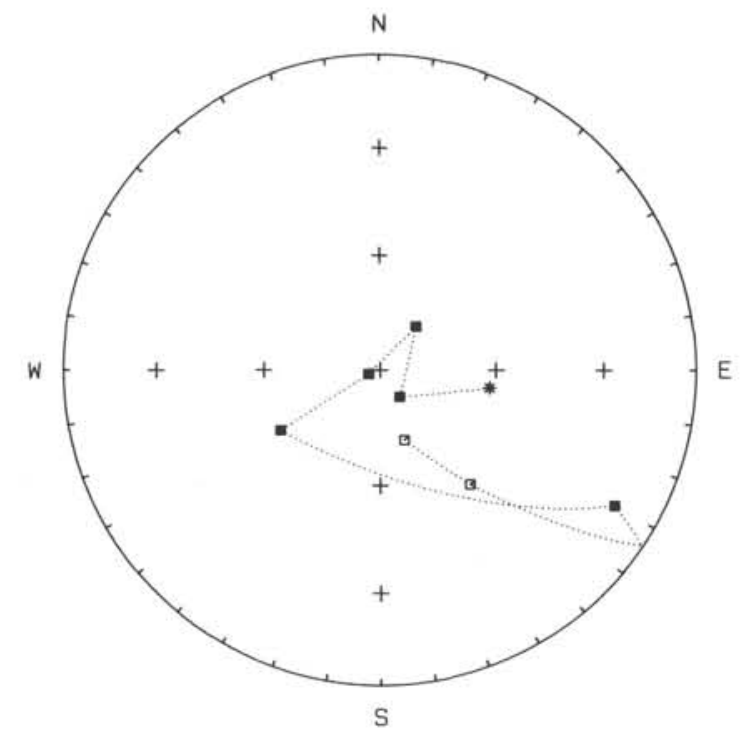

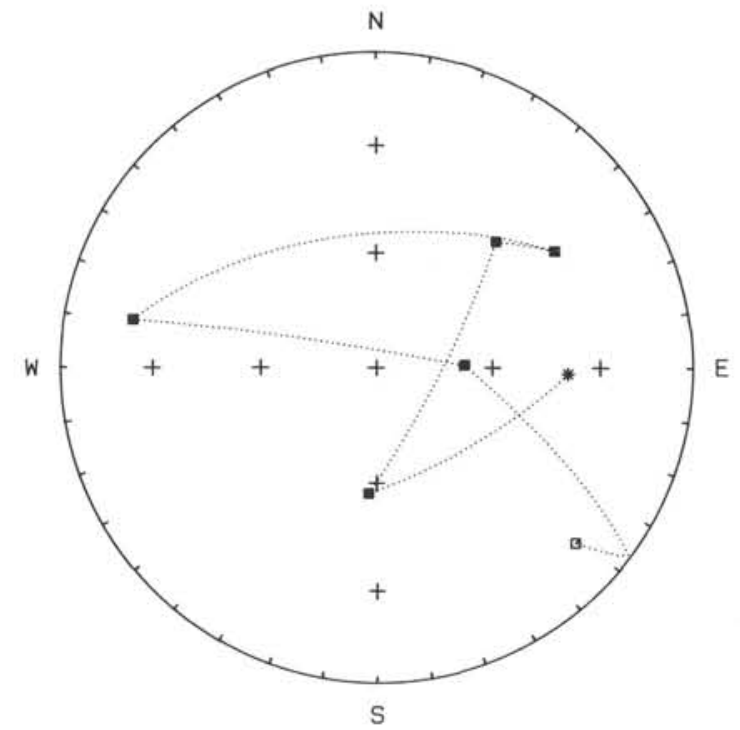

Figure 7. Demagnetization characteristics of multi-component remanent magnetization (Type C). (Sample 104-642B-10H-1, 100-102 cm.) See Figure 5 caption for further explanation. 


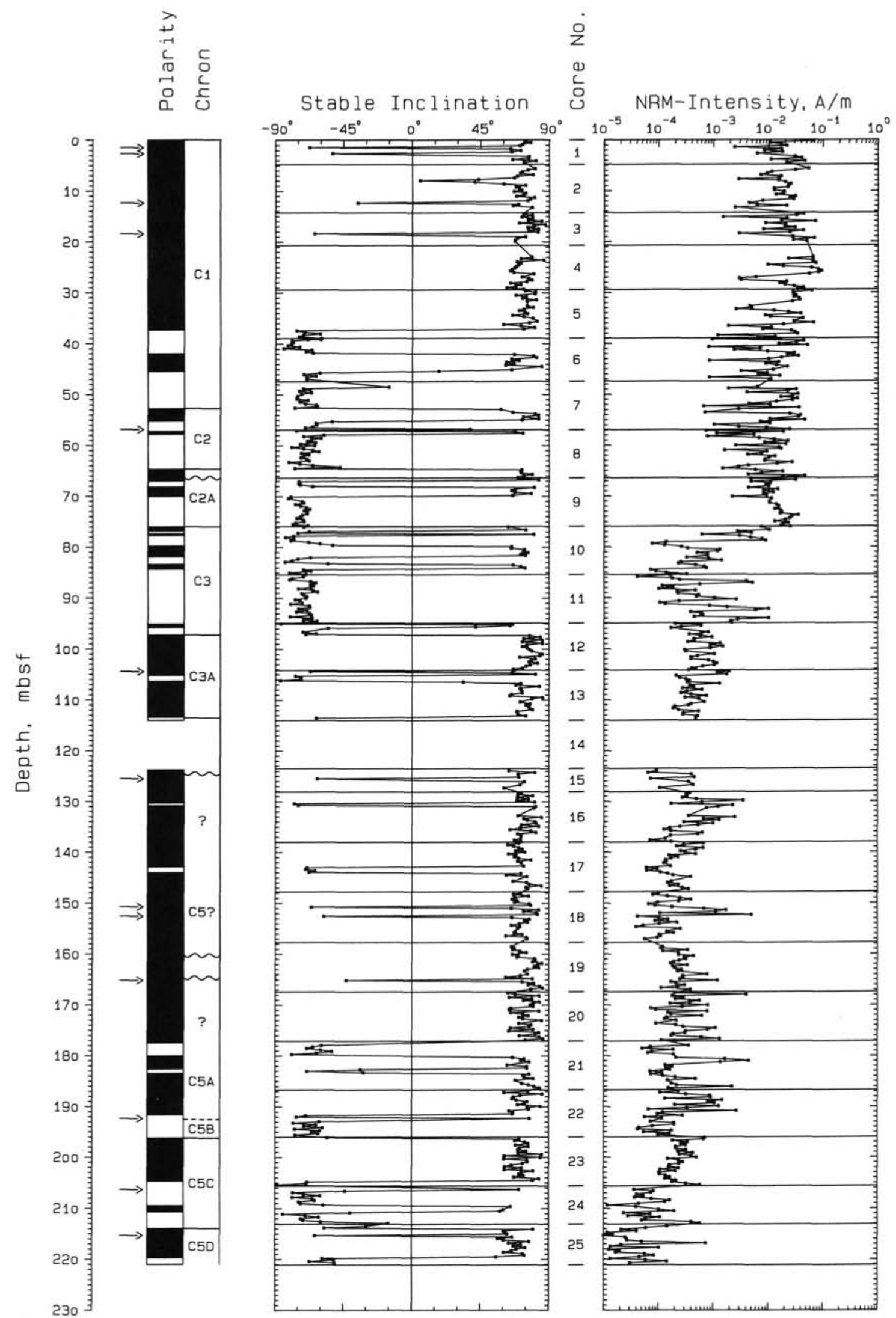

Figure 8. Paleomagnetic results for Hole 642B sedimentary series. The downhole variation in NRM intensity and stable inclination is shown together with the derived polarity log (black being normal, white reversed polarity) and the respective magnetostratigraphic interpretation in terms of the paleomagnetic chron nomenclature (cf. Fig. 2). Arrows denote apparent short polarity events documented by a single sample only. Core boundaries are marked by horizontal lines, the figures in the central column give the core numbers. 


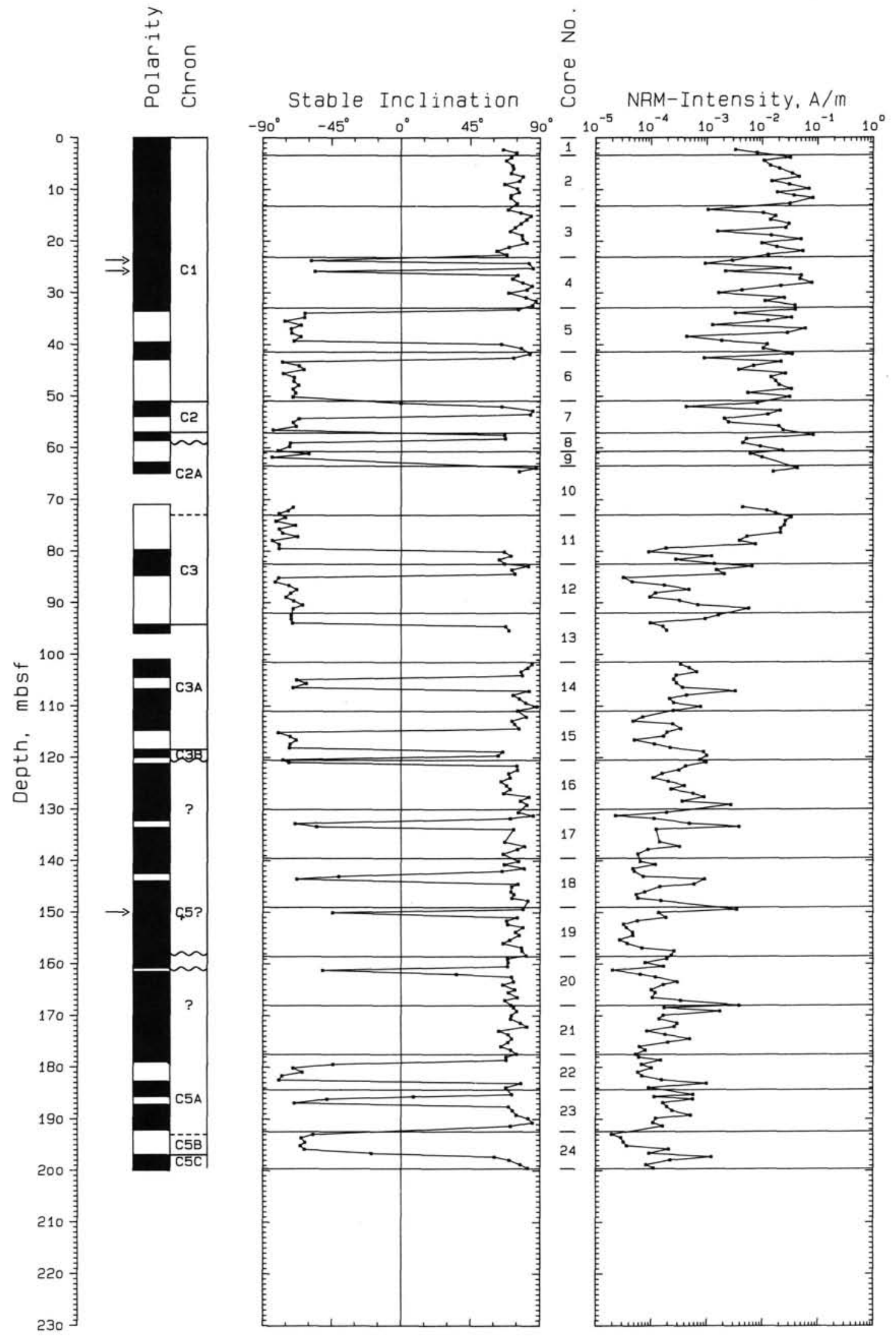

Figure 9. Paleomagnetic results for Hole $642 \mathrm{C}$ sedimentary series. See Figure 5 caption for further explanation. 
Table 2. Magnetic reversal boundaries identified in Hole 642B.

\begin{tabular}{|c|c|c|c|c|}
\hline Chron & Subchron & $\begin{array}{l}\text { Age } \\
(\mathrm{Ma})\end{array}$ & $\begin{array}{l}\text { Boundary } \\
\text { (Core, Section } \\
\text { level in } \mathrm{cm} \text { ) }\end{array}$ & $\begin{array}{l}\text { Depth } \\
\text { (mbsf) }\end{array}$ \\
\hline \multirow[t]{7}{*}{$\mathrm{Cl}$} & C1N-1 & \multirow{2}{*}{0.73} & $5-6,21$ & 37.11 \\
\hline & C1R-1 & & $5-6,50$ & 37.40 \\
\hline & CIR-1 & \multirow{2}{*}{0.91} & $6-2,141$ & 41.81 \\
\hline & $\mathrm{C} 1 \mathrm{~N}-2$ & & $6-3,21$ & 42.11 \\
\hline & $\mathrm{C} 1 \mathrm{~N}-2$ & \multirow{2}{*}{0.98} & $6-5,21$ & 45.11 \\
\hline & C1R-2 & & $6-5,81$ & 45.71 \\
\hline & C1R-2 & \multirow{2}{*}{1.66} & $7-4,69$ & 52.59 \\
\hline \multirow[t]{4}{*}{$\mathrm{C} 2$} & $\mathrm{C} 2 \mathrm{~N}$ & & $7-4,100$ & 52.90 \\
\hline & $\mathrm{C} 2 \mathrm{~N}$ & \multirow{2}{*}{1.88} & $7-6,9$ & 54.99 \\
\hline & $\mathrm{C} 2 \mathrm{R}$ & & $7-6,40$ & 55.30 \\
\hline & $\mathrm{C} 2 \mathrm{R}$ & \multirow{2}{*}{2.47} & $8-6,10$ & 64.50 \\
\hline $\mathrm{C} 2 \mathrm{~A}$ & C2AN-1 & & $8-6,40$ & 64.80 \\
\hline
\end{tabular}

$2.92 \mathrm{Ma}=\mathrm{C} 2 \mathrm{AN}-1 / \mathrm{C} 2 \mathrm{AR}-1>$ hiatus $>$ C2AR-1/

$\mathrm{C} 2 \mathrm{AN}-2=2.99 \mathrm{Ma}$

\begin{tabular}{|c|c|c|c|}
\hline $\begin{array}{l}\mathrm{C} 2 \mathrm{AN}-2 \\
\mathrm{C} 2 \mathrm{AR}-2\end{array}$ & 3.08 & $9-1,41$ & $\begin{array}{l}66.81 \\
67.11\end{array}$ \\
\hline C2AR-2 & & $9-2,11$ & 68.01 \\
\hline $\mathrm{C} 2 \mathrm{AN}-3$ & 3.18 & $9-2,41$ & 68.31 \\
\hline $\mathrm{C} 2 \mathrm{AN}-3$ & & $9-3,47$ & 69.87 \\
\hline C2AR-3 & 3.40 & $9-3,71$ & 70.11 \\
\hline C2AR-3 & & $9-7,56$ & 75.96 \\
\hline $\mathrm{C} 3 \mathrm{~N}-1$ & 3.88 & $10-1,9$ & 75.99 \\
\hline $\mathrm{C} 3 \mathrm{~N}-1$ & & $10-1,70$ & 76.60 \\
\hline C3R-1 & 3.97 & $10-1,101$ & 76.91 \\
\hline C3R-1 & & $10-1,130$ & 77.20 \\
\hline $\mathrm{C} 3 \mathrm{~N}-2$ & 4.10 & $10-2,11$ & 77.51 \\
\hline $\mathrm{C} 3 \mathrm{~N}-2$ & & $10-2,11$ & 77.51 \\
\hline C $3 \mathrm{R}-2$ & 4.24 & $10-2,41$ & 77.81 \\
\hline C3R-2 & & $10-3,71$ & 79.61 \\
\hline $\mathrm{C} 3 \mathrm{~N}-3$ & 4.40 & $10-3,100$ & 79.90 \\
\hline $\mathrm{C} 3 \mathrm{~N}-3$ & & $10-4,126$ & 81.66 \\
\hline C3R-3 & 4.47 & $10-5,11$ & 82.01 \\
\hline C $3 R-3$ & & $10-5,130$ & 83.20 \\
\hline $\mathrm{C} 3 \mathrm{~N}-4$ & 4.57 & $10-6,10$ & 83.50 \\
\hline $\mathrm{C} 3 \mathrm{~N}-4$ & & $10-6,71$ & 84.11 \\
\hline C3R-4 & 4.7 & $10-6,100$ & 84.40 \\
\hline C $3 R-4$ & & $12-2,71$ & 97.11 \\
\hline C3AN-1 & 3.3 & $12-2,101$ & 97.41 \\
\hline C3AN-1 & & $13-1,81$ & 105.01 \\
\hline C3AR-1 & 5.5 & $13-1,111$ & 105.31 \\
\hline C $3 A R-1$ & & $13-2,51$ & 106.21 \\
\hline C3AN-2 & 5.6 & $13-2,81$ & 106.51 \\
\hline C3AN-2 & 58 & $13-6,141$ & 113.11 \\
\hline C3AR-2 & 5.89 & $13-7,32$ & 113.52 \\
\hline
\end{tabular}

$6.37 \mathrm{Ma}=\mathrm{C} 3 \mathrm{AR}-2 / \mathrm{C} 3 \mathrm{BN}>2$ hiatuses; small badly disturbed recovery in Core 14; unresolved very predominantly normal polarity sequence in Cores 15 through 20 $>$ C5AR-4/C5AN-5 $=12.83 \mathrm{Ma}$

\begin{tabular}{|c|c|c|c|c|}
\hline \multirow[t]{9}{*}{ C5A } & $\begin{array}{l}\text { CSAN-5 } \\
\text { C5AR-5 }\end{array}$ & 13.01 & $\begin{array}{l}20-7,53 \\
21-1,81\end{array}$ & $\begin{array}{l}176.93 \\
177.91\end{array}$ \\
\hline & C5AR-5 & \multirow{2}{*}{13.20} & $21-2,111$ & 179.71 \\
\hline & C5AN-6 & & $21-3,21$ & 180.31 \\
\hline & C5AN-6 & \multirow{2}{*}{13.46} & $21-4,81$ & 182.41 \\
\hline & C5AR-6 & & $21-4,111$ & 182.71 \\
\hline & C5AR-6 & \multirow{2}{*}{13.69} & $21-5,21$ & 183.31 \\
\hline & C5AN-7 & & $21-5,51$ & 183.61 \\
\hline & C5AN-8 & \multirow{2}{*}{14.66} & $22-4,21$ & 191.41 \\
\hline & C5AR-8 & & $22-4,51$ & 191.71 \\
\hline C5B & C5BR-2 & \multirow{2}{*}{16.22} & $23-1,12$ & 196.12 \\
\hline C5C & $\mathrm{C} 5 \mathrm{CN}-1$ & & $23-1,41$ & 196.41 \\
\hline & $\mathrm{C} 5 \mathrm{CN}-3$ & \multirow{2}{*}{16.98} & $23-6,102$ & 204.52 \\
\hline & C5CR-3 & & $23-6,133$ & 204.83 \\
\hline \multirow{4}{*}{ C5D } & C5CR-3 & \multirow{2}{*}{17.57} & $25-1,71$ & 213.81 \\
\hline & C5DN-1 & & $25-1,101$ & 214.11 \\
\hline & C5DN-1 & \multirow{2}{*}{17.90} & $25-5,41$ & 219.51 \\
\hline & C5DR-1 & & $25-5,71$ & 219.81 \\
\hline
\end{tabular}

dle/lower Miocene boundary correlates to the C5CR-1 Subchron (Fig. 2). Its position in Hole 642B was interpolated at 199.8 mbsf as the respective short reversed polarity interval could not be identified in the paleomagnetic data (Table 2). The sediments then accumulated with an average apparent rate of $6.3 \mathrm{~m} / \mathrm{m} . \mathrm{y}$.
Table 3. Magnetic reversal boundaries identified in Hole $642 \mathrm{C}$.

\begin{tabular}{lllll}
\hline Chron & Subchron & $\begin{array}{c}\text { Age } \\
\text { (Ma) }\end{array}$ & $\begin{array}{c}\text { Boundary } \\
\text { (Core, Section } \\
\text { level in cm) }\end{array}$ & $\begin{array}{c}\text { Depth } \\
\text { (mbsf) }\end{array}$ \\
\hline C1 & C1N-1 & 0.73 & $5-1,32$ & 33.22 \\
& C1R-1 & 0.73 & $5-1,101$ & 33.91 \\
& C1R-1 & 0.91 & $5-5,33$ & 39.23 \\
& C1N-2 & & $5-5,101$ & 39.91 \\
& C1N-2 & 0.98 & $6-1,119$ & 42.59 \\
& C1R-2 & & $6-2,45$ & 43.35 \\
& C1R-2 & \multirow{2}{*}{1.66} & $6-6,119$ & 50.09 \\
C2 & C2N & & $7-1,110$ & 52.00 \\
& C2N & 1.88 & $7-2,113$ & 53.53 \\
& C2R & & $7-3,41$ & 54.31 \\
& C2R & & $7-4,113$ & 56.53 \\
C2A & C2AN-1 & 2.47 & $8-1,41$ & 57.51
\end{tabular}

$2.92 \mathrm{Ma}=\mathrm{C} 2 \mathrm{AN}-1 / \mathrm{C} 2 \mathrm{AR}-1>$ hiatus $>\mathrm{C} 2 \mathrm{AN}-2 /$

C2AR-2 $=3.08 \mathrm{Ma}$

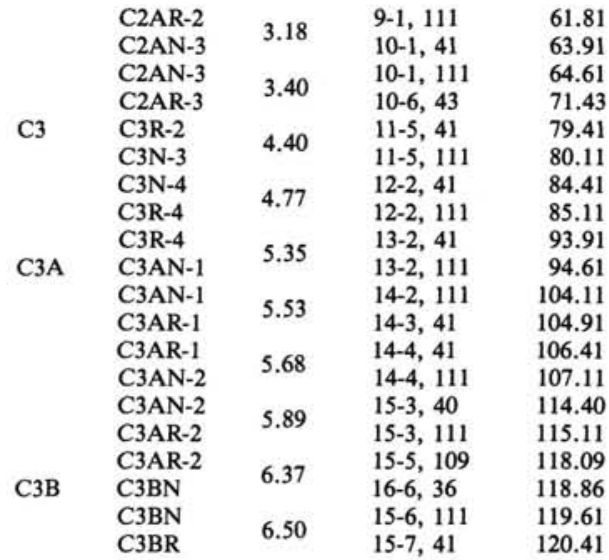

6.70 $\mathrm{Ma}=\mathrm{C} 3 \mathrm{BR} / \mathrm{C} 4 \mathrm{~N}-1>2$ hiatuses and unresolved very predominantly normal polarity sequences in Cores 16 through $21>$ C5AR-4/C5AN-5 $=12.83 \mathrm{Ma}$

\begin{tabular}{|c|c|c|c|c|}
\hline \multirow[t]{10}{*}{ C5A } & C5AN-5 & 13.01 & $22-1,110$ & $\begin{array}{l}178.61 \\
179.41\end{array}$ \\
\hline & C5AR-5 & & $22-2,41$ & $\begin{array}{l}179.41 \\
182.41\end{array}$ \\
\hline & C5AR-5 & 13.20 & $22-4,41$ & 182. \\
\hline & C5AN-6 & 15.20 & $22-4,111$ & 183 \\
\hline & C5AN-6 & & $23-1,96$ & 185. \\
\hline & C5AR-6 & 13.40 & $23-2,29$ & 186. \\
\hline & C5AR-6 & & $23-2,104$ & 186.8 \\
\hline & C5AN-7 & 15.09 & $23-3,29$ & 187.5 \\
\hline & C5AN-8 & & $23-5,104$ & 191.3 \\
\hline & C5AR-8 & 14.00 & $24-1,52$ & 192.5 \\
\hline C5B & C5BR-2 & & $24-3,118$ & 196.5 \\
\hline C5C & $\mathrm{C} 5 \mathrm{CN}-1$ & 10.22 & $24-4,38$ & 197.2 \\
\hline
\end{tabular}

during the early middle Miocene at Site 642 . Without accounting for the potential hiatus, the sedimentation rate was more than twice as high $(14.6 \mathrm{~m} / \mathrm{m}$.y.) in the short uppermost lower Miocene interval analyzed. It should be emphasized that for the magnetostratigraphic interpretation of the Holes 642B and 642C paleomagnetic records not only the biostratigraphic framework (as far as it was available for this report) was taken into account but also $\mathrm{Sr}$ isotope and $\mathrm{Rb}-\mathrm{Sr}$ glauconite ages of around $17 \mathrm{Ma}$ determined at 194.9 and $196.7 \mathrm{mbsf}$ in Hole 642B (Smalley et al., this volume).

\section{Hole $642 D$}

With Hole $642 \mathrm{D}$ the previous HPC holes were continued deeper using the extended core barrel (XCB) coring technique. Sediment drilling ended at the basalt contact encountered at 329.7 mbsf. From the top of Core 104-642D-2X at $189.9 \mathrm{mbsf}$ (Core 104-642D-1W was a washed core) to about 277 mbsf, sediments of lithologic Unit III were penetrated. This depth range 
consisted of Miocene siliceous muds and oozes. The deepest lithologic unit, Unit IV, overlying the basaltic basement, contains volcaniclastic and altered volcaniclastic muds, sandy muds, and sands of early (?) Eocene age. Paleomagnetic sampling in Hole $642 \mathrm{D}$ was limited to two samples per core section and to the interval from Core 104-642D-2X through 104-642D-15X. Below this level the recovery was too fragmentary or the sediments were too badly disturbed to be useful for paleomagnetic work. Some constraints were also imposed on the resolution of the magnetostratigraphic record in the core series analyzed by frequently incomplete or disturbed recoveries.

The magnetostratigraphies of Holes 642B and 642D are in reasonable agreement over the depth interval of overlap, although there is some mutual offset in the depth positions of identical reversal boundaries (Fig. 14). The radiolarian stratigraphy (Goll and Bjørklund, this volume) indicates three unconformities in the lower Miocene section of Hole 642D (Fig. 14). As in Hole 642B (see above), the presently preferred magnetostratigraphic interpretation assumes an interval of reduced accumulation instead of the first hiatus at 219 mbsf. The two hiatuses identified at 230 and $251 \mathrm{mbsf}$ are confined to the normal subchrons C5EN and C6N (Fig. 10, Table 4), respectively, suggesting that they comprise less than about 0.5 and $1 \mathrm{~m}$.y.

In the upper $\sim 90 \mathrm{~m}$ of sediments in Hole $642 \mathrm{D}$ for which a paleomagnetic record could be established, almost all major reversal boundaries of the geomagnetic Chrons C5B through mid C6A are recognized (Figs. 10, 14; Table 4). In this interval the apparent sedimentation rate shows only limited variability with a mean value of $14.8 \mathrm{~m} / \mathrm{m}$.y. if the potential hiatuses mentioned above are not taken into account. The deepest polarity transition that could be identified in Hole 642D (C6AN-2/C6AR-2 boundary at $277.7 \mathrm{mbsf}$ ) has an age of $21.71 \mathrm{Ma}$ and is therefore only about $2 \mathrm{~m}$.y. younger than the Miocene/Oligocene boundary (Fig. 2).

The four APC/XCB holes drilled at Site 642 document almost completely the sedimentation history of the Vøring Plateau region since the early opening of the Norwegian Sea some 57 m.y. ago. For the upper $\sim 280 \mathrm{~m}$ of the sedimentary column penetrated, paleomagnetic analyses yield a magnetostratigraphic record that spans the last $\sim 22 \mathrm{~m}$.y. and, with the exception of parts of the upper and middle Miocene, provides a high-quality stratigraphic control and resolution throughout. Unfortunately, the lowermost $\sim 50 \mathrm{~m}$ of the sediment column were found to be essentially useless for the purpose of a magnetostratigraphic study because the recovery was very low and/or showed an extensive degree of drilling disturbance.

For Pleistocene times the apparent sedimentation rate at Site 642 on average amounts to $31.8 \mathrm{~m} / \mathrm{m}$.y., decreasing from 51.0 $\mathrm{m} / \mathrm{m}$.y. during the Brunhes to $16.7 \mathrm{~m} / \mathrm{m}$.y. in the upper Matuyama Chron. During the Pliocene the average accumulation was $12.1 \mathrm{~m} / \mathrm{m} . \mathrm{y}$., increasing downhole from $9.9 \mathrm{~m} / \mathrm{m} . \mathrm{y}$. in the lower Matuyama and in the Gauss Chron to $14.0 \mathrm{~m} / \mathrm{m}$.y. during the Gilbert Chron. Note that the minor hiatus within the Gauss Chron has not been accounted for in these calculations.

Biostratigraphic analyses of the Miocene sedimentary sequences at Site 642 reveal the presence of a series of hiatuses in this epoch (Fig. 14). As discussed above, the downhole paleomagnetic polarity pattern was too fragmentary in the critical interval to quantify in detail the two potentially major unconformities in the upper and middle Miocene. According to the magnetostratigraphic record, all other hiatuses in the middle and lower Miocene span only minor periods of time and may alternatively be interpreted as periods of reduced sediment accumulation. From the Pliocene/Miocene boundary to $6.5 \mathrm{Ma}$ the average apparent sedimentation rate was $22.4 \mathrm{~m} / \mathrm{m}$.y. with a tendency to decrease with depth within this interval. For the lower part of the middle Miocene and into the lower Miocene an almost continuous magnetostratigraphic record shows that the sedimentation was rather variable. The average apparent accumulation rates for the respective periods analyzed amount to 6.3 $\mathrm{m} / \mathrm{m}$.y. in the middle Miocene-the lowest average Neogene sedimentation rate encountered in the Vøring Plateau regionand $15.0 \mathrm{~m} / \mathrm{m} . \mathrm{y}$. in the lower Miocene if the different smaller hiatuses are not accounted for.

The most remarkable feature in the natural remanent magnetization intensity logs at Site 642 is the sharp drop from some $10^{-2}$ to some $10^{-4} \mathrm{~A} / \mathrm{m}$ occurring at about $78 \mathrm{mbsf}$ (Figs. 8,9 ). This latter value is typical of that of many unconsolidated marine sediments and persists throughout lithologic Unit III. Only in the lowermost lithologic Unit IV does the NRM intensity increase again, reflecting the large volcanogenic components of these deposits. From its chronostratigraphic position in the mid Gilbert Chron, an age of about $4.3 \mathrm{Ma}$ is inferred for the Pliocene change in NRM intensity. This abrupt increase by about two orders of magnitude is believed to result from an intensified influx of terrigenous materials most likely from the adjacent Scandinavian landmass. Such a significant environmental turnover is apparently not reflected in the general lithostratigraphy, however. In particular, clear features indicative of the earliest stage of late Neogene glaciations in the Norwegian Sea (lithologic Units I/II boundary) only occur at a chronostratigraphic level of around $2.5 \mathrm{Ma}$ at Site 642.

\section{Site 643}

The most seaward site drilled during ODP Leg 104, Site 643, is located near the foot of the outer Vøring Plateau (Fig. 1). In a single hole, $643 \mathrm{~A}$, a total of $565 \mathrm{~m}$ of sediments were penetrated. The upper $50 \mathrm{~m}$ of Pliocene to Holocene glacial-interglacial sediments (lithologic Unit I) consist of alternations of dark, carbonate poor and light, carbonate-rich layers of muds and sandy muds. The underlying lithologic Unit II comprises an upper Miocene to lower Pliocene sequence of siliceous nannofossil oozes, terrigenous siliceous muds and diatomaceous nannofossil oozes. This unit is affected by slumping. Unit III, primarily diatomaceous oozes of early to middle Miocene age, extends from about 100 to $274 \mathrm{mbsf}$. Oligocene to lower Miocene dark, extremely fossil-poor terrigenous mudstones and Eocene compacted and laminated greenish to reddish zeolitic mudstones make up lithologic Units IV and V (401-565 mbsf), respectively.

Wherever possible, a paleomagnetic sampling scheme of, on average, four samples per core section was applied in Cores 104$643 \mathrm{~A}-1 \mathrm{H}$ through 104-643A-20X. Beneath, down to Core 104643A-42X, three samples were taken per section but due to a deterioration in quality and, in part, in quantity of recovery, no useful magnetostratigraphy could be established beyond about 273 mbsf (base of Core 104-643A-29X). Even down to this depth the record is of limited resolution in a number of intervals due to badly disturbed sediments or reduced recovery. Additional problems result from a series of hiatuses, some of which are rather poorly defined, both in position and extent, by the biostratigraphic data.

From the sea floor to the lower part of Core 104-643-4H the sediments contain an apparently complete standard reversal history of the last $\sim 1$ m.y. (Fig. 11). During the Brunhes Chron the average sedimentation rate was $36.4 \mathrm{~m} / \mathrm{m}$.y. Possibly because of this comparatively low rate, only one short polarity event could be resolved within the Brunhes Chron. It has an interpolated age of about $400,000 \mathrm{yr}$ and according to Jansen et al. (1988) falls within oxygen isotope stage 11. Another short polarity event was found in the younger part of the Matuyama Chron. Its position suggests that it might be the same as the one seen in the Hole 644A record (see below).

The older part of the Matuyama Chron is most likely incomplete in Hole 643A. Although no hiatus is indicated in the bio- 
U. BLEIL

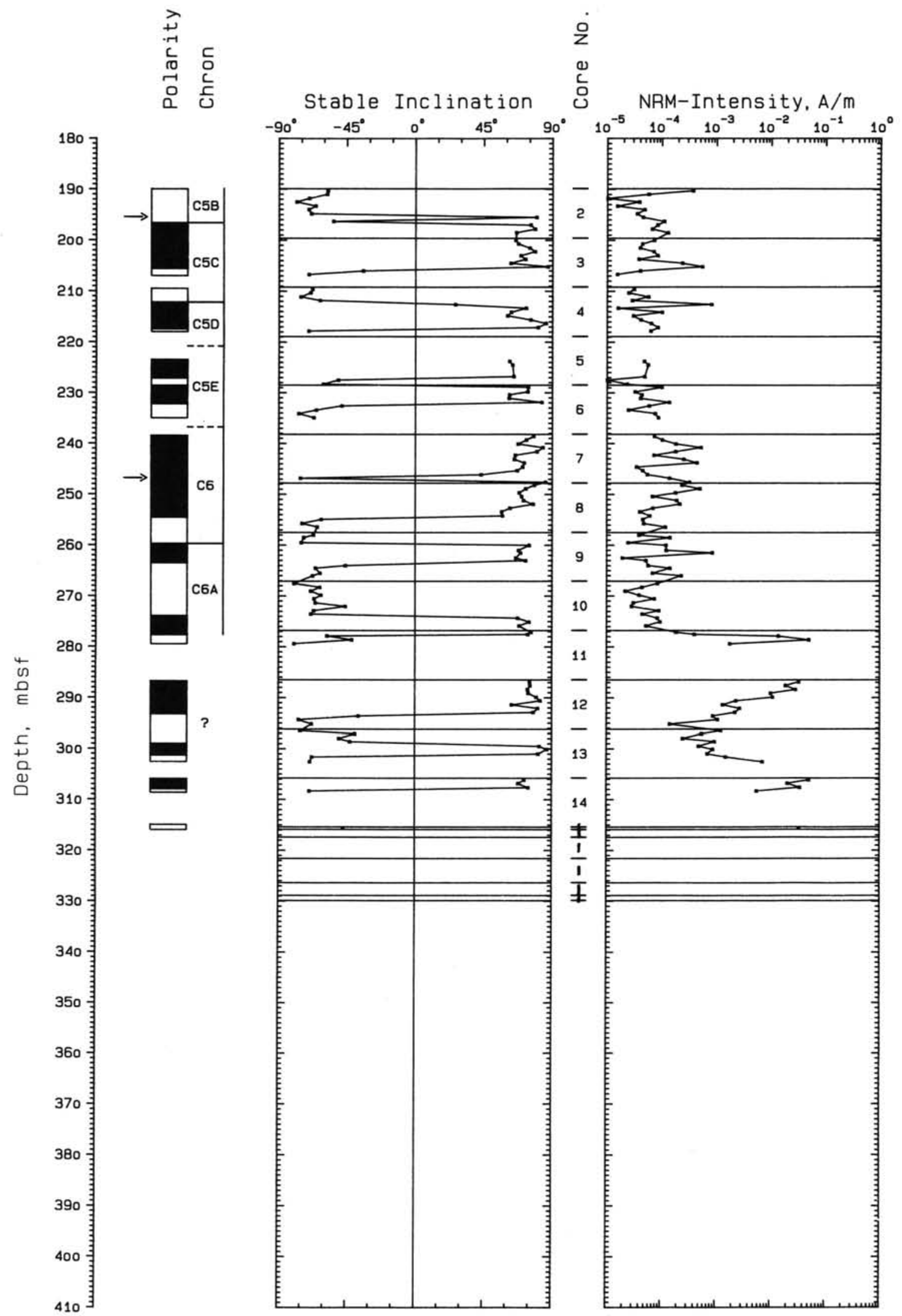

Figure 10. Paleomagnetic results for Hole 642D sedimentary series. See Figure 5 caption for further explanation. 
Table 4. Magnetic reversal boundaries identified in Hole 642D.

\begin{tabular}{|c|c|c|c|c|}
\hline Chron & Subchron & $\begin{array}{l}\text { Age } \\
\text { (Ma) }\end{array}$ & $\begin{array}{l}\text { Boundary } \\
\text { (Core, Section } \\
\text { level in } \mathrm{cm} \text { ) }\end{array}$ & $\begin{array}{l}\text { Depth } \\
\text { (mbsf) }\end{array}$ \\
\hline C5B & C5BR-2 & \multirow{2}{*}{16.22} & $2-5,41$ & 196.31 \\
\hline $\mathrm{C} 5 \mathrm{C}$ & $\mathrm{C} 5 \mathrm{CN}-1$ & & $2-5,111$ & 197.01 \\
\hline & $\mathrm{C} 5 \mathrm{CN}-3$ & \multirow{2}{*}{16.98} & $3-4,111$ & 205.21 \\
\hline & C5CR-3 & & $3-5,41$ & 206.01 \\
\hline \multirow{4}{*}{ C5D } & C5CR-3 & \multirow{2}{*}{17.57} & $4-2,111$ & 211.81 \\
\hline & C5DN-1 & & $4-3,41$ & 212.61 \\
\hline & C5DN-1 & \multirow{2}{*}{17.90} & $4-6,41$ & 217.11 \\
\hline & C5DR-1 & & $4-6,111$ & 217.81 \\
\hline \multirow[t]{3}{*}{ C5E } & CSEN & \multirow{2}{*}{19.09} & $6-3,41$ & 231.91 \\
\hline & C5ER & & $6-3,111$ & 232.61 \\
\hline & C5ER & \multirow{2}{*}{19.35} & $6-5,41$ & 234.91 \\
\hline \multirow[t]{4}{*}{ C6 } & $\mathrm{C} 6 \mathrm{~N}$ & & $7-1,41$ & 238.61 \\
\hline & $\mathrm{C} 6 \mathrm{~N}$ & \multirow{2}{*}{20.45} & $8-5,41$ & 254.21 \\
\hline & C6R & & $8-5,111$ & 254.91 \\
\hline & C6R & \multirow{2}{*}{20.88} & $9-2,51$ & 259.51 \\
\hline \multirow[t]{7}{*}{$\mathrm{C} 6 \mathrm{~A}$} & C6AN-1 & & $9-2,102$ & 260.02 \\
\hline & C6AN-1 & \multirow{2}{*}{21.16} & $9-4,102$ & 263.02 \\
\hline & C6AR-1 & & $9-5,51$ & 264.01 \\
\hline & C6AR-1 & \multirow{2}{*}{21.38} & $10-5,43$ & 273.53 \\
\hline & $\mathrm{C} 6 \mathrm{AN}-2$ & & $10-5,124$ & 274.34 \\
\hline & C6AN-2 & \multirow{2}{*}{21.71} & $11-1,76$ & 277.56 \\
\hline & C6AR-2 & & $11-1,109$ & 277.89 \\
\hline
\end{tabular}

stratigraphic data at this level, the Olduvai Subchron could not be identified, possibly because of small slumps. The normal polarity interval between 49.7 and $54.3 \mathrm{mbsf}$, the obvious candidate to be interpreted as representing the Olduvai Subchron, would imply an age of about $1.9 \mathrm{Ma}$ for the onset of glacial-interglacial cycles in the Norwegian Sea (Henrich, this volume). On the other hand, at Site 644 the respective level was found slightly older than the Matuyama/Gauss boundary in good agreement with data from the North Atlantic (Shackleton et al., 1984). To account for this discrepancy the polarity transition at 49.7 mbsf was identified as the Matuyama/Gauss boundary.

From this level to the base of Core $104-643 \mathrm{~A}-7 \mathrm{H}$ the polarity $\log$ comprises the period between geomagnetic Chrons $\mathrm{C} 2 \mathrm{~A}$ and the middle part of $\mathrm{C} 3$ (Gauss and Gilbert Chrons). The average sedimentation rate in this interval is only $6.6 \mathrm{~m} / \mathrm{m}$.y. (Fig. 14). Among other factors, in particular an unconformity within the Gauss Chron (potentially the same as seen at Site 642), this may account for some of the fine structure of the Earth's magnetic field history remaining unresolved (for details see Fig. 11 and Table 5). At the Cores $104-643 \mathrm{~A}-7 \mathrm{H} / 8 \mathrm{H}$ boundary $(62.3 \mathrm{mbsf})$ biostratigraphic analyses identified a first major hiatus at Site 643. According to the magnetostratigraphic interpretation it should span about 2 m.y. and include the Pliocene/Miocene boundary.

At the Cores $104-643 \mathrm{~A}-11 \mathrm{H} / 12 \mathrm{H}$ boundary (100.3 mbsf), in mid Core 104-643A-12 $\mathrm{H}$ (around $103.6 \mathrm{mbsf}$ ), and again in mid Core 104-643A-13H (114.2 mbsf), the radiolarian data suggest the presence of a series of three hiatuses (Goll and Bjørklund, this volume). The downhole polarity pattern in the upper Miocene interval between the first unconformity and the base of Core $104-643 \mathrm{~A}-11 \mathrm{H}$ is correlated to sections of the Chron C3A to $\mathrm{C} 4 \mathrm{~A}$ reversal sequence and yields an apparent sedimentation rate of $14.7 \mathrm{~m} / \mathrm{m} . \mathrm{y}$., the oldest reversal boundary recognized in Core 104-643A-11 H being the C4AR-2/C4AN-3 transition (8.71 $\mathrm{Ma}$ at about $97.7 \mathrm{mbsf}$ ). As a predominantly normal polarity persists in Cores 104-643A-11H through 104-643A-13H, at first sight the situation encountered seems to be more or less the same as at Site 642 . Indeed, a total of up to $6 \mathrm{~m}$.y. is represented by these unconformities around the upper/middle Miocene boundary as the magnetostratigraphic record is resumed only with the C5AN-8/C5AR-8 transition (14.66 Ma) at about $116.2 \mathrm{mbsf}$.
On the other hand, the available biostratigraphic data suggest that at least the extended normal polarities of Cores 104-643A$12 \mathrm{H}$ and $-13 \mathrm{H}$ correlate to normal subchrons in the lower part of Chron C5A and not to Subchron C5N-1 (marine magnetic anomaly 5) as at Site 642 .

Between 116.2 and about 274 mbsf where the paleomagnetic log ends, most all of the major reversal boundaries from the base of Chron C5A to the top of Chron C5E are recognized. The sediments in this entire interval accumulated at an average apparent rate of $35.8 \mathrm{~m} / \mathrm{m}$.y., which is almost the same as during Quaternary at Site 643. Downcore, the magnetostratigraphic analyses were somewhat complicated by inadequate or no recoveries in Cores 104-643A-17X, -18X, and -21X (Fig. 11). The interpretation given in Table 5 for this part of the hole may be tentative in places. The middle/lower Miocene boundary as correlated to the Subchrons C5CN-1/C5CR-1 transition is identified at $211.2 \mathrm{mbsf}$. The average sedimentation rates in the early middle Miocene and late early Miocene sections analyzed amount to 51.1 and $21.8 \mathrm{~m} / \mathrm{m} . y$., respectively. The deepest polarity reversal identified is the C5DR-2/C5EN boundary with an assigned early Miocene age of $18.56 \mathrm{Ma}$. Note that for the deeper part of the lower Miocene the sediments should have accumulated at an average rate of about $25 \mathrm{~m} / \mathrm{m}$.y. as the Miocene/Oligocene boundary is recognized at around $400 \mathrm{mbsf}$ in the biostratigraphic record.

In summary, the upper $62 \mathrm{~m}$ of sediments recovered in Hole 643A yield an essentially continuous magnetostratigraphic sequence extending from the lower Pliocene to the Holocene. Beneath, the sedimentary record is partly fragmentary. A series of unconformities originally defined from biostratigraphic analyses span a period of some $2 \mathrm{~m}$.y. around the Pliocene/Miocene boundary and another $6 \mathrm{~m}$.y. in late/middle Miocene times thus causing major gaps in information. The apparent sedimentation rate averages $33.2 \mathrm{~m} / \mathrm{m}$.y. during Pleistocene and decreases to about $6.6 \mathrm{~m} / \mathrm{m} . \mathrm{y}$. in the Pliocene, the lowest mean value encountered in the entire Neogene at Site 643. In the late Miocene on average $14.7 \mathrm{~m} / \mathrm{m}$.y. of sediments accumulated. The highest sedimentation rate, $51.1 \mathrm{~m} / \mathrm{m} . \mathrm{y}$, occurred in the middle Miocene whereas for the early Miocene a more typical value of 21.8 $\mathrm{m} / \mathrm{m} . \mathrm{y}$. is observed.

The remanent magnetization intensity log at Site 643 revealed a similar pattern as found earlier at Site 642 , namely, a drastic decrease by, on average, almost two orders of magnitude downhole (Fig. 11). However, in contrast to the previous record, at Site 643 the final shift toward low NRM intensities only occurs at a magnetostratigraphic level of around $8.0 \mathrm{Ma}(85 \mathrm{mbsf}$, Fig. 11) which is distinctly older than the respective mid Gilbert Chron horizon as at Site 642. At present there is no plausible explanation for this discrepancy. However, the fact that the change in NRM intensity is observed at about identical depth positions at both sites is a conspicuous feature and possibly the key to solve this problem.

\section{Site 644}

Of the three sites drilled during ODP Leg 104, 644 is located at the most landward position close to the inner continental slope in the Vøring Basin (Fig. 1). It has the highest sedimentation rate and the chronostratigraphic analyses were less hampered by intervals barren in useful biostratigraphic information than the other two sites. As at Site 642, a double HPC-coring strategy was employed. Some cores retrieved from deeper parts of both holes were disturbed by the high pressure of natural gas. Thus their recovery is somewhat more scattered than the average.

The upper $228.5 \mathrm{~m}$ of upper Pliocene through Holocene sediments (lithologic Unit I) are predominantly interbedded dark, carbonate-poor glacial sandy muds and light, interglacial cal- 


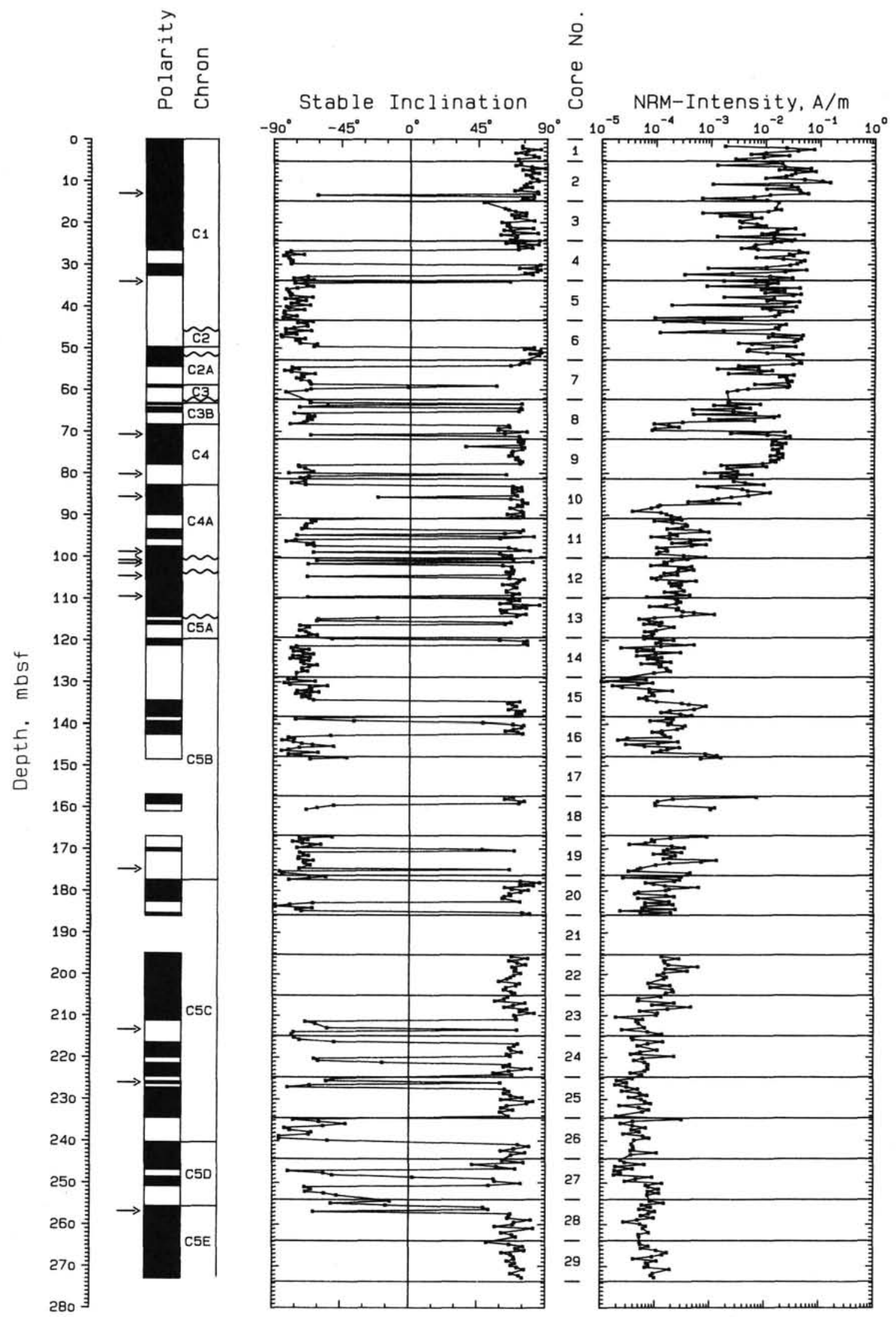

Figure 11. Paleomagnetic results for Hole 643A sedimentary series. See Figure 5 caption for further explanation. 
Table 5. Magnetic reversal boundaries identified in Hole 643A.

\begin{tabular}{|c|c|c|c|c|}
\hline Chron & Subchron & $\begin{array}{l}\text { Age } \\
\text { (Ma) }\end{array}$ & $\begin{array}{l}\text { Boundary } \\
\text { (Core, Section } \\
\text { level in cm) }\end{array}$ & $\begin{array}{l}\text { Depth } \\
(\mathrm{cm})\end{array}$ \\
\hline \multirow[t]{6}{*}{$\mathrm{Cl}$} & $\mathrm{C} 1 \mathrm{~N}-1$ & \multirow{2}{*}{0.73} & $4-2,56$ & 26.36 \\
\hline & CIR-1 & & $4-2,96$ & 26.76 \\
\hline & C1R-1 & \multirow{2}{*}{0.91} & $4-4,96$ & 29.76 \\
\hline & $\mathrm{C} 1 \mathrm{~N}-2$ & & $4-4,132$ & 30.12 \\
\hline & $\mathrm{C} 1 \mathrm{~N}-2$ & \multirow{2}{*}{0.98} & $4-6,56$ & 32.36 \\
\hline & CIR-2 & & $4-6,96$ & 32.76 \\
\hline \multicolumn{5}{|c|}{$\begin{array}{l}1.66 \mathrm{Ma}=\mathrm{C} 1 \mathrm{R}-2 / \mathrm{C} 2 \mathrm{~N}>\text { hiatus }>\mathrm{C} 2 \mathrm{~N} / \mathrm{C} 2 \mathrm{R}= \\
1.88 \mathrm{Ma}\end{array}$} \\
\hline \multirow{5}{*}{$\begin{array}{l}\mathrm{C} 2 \\
\mathrm{C} 2 \mathrm{~A}\end{array}$} & $\mathrm{C} 2 \mathrm{R}$ & \multirow[b]{2}{*}{2.47} & $6-5,26$ & 49.56 \\
\hline & $\mathrm{C} 2 \mathrm{AN}-1$ & & $6-5,56$ & 49.86 \\
\hline & C2AN-3 & \multirow{2}{*}{3.40} & $7-1,134$ & 54.14 \\
\hline & C2AR-3 & & $7-2,24$ & 54.54 \\
\hline & C2AR-3 & \multirow{2}{*}{3.88} & $7-4,129$ & 58.59 \\
\hline \multirow{3}{*}{$\mathrm{C} 3$} & $\mathrm{C} 3 \mathrm{~N}-1$ & & $7-5,25$ & 59.05 \\
\hline & $\mathrm{C} 3 \mathrm{~N}-$ & \multirow{2}{*}{3.97} & $7-5,57$ & 59.37 \\
\hline & C $3 R-1$ & & $7-5,95$ & 59.75 \\
\hline
\end{tabular}

$4.10 \mathrm{Ma}=\mathrm{C} 3 \mathrm{R}-1 / \mathrm{C} 3 \mathrm{~N}-2>$ hiatus $>\mathrm{C} 3 \mathrm{AN}-2 / \mathrm{C} 3 \mathrm{AR}-$ $2=5.89 \mathrm{Ma}$

\begin{tabular}{|c|c|c|c|c|}
\hline \multirow{3}{*}{$\begin{array}{l}\text { C3A } \\
\text { C3B }\end{array}$} & C3AR-2 & \multirow[b]{2}{*}{6.37} & $8-1,72$ & 63.02 \\
\hline & $\mathrm{C} 3 \mathrm{BN}$ & & $8-1,107$ & 63.37 \\
\hline & C3BN & \multirow{2}{*}{6.50} & $8-2,131$ & 65.11 \\
\hline & C3BR & & $8-3,25$ & 65.55 \\
\hline & C3BR & \multirow{2}{*}{6.70} & $8-4,132$ & 68.12 \\
\hline \multirow[t]{8}{*}{ C4 } & $\mathrm{C} 4 \mathrm{~N}-1$ & & $8-5,25$ & 68.55 \\
\hline & $\mathrm{C} 4 \mathrm{~N}-1$ & \multirow{2}{*}{6.78} & $8-6,57$ & 70.38 \\
\hline & C4R-1 & & $8-6,93$ & 70.73 \\
\hline & C4R-1 & \multirow{2}{*}{6.85} & $8-6,93$ & 70.73 \\
\hline & $\mathrm{C} 4 \mathrm{~N}-2$ & & $8-6,133$ & 71.13 \\
\hline & $\mathrm{C} 4 \mathrm{~N}-3$ & \multirow{2}{*}{7.41} & $9-4,136$ & 77.66 \\
\hline & C4R-3 & & $9-5,26$ & 78.06 \\
\hline & C4R-3 & \multirow{2}{*}{7.90} & $10-1,136$ & 82.66 \\
\hline \multirow[t]{8}{*}{$\mathrm{C} 4 \mathrm{~A}$} & C4AN-1 & & $10-2,26$ & 83.06 \\
\hline & C4AN-1 & \multirow{2}{*}{8.21} & $10-7,26$ & 90.56 \\
\hline & C4AR-1 & & $11-1,26$ & 91.06 \\
\hline & C4AR-1 & \multirow{2}{*}{8.41} & $11-2,96$ & 93.26 \\
\hline & C4AN-2 & & $11-2,136$ & 93.66 \\
\hline & C4AN-2 & \multirow{2}{*}{8.50} & $11-4,26$ & 95.56 \\
\hline & C4AR-2 & & $11-4,56$ & 95.86 \\
\hline & $\begin{array}{l}\text { C4AR-2 } \\
\text { C4AN-3 }\end{array}$ & 8.71 & $\begin{array}{l}11-5,56 \\
11-5,96\end{array}$ & $\begin{array}{l}97.36 \\
97.76\end{array}$ \\
\hline \multicolumn{5}{|c|}{$\begin{array}{l}8.80 \mathrm{Ma}=\mathrm{C} 4 \mathrm{AN}-3 / \mathrm{C} 4 \mathrm{AR}-3>3 \text { hiatuses }>\text { C5AR-7/ } \\
\text { C5AN-8 }=14.20 \mathrm{Ma}\end{array}$} \\
\hline \multirow[t]{3}{*}{$\mathrm{C} 5 \mathrm{~A}$} & C5AN-8 & \multirow{2}{*}{14.66} & $13-5,26$ & 116.06 \\
\hline & C5AR-8 & & $13-5,56$ & 116.36 \\
\hline & C5AR-8 & \multirow{2}{*}{14.87} & $14-1,26$ & 119.56 \\
\hline \multirow[t]{8}{*}{ C5B } & C5BN-1 & & $14-1,58$ & 119.88 \\
\hline & C5BN-1 & \multirow{2}{*}{14.96} & $14-2,26$ & 121.06 \\
\hline & C5BR-1 & & $14-2,56$ & 121.36 \\
\hline & C5BR-1 & & $15-4,96$ & 134.26 \\
\hline & C5BN-2 & 15.1 & $15-4,136$ & 134.66 \\
\hline & C5BN-2 & & $16-3,96$ & 142.26 \\
\hline & C5BR-2 & 15.2 & $16-3,136$ & 142.66 \\
\hline & C5BR-2 & & $20-1,96$ & 177.26 \\
\hline $\mathrm{C} 5 \mathrm{C}$ & $\mathrm{CsCN}-1$ & 16.22 & $20-1,136$ & 177.66 \\
\hline & $\mathrm{C} 5 \mathrm{CN}-1$ & & $23-4,136$ & 210.96 \\
\hline & C5CR-1 & 16.52 & $23-5,36$ & 211.46 \\
\hline & C5CR-1 & & $24-1,136$ & 216.26 \\
\hline & $\mathrm{C} 5 \mathrm{CN}-2$ & 16.56 & $24-2,36$ & 216.76 \\
\hline & $\mathrm{C} 5 \mathrm{CN}-2$ & & $24-7,23$ & 224.13 \\
\hline & C5CR-2 & 16.7 & $25-1,36$ & 225.06 \\
\hline & C5CR-2 & & $25-2,86$ & 227.06 \\
\hline & $\mathrm{C} 5 \mathrm{CN}-3$ & 16.8 & $25-2,136$ & 227.56 \\
\hline & $\mathrm{C} 5 \mathrm{CN}-3$ & & $25-7,36$ & 234.06 \\
\hline & C5CR-3 & 16.9 & $26-1,36$ & 234.86 \\
\hline & C5CR-3 & 17. & $26-4,86$ & 239.86 \\
\hline C5D & C5DN-1 & 17.3 & $26-5,36$ & 240.86 \\
\hline & C5DN-1 & & $27-2,86$ & 246.66 \\
\hline & CSDR-1 & 17.9 & $27-2,136$ & 247.16 \\
\hline & C5DR-1 & & $27-3,86$ & 248.16 \\
\hline & C5DN-2 & 18.1 & $27-4,30$ & 249.10 \\
\hline & C5DN-2 & & $27-5,36$ & 250.66 \\
\hline & C5DR-2 & 18. & $27-5,73$ & 251.03 \\
\hline & C5DR-2 & & $28-1,136$ & 255.46 \\
\hline C5E & CSEN & 18.56 & $28-2,36$ & 255.96 \\
\hline
\end{tabular}

careous and siliceous muds. Lithologic Unit II is composed of interbedded siliceous and mixed siliceous nannofossil oozes. In Hole $644 \mathrm{~A}$ the total length of cored section was $252.8 \mathrm{~m}$. Hole 644B penetrated $127.7 \mathrm{~m}$ of sediments.

On average, one paleomagnetic sample was taken every 50 $\mathrm{cm}$ in the sediments recovered from Holes 644A and 644B. In addition, the interval containing the Brunhes/Matuyama boundary, which had successfully been identified in the shipboard long-core cryogenic magnetometer record, was sampled in greater detail in Hole 644A (Schoenharting et al., this volume). During a later resampling, a number of horizons suspected to have recorded short reversal events within the Brunhes Chron and the reversal boundary at the base of the Jaramillo Subchron were also sampled at more closely spaced intervals in this hole.

The depth variations in stable inclination and NRM intensity together with the polarity pattern identified in the two holes are shown in Figures 12 and 13 respectively. The approximately 253 $\mathrm{m}$ of sediments penetrated in Hole 644A contain an apparently continuous and complete reversal sequence from upper Pliocene to Holocene (Table 6). In Hole 644B, which is only about $128 \mathrm{~m}$ deep, the same sequence is essentially repeated to shortly below the Jaramillo Subchron (Table 7). Down to the Brunhes/Matuyama boundary the depths of reversal boundaries in both holes appear to be identical within the limits of resolution. Below this level, with the onset of the high natural gas concentrations in the sediments, mutual offsets of several meters occur.

At the base of Hole 644A the C2AN-1/C2AR-1 boundary (top of the Kaena Subchron within the Gauss Chron) had not yet been reached and consequently the sediments there are less than $2.92 \mathrm{Ma}$. The apparent average sedimentation rate to the Matuyama/Gauss reversal boundary, observed at $225.2 \mathrm{mbsf}$, amounts to $91.2 \mathrm{~m} / \mathrm{m}$.y. It decreases from $114.3 \mathrm{~m} / \mathrm{m} . \mathrm{y}$. in the Brunhes Chron (Brunhes/Matuyama boundary at $83.12 \mathrm{mbsf}$ (Schoenharting et al., this volume) to $81.5 \mathrm{~m} / \mathrm{m}$.y. in the Matuyama Chron. At the base of Hole 644B the sediments have an extrapolated age of about $1.16 \mathrm{Ma}$.

Within the Brunhes Chron of Hole 644A a series of five short intervals was encountered for which, compared to the overall pattern, several consecutive samples gave unusually shallow or in most cases clearly negative inclinations (Fig. 12). As all these horizons were carefully checked not to exhibit particular physical disturbances, the observed magnetization directions should represent the recordings of true paleoconfigurations of the Earth's magnetic field. These short periods of field crises, whether termed reversals or excursions, typically extend over depth intervals of 0.3 to $0.5 \mathrm{~m}$ and therefore appear to have lasted no longer than about 5,000 yr at the most. With the constant sedimentation rate for the Brunhes Chron on which this assumption is based, interpolation results in absolute ages of about $90,000,130,000,200,000,240,000$, and $320,000 \mathrm{yr}$, respectively. Only the two dates at 130,000 (Blake Subchron?) and 200,000 (Biwa I Subchron?) reasonably coincide with published data (see comprehensive review by Jacobs, 1984). With respect to the various problems involved in the ODP drilling procedure and the lack of knowledge of these details of the reversal history of the Earth's field, this is perhaps not an entirely surprising result. It should be possible, however, to define more accurate ages when a complete oxygen isotope stratigraphy becomes available. In Hole 644B a similar sequence of short polarity events is apparently observed in the sediments of Brunhes age (Fig. 13) but at present they are documented only by single samples for which an accidental misorientation cannot be excluded.

The same restriction applies to the single positive inclination found at about $124 \mathrm{mbsf}$ in both holes between the Jaramillo and Olduvai Subchrons of the geomagnetic Matuyama Chron. Interpolation yields an approximate age of $1.14 \mathrm{Ma}$, almost an identical value to that reported for NW Pacific sediments (Bleil, 


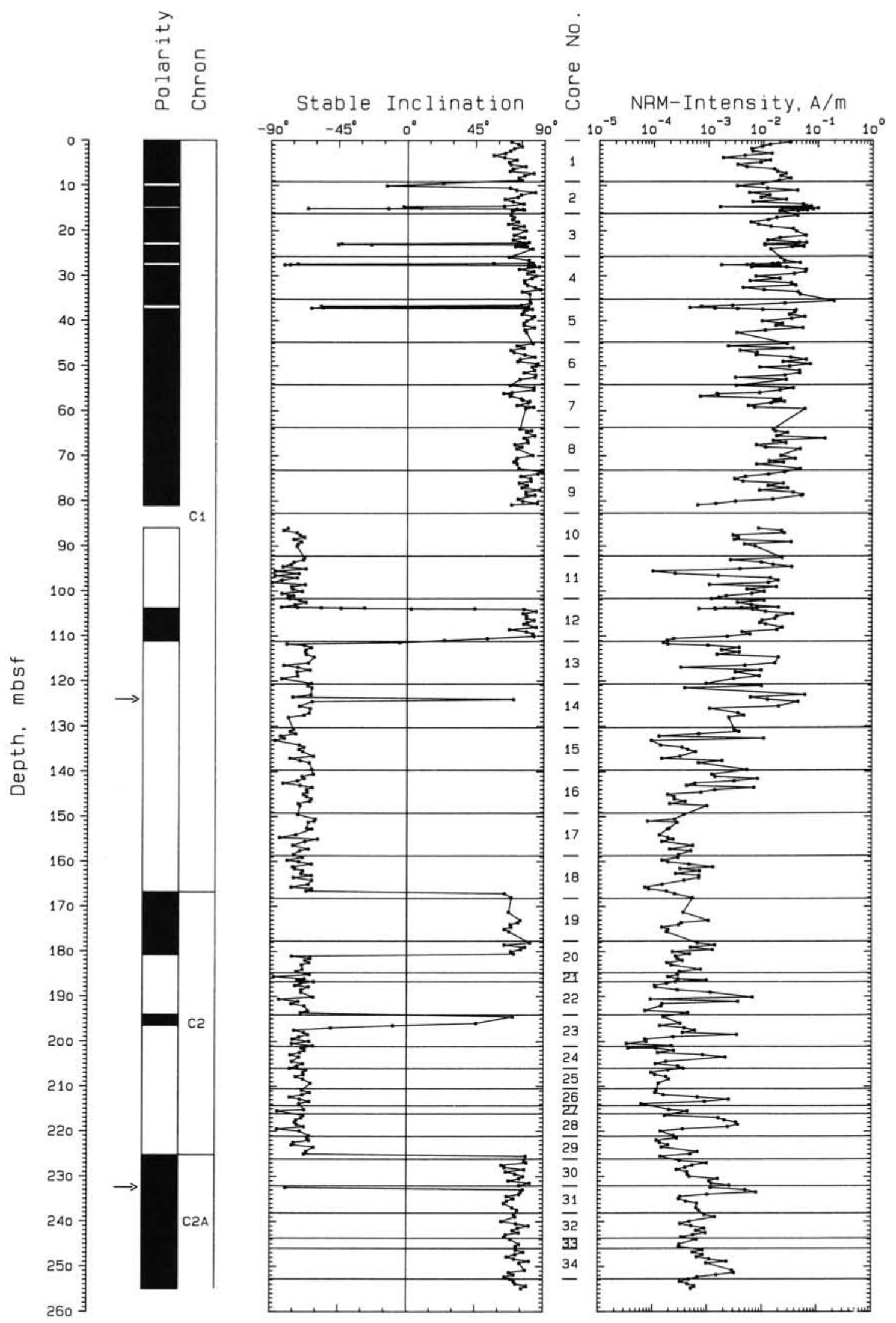

Figure 12. Paleomagnetic results for Hole 644A sedimentary series. See Figure 5 caption for further explanation. 


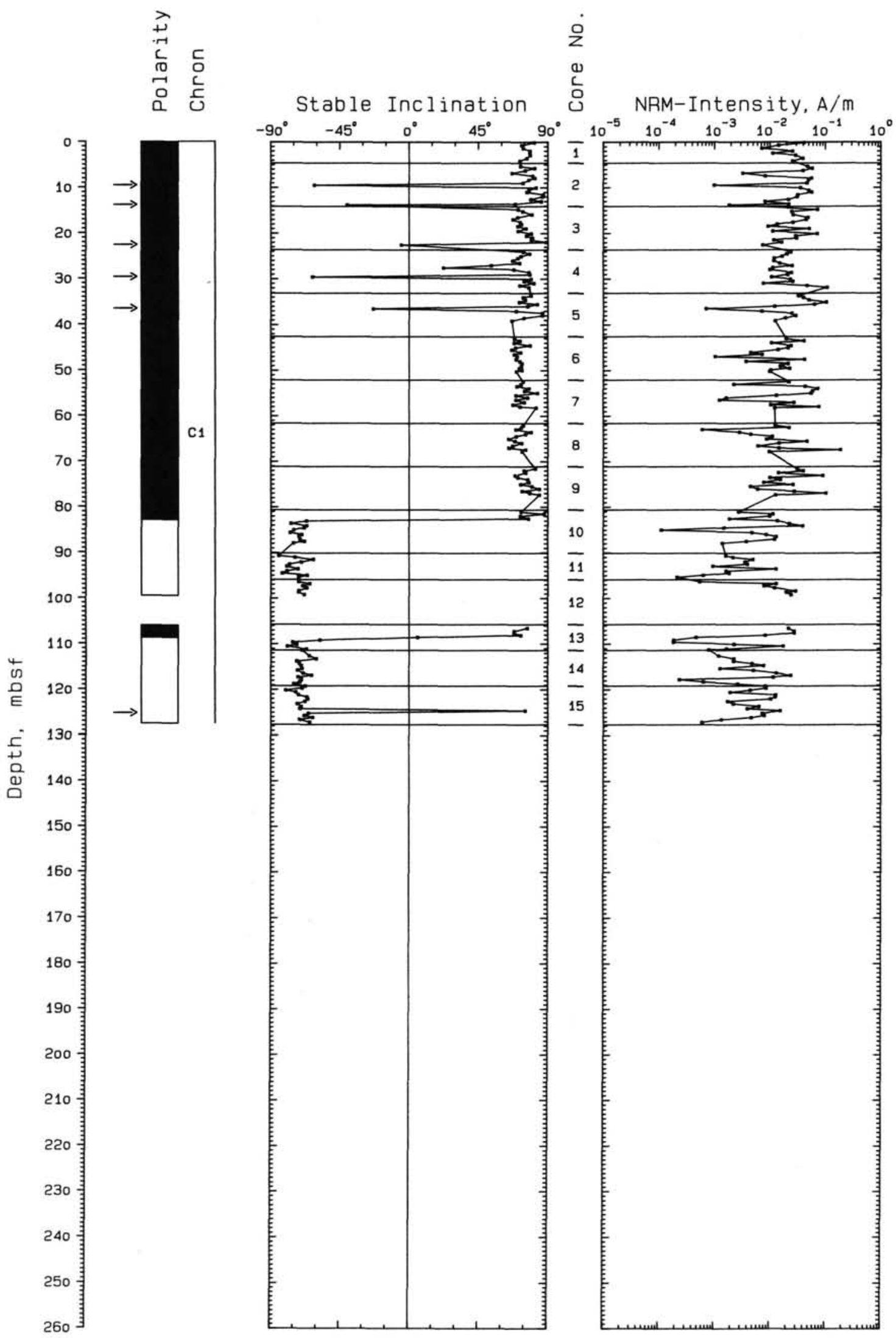

Figure 13. Paleomagnetic results for Hole 644B sedimentary series. See Figure 5 caption for further explanation. 
Table 6. Magnetic reversal boundaries identified in Hole 644A.

\begin{tabular}{|c|c|c|c|c|}
\hline Chron & Subchron & $\begin{array}{c}\text { Age } \\
\text { (Ma) }\end{array}$ & $\begin{array}{l}\text { Boundary } \\
\text { (Core, Section } \\
\text { level in } \mathrm{cm} \text { ) }\end{array}$ & $\begin{array}{l}\text { Depth } \\
\text { (mbsf) }\end{array}$ \\
\hline \multirow[t]{6}{*}{$\mathrm{Cl}$} & $\mathrm{ClN}-1$ & \multirow{2}{*}{0.73} & $9-6,14$ & $\begin{array}{l}80.84 \\
86.06\end{array}$ \\
\hline & $\begin{array}{l}\text { ClR-1 } \\
\text { C1R-1 }\end{array}$ & & $\begin{array}{l}10-3,36 \\
12-2,52\end{array}$ & $\begin{array}{r}86.06 \\
103.72\end{array}$ \\
\hline & $\begin{array}{l}\text { ClR-1 } \\
\text { CIN-2 }\end{array}$ & 0.91 & $\begin{array}{l}12-2,52 \\
12-2,86\end{array}$ & $\begin{array}{l}103.72 \\
104.06\end{array}$ \\
\hline & $\mathrm{CIN}-2$ & \multirow{2}{*}{0.98} & $12-6,136$ & 110.56 \\
\hline & C1R-2 & & $13-1,59$ & 111.79 \\
\hline & C1R-2 & \multirow{2}{*}{1.66} & $18-6,36$ & 166.56 \\
\hline \multirow[t]{4}{*}{$\mathrm{C} 2$} & $\mathrm{C} 2 \mathrm{~N}$ & & $18-6,94$ & 167.14 \\
\hline & $\mathrm{C} 2 \mathrm{~N}$ & \multirow{2}{*}{1.88} & $20-2,136$ & 180.56 \\
\hline & $\mathrm{C} 2 \mathrm{R}$ & & $20-3,36$ & 181.06 \\
\hline & $\mathrm{C} 2 \mathrm{R}$ & \multirow{2}{*}{2.47} & $29-3,86$ & 224.96 \\
\hline $\mathrm{C} 2 \mathrm{~A}$ & $\mathrm{C} 2 \mathrm{AN}-1$ & & $29-3,136$ & 225.46 \\
\hline
\end{tabular}

Table 7. Magnetic reversal boundaries identified in Hole 644B.

\begin{tabular}{|c|c|c|c|c|}
\hline Chron & Subchron & $\begin{array}{l}\text { Age } \\
\text { (Ma) }\end{array}$ & $\begin{array}{l}\text { Boundary } \\
\text { (Core, Section } \\
\text { level in } \mathrm{cm} \text { ) }\end{array}$ & $\begin{array}{l}\text { Depth } \\
\text { (mbsf) }\end{array}$ \\
\hline \multirow[t]{6}{*}{$\mathrm{Cl}$} & $\mathrm{C} 1 \mathrm{~N}-1$ & \multirow{2}{*}{0.73} & $10-2,46$ & 82.56 \\
\hline & CIR-1 & & $10-2,91$ & 83.01 \\
\hline & CIR-1 & \multirow{2}{*}{0.91} & $12-3,30$ & 99.20 \\
\hline & $\mathrm{C} 1 \mathrm{~N}-2$ & & $13-1,83$ & 106.53 \\
\hline & $\mathrm{CIN}-2$ & \multirow{2}{*}{0.98} & $13-2,91$ & 108.11 \\
\hline & CIR-2 & & $13-3,46$ & 109.16 \\
\hline
\end{tabular}

1985). Among others Clement and Robinson (1987) have identified this event several times in DSDP Leg 94 sediments from the North Atlantic. The authors also summarize the relevant literature and point out that the most probable correlation is to the Cobb Mountain Subchron (Mankinen et al., 1978), for which a radiometric age of $1.12 \mathrm{Ma}$ has been determined. In the older part of the Matuyama Chron from the Matuyama/Gauss boundary to the Olduvai Subchron a single reversal event is clearly recognized which has a mean age of around $2.07 \mathrm{Ma}$ and a duration of about $30,000 \mathrm{yr}$. This result disagrees with published polarity time scales based on radiometrically calibrated age data which note a split Réunion Subchron (McDougall, 1977) preceded by the ' $X$ ' Subchron (Mankinen \& Dalrymple, 1979). As in the NW Pacific (Bleil, 1985), the present data are more likely to indicate that only a single Réunion Subchron exists.

Finally, the result of a single sample at about $226.5 \mathrm{mbsf}$ in Hole 644A possibly also represents a short polarity event in the uppermost Gauss Chron that apparently has not yet been reported in the literature.

\section{SUMMARY AND CONCLUSIONS}

During ODP Leg 104 a total of seven sediment holes were drilled at three sites in the Vøring Plateau region of the Norwegian Sea. Using the hydraulic piston corer in the upper unconsolidated, and the extended core barrel technique in the deeper sedimentary sequences, a nearly continuous high-quality recovery was retrieved. The upper $\sim 300 \mathrm{~m}$ penetrated consist of a top layer of glacial marine deposits underlain predominantly by clays and siliceous oozes and limited intervals of calcareous oozes. To this maximum depth an extensive paleomagnetic sampling program was performed for each hole. Based on detailed alternating field demagnetization analyses, characteristic stable magnetization directions were determined for some 2500 individual samples. The magnetic reversal record thus derived for every hole could be correlated with a calibrated geomagnetic polarity time scale deduced from marine magnetic anomaly linea- tions. In particular all the Miocene sediment series analyzed contain a number of hiatuses and the magnetostratigraphies for this period could only be established in the framework of biostratigraphic data. At present, however, it is not feasible to resolve in detail all inconsistencies between the interpretation of the paleomagnetic records and the different biostratigraphic zonation schemes.

During the Pleistocene the average apparent sedimentation rate at the landward Site 644 in the Vøring Basin, $100.5 \mathrm{~m} / \mathrm{m} . \mathrm{y}$., was about three times higher than at the outer Vøring Plateau Site $642(31.8 \mathrm{~m} / \mathrm{m} . \mathrm{y}$.). At the seaward Site 643 near the foot of the Vøring Plateau, the Pleistocene/Pliocene boundary defined by the top of the geomagnetic Olduvai Subchron obviously falls into a small hiatus.

Only the uppermost part of the Pliocene was recovered in the deepest hole at Site 644. In the interval from the Pleistocene/ Pliocene boundary to the geomagnetic Matuyama-Gauss transition the sediments accumulated at an average rate of $72.0 \mathrm{~m} /$ m.y. as compared to $14.7 \mathrm{~m} / \mathrm{m}$.y. at Site 642 . For the remaining part of the Pliocene the sedimentation rates amount to 11.3 and $6.6 \mathrm{~m} / \mathrm{m} . \mathrm{y}$. at Sites 642 and 643 , respectively.

During the late late Miocene the apparent sedimentation rate was $22.4 \mathrm{~m} / \mathrm{m}$.y. at Site 642 . This sequence ends at a major hiatus. The following $\sim 40 \mathrm{~m}$ of sediments did not yield any useful paleomagnetic record. For the older upper Miocene and also the younger middle Miocene sedimentary series, therefore, there is no magnetostratigraphic age control available at this site. At Site 643 , only the middle part of the upper Miocene is documented by sediments which accumulated at an apparent rate of $14.7 \mathrm{~m} / \mathrm{m}$.y. Both the Pliocene/Miocene and the upper/middle Miocene boundaries fall within unconformities. The younger spans about $2 \mathrm{~m}$.y.; the older, which is composed of a series of three hiatuses, spans a total of $6 \mathrm{~m}$.y. It is underlain by early middle Miocene deposits.

In the early middle Miocene the sediments at Site 642 accumulated at the remarkably low average rate of $6.3 \mathrm{~m} / \mathrm{m}$.y. whereas during the same period the by far highest Miocene sedimentation rate in the Vøring Plateau region is observed at Site $643(51.1 \mathrm{~m} / \mathrm{m} . \mathrm{y}$.$) .$

At Site 642 a lower Miocene magnetostratigraphy could be established to about $2 \mathrm{~m}$.y. above the Miocene/Oligocene boundary. The lower Miocene sediments accumulated at a comparatively constant average rate of $14.6 \mathrm{~m} / \mathrm{m}$.y. if a potentially small hiatus is not taken into account. At Site 643 the magnetostratigraphic record ends at a level with an assigned lower Miocene sediment age of about $18.6 \mathrm{Ma}$. To this horizon the average apparent sedimentation rate amounts to $21.8 \mathrm{~m} / \mathrm{m}$.y.

Despite former DSDP drilling operations in the area, the combined results of the three ODP Leg 104 drill sites represent the first relatively continuous high-quality magnetostratigraphic framework from near the Miocene/Oligocene boundary to the Holocene ever obtained in the Norwegian Sea. It therefore should be extremely useful in further developing understanding of the paleoceanographic evolution of this region, in particular since the onset of the late Cenozoic glaciation cycles. Furthermore, the present set of magnetostratigraphic data constitutes a detailed basis to elaborate the yet fragmentary biochronologies in northern high latitudes, together with recent chronostratigraphic results of DSDP Legs 81 (Backman et al., 1985) and 94 (Baldauf et al., 1987) from the North Atlantic.

\section{ACKNOWLEDGMENTS}

I thank the many people responsible for the success of ODP Leg 104 , in particular the scientific party for their friendly cooperation and voluntary assistance in an extensive shipboard sampling program. Norbert R. Nowaczyk and Maria Tegeler helped with the laboratory work, the figures were drafted by Volkhard Spiess. Appreciation is also due to 


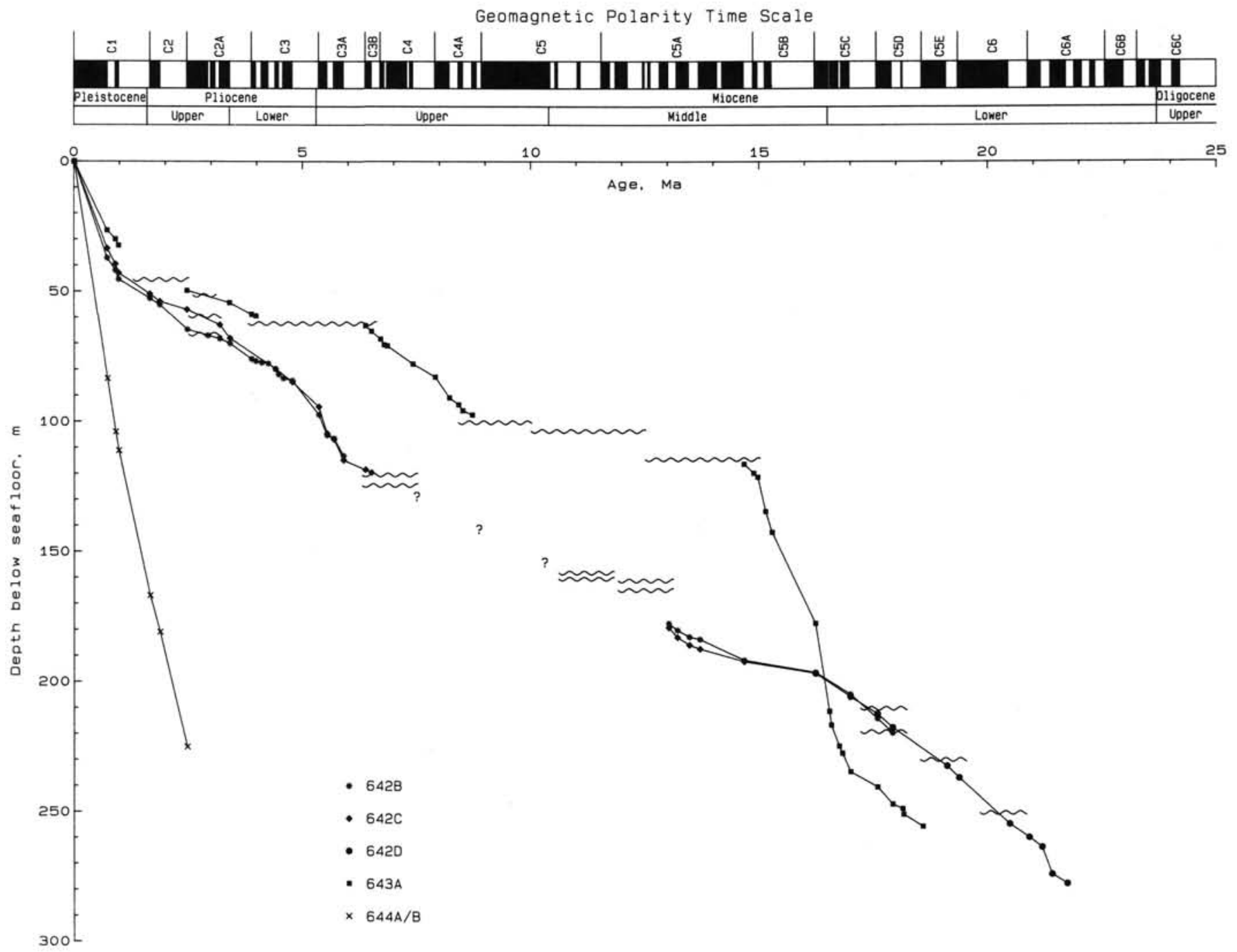

Figure 14. Sedimentation rate summary based on paleomagnetic data. The downhole pattern in remanent magnetization polarity at each site is correlated with the polarity time scale of Berggren et al. (1987). Depth positions of the hiatuses indicated are exclusively defined by biostratigraphic results.

Drs. Reidar Løvlie and Ernest A. Hailwood for critically reviewing the manuscript.

The financial support of the Deutsche Forschungsgemeinschaft both toward the Ocean Drilling Program and the paleomagnetic laboratory in Bremen is gratefully acknowledged.

\section{REFERENCES}

Backman, J., Westberg-Smith, M. J., Baldauf, J. G., Brown, S., Bukry, D., Edwards, L., Harland, R., and Huddlestun, P., 1985. Biostratigraphy of Leg 81 sediments-a high latitude record. In Roberts, D. G., Schnitker, D., et al., Init. Repts. DSDP, 81: Washington (U.S. Govt. Printing Office), 855-860.

Baldauf, J. G., Thomas, E., Clement, B., Takayama, T., Weaver, P.P.E., Backman, J., Jenkins, G., Mudie, P. J., and Westberg-Smith, M. J., 1987. Magnetostratigraphic and biostratigraphic synthesis, Deep Sea Drilling Project Leg 94. In Ruddiman, W. F., Kidd, R. B., Thomas, E., et al., Init. Repts. DSDP, 94: Washington (U.S. Govt. Printing Office), 1159-1205.

Berggren, W. A., Kent, D. V., and Van Couvering, J. A., 1987. Neogene geochronology and chronostratigraphy. In Snelling, N.J. (Ed.), The Chronology of the Geological Record. Geol. Soc. London Mem., 10:211-250.

Bleil, U., 1985. The magnetostratigraphy of Northwest Pacific sediments, Deep Sea Drilling Project Leg 86. In Heath, G. R., Burckle,
L. H., et al., Init. Repts. DSDP, 86: Washington (U.S. Govt. Printing Office), 441-458.

Clement, B. M., and Robinson, F., 1987. The magnetostratigraphy of Leg 94 sediments. In Ruddiman, W. F., Kidd, R. B., Thomas, E., et al., Init. Repts. DSDP, 94: Washington (U.S. Govt. Printing Office), 635-650.

Eldholm, O., Thiede, J., Taylor, E., et al., 1987. Proc. ODP, Init. Repts., 104: College Station, TX (Ocean Drilling Program).

Jacobs, J. A., 1984. Reversals of the Earth's Magnetic Field: Bristol (Adam Hilger Ltd.).

Jansen, E., Bleil, U., Henrich, R., Kringstad, L., and Slettemark, B., 1988. Paleoenvironmental changes in the Norwegian Sea and the Northeast Atlantic during the last 2.8 Ma.: DSDP/ODP Sites 610, 642, 643 and 644. Paleoceanography, 3:563-587.

LaBrecque, J. L., Hsü, K. J., Carman, M. F., Jr., Karpoff, A., McKenzie, J. A., Percifal, S. F., Jr., Petersen, N. P., Pisciotto, K. A., Schreiber, E., Tauxe, L., Tucker, P., Weissert, H. J., and Wright, R., 1983. DSDP Leg 73: Contributions to Paleogene stratigraphy in nomenclature, chronology and sedimentation rates. Palaeogeogr., $\mathrm{Pa}$ laeoclimatol., Palaeoecol., 42:91-125.

LaBrecque, J. L., Kent, D. V., and Cande, S. C., 1977. Revised magnetic polarity time scale for Late Cretaceous and Cenozoic time. Geology, 5:330-335.

Mankinen, E. A., and Dalrymple G. B., 1979. Revised geomagnetic polarity time scale for the interval 0-5 m.y.B.P. J. Geophys. Res., 84: 615-626. 
Mankinen, E. A., Donnilly-Nolan, J. E., Grommé, C. S., 1978. Geomagnetic polarity event recorded at 1.1 m.y.B.P. on Cobb Mountain, Clear Lake Volcanic Field, California. Geology, 6:653-656.

McDougall, I., 1977. The present status of the geomagnetic polarity time scale. Research School of Earth Sciences, Australian National University Publ. 1288.

McDougall, I., Watkins, N. D., Walker, G., and Kristjansson, L., 1976. $\mathrm{K}-\mathrm{Ar}$ and paleomagnetic analysis of Icelandic lava flows: limits on the age of Anomaly 5. J. Geophys. Res., 81:1505-1512.
Shackleton, N.J., Backman, J., Zimmerman, H., Kent, D.V., Hall, M.A., et al., 1984. Oxygen isotope calibration of the onset of icerafting in DSDP 522A: History of glaciation in the North Atlantic Region. Nature, 307:620-623.

Date of initial receipt: 5 October 1987

Date of acceptance: 12 May 1988

Ms 104B-181 
APPENDIX

TABLES OF PALEOMAGNETIC DATA

Table A1. Paleomagnetic properties of samples from Hole 642B.

\begin{tabular}{|c|c|c|c|c|c|c|c|c|}
\hline $\begin{array}{l}\text { Core, Section, } \\
\text { Interval }(\mathrm{cm})\end{array}$ & $\begin{array}{l}\text { Depth } \\
\text { (mbsf) }\end{array}$ & $\begin{array}{c}\mathrm{J} \\
\mathrm{NRM} \\
(\mathrm{mA} / \mathrm{m})\end{array}$ & $\begin{array}{l}\text { Incl. } \\
\text { NRM } \\
\text { (deg) }\end{array}$ & $\begin{array}{l}\text { Decl. } \\
\text { NRM } \\
\text { (deg) }\end{array}$ & $\begin{array}{c}\text { Incl } \\
\text { stable } \\
\text { (deg) }\end{array}$ & $\begin{array}{l}\text { Decl. } \\
\text { stable } \\
\text { (deg) }\end{array}$ & Pol. & $\begin{array}{l}\text { MDF } \\
(\mathrm{mT})\end{array}$ \\
\hline $1,1,19-21$ & .20 & 64.25 & +74.5 & 145.5 & +73.7 & 151.2 & $\mathrm{~N}$ & 32.3 \\
\hline $1,1,49-51$ & .50 & 10.49 & +70.9 & 68.4 & +77.9 & 135.4 & $\mathrm{~N}$ & 17.0 \\
\hline $1,1,79-81$ & .80 & 16.82 & +78.7 & 148.0 & +72.5 & $148 . ?$ & $\mathrm{~N}$ & 23.3 \\
\hline $1,1,109-111$ & 1.10 & 21.40 & +77.7 & 104.1 & +70.7 & 132.8 & $\mathrm{~N}$ & 17.1 \\
\hline $1,1,139-141$ & 1.40 & 2.38 & -24.6 & 327.6 & -67.7 & 319.3 & & 59.5 \\
\hline $1,2,19-21$ & 1.70 & 17.08 & +50.7 & 125.1 & +64.7 & 128.4 & $\mathrm{~N}$ & 18.1 \\
\hline $1,2,49-51$ & 2.00 & 8.25 & +62.5 & 115.5 & +71.3 & 139.9 & $\mathrm{~N}$ & 11.6 \\
\hline $1,2,79-81$ & 2.30 & 17.82 & +61.4 & 154.7 & +65.4 & 148.0 & $\mathrm{~N}$ & 19.9 \\
\hline $1,2,109-111$ & 2.60 & 6.18 & +2.1 & 120.0 & -52.5 & 156.7 & & 27.7 \\
\hline $1,3,19-21$ & 3.20 & 30.32 & +74.3 & 146.7 & +76.7 & 134.8 & $\mathrm{~N}$ & 26.6 \\
\hline $1,3,49-51$ & 3.50 & 40.50 & +74.2 & 157.4 & +76.4 & 138.3 & $\mathrm{~N}$ & 22.5 \\
\hline $1,3,79-81$ & 3.80 & 11.01 & +70.1 & 84.5 & +65.8 & 142.0 & $\mathrm{~N}$ & 10.6 \\
\hline $1,3,109-111$ & 4.10 & 46.29 & +81.2 & 141.0 & +81.3 & 150.4 & $\mathrm{~N}$ & 25.5 \\
\hline $1,3,141-143$ & 4.42 & 21.30 & +70.9 & 131.2 & +73.4 & 144.4 & $\mathrm{~N}$ & 15.5 \\
\hline $2,1,80-82$ & 5.61 & 54.17 & +83.3 & 135.3 & +80.6 & 120.4 & $\mathrm{~N}$ & 28.1 \\
\hline $2,1,109-111$ & 5.90 & 30.91 & +74.1 & 76.1 & +75.9 & 114.6 & $\mathrm{~N}$ & 18.0 \\
\hline $2,1,139-141$ & 6.20 & 11.31 & +82.5 & 192.9 & +72.3 & 120.2 & $\mathrm{~N}$ & 4.2 \\
\hline $2,2,20-22$ & 6.51 & 9.34 & +87.8 & 225.5 & +71.0 & 105.6 & $\mathrm{~N}$ & 9.7 \\
\hline $2,2,50-52$ & 6.81 & 7.15 & +87.3 & 308.0 & +79.4 & 133.6 & $\mathrm{~N}$ & 4.8 \\
\hline $2,2,80-82$ & 7.11 & 16.90 & +63.1 & 117.4 & +68.8 & 114.8 & $\mathrm{~N}$ & 22.0 \\
\hline $2,2,110-112$ & 7.41 & 15.59 & +51.1 & 107.1 & +66.9 & 103.9 & $\mathrm{~N}$ & 13.2 \\
\hline $2,2,140-142$ & 7.71 & 2.81 & +34.2 & 155.3 & +43.3 & 187.4 & & 16.1 \\
\hline $2,3,10-12$ & 7.91 & 15.07 & +18.1 & 302.5 & +5.0 & 263.9 & & 9.7 \\
\hline $2,3,44-46$ & 8.25 & 20.03 & +47.1 & 290.7 & +41.1 & 276.1 & & 17.7 \\
\hline $2,3,79-81$ & 8.60 & 25.51 & +59.6 & 339.5 & +60.2 & 44.3 & $\mathrm{~N}$ & 22.0 \\
\hline $2,3,110-112$ & 8.91 & 22.94 & +63.0 & 14.9 & +74.4 & 46.6 & $\mathrm{~N}$ & 12.1 \\
\hline $2,3,140-142$ & 9.21 & 22.30 & +69.2 & 52.8 & +68.1 & 48.1 & $\mathrm{~N}$ & 16.5 \\
\hline $2,4,19-21$ & 9.50 & 12.38 & +70.2 & 45.8 & +70.3 & 36.2 & $\mathrm{~N}$ & 19.2 \\
\hline $2,4,50-52$ & 9.81 & 12.52 & +72.7 & 132.1 & +71.9 & 136.2 & $\mathrm{~N}$ & 22.2 \\
\hline $2,4,80-82$ & 10.11 & 19.19 & +70.2 & 124.5 & +66.7 & 136.4 & $\mathrm{~N}$ & 18.4 \\
\hline $2,4,110-112$ & 10.41 & 18.70 & +79.4 & 138.2 & +74.4 & 127.6 & $\mathrm{~N}$ & 4.1 \\
\hline $2,4,140-142$ & 10.71 & 13.36 & +71.6 & 140.7 & +73.2 & 122.8 & $\mathrm{~N}$ & 9.8 \\
\hline $2,5,20-22$ & 11.01 & 30.81 & +64.1 & 127.0 & +68.4 & 135.4 & $\mathrm{~N}$ & 18.1 \\
\hline $2,5,50-52$ & 11.31 & 24.31 & +83.1 & 121.2 & +80.3 & 128.9 & $\mathrm{~N}$ & 19.2 \\
\hline $2,5,79-81$ & 11.60 & 28.55 & +75.2 & 145.0 & +77.4 & 128.0 & $\mathrm{~N}$ & 20.6 \\
\hline $2,5,110-112$ & 11.91 & 7.68 & +76.3 & 212.8 & +75.8 & 136.0 & $\mathrm{~N}$ & 10.4 \\
\hline $2,6, \quad 4-6$ & 12.35 & 4.35 & -7.1 & 233.1 & -36.0 & 244.7 & & 41.7 \\
\hline $2,6,31-33$ & 12.62 & 6.10 & +65.4 & 72.5 & +69.8 & 109.6 & $\mathrm{~N}$ & 12.8 \\
\hline $2,6,60-62$ & 12.91 & 21.24 & +63.4 & 86.0 & +66.1 & 109.7 & $\mathrm{~N}$ & 18.8 \\
\hline $2,6,92-94$ & 13.23 & 2.41 & +85.3 & 250.2 & +78.7 & 124.1 & $\mathrm{~N}$ & 33.3 \\
\hline $2, C, \quad 8-10$ & 13.59 & 8.57 & -22.5 & 86.5 & -29.3 & 325.5 & & 4.4 \\
\hline $3,1,20-22$ & 14.51 & 44.27 & +58.4 & 105.8 & +73.1 & 179.4 & $\mathrm{~N}$ & 21.4 \\
\hline $3,1,50-52$ & 14.81 & 32.63 & +82.0 & 163.1 & +79.0 & 175.4 & $\mathrm{~N}$ & 21.4 \\
\hline $3,1,80-82$ & 15.11 & 1.47 & +65.3 & 245.2 & +72.0 & 239.8 & $\mathrm{~N}$ & 21.3 \\
\hline $3,1,110-112$ & 15.41 & 21.85 & +81.6 & 275.1 & +79.2 & 261.9 & $\mathrm{~N}$ & 26.2 \\
\hline $3,1,140-142$ & 15.71 & 17.11 & +78.6 & 245.4 & +76.2 & 237.5 & $\mathrm{~N}$ & 19.4 \\
\hline $3,2,20-22$ & 16.01 & 72.82 & +86.1 & 308.9 & +85.1 & 278.2 & $\mathrm{~N}$ & 45.0 \\
\hline
\end{tabular}


Table A1 (continued).

\begin{tabular}{|c|c|c|c|c|c|c|c|c|}
\hline $\begin{array}{l}\text { Core, Section, } \\
\text { Interval }(\mathrm{cm})\end{array}$ & $\begin{array}{l}\text { Depth } \\
\text { (mbsf) }\end{array}$ & $\begin{array}{c}\mathrm{J} \\
\mathrm{NRM} \\
(\mathrm{mA} / \mathrm{m})\end{array}$ & $\begin{array}{l}\text { Incl. } \\
\text { NRM } \\
\text { (deg) }\end{array}$ & $\begin{array}{l}\text { Decl. } \\
\text { NRM } \\
\text { (deg) }\end{array}$ & $\begin{array}{l}\text { Incl } \\
\text { stable } \\
\text { (deg) }\end{array}$ & $\begin{array}{l}\text { Decl. } \\
\text { stable } \\
\text { (deg) }\end{array}$ & Pol. & $\begin{array}{l}\text { MDF } \\
(\mathrm{mT})\end{array}$ \\
\hline $3,2,50-52$ & 16.31 & 8.81 & +80.9 & 158.9 & +70.3 & 252.9 & $\mathrm{~N}$ & 13.6 \\
\hline $3,2,79-81$ & 16.60 & 20.48 & +88.5 & 54.9 & +87.4 & 38.5 & $\mathrm{~N}$ & 22.4 \\
\hline $3,2,110-112$ & 16.91 & 19.82 & +83.7 & 262.0 & +79.9 & 256.4 & $\mathrm{~N}$ & 22.7 \\
\hline $3,2,140-142$ & 17.21 & 30.60 & +77.6 & 213.1 & +75.8 & 231.1 & $\mathrm{~N}$ & 26.2 \\
\hline $3,3,20-22$ & 17.51 & 8.00 & +79.9 & 328.2 & +82.8 & 269.6 & $\mathrm{~N}$ & 23.5 \\
\hline $3,3,50-52$ & 17.81 & 43.36 & +78.0 & 266.9 & +76.5 & 264.2 & $\mathrm{~N}$ & 28.1 \\
\hline $3,3,79-81$ & 18.10 & 24.45 & +85.8 & 349.7 & +82.5 & 251.9 & $\mathrm{~N}$ & 27.1 \\
\hline $3,3,109-111$ & 18.40 & 2.88 & +31.9 & 288.0 & -64.3 & 298.5 & & 42.5 \\
\hline $3,4,19-21$ & 19.00 & 27.40 & +72.5 & 48.5 & +74.4 & 42.0 & $\mathrm{~N}$ & 15.0 \\
\hline $3,4,49-51$ & 19.30 & 68.89 & +67.4 & 66.3 & +69.0 & 62.0 & $\mathrm{~N}$ & 23.4 \\
\hline $3,4,80-82$ & $19.6 \hat{1}$ & 28.31 & +58.2 & 101.4 & +67.5 & 95.5 & $\mathrm{~N}$ & 19.9 \\
\hline $3,4,109-111$ & 19.90 & 50.70 & +67.1 & 83.6 & +67.5 & 84.0 & $\mathrm{~N}$ & 22.6 \\
\hline $4,2,80-82$ & 23.01 & 65.19 & +80.4 & 18.7 & +78.5 & 13.2 & $\mathrm{~N}$ & 31.6 \\
\hline $4,2,110-112$ & 23.31 & 22.82 & +72.7 & 350.9 & +71.3 & 351.5 & $\mathrm{~N}$ & 28.8 \\
\hline $4,2,140-142$ & 23.61 & 65.32 & +84.0 & 62.7 & +86.3 & 59.4 & $\mathrm{~N}$ & 28.0 \\
\hline $4,3,20-22$ & 23.91 & 64.34 & +68.0 & 346.9 & +68.7 & 347.5 & $\mathrm{~N}$ & 30.5 \\
\hline $4,3,49-51$ & 24.20 & 72.92 & +52.3 & .7 & +71.2 & 12.3 & $\mathrm{~N}$ & 26.9 \\
\hline $4,3,79-81$ & 24.50 & 9.52 & +65.9 & 319.2 & +69.6 & 317.9 & $\mathrm{~N}$ & 27.5 \\
\hline $4,3,110-112$ & 24.81 & 18.44 & +70.1 & 350.1 & +68.3 & 348.1 & $\mathrm{~N}$ & 22.3 \\
\hline $4,3,140-142$ & 25.11 & 60.81 & +38.9 & 344.6 & +67.2 & 337.6 & $\mathrm{~N}$ & 29.6 \\
\hline $4,4,19-21$ & 25.40 & 81.11 & +53.1 & 32.3 & +65.5 & 49.4 & $\mathrm{~N}$ & 31.3 \\
\hline $4,4,49-51$ & 25.70 & 92.96 & +48.8 & 3.3 & +64.2 & 32.8 & $\mathrm{~N}$ & 29.2 \\
\hline $4,4,79-81$ & 26.00 & 81.39 & +57.8 & 3.7 & +66.5 & 16.4 & $\mathrm{~N}$ & 31.9 \\
\hline $4,4,110-112$ & 26.31 & 55.39 & +56.0 & 351.4 & +79.7 & 344.7 & $\mathrm{~N}$ & 31.0 \\
\hline $4,5,19-21$ & 26.90 & 5.80 & +72.7 & 38.8 & +72.0 & 40.0 & $\mathrm{~N}$ & 23.7 \\
\hline $4,5,47-49$ & 27.18 & 2.88 & +65.0 & 18.6 & +76.4 & 56.5 & $\mathrm{~N}$ & 11.5 \\
\hline $4,5,79-81$ & 27.50 & 3.13 & +54.8 & 358.7 & +78.6 & 353.2 & $\mathrm{~N}$ & 15.3 \\
\hline $4,5,110-112$ & 27.81 & 21.50 & +75.2 & 19.0 & +75.2 & 9.5 & $\mathrm{~N}$ & 25.6 \\
\hline $4,5,140-142$ & 28.11 & 15.97 & +51.7 & 27.1 & +64.5 & 42.5 & $\mathrm{~N}$ & 26.4 \\
\hline $4,6,19-21$ & 28.40 & 19.98 & +68.9 & 322.3 & +71.4 & 337.9 & $\mathrm{~N}$ & 27.6 \\
\hline $4,6,50-52$ & 28.71 & 29.37 & +77.2 & 325.5 & +67.9 & 328.7 & $\mathrm{~N}$ & 4.4 \\
\hline $4,6,78-80$ & 28.99 & 43.62 & +46.5 & 355.4 & +62.2 & 339.5 & $\mathrm{~N}$ & 32.1 \\
\hline $4,6,110-112$ & 29.31 & 33.73 & +84.4 & 313.8 & +73.2 & 323.5 & $\mathrm{~N}$ & 7.4 \\
\hline $5,1,20-22$ & 29.61 & 62.36 & +74.0 & 145.7 & +74.4 & 148.3 & $\mathrm{~N}$ & 28.9 \\
\hline $5,1,50-52$ & 29.91 & 27.76 & +81.9 & 128.3 & +81.0 & 140.9 & $\mathrm{~N}$ & 27.6 \\
\hline $5,1,80-82$ & 30.21 & 28.65 & +81.0 & 162.4 & +80.5 & 160.2 & $\mathrm{~N}$ & 28.9 \\
\hline $5,1,110-112$ & 30.51 & 32.02 & +67.8 & 124.6 & +68.1 & 132.6 & $\mathrm{~N}$ & 25.5 \\
\hline $5,1,140-142$ & 30.81 & 28.87 & +75.5 & 158.5 & +74.9 & 158.8 & $\mathrm{~N}$ & 29.3 \\
\hline $5,2,20-22$ & 31.11 & 36.56 & +72.3 & 141.0 & +72.4 & 152.0 & $\mathrm{~N}$ & 26.1 \\
\hline $5,2,50-52$ & 31.41 & 37.69 & +78.3 & 113.0 & +81.6 & 136.1 & $\mathrm{~N}$ & 31.1 \\
\hline $5,2,80-82$ & 31.71 & 27.19 & +74.5 & 113.7 & +75.5 & 130.0 & $\mathrm{~N}$ & 29.4 \\
\hline $5,3,19-21$ & 32.60 & 4.53 & +80.7 & 186.5 & +75.5 & 162.9 & $\mathrm{~N}$ & 22.0 \\
\hline $5,3,50-52$ & 32.91 & 4.90 & +76.8 & 129.9 & +79.5 & 152.8 & $\mathrm{~N}$ & 31.0 \\
\hline $5,3,82-84$ & 33.23 & 2.54 & +68.9 & 107.2 & +73.3 & 127.4 & $\mathrm{~N}$ & 22.2 \\
\hline $5,3,110-112$ & 33.51 & 12.43 & +61.5 & 110.1 & +70.1 & 126.0 & $\mathrm{~N}$ & 10.8 \\
\hline $5,3,139-141$ & 33.80 & 23.09 & +73.3 & 117.2 & +73.9 & 132.0 & $\mathrm{~N}$ & 23.8 \\
\hline $5,4,20-22$ & 34.11 & 38.99 & +78.2 & 129.2 & +75.6 & 136.2 & $\mathrm{~N}$ & 21.6 \\
\hline $5,4,50-52$ & 34.41 & 8.61 & +75.3 & 172.7 & +65.8 & 150.2 & $\mathrm{~N}$ & 11.5 \\
\hline
\end{tabular}


Table A1 (continued).

\begin{tabular}{|c|c|c|c|c|c|c|c|c|}
\hline $\begin{array}{l}\text { Core, Section, } \\
\text { Interval }(\mathrm{cm})\end{array}$ & $\begin{array}{l}\text { Depth } \\
\text { (mbsf) }\end{array}$ & $\begin{array}{c}\mathrm{J} \\
\mathrm{NRM} \\
(\mathrm{mA} / \mathrm{m})\end{array}$ & $\begin{array}{l}\text { Incl. } \\
\text { NRM } \\
\text { (deg) }\end{array}$ & $\begin{array}{l}\text { Decl. } \\
\text { NRM } \\
\text { (deg) }\end{array}$ & $\begin{array}{l}\text { Incl } \\
\text { stable } \\
\text { (deg) }\end{array}$ & $\begin{array}{l}\text { Decl. } \\
\text { stable } \\
\text { (deg) }\end{array}$ & Pol. & $\begin{array}{r}\text { MDF } \\
(\mathrm{mT})\end{array}$ \\
\hline $5,4,80-82$ & 34.71 & 10.70 & +67.9 & 144.0 & +73.2 & 143.4 & $\mathrm{~N}$ & 9.0 \\
\hline $5,4,110-112$ & 35.01 & 42.24 & +81.6 & 204.2 & +79.3 & 159.4 & $\mathrm{~N}$ & 24.1 \\
\hline $5,5,20-22$ & 35.61 & 28.85 & +80.4 & 160.1 & +82.0 & 153.2 & $\mathrm{~N}$ & 11.6 \\
\hline $5,5,50-52$ & 35.91 & 66.82 & +81.3 & 132.0 & +76.9 & 141.7 & $\mathrm{~N}$ & 21.2 \\
\hline $5,5,80-82$ & 36.21 & 18.53 & +50.3 & 115.6 & +59.7 & 127.9 & $\mathrm{~N}$ & 15.1 \\
\hline $5,5,110-112$ & 36.51 & 1.82 & +8.3 & 107.3 & +71.3 & 129.4 & $\mathrm{~N}$ & 5.0 \\
\hline $5,5,140-142$ & 36.81 & 11.16 & +74.0 & 296.7 & +80.3 & 165.7 & $\mathrm{~N}$ & 34.8 \\
\hline $5,6,20-22$ & 37.11 & 7.73 & +81.0 & 23.1 & +73.0 & 137.4 & $\mathrm{~N}$ & 25.2 \\
\hline $5,6,49-51$ & 37.40 & 25.83 & -79.2 & 358.0 & -76.3 & 330.8 & $\mathrm{R}$ & 30.8 \\
\hline $5,6,80-82$ & 37.71 & 30.23 & -67.4 & 322.0 & -71.0 & 313.0 & $\mathrm{R}$ & 39.0 \\
\hline $5,6,110-112$ & 38.01 & 33.58 & -57.7 & 289.3 & -60.8 & 290.8 & $\mathrm{R}$ & 32.1 \\
\hline $5,6,140-142$ & 38.31 & 1.16 & +28.5 & 45.6 & -71.9 & 303.4 & $\mathrm{R}$ & 49.9 \\
\hline $5,7,19-21$ & 38.60 & 13.10 & -59.6 & 96.8 & -74.8 & 317.7 & $\mathrm{R}$ & 4.6 \\
\hline $5,7,49-51$ & 38.90 & 6.50 & -16.7 & 284.1 & -59.9 & 308.9 & $\mathrm{R}$ & 19.7 \\
\hline $5,7,68-70$ & 39.09 & 16.24 & -41.5 & 295.0 & -60.4 & 314.5 & $\mathrm{R}$ & 9.9 \\
\hline $6,1,20-22$ & 39.11 & .92 & +26.2 & 243.9 & -69.0 & 219.8 & $\mathrm{R}$ & 46.8 \\
\hline $6,1,50-52$ & 39.41 & 43.60 & -84.3 & 197.8 & -79.4 & 208.0 & $\mathrm{R}$ & 20.4 \\
\hline $6,1,80-82$ & 39.71 & 32.25 & -81.1 & 188.7 & -78.7 & 191.1 & $\mathrm{R}$ & 26.3 \\
\hline $6,1,110-112$ & 40.01 & 15.24 & -75.9 & 256.7 & -79.8 & 225.0 & $\mathrm{R}$ & 16.4 \\
\hline $6,1,140-142$ & 40.31 & 51.62 & -82.1 & 61.4 & -82.1 & 233.6 & $\mathrm{R}$ & 31.5 \\
\hline $6,2,20-22$ & 40.61 & .80 & -73.7 & 168.3 & -74.2 & 210.9 & $R$ & \\
\hline $6,2,50-52$ & 40.91 & 7.07 & -82.7 & 192.1 & -84.6 & 227.2 & $\mathrm{R}$ & 40.6 \\
\hline $5,2,80-82$ & 41.21 & 2.37 & -33.2 & 258.8 & -66.0 & 236.7 & $\mathrm{R}$ & \\
\hline $6,2,110-112$ & 41.51 & 9.63 & +41.2 & 258.6 & -69.9 & 221.6 & $\mathrm{R}$ & 4.8 \\
\hline $6,2,140-142$ & 41.81 & 29.54 & -60.5 & 221.9 & -65.2 & 206.2 & $\mathrm{R}$ & 35.6 \\
\hline $6,3,20-22$ & 42.11 & 22.11 & +66.6 & 97.3 & +67.1 & 95.7 & $\mathrm{~N}$ & 24.3 \\
\hline $6,3,50-52$ & 42.41 & 35.40 & +83.7 & 110.3 & +79.8 & 93.1 & $\mathrm{~N}$ & 20.5 \\
\hline $6,3,80-82$ & 42.71 & 17.26 & -73.8 & 223.0 & +81.7 & 96.4 & $\mathrm{~N}$ & 47.4 \\
\hline $6,3,110-112$ & 43.01 & 10.10 & -74.2 & 105.0 & +65.3 & 107.8 & $\mathrm{~N}$ & 3.7 \\
\hline $6,3,140-142$ & 43.31 & .83 & +20.7 & 109.4 & +70.3 & 113.4 & $\mathrm{~N}$ & \\
\hline $6,4,20-22$ & 43.61 & 15.33 & +73.0 & 81.0 & +61.8 & 94.6 & $\mathrm{~N}$ & 9.7 \\
\hline $6,4,50-52$ & 43.91 & 8.92 & +78.0 & 70.5 & +66.8 & 76.8 & $\mathrm{~N}$ & 8.1 \\
\hline $6,4,80-82$ & 44.21 & 13.78 & -1.7 & 86.3 & +61.2 & 72.9 & $\mathrm{~N}$ & 24.7 \\
\hline $6,4,110-112$ & 44.51 & 22.21 & +81.8 & 58.8 & +85.1 & 66.5 & $\mathrm{~N}$ & 39.1 \\
\hline $6,5,20-22$ & 45.11 & 12.10 & +69.0 & 48.6 & +65.3 & 50.6 & $\mathrm{~N}$ & 26.5 \\
\hline $6,5,50-52$ & 45.41 & 3.14 & +57.0 & 96.2 & +17.2 & 109.0 & & 4.4 \\
\hline $6,5,80-82$ & 45.71 & 9.44 & -25.8 & 251.2 & -61.1 & 249.6 & $\mathrm{R}$ & \\
\hline $6,5,110-112$ & 46.01 & 6.49 & +31.5 & 257.7 & -71.5 & 257.6 & $\mathrm{R}$ & 24.2 \\
\hline $6,5,140-142$ & 46.31 & 16.00 & -51.8 & 297.1 & -63.5 & 264.7 & $\mathrm{R}$ & 35.4 \\
\hline $6,6,20-22$ & 46.61 & .82 & -4.5 & 76.6 & -69.5 & 258.7 & $\mathrm{R}$ & 36.3 \\
\hline $6,6,50-52$ & 46.91 & 10.95 & +57.6 & 70.4 & -69.6 & 230.7 & $\mathrm{R}$ & 3.5 \\
\hline $7,1,109-111$ & 48.50 & 6.34 & +27.0 & 303.9 & -15 & 27 & $\mathrm{R}$ & 29.8 \\
\hline $7,1,139-141$ & 48.80 & 1.84 & -38.7 & 250.7 & -71.9 & 254.9 & $\mathrm{R}$ & 15.7 \\
\hline $7,2,8-10$ & 48.99 & 32.13 & -63.3 & 254.8 & -67.3 & 254.7 & $\mathrm{R}$ & 32.0 \\
\hline $7,2,38-40$ & 49.29 & 22.27 & -70.1 & 280.3 & -74.6 & 276.5 & $\mathrm{R}$ & 29.4 \\
\hline $7,2,68-70$ & 49.59 & 3.72 & +9.2 & 158.5 & -66.6 & 223.6 & $\mathrm{R}$ & 53.7 \\
\hline $7,2,99-101$ & 49.90 & 34.55 & -74.9 & 230.8 & -73.0 & 237.7 & $\mathrm{R}$ & 32.0 \\
\hline $7,2,139-141$ & 50.30 & 27.03 & -78.8 & 298.6 & -75.6 & 273.9 & $\mathrm{R}$ & 33.5 \\
\hline
\end{tabular}


Table A1 (continued).

\begin{tabular}{|c|c|c|c|c|c|c|c|c|}
\hline $\begin{array}{l}\text { Core, Section, } \\
\text { Interval }(\mathrm{cm})\end{array}$ & $\begin{array}{l}\text { Depth } \\
\text { (mbsf) }\end{array}$ & $\underset{(\mathrm{mA} / \mathrm{m})}{\mathrm{J}}$ & $\begin{array}{l}\text { Incl. } \\
\text { NRM } \\
\text { (deg) }\end{array}$ & $\begin{array}{l}\text { Decl. } \\
\text { NRM } \\
\text { (deg) }\end{array}$ & $\begin{array}{c}\text { Incl } \\
\text { stable } \\
\text { (deg) }\end{array}$ & $\begin{array}{l}\text { Decl. } \\
\text { stable } \\
\text { (deg) }\end{array}$ & Pol. & $\begin{array}{l}\mathrm{MDF} \\
(\mathrm{mT})\end{array}$ \\
\hline $7,3,8-10$ & 50.49 & 16.71 & -71.0 & 273.7 & -75.8 & 269.9 & $\mathrm{R}$ & 33.4 \\
\hline $7,3,38-40$ & 50.79 & 33.83 & -68.9 & 269.9 & -76.3 & 264.5 & $\mathrm{R}$ & 32.6 \\
\hline $7,3,68-70$ & 51.09 & 27.19 & -63.3 & 268.0 & -68.8 & 249.7 & $\mathrm{R}$ & 9.4 \\
\hline $7,3,99-101$ & 51.40 & 13.79 & -60.5 & 206.1 & -74.3 & 221.7 & $\mathrm{R}$ & 38.3 \\
\hline $7,3,139-141$ & 51.80 & 4.39 & -10.8 & 266.2 & -70.5 & 246.7 & $\mathrm{R}$ & \\
\hline $7,4, \quad 8-10$ & 51.99 & 10.55 & -52.3 & 278.1 & -63.3 & 255.9 & $\mathrm{R}$ & 47.1 \\
\hline $7,4,38-40$ & 52.29 & .64 & +45.7 & 200.3 & -62.7 & 230.0 & $\mathrm{R}$ & 38.3 \\
\hline $7,4,68-70$ & 52.59 & 36.02 & -76.7 & 223.4 & -77.5 & 236.4 & $\mathrm{R}$ & 32.2 \\
\hline $7,4,99-101$ & 52.90 & 2.81 & -30.6 & 128.0 & +58.1 & 52.0 & $\mathrm{~N}$ & 19.8 \\
\hline $7,5,8-10$ & 53.49 & .68 & +39.6 & 109.6 & +66.0 & 91.2 & $\mathrm{~N}$ & 4.9 \\
\hline $7,5,39-41$ & 53.80 & 24.38 & +82.1 & 91.2 & +80.6 & 92.6 & $\mathrm{~N}$ & 24.2 \\
\hline $7,5,68-70$ & 54.09 & 38.45 & +82.3 & 44.5 & +83.0 & 85.0 & $\mathrm{~N}$ & 30.9 \\
\hline $7,5,99-101$ & 54.40 & 35.63 & +73.6 & 55.6 & +73.1 & 60.9 & $\mathrm{~N}$ & 30.2 \\
\hline $7,5,139-141$ & 54.80 & 10.56 & +83.0 & 73.3 & +83.1 & 70.8 & $\mathrm{~N}$ & 14.9 \\
\hline $7,6, \quad 8-10$ & 54.99 & 46.73 & +73.0 & 43.5 & +72.2 & 30.9 & N & 21.5 \\
\hline $7,6,39-41$ & 55.30 & 7.16 & -8.2 & 252.9 & -52.8 & 217.6 & $\mathrm{R}$ & 30.5 \\
\hline $7,6,68-70$ & 55.59 & 10.62 & -45.0 & 263.9 & -63.5 & 257.1 & $\mathrm{R}$ & 34.1 \\
\hline $7,6,99-101$ & 55.90 & 1.01 & -5.8 & 233.2 & -63.0 & 228.9 & $\mathrm{R}$ & \\
\hline $7,6,139-141$ & 56.30 & 2.87 & -29.8 & 296.6 & -65.6 & 254.2 & $\mathrm{R}$ & \\
\hline $7,7, \quad 8-10$ & 56.49 & 9.20 & -70.3 & 190.6 & -70.6 & 204.0 & $\mathrm{R}$ & 47.2 \\
\hline $7,7,39-41$ & 56.80 & 24.60 & +31.9 & 100.9 & +38.2 & 99.7 & & 18.2 \\
\hline $7,7,68-70$ & 57.09 & .77 & -32.8 & 65.0 & -76.3 & 316.9 & $\mathrm{R}$ & 30.2 \\
\hline $8,1,39-41$ & 57.30 & 18.75 & +69.5 & 50.1 & +67.4 & & $\mathrm{~N}$ & 17.0 \\
\hline $8,1,69-71$ & 57.60 & 1.08 & +74.7 & 119.0 & +72.8 & 309.6 & $\mathrm{~N}$ & 3.9 \\
\hline $8,1,99-101$ & 57.90 & 5.46 & +4.7 & 219.9 & -58.2 & 215.1 & $\mathrm{R}$ & \\
\hline $8,1,129-131$ & 58.20 & .75 & -59.0 & 351.4 & -71.6 & 298.2 & $\mathrm{R}$ & \\
\hline $8,2,9-11$ & 58.50 & 6.72 & -36.4 & 282.1 & -60.1 & 278.6 & $\mathrm{R}$ & 48.6 \\
\hline $8,2,39-41$ & 58.80 & 12.65 & -40.1 & 291.4 & -66.4 & 293.6 & $\mathrm{R}$ & 49.3 \\
\hline $8,2,69-71$ & 59.10 & 22.80 & -48.8 & 282.3 & -69.1 & 286.6 & $\mathrm{R}$ & \\
\hline $8,2,99-101$ & 59.40 & 8.49 & -37.2 & 283.0 & -63.7 & 280.8 & $\mathrm{R}$ & 48.6 \\
\hline $8,2,129-131$ & 59.70 & 20.83 & -74.0 & 290.4 & -73.7 & 291.9 & $\mathrm{R}$ & 38.1 \\
\hline $8,3,9-11$ & 60.00 & 2.50 & -76.0 & 344.4 & -64.5 & 297.4 & $\mathrm{R}$ & 4.9 \\
\hline $8,3,43-45$ & 60.34 & 15.62 & -79.1 & 313.9 & -79.4 & 307.0 & $\mathrm{R}$ & 39.5 \\
\hline $8,3,73-75$ & 60.64 & 17.04 & -62.2 & 271.6 & -71.5 & 275.1 & $\mathrm{R}$ & 35.9 \\
\hline $8,3,99-101$ & 60.90 & 1.57 & -32.7 & 274.6 & -65.5 & 272.9 & $\mathrm{R}$ & \\
\hline $8,3,129-131$ & 61.20 & 9.24 & -54.3 & 287.0 & -61.5 & 294.9 & $\mathrm{R}$ & 38.4 \\
\hline $8,4,9-11$ & 61.50 & 7.44 & +34.6 & 284.7 & -73.1 & 307.5 & $\mathrm{R}$ & 47.2 \\
\hline $8,4,39-41$ & 61.80 & 4.15 & -42.2 & 342.5 & -68.2 & 303.3 & $\mathrm{R}$ & \\
\hline $8,4,69-71$ & 62.10 & 13.09 & -25.0 & 106.5 & -71.5 & 308.6 & $\mathrm{R}$ & 8.6 \\
\hline $8,4,99-101$ & 62.40 & 10.10 & +67.5 & 249.6 & -73.4 & 305.8 & $\mathrm{R}$ & 3.5 \\
\hline $8,4,129-131$ & 62.70 & 8.64 & -78.5 & 281.7 & -69.3 & 285.7 & $\mathrm{R}$ & 33.3 \\
\hline $8,5,9-11$ & 63.00 & 8.41 & -55.9 & .0 & -67.9 & 309.9 & $\mathrm{R}$ & 39.3 \\
\hline $8,5,39-41$ & 63.30 & 27.07 & -85.0 & 25.7 & -81.3 & 301.9 & $\mathrm{R}$ & 29.0 \\
\hline $8,5,69-71$ & 63.60 & 14.33 & -67.2 & 73.6 & -74.2 & 282.1 & $\mathrm{R}$ & 32.6 \\
\hline $8,5,99-101$ & 63.90 & 4.27 & -37.2 & 24.3 & -65.7 & 312.9 & $\mathrm{R}$ & 48.1 \\
\hline $8,5,131-133$ & 64.22 & 2.81 & +69.5 & 271.8 & -47.4 & 289.5 & $\mathrm{R}$ & 7.7 \\
\hline $8,6,9-11$ & 64.50 & 1.44 & -77.3 & 324.5 & -77.5 & 318.7 & $\mathrm{R}$ & 70.9 \\
\hline $8.6,39-41$ & 64.80 & 5.75 & +66.1 & 126.4 & +72.0 & 140.2 & $\mathrm{~N}$ & 6.7 \\
\hline $8,6,69-71$ & 65.10 & 18.05 & +61.5 & 126.5 & +71.1 & 100.1 & $\mathrm{~N}$ & 13.5 \\
\hline
\end{tabular}


Table A1 (continued).

\begin{tabular}{|c|c|c|c|c|c|c|c|c|}
\hline $\begin{array}{l}\text { Core, Section, } \\
\text { Interval }(\mathrm{cm})\end{array}$ & $\begin{array}{l}\text { Depth } \\
\text { (mbsf) }\end{array}$ & $\begin{array}{c}\mathrm{J} \\
\mathrm{NRM} \\
(\mathrm{mA} / \mathrm{m})\end{array}$ & $\begin{array}{l}\text { Incl. } \\
\text { NRM } \\
\text { (deg) }\end{array}$ & $\begin{array}{l}\text { Decl. } \\
\text { NRM } \\
\text { (deg) }\end{array}$ & $\begin{array}{c}\text { Incl } \\
\text { stable } \\
\text { (deg) }\end{array}$ & $\begin{array}{l}\text { Decl. } \\
\text { stable } \\
\text { (deg) }\end{array}$ & Pol. & $\begin{array}{l}\text { MDF } \\
(\mathrm{mT})\end{array}$ \\
\hline $8,6,99-101$ & 65.40 & 7.41 & +74.8 & 110.3 & +71.5 & 112.2 & $\mathrm{~N}$ & 14.5 \\
\hline $8,6,129-131$ & 65.70 & 4.23 & +73.4 & 96.3 & +78.8 & 99.4 & $\mathrm{~N}$ & 12.9 \\
\hline $8,7,9-11$ & 66.00 & 46.62 & +35.3 & 171.1 & +73.2 & 115.9 & $\mathrm{~N}$ & 7.5 \\
\hline $8,7,39-41$ & 66.30 & 18.02 & +73.5 & 89.9 & +69.2 & 87.8 & $\mathrm{~N}$ & 14.2 \\
\hline $9,1,10-12$ & 66.51 & 4.76 & +75.9 & 104.5 & +69.7 & 38.7 & $\mathrm{~N}$ & 4.7 \\
\hline $9,1,40-42$ & 66.81 & 31.44 & +82.7 & 43.3 & +83.0 & 61.2 & $\mathrm{~N}$ & 27.5 \\
\hline $9,1,70-72$ & 67.11 & 4.76 & -76.6 & 145.6 & -74.7 & 202.3 & $\mathrm{R}$ & \\
\hline $9,1,100-102$ & 67.41 & 10.75 & -65.8 & 202.3 & -74.7 & 203.6 & $\mathrm{R}$ & \\
\hline $9,1,130-132$ & 67.71 & 9.07 & -30.7 & 236.5 & -73.6 & 196.9 & $\mathrm{R}$ & 43.0 \\
\hline $9,2,10-12$ & 68.01 & 10.23 & -72.4 & 135.0 & -65.4 & 191.1 & $\mathrm{R}$ & 45.7 \\
\hline $9,2,40-42$ & 68.31 & 4.34 & +76.5 & 101.5 & +80.4 & 96.6 & $\mathrm{~N}$ & \\
\hline $9,2,70-72$ & 68.61 & 14.97 & +72.8 & 14.0 & +67.8 & 15.0 & $\mathrm{~N}$ & 25.4 \\
\hline $9,2,100-102$ & 68.91 & 8.18 & +67.7 & 56.5 & +65.3 & 34.9 & $\mathrm{~N}$ & 13.3 \\
\hline $9,2,130-132$ & 69.21 & 12.26 & +72.8 & 30.8 & +67.4 & 37.1 & $\mathrm{~N}$ & 17.2 \\
\hline $9,3,10-12$ & 69.51 & 8.14 & +73.2 & 99.8 & +78.4 & 65.4 & $\mathrm{~N}$ & 27.2 \\
\hline $9,3,46-48$ & 69.87 & 9.27 & +67.9 & 50.8 & +66.0 & 47.0 & $\mathrm{~N}$ & 7.4 \\
\hline $9,3,70-72$ & 70.11 & 2.18 & -3.6 & 260.2 & -79.9 & 264.1 & $\mathrm{R}$ & \\
\hline $9,3,100-102$ & 70.41 & 11.38 & -59.8 & 160.3 & -81.5 & 194.4 & $\mathrm{R}$ & \\
\hline $9,4,11-13$ & 71.02 & 10.45 & -58.3 & 145.4 & -72.3 & 177.4 & $\mathrm{R}$ & \\
\hline $9,4,40-42$ & 71.31 & 10.56 & -54.9 & 162.3 & -71.3 & 184.2 & $\mathrm{R}$ & \\
\hline $9,4,70-72$ & 71.61 & 13.97 & .74 .8 & 123.8 & -76.9 & 182.4 & $\mathrm{R}$ & 44.7 \\
\hline $9,4,102-104$ & 71.93 & 15.41 & -65.3 & 202.0 & -73.4 & 199.6 & $\mathrm{R}$ & 48.3 \\
\hline $9,4,131-133$ & 72.22 & 15.37 & -55.1 & 161.1 & -69.6 & 173.1 & $\mathrm{R}$ & 48.4 \\
\hline $9,5,11-13$ & 72.52 & 13.01 & -47.9 & 175.9 & -67.4 & 187.0 & $\mathrm{R}$ & 48.3 \\
\hline $9,5,41-43$ & 72.82 & 16.76 & -61.0 & 177.0 & -71.1 & 183.5 & $\mathrm{R}$ & 46.2 \\
\hline $9,5,71-73$ & 73.12 & 17.65 & -56.8 & 160.6 & -69.0 & 167.3 & $\mathrm{R}$ & 42.2 \\
\hline $9,5,100-102$ & 73.41 & 16.35 & -62.2 & 154.1 & -69.1 & 175.7 & $\mathrm{R}$ & 44.0 \\
\hline $9,5,131-133$ & 73. & 35.52 & -68.1 & 176.5 & -72.3 & 177.5 & $\mathrm{R}$ & 31.4 \\
\hline $9,6,10-12$ & 74. & 26.65 & -69.2 & 194.8 & -73.6 & 191.9 & $\mathrm{R}$ & 34.4 \\
\hline $9,6,39-41$ & 74.30 & 24.62 & -72.3 & 205.3 & -76.0 & 199.8 & $\mathrm{R}$ & 40.5 \\
\hline $9,6,70-72$ & 74.61 & 20.44 & -65.9 & 172.8 & -71.6 & 187.0 & $\mathrm{R}$ & 39.6 \\
\hline $9,6,99-101$ & 74. & 12. & -57.1 & 160.3 & -71.2 & 164.4 & $\mathrm{R}$ & 43.8 \\
\hline $9,6,130-132$ & 75. & 23. & -77.1 & 141.0 & -76.8 & 161.0 & $\mathrm{R}$ & 38.4 \\
\hline $9,7,9-11$ & 75.50 & 18 & -79.5 & 141.2 & -78.6 & 165.1 & $\mathrm{R}$ & 42.8 \\
\hline $9,7,31-33$ & 75. & 17. & -62.2 & 206.4 & -68.6 & 197.4 & $\mathrm{R}$ & 40.9 \\
\hline $9,7,55-57$ & 75.96 & 25.23 & -68.3 & 179.7 & -71.4 & 187.1 & $\mathrm{R}$ & 37.8 \\
\hline $10,1, \quad 8-10$ & 7 & 7 & & 74 & & & $\mathrm{~N}$ & 25.1 \\
\hline $10,1,69-71$ & 76.60 & 10.38 & +74.3 & 72.5 & +74.7 & 90.0 & $\mathrm{~N}$ & 10.5 \\
\hline $10,1,100-102$ & 76.91 & 2.72 & +61.7 & 6.6 & -67.9 & 268.3 & $\mathrm{R}$ & 6.1 \\
\hline $10,1,129-131$ & 77.20 & 4.86 & -74.7 & 243.3 & -75.4 & 261.9 & $\mathrm{R}$ & 35.5 \\
\hline $10,2,10-12$ & 77.51 & .60 & +64.8 & 130.9 & +79.9 & 102.6 & $\mathrm{~N}$ & 4.1 \\
\hline $10,2,40-42$ & 77.81 & 2.99 & -79.7 & 216.9 & -78.3 & 244.6 & $\mathrm{R}$ & \\
\hline $10,2,68-70$ & 78.09 & 4.71 & -76.0 & 55.9 & -83.5 & 205.2 & $\mathrm{R}$ & \\
\hline $10,2,100-102$ & 78.41 & 7.68 & -77.6 & 212.3 & -78.1 & 204.9 & $\mathrm{R}$ & 55.1 \\
\hline $10,2,130-132$ & 78.71 & 8.95 & -78.8 & 203.2 & -80.0 & 232.6 & $\mathbf{R}$ & 53.4 \\
\hline $10,3,10-12$ & 79.01 & .13 & +52.9 & 124.2 & -68.2 & 210.0 & $\mathrm{R}$ & 3.8 \\
\hline $10,3,40-42$ & 79.3 & .07 & +61.0 & 214.7 & -60.9 & 222.5 & $\mathbf{R}$ & 4.7 \\
\hline $10,3,70-72$ & 79.61 & .13 & +72.2 & 19.5 & -52.4 & 165.3 & $\mathrm{R}$ & 3.1 \\
\hline
\end{tabular}


Table A1 (continued).

\begin{tabular}{|c|c|c|c|c|c|c|c|c|}
\hline $\begin{array}{l}\text { Core, Section, } \\
\text { Interval }(\mathrm{cm})\end{array}$ & $\begin{array}{l}\text { Depth } \\
\text { (mbsf) }\end{array}$ & $\begin{array}{c}\mathrm{J} \\
\mathrm{NRM} \\
(\mathrm{mA} / \mathrm{m})\end{array}$ & $\begin{array}{l}\text { Incl. } \\
\text { NRM } \\
\text { (deg) }\end{array}$ & $\begin{array}{l}\text { Decl. } \\
\text { NRM } \\
\text { (deg) }\end{array}$ & $\begin{array}{c}\text { Incl } \\
\text { stable } \\
\text { (deg) }\end{array}$ & $\begin{array}{l}\text { Decl. } \\
\text { stable } \\
\text { (deg) }\end{array}$ & Pol. & $\begin{array}{l}\mathrm{MDF} \\
(\mathrm{mT})\end{array}$ \\
\hline $10,3,99-101$ & 79.90 & .26 & +64.4 & 100.9 & +65.2 & 90.4 & N & 7.3 \\
\hline $10,3,130-132$ & 80.21 & .33 & +70.7 & 71.4 & +64.9 & 69.8 & $\mathrm{~N}$ & 9.0 \\
\hline $10,4,10-12$ & 80.51 & 1.30 & +20.4 & 293.6 & +73.8 & 36.8 & $\mathrm{~N}$ & 3.0 \\
\hline $10,4,40-42$ & 80.81 & 1.19 & +36.6 & 272.1 & +73.0 & 33.7 & N & 3.4 \\
\hline $10,4,70-72$ & 81.11 & .50 & +78.8 & 56.9 & +76.2 & 23.6 & $\mathrm{~N}$ & 4.6 \\
\hline $10,4,99-101$ & 81.40 & .78 & +76.6 & 57.3 & +71.4 & 55.9 & $\mathrm{~N}$ & 8.3 \\
\hline $10,4,125-127$ & 81.66 & .78 & +72.2 & 2.5 & +74.6 & 83.1 & $\mathrm{~N}$ & 3.7 \\
\hline $10,5,10-12$ & 82.01 & .32 & +77.2 & 135.0 & -66.9 & 34.8 & $\mathrm{R}$ & 3.0 \\
\hline $10,5,40-42$ & 82.31 & .82 & -54.8 & 169.3 & -75.2 & 205.1 & $\mathrm{R}$ & 62.4 \\
\hline $10,5,69-71$ & 82.60 & 1.42 & -66.8 & 266.0 & -79.1 & 223.0 & $R$ & 17.3 \\
\hline $10,5,98-100$ & 82.89 & .25 & +40.1 & 209.7 & -83.7 & 225.3 & $\mathrm{R}$ & 3.1 \\
\hline $10,5,129-131$ & 83.20 & .23 & +16.7 & 318.3 & -55.4 & 241.4 & $\mathrm{R}$ & 17.6 \\
\hline $10,6,9-11$ & 83.50 & .47 & +27.9 & 56.5 & +66.4 & 79.8 & $\mathrm{~N}$ & 15.2 \\
\hline $10,6,40-42$ & 83.81 & .68 & +76.7 & 355.5 & +71.4 & 21.3 & $\mathrm{~N}$ & 7.4 \\
\hline $10,6,70-72$ & 84.11 & .75 & +78.1 & 79.4 & +74.3 & 57.7 & $\mathrm{~N}$ & 4.6 \\
\hline $10,6,99-101$ & 84.40 & & & & -71.6 & 227.7 & $\mathrm{R}$ & 99.9 \\
\hline $10,6,129-131$ & 84.70 & .09 & +49.7 & 134.8 & -66.7 & 225.2 & $\mathrm{R}$ & 48.7 \\
\hline $10,7,10-12$ & 85.01 & .14 & +72.4 & 18.3 & -80.7 & 254.5 & $\mathrm{R}$ & 6.3 \\
\hline $10,7,40-42$ & 85.31 & .32 & +49.4 & 300.2 & -69.9 & 209.7 & $\mathrm{R}$ & 3.3 \\
\hline $11,1,10-12$ & 85.51 & .16 & -7.8 & 42.7 & -71.5 & 130.8 & $\mathrm{R}$ & 7.9 \\
\hline $11,1,40-42$ & 85.81 & .04 & -38.6 & 165.8 & -71.7 & 161.3 & $\mathrm{R}$ & \\
\hline $11,1,74-76$ & 86.15 & .18 & +57.8 & 190.2 & -78.9 & 277.6 & $\mathrm{R}$ & \\
\hline $11,1,100-102$ & 86.41 & .24 & +39.3 & 192.4 & -80.7 & 159.9 & $\mathrm{R}$ & \\
\hline $11,1,130-132$ & 86.71 & 4.06 & -70.4 & 227.3 & -66.8 & 169.9 & $\mathrm{R}$ & 49.2 \\
\hline $11,2,10-12$ & 87.01 & 5.18 & -55.5 & 134.6 & -63.4 & 148.0 & $\mathrm{R}$ & \\
\hline $11,2,40-42$ & 87.31 & .56 & -64.9 & 116.2 & -67.0 & 157.6 & $\mathrm{R}$ & \\
\hline $11,2,70-72$ & 87.61 & .11 & +29.3 & 176.4 & -65.1 & 143.5 & $\mathrm{R}$ & \\
\hline $11,2,100-102$ & 87.91 & .18 & -8.9 & 150.5 & -66.7 & 159.6 & $\mathrm{R}$ & \\
\hline $11,2,130-132$ & 88.21 & .10 & -66.2 & 6.2 & -75.0 & 142.8 & $\mathrm{R}$ & \\
\hline $11,3,10-12$ & 88.51 & .47 & +59.9 & 111.1 & -65.2 & 148.0 & $\mathrm{R}$ & \\
\hline $11,3,40-42$ & 88.81 & .21 & +64.8 & 80.2 & -62.3 & 157.4 & $\mathrm{R}$ & \\
\hline $11,3,70-72$ & 89.11 & .22 & -71.1 & 77.0 & -72.8 & 158.3 & $\mathrm{R}$ & \\
\hline $11,3,100-102$ & 89.41 & .40 & -57.0 & 76.2 & -71.5 & 158.4 & R & \\
\hline $11,3,130-132$ & 89.71 & .48 & +4.0 & 80.4 & -69.4 & 257.0 & $\mathrm{R}$ & 19.0 \\
\hline $11,4,10-12$ & 90.01 & 1.04 & -33.0 & 117.2 & -70.1 & 173.5 & $\mathrm{R}$ & 43.1 \\
\hline $11,4,40-42$ & 90.31 & 2.62 & -58.8 & 90.8 & -77.1 & 149.4 & $\mathrm{R}$ & \\
\hline $11,4,70-72$ & 90.61 & .23 & -32.0 & 140.9 & -73.9 & 160.8 & $\mathrm{R}$ & \\
\hline $11,4,100-102$ & 90.91 & .10 & -63.8 & 172.2 & -72.6 & 141.8 & $\mathrm{R}$ & \\
\hline $11,4,130-132$ & 91.21 & .13 & -14.3 & 169.0 & -80.3 & 132.7 & $\mathrm{R}$ & \\
\hline $11,5,10-12$ & 91.51 & .84 & -3.8 & 30.9 & -67.7 & 123.9 & $\mathrm{R}$ & 11.2 \\
\hline $11,5,40-42$ & 91.81 & 1.76 & +45.7 & 112.8 & -67.4 & 131.0 & $\mathrm{R}$ & 3.1 \\
\hline $11,5,70-72$ & 92.11 & 10.09 & -73.6 & 158.6 & -74.8 & 165.8 & $\mathrm{R}$ & 42.0 \\
\hline $11,5,100-102$ & 92.41 & 5.82 & +16.3 & 67.2 & -69.3 & 123.1 & $R$ & 3.6 \\
\hline $11,5,130-132$ & 92.71 & .38 & +.9 & 329.1 & -76.4 & 126.8 & $\mathrm{R}$ & 4.7 \\
\hline $11,6,10-12$ & 93.01 & .59 & -35.1 & 287.3 & -67.7 & 133.6 & $\mathrm{R}$ & 30.9 \\
\hline $11,6,40-42$ & 93.31 & .64 & -60.2 & 231.8 & -66.5 & 121.9 & $\mathrm{R}$ & 10.4 \\
\hline $11,6,70-72$ & 93.61 & .44 & -28.0 & 100.6 & -80.6 & 134.6 & $\mathrm{R}$ & \\
\hline $11,6,100-102$ & 93.91 & 10.10 & -18.3 & 117.6 & -66.2 & 154.4 & $\mathrm{R}$ & 6.2 \\
\hline $11,6,130-132$ & 94.21 & 2.74 & +2.1 & 90.7 & -71.5 & 118.3 & $R$ & 5.6 \\
\hline
\end{tabular}


Table A1 (continued).

\begin{tabular}{|c|c|c|c|c|c|c|c|c|}
\hline $\begin{array}{l}\text { Core, Section, } \\
\text { Interval }(\mathrm{cm})\end{array}$ & $\begin{array}{l}\text { Depth } \\
\text { (mbsf) }\end{array}$ & $\begin{array}{c}\mathrm{J} \\
\mathrm{NRM} \\
(\mathrm{mA} / \mathrm{m})\end{array}$ & $\begin{array}{l}\text { Incl. } \\
\text { NRM } \\
\text { (deg) }\end{array}$ & $\begin{array}{l}\text { Decl. } \\
\text { NRM } \\
\text { (deg) }\end{array}$ & $\begin{array}{c}\text { Incl } \\
\text { stable } \\
\text { (deg) }\end{array}$ & $\begin{array}{l}\text { Decl. } \\
\text { stable } \\
\text { (deg) }\end{array}$ & Pol. & $\begin{array}{l}\mathrm{MDF} \\
(\mathrm{mT})\end{array}$ \\
\hline $11,7,10-12$ & 94.51 & 2.05 & +38.6 & 51.9 & -62.7 & 117.4 & $\mathrm{R}$ & 4.2 \\
\hline $11,7,40-42$ & 94.81 & 2.14 & +79.2 & 343.5 & -71.4 & 142.1 & $\mathrm{R}$ & 2.7 \\
\hline $11,7,63-65$ & 95.04 & .20 & -80.3 & 88.8 & -86.4 & 125.3 & $\mathrm{R}$ & \\
\hline $12,1,25-27$ & 95.16 & .57 & +25.0 & 257.8 & +66.2 & 214.1 & $\mathrm{~N}$ & 3.1 \\
\hline $12,1,48-50$ & 95.39 & .59 & +36.6 & 240.7 & +64.4 & 217.3 & $\mathrm{~N}$ & 2.9 \\
\hline $12,1,70-72$ & 95.61 & .26 & +69.2 & 37.8 & +41.7 & 115.0 & $\mathrm{~N}$ & 4.5 \\
\hline $12,1,100-102$ & 95.91 & .17 & +14.6 & 53.2 & -55.4 & 34.7 & $\mathrm{R}$ & \\
\hline $12,2,25-27$ & 96.66 & .81 & -20.7 & 88.2 & -71.9 & 79.0 & $\mathrm{R}$ & \\
\hline $12,2,56-58$ & 96.97 & .59 & +52.9 & 91.5 & -63.2 & 85.9 & $\mathrm{R}$ & 4.4 \\
\hline $12,2,70-72$ & 97.11 & .36 & +59.5 & 20.8 & -70.1 & 65.0 & $\mathrm{R}$ & 5.0 \\
\hline $12,2,100-102$ & 97.41 & .57 & +78.5 & 83.9 & +77.3 & 140.8 & $\mathrm{~N}$ & 4.6 \\
\hline $12,2,130-132$ & 97.71 & .94 & +80.4 & 228.0 & +84.4 & 178.4 & $\mathrm{~N}$ & 9.9 \\
\hline $12,3,10-12$ & 98.01 & .44 & +78.5 & 142.2 & +73.4 & 174.8 & $\mathrm{~N}$ & 4.8 \\
\hline $12,3,36-38$ & 98.27 & .44 & +79.0 & 159.3 & +85.6 & 181.2 & $\mathrm{~N}$ & 7.1 \\
\hline $12,3,70-72$ & 98.61 & .34 & +82.1 & 179.6 & +72.4 & 190.2 & $\mathrm{~N}$ & 3.7 \\
\hline $12,3,100-102$ & 98.91 & 1.35 & +82.1 & 93.8 & +85.7 & 200.6 & $\mathrm{~N}$ & 4.3 \\
\hline $12,3,130-132$ & 99.21 & .87 & +79.8 & 128.5 & +75.2 & 183.7 & $\mathrm{~N}$ & 7.7 \\
\hline $12,4,10-12$ & 99.51 & 1.48 & +78.6 & 107.6 & +72.4 & 181.0 & $\mathrm{~N}$ & 24.2 \\
\hline $12,4,43-45$ & 99.84 & .87 & +79.0 & 141.7 & +75.6 & 169.5 & $\mathrm{~N}$ & 24.0 \\
\hline $12,4,72-74$ & 100.13 & .29 & +69.2 & 267.9 & +77.2 & 207.3 & $\mathrm{~N}$ & 4.1 \\
\hline $12,4,102-104$ & 100.43 & .31 & +83.4 & 132.9 & +78.7 & 236.0 & $\mathrm{~N}$ & 4.0 \\
\hline $12,5,10-12$ & 101.01 & 1.03 & +82.3 & 334.6 & +85.6 & 191.6 & $\mathrm{~N}$ & 25.8 \\
\hline $12,5,43-45$ & 101.34 & .51 & +86.9 & 129.3 & +83.8 & 216.4 & $\mathrm{~N}$ & 17.7 \\
\hline $12,5,72-74$ & 101.63 & .38 & +79.6 & 58.4 & +70.7 & 184.1 & $\mathrm{~N}$ & 16.0 \\
\hline $12,5,102-104$ & 101.93 & .38 & +86.9 & 41.3 & +80.9 & 227.9 & $\mathrm{~N}$ & 5.0 \\
\hline $12,5,134-136$ & 102.25 & .81 & +84.6 & 136.3 & +78.4 & 196.8 & $\mathrm{~N}$ & 6.3 \\
\hline $12,6,10-12$ & 102.51 & 1.00 & +82.0 & 161.0 & +76.8 & 176.7 & $\mathrm{~N}$ & 8.6 \\
\hline $12,6,43-45$ & 102.84 & 1.15 & +86.4 & 196.6 & +82.4 & 202.5 & $\mathrm{~N}$ & 15.8 \\
\hline $12,6,72-74$ & 103.13 & 1.07 & +86.4 & 229.2 & +77.4 & 210.9 & $\mathrm{~N}$ & 15.2 \\
\hline $12,6,102-104$ & 103.43 & .63 & +85.4 & 344.4 & +74.8 & 194.1 & $\mathrm{~N}$ & 16.4 \\
\hline $12,6,134-136$ & 103.75 & .40 & +61.1 & 57.1 & +72.4 & 225.7 & $\mathrm{~N}$ & 6.2 \\
\hline $12,7,10-12$ & 104.01 & .47 & +72.1 & 124.2 & +66.5 & 199.7 & $\mathrm{~N}$ & 4.5 \\
\hline $12,7,24-26$ & 104.15 & .35 & +78.5 & 3.3 & +67.2 & 223.8 & $\mathrm{~N}$ & 4.4 \\
\hline $13,1,20-22$ & 104.41 & 1.92 & -67.4 & 92.3 & & 96.2 & & \\
\hline $13,1,50-52$ & 104.71 & 1.16 & +35.4 & 16.1 & +65.7 & 43.1 & $\mathrm{~N}$ & 7.7 \\
\hline $13,1,80-82$ & 105.01 & 1.74 & +48.9 & 175.4 & +81.0 & 44.4 & $\mathrm{~N}$ & 3.3 \\
\hline $13,1,110-112$ & 105.31 & .20 & +62.9 & 40.5 & -76.9 & 166.8 & $\mathrm{R}$ & 3.2 \\
\hline $13,1,144-146$ & 105.65 & .24 & -73.4 & 345.5 & -73.1 & 177.3 & $\mathrm{R}$ & 3.4 \\
\hline $13,2,20-22$ & 105.91 & .31 & -19.4 & 193.6 & -73.4 & 203.5 & $\mathrm{R}$ & \\
\hline $13,2,50-52$ & 106.21 & .36 & +69.1 & 41.7 & -86.9 & 180.4 & $\mathrm{R}$ & 3.8 \\
\hline $13,2,80-82$ & 106.51 & .32 & +66.2 & 65.2 & +33.5 & 75.4 & $\mathrm{~N}$ & 4.1 \\
\hline $13,2,110-112$ & 106.81 & 1.28 & +48.8 & 356.5 & +71.5 & 9.2 & $\mathrm{~N}$ & 6.1 \\
\hline $13,2,140-142$ & 107.11 & .36 & +68.9 & 35.5 & +68.1 & 22.5 & $\mathrm{~N}$ & 9.6 \\
\hline $13,3,20-22$ & 107.41 & .43 & +75.3 & 352.8 & +83.6 & 31.5 & $\mathrm{~N}$ & 9.7 \\
\hline $13,3,50-52$ & 107.71 & .27 & +51.8 & 315.0 & +70.9 & 37.5 & $\mathrm{~N}$ & 9.3 \\
\hline $13,3,80-82$ & 108.01 & .62 & +76.3 & 49.6 & +71.6 & 47.8 & $N$ & 7.9 \\
\hline $13,3,110-112$ & 108.31 & .32 & -51.8 & 221.6 & +72.3 & 30.0 & $\mathrm{~N}$ & 3.7 \\
\hline $13,3,140-142$ & 108.61 & .25 & +59.3 & 56.6 & +72.4 & 22.3 & $\mathrm{~N}$ & 21.4 \\
\hline
\end{tabular}


Table A1 (continued).

\begin{tabular}{|c|c|c|c|c|c|c|c|c|}
\hline $\begin{array}{l}\text { Core, Section, } \\
\text { Interval (cm) }\end{array}$ & $\begin{array}{l}\text { Depth } \\
\text { (mbsf) }\end{array}$ & $\begin{array}{c}\mathrm{J} \\
\mathrm{NRM} \\
(\mathrm{mA} / \mathrm{m})\end{array}$ & $\begin{array}{l}\text { Incl. } \\
\text { NRM } \\
\text { (deg) }\end{array}$ & $\begin{array}{l}\text { Decl. } \\
\text { NRM } \\
\text { (deg) }\end{array}$ & $\begin{array}{c}\text { Incl } \\
\text { stable } \\
\text { (deg) }\end{array}$ & $\begin{array}{l}\text { Decl. } \\
\text { stable } \\
\text { (deg) }\end{array}$ & Pol. & $\begin{array}{l}\text { MDF } \\
(\mathrm{mT})\end{array}$ \\
\hline $13,4,20-22$ & 108.91 & .39 & +62.5 & 24.3 & +65.4 & 19.3 & $\mathrm{~N}$ & 7.6 \\
\hline $13,4,50-52$ & 109.21 & .75 & +82.5 & 103.7 & +64.2 & 24.2 & $\mathrm{~N}$ & 28.2 \\
\hline $13,4,80-82$ & 109.51 & .30 & +80.7 & 41.0 & +85.8 & 25.8 & $\mathrm{~N}$ & 18.4 \\
\hline $13,4,110-112$ & 109.81 & .54 & +51.6 & 314.7 & +78.7 & 47.9 & $\mathrm{~N}$ & 5.2 \\
\hline $13,5,20-22$ & 110.41 & .68 & +46.7 & 279.3 & +71.0 & 33.2 & $\mathrm{~N}$ & 9.6 \\
\hline $13,5,55-57$ & 110.76 & .39 & +56.5 & 248.9 & +77.7 & 45.3 & $\mathrm{~N}$ & 4.6 \\
\hline $13,5,80-82$ & 111.01 & .35 & +74.8 & 45.0 & +74.8 & 35.2 & $\mathrm{~N}$ & 4.8 \\
\hline $13,5,110-112$ & 111.31 & .19 & +36.2 & 172.4 & +76.6 & 18.1 & $\mathrm{~N}$ & 24.5 \\
\hline $13,5,140-142$ & 111.61 & .18 & +60.6 & 133.5 & +77.3 & 36.8 & $\mathrm{~N}$ & 30.7 \\
\hline $13,6,18-20$ & 111.89 & .23 & +27.9 & 329.5 & +79.2 & 46.6 & $\mathrm{~N}$ & 37.1 \\
\hline $13,6,50-52$ & 112.21 & .53 & +47.1 & 328.6 & +68.8 & 33.5 & $\mathrm{~N}$ & 4.8 \\
\hline $13,6,80-82$ & 112.51 & .28 & +75.2 & 64.4 & +69.1 & 54.0 & $\mathrm{~N}$ & 15.4 \\
\hline $13,6,110-112$ & 112.81 & .27 & +77.5 & 69.6 & +69.6 & 51.0 & $\mathrm{~N}$ & 9.2 \\
\hline $13,6,140-142$ & 113.11 & .51 & +54.4 & 60.0 & +7 & 31.3 & $\mathrm{~N}$ & 4.8 \\
\hline $13,7,31-33$ & 113.52 & .47 & -81.7 & 120.6 & -63.2 & 201.9 & $\mathrm{R}$ & 3.7 \\
\hline $15,1,42-44$ & 93 & .09 & +82.3 & 33 & +6 & & $\mathrm{~N}$ & 3.3 \\
\hline $15,1,80-82$ & 124 & .06 & +52.6 & 97.3 & & 60.0 & $\mathrm{~N}$ & \\
\hline $15,1,110-112$ & 124.61 & .40 & +49.9 & 58.0 & +6 & & $\mathrm{~N}$ & 16.5 \\
\hline $15,2,18-20$ & 125.19 & .45 & +68.4 & 191.1 & +7 & 23.3 & $\mathrm{~N}$ & 3.9 \\
\hline $15,2,48-50$ & 125 & .07 & +4 & & & 96.3 & & 11.7 \\
\hline $15,2,109-111$ & 126.10 & .35 & +35.6 & 10 & & 36.9 & $\mathrm{~N}$ & 4.2 \\
\hline $15,3,18-20$ & 126 & .43 & + & 88 & +7 & 13 & $\mathrm{~N}$ & 4.3 \\
\hline $15,3,80-82$ & 127.31 & .10 & +50.4 & 16.3 & +60.3 & 324.7 & $\mathrm{~N}$ & 9.2 \\
\hline $16,1,20-22$ & & .37 & 7 & & & & $\mathrm{~N}$ & 5.6 \\
\hline $16,1,50-52$ & 1 & .32 & + & 11 & 5 & 358 & $N$ & 14.6 \\
\hline $16,1,80-82$ & .91 & .33 & $+\varepsilon$ & 346.5 & 3 & 33.2 & $\mathrm{~N}$ & 3.7 \\
\hline $16,1,110-112$ & 129.21 & .27 & + & 0 & 2 & 5 & $\mathrm{~N}$ & 10.9 \\
\hline $16,1,140-142$ & 129.51 & .48 & + & 13 & 6 & 8.3 & $\mathrm{~N}$ & 16.2 \\
\hline $16,2,20-22$ & 129.81 & 3.50 & .4 & 2 & +6 & 27.3 & $\mathrm{~N}$ & 26.9 \\
\hline $16,2,50-52$ & 130.11 & .61 & +7 & 356.3 & $+\varepsilon$ & 355.5 & $\mathrm{~N}$ & 27.9 \\
\hline $16,2,80-82$ & 130 & .17 & -82.5 & 29 & -7 & 2 & $\mathrm{R}$ & 20.3 \\
\hline $16,2,112-114$ & 130.73 & 2.26 & -7 & & -7 & 23 & $\mathrm{R}$ & 39.7 \\
\hline $16,2,140-142$ & 131.01 & 1.21 & +75.5 & 356.3 & +8 & 345.5 & $\mathrm{~N}$ & 35.1 \\
\hline $16,3,18-20$ & 131.29 & .74 & +76.4 & 45.3 & $+\varepsilon$ & 50.7 & $\mathrm{~N}$ & \\
\hline $16,4,20-22$ & 132.81 & .3 & +52.8 & 314.7 & +6 & 327.0 & $\mathrm{~N}$ & 15.9 \\
\hline $16,4,50-52$ & 133.11 & 2.45 & +84.7 & 15 & $+\varepsilon$ & 328.4 & $\mathrm{~N}$ & 33.1 \\
\hline $16,4,80-82$ & 133 & .65 & +4 & 324.2 & & 31 & $\mathrm{~N}$ & 18.7 \\
\hline $16,4,108-110$ & 133.69 & 1.27 & +84.3 & & & 29 & $\mathrm{~N}$ & 8.0 \\
\hline $16,4,142-144$ & 134.03 & .28 & +83.3 & 340.9 & .7 & 306.7 & $\mathrm{~N}$ & 13.4 \\
\hline $16,5,19-21$ & 134.30 & .97 & +84.3 & 89.5 & .5 & 297.9 & $\mathrm{~N}$ & 29.8 \\
\hline $16,5,50-52$ & 134.61 & .55 & +94.1 & 85.3 & +71.8 & 303.0 & $\mathrm{~N}$ & 14.6 \\
\hline $16,5,80-82$ & 134.91 & .25 & +72.2 & 87.5 & +83.3 & 282.7 & $\mathrm{~N}$ & 16.2 \\
\hline $16,5,110-112$ & 135.21 & 16 & +79.5 & 120.6 & +71.2 & 259.9 & $\mathrm{~N}$ & 9.9 \\
\hline $16,5,141-143$ & 135.52 & .12 & +62.4 & 278.4 & +64.4 & 276.6 & $\mathrm{~N}$ & 5.8 \\
\hline $16,6,20-22$ & 135.81 & .17 & +89.2 & 355.4 & +78.3 & 282.4 & $\mathrm{~N}$ & 15.2 \\
\hline $16,6,49-51$ & 136.10 & .63 & +75.9 & 118.6 & +81.5 & 260.7 & $\mathrm{~N}$ & 24.1 \\
\hline $16,6,81-83$ & 136.42 & .52 & +76.9 & 261.4 & +69.3 & 267.1 & $\mathrm{~N}$ & 12.9 \\
\hline $16,6,111-113$ & 136.72 & .16 & +67.7 & 80.8 & +71.7 & 36.1 & N & 9.5 \\
\hline
\end{tabular}




\begin{tabular}{|c|c|c|c|c|c|c|c|c|}
\hline $\begin{array}{l}\text { Core, Section, } \\
\text { Interval }(\mathrm{cm})\end{array}$ & $\begin{array}{l}\text { Depth } \\
\text { (mbsf) }\end{array}$ & $\begin{array}{c}\mathrm{J} \\
\mathrm{NRM} \\
(\mathrm{mA} / \mathrm{m})\end{array}$ & $\begin{array}{l}\text { Incl. } \\
\text { NRM } \\
\text { (deg) }\end{array}$ & $\begin{array}{l}\text { Decl. } \\
\text { NRM } \\
\text { (deg) }\end{array}$ & $\begin{array}{c}\text { Incl } \\
\text { stable } \\
\text { (deg) }\end{array}$ & $\begin{array}{l}\text { Decl. } \\
\text { stable } \\
\text { (deg) }\end{array}$ & Pol. & $\begin{array}{l}\text { MDF } \\
(\mathrm{mT})\end{array}$ \\
\hline $16,7,19-21$ & 137.30 & .13 & +73.8 & 74.3 & +71.4 & 26.4 & $\mathrm{~N}$ & 4.9 \\
\hline $16,7,50-52$ & 137.61 & .07 & +77.5 & 271.6 & +67.2 & 29.4 & $\mathrm{~N}$ & \\
\hline $16,7,80-82$ & 137.91 & .17 & +67.4 & 43.0 & +71.5 & 27.7 & $\mathrm{~N}$ & 4.0 \\
\hline $16,7,110-112$ & 138.21 & .66 & +82.7 & 33.0 & +70.1 & 38.8 & $\mathrm{~N}$ & 4.5 \\
\hline $17,1,50-52$ & 138.51 & .29 & +72.1 & 65.8 & +62.9 & 38.9 & $\mathrm{~N}$ & 4.6 \\
\hline $17,1,80-82$ & 138.81 & .21 & +73.8 & 74.5 & +66.9 & 51.3 & $\mathrm{~N}$ & 5.0 \\
\hline $17,1,110-112$ & 139.11 & .65 & +65.9 & 67.6 & +70.2 & 50.4 & $\mathrm{~N}$ & 23.4 \\
\hline $17,1,140-142$ & 139.41 & .47 & +51.3 & 59.8 & +72.5 & 52.7 & $\mathrm{~N}$ & 5.0 \\
\hline $17,2,20-22$ & 139.71 & .25 & +61.8 & 60.4 & +68.0 & 49.0 & $\mathrm{~N}$ & 5.1 \\
\hline $17,2,50-52$ & 140.01 & .31 & +82.1 & 40.2 & +74.2 & 55.3 & $\mathrm{~N}$ & 3.8 \\
\hline $17,2,80-82$ & 140.31 & .47 & +81.2 & 26.1 & +63.3 & 57.6 & $\mathrm{~N}$ & 4.4 \\
\hline $17,2,110-112$ & 140.61 & .15 & +76.7 & 98.5 & +68.4 & 72.1 & $\mathrm{~N}$ & 3.5 \\
\hline $17,2,140-142$ & 140.91 & .22 & +82.2 & 54.4 & +67.1 & 58.5 & $\mathrm{~N}$ & 3.9 \\
\hline $17,3,20-22$ & 141.21 & .17 & +82.4 & 98.7 & +69.9 & 64.9 & $\mathrm{~N}$ & 3.4 \\
\hline $17,3,50-52$ & 141.51 & .13 & +77.2 & 76.4 & +78.2 & 89.3 & $\mathrm{~N}$ & 3.2 \\
\hline $17,3,80-82$ & 141.81 & .14 & +75.6 & 343.2 & +69.3 & 55.8 & $\mathrm{~N}$ & 4.9 \\
\hline $17,3,110-112$ & 142.11 & .12 & +75.6 & 38.1 & +71.8 & 35.7 & $\mathrm{~N}$ & 3.4 \\
\hline $17,4,20-22$ & 142.71 & .16 & +84.2 & 95.2 & +73.3 & 40.1 & $\mathrm{~N}$ & 4.4 \\
\hline $17,4,50-52$ & 143.01 & .06 & +63.3 & 313.3 & -69.2 & 273.7 & $\mathrm{R}$ & 4.1 \\
\hline $17,4,80-82$ & 143.31 & .09 & -69.9 & 157.7 & -70.2 & 189.0 & $\mathrm{R}$ & 6.0 \\
\hline $17,4,110-112$ & 143.61 & .06 & -60.3 & 191.6 & -64.1 & 189.5 & $\mathrm{R}$ & 13.8 \\
\hline $17,4,140-142$ & 143.91 & .11 & +75.6 & 357.1 & -68.0 & 226.7 & $\mathrm{R}$ & 3.9 \\
\hline $17,5,20-22$ & 144.21 & .14 & +71.0 & 85.9 & +71.5 & 63.4 & $\mathrm{~N}$ & 3.6 \\
\hline $17,5,48-50$ & 144.49 & .18 & +68.3 & 73.4 & +61.9 & 80.6 & $\mathrm{~N}$ & 4.4 \\
\hline $17,5,48-50$ & 144.49 & .18 & +68.3 & 73.4 & +67.1 & 87.4 & $\mathrm{~N}$ & 4.4 \\
\hline $17,5,80-82$ & 144.81 & .38 & +72.7 & 82.3 & +75.5 & 114.0 & $\mathrm{~N}$ & 4.4 \\
\hline $17,6,20-22$ & 145.71 & .17 & +75.3 & 99.9 & +66.3 & 97.1 & $\mathrm{~N}$ & 4.0 \\
\hline $17,6,50-52$ & 146.01 & .14 & +72.6 & 140.2 & +75.3 & 90.9 & $\mathrm{~N}$ & 4.2 \\
\hline $17,6,80-82$ & 146.31 & .23 & +85.3 & .3 & +76.9 & 95.0 & $\mathrm{~N}$ & 4.3 \\
\hline $17,6,110-112$ & 146.61 & .16 & +77.0 & 67.0 & +84.8 & 73.3 & $\mathrm{~N}$ & 4.0 \\
\hline $17,6,140-142$ & 146.91 & .27 & +82.0 & 72.6 & +74.7 & 74.8 & $\mathrm{~N}$ & 4.3 \\
\hline $17,7,20-22$ & 147.21 & .35 & +75.9 & 61.8 & +75.9 & 70.2 & $\mathrm{~N}$ & 4.4 \\
\hline $18,1,20-22$ & 148.01 & .09 & +57.2 & 169.0 & +71.4 & 13 & $\mathrm{~N}$ & 11.8 \\
\hline $18,1,50-52$ & 148.31 & .08 & +78.2 & 114.1 & +66.8 & 105.9 & $\mathrm{~N}$ & 9.1 \\
\hline $18,1,80-82$ & 148.61 & .14 & +76.8 & 50.7 & +76.8 & 101.8 & $\mathrm{~N}$ & 6.0 \\
\hline $18,1,110-112$ & 148.91 & .25 & +47.7 & 117.9 & +67.6 & 109.0 & $\mathrm{~N}$ & 5.6 \\
\hline $18,1,140-142$ & 149.21 & .39 & +54.5 & 90.6 & +65.7 & 114.0 & $\mathrm{~N}$ & 3.7 \\
\hline $18,2,20-22$ & 149.51 & .23 & +54.6 & 76.6 & +69.2 & 97.8 & $\mathrm{~N}$ & 6.9 \\
\hline $18,2,50-52$ & 149.81 & .10 & +75.0 & 127.6 & +67.2 & 112.6 & $\mathrm{~N}$ & \\
\hline $18,2,80-82$ & 150.11 & .07 & +78.5 & 22.8 & +77.0 & 110.2 & $\mathrm{~N}$ & 3.6 \\
\hline $18,2,110-112$ & 150.41 & .09 & +50.5 & 99.5 & +78.0 & 115.9 & $\mathrm{~N}$ & \\
\hline $18,2,140-142$ & 150.71 & .18 & +12.9 & 132.6 & -66.3 & 246.3 & & 3.2 \\
\hline $18,3,20-22$ & 151.01 & .67 & +8.7 & 115.1 & +65.3 & 87.6 & $\mathrm{~N}$ & 4.2 \\
\hline $18,3,50-52$ & 151.31 & 1.70 & +82.3 & 101.5 & +83.3 & 95.5 & $\mathrm{~N}$ & 6.1 \\
\hline $18,3,80-82$ & 151.61 & 1.10 & +76.7 & 81.1 & +73.3 & 87.4 & $\mathrm{~N}$ & 10.2 \\
\hline $18,3,110-112$ & 151.91 & .10 & +65.3 & 81.2 & +81.7 & 79.8 & $\mathrm{~N}$ & 10.4 \\
\hline $18,3,140-142$ & 152.21 & 4.92 & +80.4 & 33.3 & +82.5 & 77.5 & $\mathrm{~N}$ & 26.1 \\
\hline $18,4,20-22$ & 152.51 & .04 & +20.6 & 159.8 & -58.2 & 341.5 & & \\
\hline
\end{tabular}


Table A1 (continued).

\begin{tabular}{|c|c|c|c|c|c|c|c|c|}
\hline $\begin{array}{l}\text { Core, Section, } \\
\text { Interval (cm) }\end{array}$ & $\begin{array}{l}\text { Depth } \\
\text { (mbsf) }\end{array}$ & $\begin{array}{c}\mathrm{J} \\
\mathrm{NRM} \\
(\mathrm{mA} / \mathrm{m})\end{array}$ & $\begin{array}{l}\text { Incl. } \\
\text { NRM } \\
\text { (deg) }\end{array}$ & $\begin{array}{l}\text { Decl. } \\
\text { NRM } \\
\text { (deg) }\end{array}$ & $\begin{array}{c}\text { Incl } \\
\text { stable } \\
\text { (deg) }\end{array}$ & $\begin{array}{l}\text { Decl. } \\
\text { stable } \\
\text { (deg) }\end{array}$ & Pol. & $\begin{array}{l}\mathrm{MDF} \\
(\mathrm{mT})\end{array}$ \\
\hline $18,4,50-52$ & 152.81 & .10 & +59.7 & 102.6 & +65.6 & 106.1 & $\mathrm{~N}$ & 6.8 \\
\hline $18,4,80-82$ & 153.11 & .15 & +75.6 & 72.0 & +77.2 & 108.9 & $\mathrm{~N}$ & 23.5 \\
\hline $18,4,110-112$ & 153.41 & .09 & +73.2 & 93.7 & +74.0 & 104.5 & $\mathrm{~N}$ & 12.9 \\
\hline $18,4,140-142$ & 153.71 & .22 & +67.7 & 108.5 & +75.9 & 118.6 & $\mathrm{~N}$ & 11.8 \\
\hline $18,5,20-22$ & 154.01 & .10 & -64.7 & 280.4 & +70.5 & 89.6 & $\mathrm{~N}$ & 3.1 \\
\hline $18,5,50-52$ & 154.31 & .05 & +65.3 & 32.5 & +65.3 & 102.8 & $\mathrm{~N}$ & 6.8 \\
\hline $18,5,80-82$ & 154.61 & .04 & +79.6 & 145.9 & +70.8 & 113.5 & $\mathrm{~N}$ & 5.3 \\
\hline $18,5,110-112$ & 154.91 & .25 & +60.9 & 131.4 & +69.3 & 119.6 & $\mathrm{~N}$ & 14.6 \\
\hline $18,6,20-22$ & 155.51 & .14 & +64.6 & 86.1 & +67.9 & 111.5 & $\mathrm{~N}$ & 6.2 \\
\hline $18,6,50-52$ & 155.81 & .19 & +77.9 & 29.8 & +67.0 & 101.7 & $\mathrm{~N}$ & 3.7 \\
\hline $18,6,80-82$ & 156.11 & .10 & +69.4 & 125.2 & +72.3 & 115.8 & $\mathrm{~N}$ & 7.5 \\
\hline $18,6,110-112$ & 156.41 & .11 & +74.7 & 120.0 & +61.5 & 102.4 & $\mathrm{~N}$ & 4.2 \\
\hline $18,6,140-142$ & 156.71 & .09 & +76.2 & 97.9 & +75.2 & 108.3 & $\mathrm{~N}$ & 9.3 \\
\hline $18,7,20-22$ & 157.01 & .06 & +68.5 & 83.6 & +75.7 & 86.5 & $\mathrm{~N}$ & 5.9 \\
\hline $19,1,80-82$ & 158 & .1 & +84 & 281 & +65 & & $\mathrm{~N}$ & 5.0 \\
\hline $19,1,110-112$ & 158.81 & .1 & +83.6 & 109.6 & +67.1 & 10.0 & $\mathrm{~N}$ & 3.4 \\
\hline $19,1,140-142$ & 159.11 & .3 & +76.2 & 49.4 & +66.0 & 35.1 & $\mathrm{~N}$ & 4.4 \\
\hline $19,2,20-22$ & 159.41 & .1 & +80.0 & 35.8 & +70.7 & 28.7 & $\mathrm{~N}$ & 3.8 \\
\hline $19,2,50-52$ & 159.71 & .2 & +81.7 & 45.8 & +75.1 & 50.6 & $\mathrm{~N}$ & 8.8 \\
\hline $19,2,86-88$ & 160.07 & .2 & +75.3 & 150.4 & +65.9 & 52.1 & $\mathrm{~N}$ & 4.8 \\
\hline $19,2,110-112$ & 160 & .4 & +75.5 & $4 \xi$ & +6 & 39.6 & $\mathrm{~N}$ & 9.4 \\
\hline $19,2,140-142$ & 160.61 & .2 & +73 & 98 & +6 & 59.0 & $\mathrm{~N}$ & 4.6 \\
\hline $19,3,20-22$ & 160.91 & .31 & +78.4 & 66.6 & +80.4 & 39.4 & $\mathrm{~N}$ & 4.6 \\
\hline $19,3,50-52$ & 161.21 & .23 & +81.5 & 19.3 & +81.0 & 27.6 & $\mathrm{~N}$ & 6.0 \\
\hline $19,3,80-82$ & 161.51 & .19 & +87.8 & 90.2 & +79.3 & 31.4 & $\mathrm{~N}$ & 4.5 \\
\hline $19,3,110-112$ & 161.81 & .17 & +85.7 & 51.2 & +85.5 & 14.1 & $\mathrm{~N}$ & 4.0 \\
\hline $19,3,140-142$ & 162.11 & .3 & +85.4 & 51.3 & +82.8 & 15.2 & $\mathrm{~N}$ & 8.7 \\
\hline $19,4,20-22$ & 162.41 & .18 & +85.0 & 77.5 & +80.6 & 33.0 & $\mathrm{~N}$ & 5.2 \\
\hline $19,4,50-52$ & 162.71 & .20 & +84.8 & 63.8 & +83.4 & 29.9 & $\mathrm{~N}$ & 4.6 \\
\hline $19,4,80-82$ & 163.01 & .26 & +81.1 & 48.1 & +76.0 & 31.0 & $\mathrm{~N}$ & 4.9 \\
\hline $19,4,110-112$ & 163.31 & .2 & +87.2 & 358.6 & +74.0 & 24.8 & $\mathrm{~N}$ & 13.0 \\
\hline $19,5,20-22$ & 163.91 & .77 & +80.4 & 11.2 & +76.0 & 15.0 & $\mathrm{~N}$ & 3.8 \\
\hline $19,5,50-52$ & 164.21 & .28 & +80.1 & 181.8 & +71.3 & 31.4 & $\mathrm{~N}$ & 5.4 \\
\hline $19,5,80-82$ & 164.51 & .28 & +26.4 & 346.7 & +61.6 & 20.2 & $\mathrm{~N}$ & 2.9 \\
\hline $19,5,110-112$ & 164.81 & .25 & +73.9 & 29.8 & +79.2 & 27.0 & $\mathrm{~N}$ & 3.7 \\
\hline $19,5,140-142$ & 165.11 & 1.19 & -18.8 & 272.8 & -43.2 & 247.0 & & 5.0 \\
\hline $19,6,20-22$ & 165.41 & .17 & +77.1 & 26.1 & +72.6 & 18.5 & $\mathrm{~N}$ & 3.5 \\
\hline $19,6,50-52$ & 165.71 & .18 & +85.9 & 28.8 & +80.1 & 37.4 & $\mathrm{~N}$ & 3.5 \\
\hline $19,6,80-82$ & 166.01 & .30 & +78.0 & 34.4 & +76.5 & 50.1 & $\mathrm{~N}$ & 3.9 \\
\hline $19,6,110-112$ & 166.31 & .22 & +28.5 & 340.5 & +84.1 & 52.6 & $\mathrm{~N}$ & 4.4 \\
\hline $19,6,140-142$ & 166.61 & .11 & +64.5 & 146.7 & +85.9 & 46.2 & $\mathrm{~N}$ & 7.0 \\
\hline $19,7,20-22$ & 166.91 & .38 & -39.2 & 32.1 & +78.1 & 52.2 & $\mathrm{~N}$ & 3.7 \\
\hline $20,1,20-22$ & 167.61 & .1 & +71.9 & 190.0 & +63.0 & & $\mathrm{~N}$ & 4.0 \\
\hline $20,1,50-52$ & 167.91 & 4.00 & +16.3 & 227.3 & +67.8 & 217.1 & $\mathrm{~N}$ & 4.9 \\
\hline $20,1,80-82$ & 168.21 & .18 & +65.2 & 84.2 & +83.7 & 205.0 & $\mathrm{~N}$ & 9.4 \\
\hline $20,1,110-112$ & 168.51 & .28 & +75.0 & 184.3 & +63.8 & 202.0 & $\mathrm{~N}$ & 3.9 \\
\hline $20,1,140-142$ & 168.81 & .20 & +19.5 & 299.4 & +78.9 & 226.4 & $\mathrm{~N}$ & 4.3 \\
\hline $20,2,20-22$ & 169.11 & .55 & +75.9 & 148.1 & +73.3 & 198.0 & $\mathrm{~N}$ & 6.2 \\
\hline
\end{tabular}


Table A1 (continued).

\begin{tabular}{|c|c|c|c|c|c|c|c|c|}
\hline $\begin{array}{l}\text { Core, Section, } \\
\text { Interval }(\mathrm{cm})\end{array}$ & $\begin{array}{l}\text { Depth } \\
\text { (mbsf) }\end{array}$ & $\underset{(\mathrm{mA} / \mathrm{m})}{\stackrel{\mathrm{J}}{\mathrm{NRM}}}$ & $\begin{array}{l}\text { Incl. } \\
\text { NRM } \\
\text { (deg) }\end{array}$ & $\begin{array}{l}\text { Decl. } \\
\text { NRM } \\
\text { (deg) }\end{array}$ & $\begin{array}{c}\text { Incl } \\
\text { stable } \\
\text { (deg) }\end{array}$ & $\begin{array}{l}\text { Decl. } \\
\text { stable } \\
\text { (deg) }\end{array}$ & Pol. & $\begin{array}{l}\text { MDF } \\
(\mathrm{mT})\end{array}$ \\
\hline $20,2,50-52$ & 169.41 & .37 & +88.4 & 106.1 & +83.9 & 215.7 & $\mathrm{~N}$ & 3.7 \\
\hline $20,2,80-82$ & 169.71 & .16 & +83.2 & 200.1 & +79.7 & 203.7 & $\mathrm{~N}$ & 4.9 \\
\hline $20,2,110-112$ & 170.01 & .78 & +80.6 & 229.9 & +77.8 & 227.4 & $\mathrm{~N}$ & 35.0 \\
\hline $20,2,140-142$ & 170.31 & .27 & +67.7 & 16.8 & +79.7 & 232.1 & $\mathrm{~N}$ & 16.9 \\
\hline $20,3,20-22$ & 170.61 & .07 & +82.5 & 220.5 & +64.8 & 202.7 & $\mathrm{~N}$ & 9.8 \\
\hline $20,3,50-52$ & 170.91 & .09 & +57.9 & 120.6 & +63.9 & 191.5 & $\mathrm{~N}$ & 19.3 \\
\hline $20,3,80-82$ & 171.21 & .77 & +61.0 & 204.5 & +83.5 & 207.8 & $\mathrm{~N}$ & 46.0 \\
\hline $20,3,110-112$ & 171.51 & .15 & +75.0 & 163.4 & +64.8 & 225.1 & $\mathrm{~N}$ & 44.0 \\
\hline $20,3,140-142$ & 171.81 & .17 & +66.2 & 124.9 & +72.8 & 233.5 & $\mathrm{~N}$ & 8.2 \\
\hline $20,4,20-22$ & 172.11 & .61 & +33.0 & 198.4 & +76.4 & 213.4 & N & 6.6 \\
\hline $20,4,50-52$ & 172.41 & .14 & +63.0 & 92.2 & 1 & 195.5 & $\mathrm{~N}$ & 3.9 \\
\hline $20,4,80-82$ & 172.71 & .13 & +66.5 & 181.3 & 8 & 217.2 & $\mathrm{~N}$ & 21.3 \\
\hline $20,4,110-112$ & 173.01 & .21 & -66.1 & 151.6 & 2 & 20 & $\mathrm{~N}$ & 2.0 \\
\hline $20,5,20-22$ & 173.61 & .09 & +68.2 & 92.4 & 1 & 217.3 & $\mathrm{~N}$ & \\
\hline $20,5,50-52$ & 173.91 & .20 & +7 & 229.9 & & .2 & $\mathrm{~N}$ & 11.3 \\
\hline $20,5,80-82$ & 174.21 & .28 & +85.7 & 286.4 & +77.1 & 225.8 & $\mathrm{~N}$ & 9.4 \\
\hline $20,5,110-112$ & 174.51 & 1.09 & +64.8 & 242.9 & +64.6 & 221.3 & $\mathrm{~N}$ & 3.2 \\
\hline $20,5,140-142$ & 174.81 & .78 & +58.4 & 267.2 & +79.7 & 221.9 & $\mathrm{~N}$ & 3.5 \\
\hline $20,6,20-22$ & 175.11 & .31 & +45.8 & 240.0 & +63.6 & 191.4 & $\mathrm{~N}$ & 3.1 \\
\hline $20,6,50-52$ & 175.41 & .30 & +83.1 & 126.8 & +83.6 & 186.7 & $\mathrm{~N}$ & 8.8 \\
\hline $20,6,80-82$ & 175.71 & .18 & +81.7 & 355.7 & +81.3 & 213.3 & $\mathrm{~N}$ & 12.3 \\
\hline $20,6,110-112$ & 176.01 & .17 & +80.5 & 150.5 & +70.7 & 187.8 & $\mathrm{~N}$ & 6.4 \\
\hline $20,6,140-142$ & 176.31 & .61 & +12.2 & 135.0 & +83.9 & 183.3 & $\mathrm{~N}$ & 4.0 \\
\hline $20,7,20-22$ & 176.61 & 1.32 & +87.1 & 4.9 & +86.3 & 202.0 & $\mathrm{~N}$ & 35.0 \\
\hline $20,7,52-54$ & 176.93 & .11 & +79.3 & 134.5 & +74.7 & 186.6 & $\mathrm{~N}$ & 21.7 \\
\hline $21,1,80-82$ & 177.91 & .36 & +74.3 & 352.7 & -59.5 & 162.6 & R & 12.1 \\
\hline $21,1,110-112$ & 178.21 & .07 & +57.9 & 214.5 & -65.3 & 209.5 & $\mathrm{R}$ & 4.7 \\
\hline $21,1,140-142$ & 178.51 & .05 & +51.1 & 197.0 & -69.1 & 206.1 & R & 13.8 \\
\hline $21,2,20-22$ & 178.81 & .19 & +78.2 & 191.1 & -60.2 & 195.9 & $\mathrm{R}$ & 5.0 \\
\hline $21,2,50-52$ & 179.11 & .07 & +60.5 & 167.0 & -52.6 & 171.0 & $\mathrm{R}$ & 34.2 \\
\hline $21,2,80-82$ & 179.41 & .06 & +74.2 & 150.5 & -62.8 & 186.9 & $\mathrm{R}$ & \\
\hline $21,2,110-112$ & 179.71 & .19 & +71.8 & 90.7 & -79.0 & 152.2 & $\mathrm{R}$ & 4.1 \\
\hline $21,3,20-22$ & 180.31 & .21 & +66.7 & 108.4 & +66.3 & 114.0 & $\mathrm{~N}$ & 9.5 \\
\hline $21,3,50-52$ & 180.61 & 1.64 & +77.5 & 28.3 & +73.9 & 52.1 & $\mathrm{~N}$ & 15.5 \\
\hline $21,3,80-82$ & 180.91 & 4.47 & +71.1 & 100.3 & +71.5 & 68.4 & $\mathrm{~N}$ & 4.4 \\
\hline $21,3,110-112$ & 181.21 & 1.34 & +80.3 & 57.7 & +77.1 & 59.9 & $\mathrm{~N}$ & 4.0 \\
\hline $21,4,20-22$ & 181.81 & .13 & +68.3 & 42.9 & +62.7 & 58.7 & $\mathrm{~N}$ & 5.3 \\
\hline $21,4,50-52$ & 182.11 & .17 & -10.2 & 31.2 & +75.6 & 51.8 & $\mathrm{~N}$ & 24.6 \\
\hline $21,4,80-82$ & 182.41 & .14 & +60.7 & 77.2 & +75.6 & 35.8 & $\mathrm{~N}$ & 6.1 \\
\hline $21,4,110-112$ & 182.71 & .16 & +55.4 & 139.6 & -33.8 & 126.2 & $\mathrm{R}$ & 9.0 \\
\hline $21,4,140-142$ & 183.01 & .07 & +63.8 & 170.8 & -69.1 & 172.2 & $\mathrm{R}$ & \\
\hline $21,5,20-22$ & 183.31 & .11 & +62.0 & 128.5 & -31.9 & 160.4 & $\mathrm{R}$ & 4.7 \\
\hline $21,5,50-52$ & 183.61 & .07 & +76.6 & 63.2 & +77.1 & 64.9 & $\mathrm{~N}$ & 10.7 \\
\hline $21,5,80-82$ & 183.91 & .1 & +74.7 & 111.9 & +73.0 & 68.1 & $\mathrm{~N}$ & 4.5 \\
\hline $21,5,110-112$ & 184.21 & .2 & +81.7 & 50.0 & +73.9 & 63.5 & $\mathrm{~N}$ & 4.7 \\
\hline $21,5,140-142$ & 184.51 & .47 & +85.0 & 88.3 & +79.8 & 60.9 & $\mathrm{~N}$ & 7.9 \\
\hline $21,6,20-22$ & 184.81 & .14 & +75.4 & 214.0 & +67.9 & 74.3 & $\mathrm{~N}$ & 3.8 \\
\hline $21,6,80-82$ & 185.41 & .17 & +72.3 & 76.9 & +72.3 & 71.0 & $\mathrm{~N}$ & 12.1 \\
\hline $21,6,110-112$ & 185.71 & .21 & +75.2 & 136.0 & +76.7 & 53.3 & $\mathrm{~N}$ & 14.7 \\
\hline
\end{tabular}


Table A1 (continued).

\begin{tabular}{|c|c|c|c|c|c|c|c|c|}
\hline $\begin{array}{l}\text { Core, Section, } \\
\text { Interval }(\mathrm{cm})\end{array}$ & $\begin{array}{l}\text { Depth } \\
\text { (mbsf) }\end{array}$ & $\begin{array}{c}\mathrm{J} \\
\mathrm{NRM} \\
(\mathrm{mA} / \mathrm{m})\end{array}$ & $\begin{array}{l}\text { Incl. } \\
\text { NRM } \\
\text { (deg) }\end{array}$ & $\begin{array}{l}\text { Decl. } \\
\text { NRM } \\
\text { (deg) }\end{array}$ & $\begin{array}{c}\text { Incl } \\
\text { stable } \\
\text { (deg) }\end{array}$ & $\begin{array}{l}\text { Decl. } \\
\text { stable } \\
\text { (deg) }\end{array}$ & Pol. & $\begin{array}{l}\text { MDF } \\
(\mathrm{mT})\end{array}$ \\
\hline $21,6,140-142$ & 186.01 & 2.19 & +80.9 & 48.1 & +80.3 & 60.5 & $\mathrm{~N}$ & 35.0 \\
\hline $21,7,20-22$ & 186.31 & .15 & +84.3 & 30.2 & +84.1 & 64.5 & $\mathrm{~N}$ & 5.4 \\
\hline $22,1,20-22$ & 186.91 & .23 & +72.0 & 143.7 & +76.2 & 141.6 & $\mathrm{~N}$ & 9.9 \\
\hline $22,1,50-52$ & 187.21 & .11 & +64.4 & 129.2 & +60.4 & 133.2 & $\mathrm{~N}$ & 12.1 \\
\hline $22,1,80-82$ & 187.51 & .31 & +85.6 & 109.7 & +85.6 & 132.3 & $\mathrm{~N}$ & 14.5 \\
\hline $22,1,110-112$ & 187.81 & .87 & +78.1 & 122.9 & +71.1 & 130.4 & $\mathrm{~N}$ & 13.3 \\
\hline $22,1,140-142$ & 188.11 & .89 & +72.4 & 163.8 & +66.7 & 140.8 & $\mathrm{~N}$ & 8.4 \\
\hline $22,2,20-22$ & 188.41 & .13 & +73.0 & 126.1 & +65.9 & 122.1 & $\mathrm{~N}$ & 12.2 \\
\hline $22,2,50-52$ & 188.71 & 1.46 & +74.6 & 153.1 & +68.8 & 142.6 & $\mathrm{~N}$ & 16.5 \\
\hline $22,2,80-82$ & 189.01 & .80 & +77.1 & 195.1 & +76.0 & 148.6 & $\mathrm{~N}$ & 21.7 \\
\hline $22,2,110-112$ & 189.31 & 1.05 & +71.3 & 153.7 & +74.0 & 151.4 & $\mathrm{~N}$ & 22.5 \\
\hline $22,2,140-142$ & 189.61 & .20 & +69.5 & 143.8 & +69.5 & 147.2 & $\mathrm{~N}$ & 19.1 \\
\hline $22,3,20-22$ & 189.91 & 1.27 & +87.5 & 46.8 & +84.6 & 132.9 & $\mathrm{~N}$ & 23.0 \\
\hline $22,3,50-52$ & 190.21 & .33 & +65.5 & 155.2 & +75.9 & 151.6 & $\mathrm{~N}$ & 12.8 \\
\hline $22,3,80-82$ & 190.51 & .07 & +75.5 & 143.9 & +77.0 & 140.9 & $\mathrm{~N}$ & 12.0 \\
\hline $22,3,110-112$ & 190.81 & 2.67 & +39.4 & 174.9 & +63.9 & 138.3 & $\mathrm{~N}$ & 19.0 \\
\hline $22,3,140-142$ & 191.11 & .12 & -5.6 & 33.4 & +66.6 & 150.5 & $\mathrm{~N}$ & 2.3 \\
\hline $22,4,20-22$ & 191.41 & .10 & +48.9 & 166.2 & +66.1 & 139.8 & $\mathrm{~N}$ & 5.0 \\
\hline $22,4,50-52$ & 191.71 & .28 & -20.8 & 210.9 & -69.8 & 287.9 & $\mathrm{R}$ & 4.6 \\
\hline $22,4,80-82$ & 192.01 & .06 & +63.6 & 29.6 & -76.0 & 296.2 & $\mathrm{R}$ & 4.7 \\
\hline $22,4,110-112$ & 192.31 & .12 & +77.4 & 253.4 & +77.4 & 358.1 & & 3.9 \\
\hline $22,5,20-22$ & 192.91 & .07 & +28.8 & 285.7 & -61.0 & 207.3 & $\mathrm{R}$ & 2.1 \\
\hline $22,5,50-52$ & 193.21 & .19 & -76.5 & 203.8 & -78.1 & 179.5 & $\mathrm{R}$ & 4.5 \\
\hline $22,5,80-82$ & 193.51 & .19 & -14.6 & 199.1 & -63.1 & 208.1 & $\mathrm{R}$ & 2.9 \\
\hline $22,5,110-112$ & 193.81 & .08 & +73.7 & 213.3 & -71.1 & 179.8 & $R$ & 29.1 \\
\hline $22,5,140-142$ & 194.11 & .04 & +77.4 & 128.1 & -58.7 & 186.8 & $\mathrm{R}$ & 1.9 \\
\hline $22,6,20-22$ & 194.41 & .04 & +60.1 & 2.0 & -77.2 & 190.3 & $\mathrm{R}$ & 4.1 \\
\hline $22,6,50-52$ & 194.71 & .17 & +77.2 & 164.3 & -60.6 & 178.8 & $\mathrm{R}$ & 1.8 \\
\hline $22,6,80-82$ & 195.01 & .05 & +67.7 & 146.7 & -65.8 & 170.3 & $\mathrm{R}$ & 2.5 \\
\hline $22,6,110-112$ & 195.31 & .16 & +67.6 & 166.5 & -61.7 & 192.5 & $\mathrm{R}$ & 4.0 \\
\hline $22,6,140-142$ & 195.61 & .10 & +56.7 & 172.3 & -76.8 & 216.3 & $\mathrm{R}$ & \\
\hline $23,1,11-13$ & 196.12 & .70 & -52.7 & 104.2 & & & $\mathrm{R}$ & 4.2 \\
\hline $23,1,40-42$ & 196.41 & .67 & +68.5 & 162.6 & +70.7 & 170.9 & $\mathrm{~N}$ & 27.6 \\
\hline $23,1,70-72$ & 196.71 & .18 & +74.2 & 27.4 & +67.7 & 130.8 & $\mathrm{~N}$ & 4.8 \\
\hline $23,1,100-102$ & 197.01 & .34 & +82.9 & 110.7 & +74.3 & 111.9 & $\mathrm{~N}$ & 6.9 \\
\hline $23,1,130-132$ & 197.31 & .26 & +82.6 & 129.5 & +76.4 & 113.3 & $\mathrm{~N}$ & 12.0 \\
\hline $23,2,10-12$ & 197.61 & .31 & +70.8 & 93.1 & +68.2 & 96.5 & $\mathrm{~N}$ & 39.4 \\
\hline $23,2,40-42$ & 197.91 & .21 & +80.7 & 137.6 & +76.7 & 113.4 & $\mathrm{~N}$ & 8.9 \\
\hline $23,2,70-72$ & 198.21 & .20 & +53.0 & 264.5 & +70.8 & 125.9 & $\mathrm{~N}$ & 3.6 \\
\hline $23,2,100-102$ & 198.51 & .32 & +57.0 & 164.0 & +69.7 & 131.3 & $\mathrm{~N}$ & 4.5 \\
\hline $23,2,130-132$ & 198.81 & .22 & +71.3 & 151.0 & +75.1 & 130.1 & $\mathrm{~N}$ & 13.8 \\
\hline $23,3,10-12$ & 199.1 & .4 & +41.4 & 135.0 & +69.9 & 131.8 & $\mathrm{~N}$ & 3.8 \\
\hline $23,3,40-42$ & 199.41 & .2 & +64.3 & 91.9 & +85.3 & 127.8 & $\mathrm{~N}$ & 3.9 \\
\hline $23,3,70-72$ & 199.71 & .39 & +35.8 & 23.0 & +60.7 & 125.9 & $\mathrm{~N}$ & 3.6 \\
\hline $23,3,100-102$ & 200.01 & .49 & -8.4 & 265.4 & +84.5 & 157.2 & $\mathrm{~N}$ & 3.8 \\
\hline $23,3,130-132$ & 200.31 & .15 & +87.2 & 219.3 & +60.3 & 129.1 & $\mathrm{~N}$ & 13.3 \\
\hline $23,4,9-11$ & 200.60 & .23 & +87.0 & 31.3 & +75.6 & 132.6 & $\mathrm{~N}$ & 12.3 \\
\hline $23,4,39-41$ & 200.90 & .28 & +77.1 & 132.2 & +75.9 & 137.4 & $\mathrm{~N}$ & 16.4 \\
\hline
\end{tabular}


Table A1 (continued).

\begin{tabular}{|c|c|c|c|c|c|c|c|c|}
\hline $\begin{array}{l}\text { Core, Section, } \\
\text { Interval }(\mathrm{cm})\end{array}$ & $\begin{array}{l}\text { Depth } \\
\text { (mbsf) }\end{array}$ & $\underset{(\mathrm{mA} / \mathrm{m})}{\mathrm{J}}$ & $\begin{array}{l}\text { Incl. } \\
\text { NRM } \\
\text { (deg) }\end{array}$ & $\begin{array}{l}\text { Decl. } \\
\text { NRM } \\
\text { (deg) }\end{array}$ & $\begin{array}{c}\text { Incl } \\
\text { stable } \\
\text { (deg) }\end{array}$ & $\begin{array}{l}\text { Decl. } \\
\text { stable } \\
\text { (deg) }\end{array}$ & Pol. & $\begin{array}{l}\mathrm{MDF} \\
(\mathrm{mT})\end{array}$ \\
\hline $23,4,69-71$ & 201.20 & .24 & +72.9 & 111.7 & +72.7 & 137.2 & $\mathrm{~N}$ & 17.1 \\
\hline $23,4,101-103$ & 201.52 & .16 & +20.3 & 96.9 & +65.3 & 127.8 & $\mathrm{~N}$ & 3.5 \\
\hline $23,4,130-132$ & 201.81 & .15 & +49.6 & 281.1 & +61.0 & 127.3 & $\mathrm{~N}$ & 4.9 \\
\hline $23,5,9-11$ & 202.10 & .23 & +73.3 & 157.2 & +72.2 & 130.2 & $\mathrm{~N}$ & 9.9 \\
\hline $23,5,39-41$ & 202.40 & .11 & +67.7 & 90.5 & +60.9 & 114.8 & $\mathrm{~N}$ & 5.0 \\
\hline $23,5,69-71$ & 202.70 & .20 & +87.8 & 135.9 & +79.9 & 115.4 & $\mathrm{~N}$ & 9.4 \\
\hline $23,5,101-103$ & 203.02 & .10 & +82.6 & 110.4 & +72.1 & 125.3 & $\mathrm{~N}$ & 5.6 \\
\hline $23,5,130-132$ & 203.31 & .11 & +78.8 & 115.9 & +70.6 & 111.1 & $\mathrm{~N}$ & 4.3 \\
\hline $23,6,9-11$ & 203.60 & .14 & +80.9 & 109.6 & +73.5 & 109.4 & $\mathrm{~N}$ & 4.2 \\
\hline $23,6,39-41$ & 203.90 & .16 & +83.1 & 68.1 & +67.2 & 111.5 & $\mathrm{~N}$ & 12.4 \\
\hline $23,6,69-71$ & 204.20 & .13 & +79.8 & 236.6 & +83.7 & 131.0 & $\mathrm{~N}$ & 4.9 \\
\hline $23,6,101-103$ & 204.52 & .21 & +87.5 & 160.8 & +79.5 & 110.9 & $\mathrm{~N}$ & 10.7 \\
\hline $23,6,132-134$ & 204.83 & .18 & +67.8 & 103.5 & -68.9 & 150.4 & $\mathrm{R}$ & 6.4 \\
\hline $23,7,9-11$ & 205.10 & .32 & +77.1 & 102.1 & -69.7 & 254.1 & $\mathrm{R}$ & 7.3 \\
\hline $23,7,39-41$ & 205.40 & .57 & +78.2 & 65.1 & -88.6 & 228.7 & R & 11.4 \\
\hline $24,1,70-72$ & 206.31 & .04 & +81 & & +7 & & & 5.4 \\
\hline $24,1,99-101$ & 206.60 & .08 & +82.2 & & -44 & .4 & $\mathrm{R}$ & 3.3 \\
\hline $24,1,130-132$ & 206.91 & .07 & +46.5 & 101.9 & -78.3 & 262.6 & $\mathrm{R}$ & \\
\hline $24,2,10-12$ & 207.21 & .04 & +82.3 & 12.5 & -71.1 & 275.3 & $\mathrm{R}$ & 3.9 \\
\hline $24,2,40-42$ & 207.51 & 05 & +71.5 & 137 & -60.4 & 310.4 & $\mathrm{R}$ & 3.3 \\
\hline $24,2,70-72$ & 207.81 & .04 & +75.7 & 28.6 & -78.7 & 331.8 & $\mathrm{R}$ & 3.4 \\
\hline $24,2,99-101$ & 208.10 & .07 & -44.1 & 108.7 & -65.3 & 286.7 & $\mathrm{R}$ & 5.3 \\
\hline $24,2,130-132$ & 208.41 & .16 & -50.8 & 269.1 & -64.1 & 279.2 & $\mathrm{R}$ & 4.1 \\
\hline $24,3,10-12$ & 208.71 & .13 & -29.7 & 163.3 & -74.4 & 261.3 & $\mathrm{R}$ & 11.8 \\
\hline $24,3,40-42$ & 209.01 & .04 & +59.6 & 74.3 & -73.3 & 245.9 & $\mathrm{R}$ & 10.0 \\
\hline $24,3,70-72$ & 209.31 & .01 & -71.3 & 201.8 & -58.3 & 219.6 & $\mathrm{R}$ & \\
\hline $24,3,99-101$ & 209.60 & .04 & +47.0 & 143.3 & +65.1 & 150.2 & $\mathrm{~N}$ & 2.1 \\
\hline $24,4,10-12$ & 210.21 & .10 & +29.2 & 106.9 & +60.1 & 50.6 & $\mathrm{~N}$ & 2.7 \\
\hline $24,4,40-42$ & 210.51 & .20 & +35.1 & 102.2 & +58.0 & 18.2 & $\mathrm{~N}$ & 3.4 \\
\hline $24,4,70-72$ & 210.81 & .02 & +71.7 & 181.9 & -40.7 & 261.7 & $\mathrm{R}$ & 2.1 \\
\hline $24,4,99-101$ & 211.10 & .07 & -25.7 & 82.0 & -84.9 & 305.0 & $\mathrm{R}$ & 4.2 \\
\hline $24,4,130-132$ & 211.41 & .03 & -64.1 & 91.4 & -70.1 & 292.2 & $\mathrm{R}$ & 3.1 \\
\hline $24,5,10-12$ & 211.71 & .11 & -58.6 & 277.9 & -61.3 & 283.8 & $\mathrm{R}$ & \\
\hline $24,5,40-42$ & 212.01 & .06 & +8.4 & 160.4 & -73.2 & 267.1 & $\mathrm{R}$ & 3.1 \\
\hline $24,5,70-72$ & 212.31 & .05 & +86.2 & 167.0 & -71.7 & 274.5 & $\mathrm{R}$ & 2.9 \\
\hline $24,5,99-101$ & 212.60 & .40 & -7.5 & 251.5 & -60.1 & 262.3 & $\mathrm{R}$ & 3.2 \\
\hline $24,5,130-132$ & 212.91 & .57 & -14.6 & 89.4 & -15.4 & 278.2 & $R$ & 3.1 \\
\hline $25,1,40-42$ & 213.51 & .14 & -31.7 & 208.2 & -29.9 & & R & 2.8 \\
\hline $25,1,70-72$ & 213.81 & .06 & -38.2 & 246.2 & -57.8 & 209.8 & $\mathrm{R}$ & 27.8 \\
\hline $25,1,100-102$ & 214.11 & .02 & +21.2 & 36.9 & +79.7 & 45.6 & $\mathrm{~N}$ & \\
\hline $25,1,130-132$ & 214.41 & .04 & +7.8 & 118.0 & +60.1 & 18.3 & $\mathrm{~N}$ & 6.3 \\
\hline $25,2,10-12$ & 214.71 & .01 & +48.0 & 33.5 & +61.2 & 349.0 & $\mathrm{~N}$ & \\
\hline $25,2,40-42$ & 215.01 & .01 & -12.2 & 295.9 & +62.7 & 3.7 & $\mathrm{~N}$ & \\
\hline $25,2,70-72$ & 215.31 & .01 & +56.7 & 258.9 & -63.9 & 223.4 & & \\
\hline $25,2,100-102$ & 215.61 & .02 & +23.1 & 333.5 & +66.1 & 326.5 & $\mathrm{~N}$ & \\
\hline $25,2,130-132$ & 215.91 & .03 & -54.1 & 347.7 & +56.4 & 353.2 & $\mathrm{~N}$ & \\
\hline $25,3,10-12$ & 216.21 & .03 & +13.9 & 313.7 & +60.4 & 337.2 & $\mathrm{~N}$ & \\
\hline $25,3,40-42$ & 216.51 & .05 & +65.1 & 317.9 & +77.3 & 347.2 & $\mathrm{~N}$ & 8.9 \\
\hline
\end{tabular}


Table A1 (continued).

\begin{tabular}{|c|c|c|c|c|c|c|c|c|}
\hline $\begin{array}{l}\text { Core, Section, } \\
\text { Interval }(\mathrm{cm})\end{array}$ & $\begin{array}{l}\text { Depth } \\
\text { (mbsf) }\end{array}$ & $\begin{array}{c}\mathrm{J} \\
\mathrm{NRM} \\
(\mathrm{mA} / \mathrm{m})\end{array}$ & $\begin{array}{l}\text { Incl. } \\
\text { NRM } \\
\text { (deg) }\end{array}$ & $\begin{array}{l}\text { Decl. } \\
\text { NRM } \\
\text { (deg) }\end{array}$ & $\begin{array}{l}\text { Incl } \\
\text { stable } \\
\text { (deg) }\end{array}$ & $\begin{array}{l}\text { Decl. } \\
\text { stable } \\
\text { (deg) }\end{array}$ & Pol. & $\begin{array}{l}\mathrm{MDF} \\
(\mathrm{mT})\end{array}$ \\
\hline $25,3,70-72$ & 216.81 & .73 & +67.8 & 5.4 & +63.7 & 355.3 & $\mathrm{~N}$ & 13.8 \\
\hline $25,3,100-102$ & 217.11 & .02 & +73.9 & 276.7 & +71.8 & .8 & $\mathrm{~N}$ & 9.1 \\
\hline $25,3,130-132$ & 217.41 & .01 & +27.3 & 49.9 & +66.9 & 356.1 & $\mathrm{~N}$ & 12.1 \\
\hline $25,4,10-12$ & 217.71 & .10 & +65.2 & 48.2 & +74.7 & 24.9 & $\mathrm{~N}$ & 20.4 \\
\hline $25,4,40-42$ & 218.01 & .01 & +29.1 & 345.6 & +68.8 & 339.9 & $\mathrm{~N}$ & \\
\hline $25,4,70-72$ & 218.31 & .02 & +56.0 & 64.7 & +65.6 & 21.9 & $\mathrm{~N}$ & 9.1 \\
\hline $25,4,100-102$ & 218.61 & .02 & +76.3 & 12.9 & +60.4 & 7.9 & $\mathrm{~N}$ & \\
\hline $25,4,130-132$ & 218.91 & .06 & +88.6 & 61.2 & +73.0 & 13.6 & $\mathrm{~N}$ & 4.0 \\
\hline $25,5,10-12$ & 219.21 & .08 & +72.3 & 114.5 & +74.0 & 6.1 & $\mathrm{~N}$ & 8.7 \\
\hline $25,5,40-42$ & 219.51 & .05 & +66.3 & 100.3 & +55.4 & 303.1 & $\mathrm{~N}$ & 5.5 \\
\hline $25,5,70-72$ & 219.81 & .01 & +37.4 & 156.9 & -59.0 & 162.1 & $\mathrm{R}$ & \\
\hline $25,5,100-102$ & 220.11 & .06 & -41.1 & 244.5 & -51.3 & 226.2 & $\mathrm{R}$ & 2.1 \\
\hline $25,5,130-132$ & 220.41 & .14 & +53.0 & 177.0 & -67.5 & 230.0 & $\mathrm{R}$ & \\
\hline $25,6,10-12$ & 220.71 & .03 & +3.1 & 186.4 & -50.7 & 216.0 & $\mathrm{R}$ & \\
\hline $25,6,40-42$ & 221.01 & .04 & +76.2 & 300.0 & +74.9 & 31.1 & $\mathrm{~N}$ & 12.2 \\
\hline
\end{tabular}

$\mathrm{J}_{\mathrm{NRM}}$, Decl.NRM, Incl.NRM - intensity, inclination, declination of the natural remanent magnetization; Incl.stable, Decl.stable inclination, declination of the stable remanence after AF demagnetization; Pol., N, R - polarity, normal, reversed; MDF - median destructive field. 
Table A2. Paleomagnetic properties of samples from Hole $642 \mathrm{C}$.

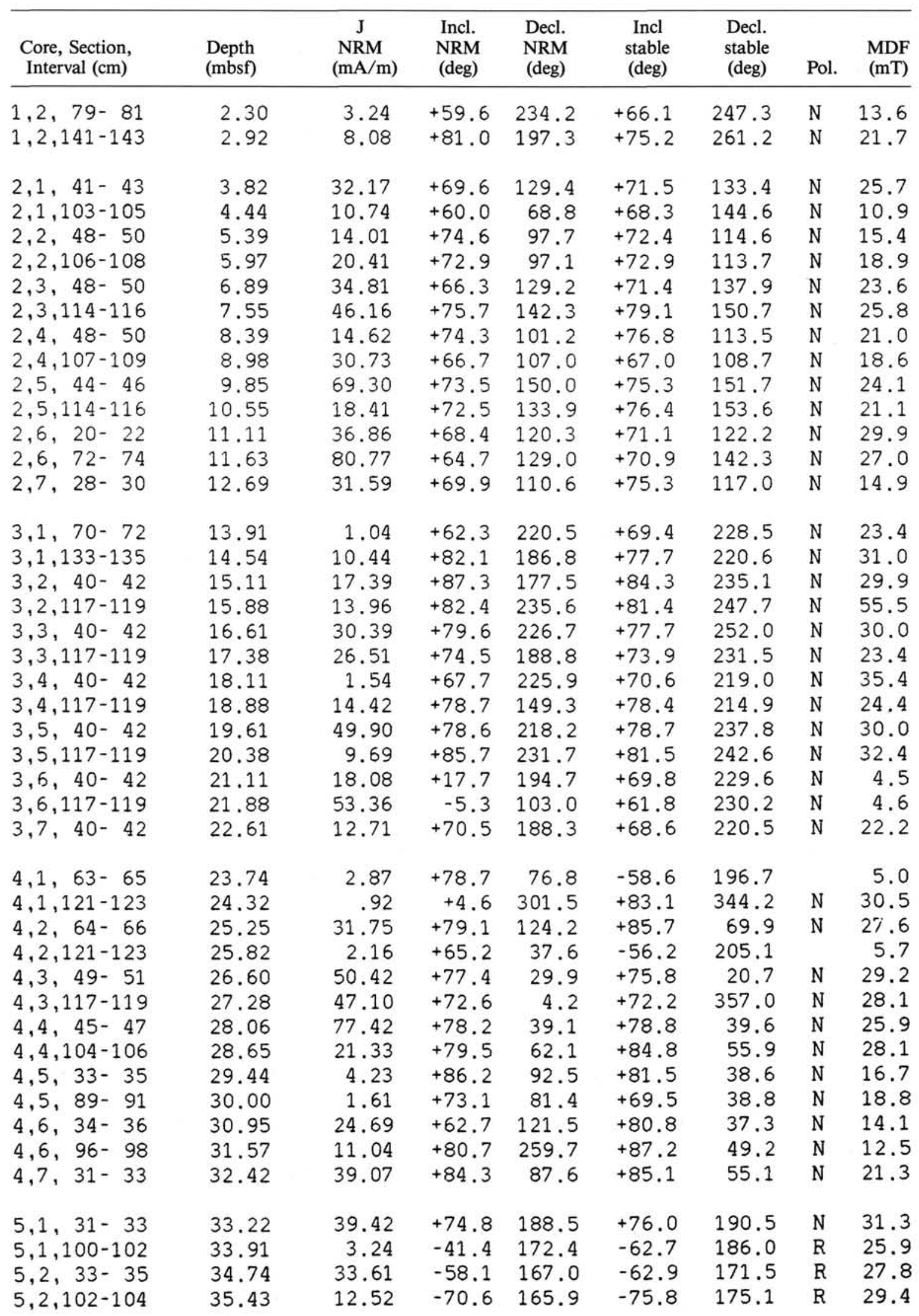


Table A2 (continued).

\begin{tabular}{|c|c|c|c|c|c|c|c|c|}
\hline $\begin{array}{l}\text { Core, Section, } \\
\text { Interval }(\mathrm{cm})\end{array}$ & $\begin{array}{l}\text { Depth } \\
\text { (mbsf) }\end{array}$ & $\begin{array}{c}\mathrm{J} \\
\mathrm{NRM} \\
(\mathrm{mA} / \mathrm{m})\end{array}$ & $\begin{array}{l}\text { Incl. } \\
\text { NRM } \\
\text { (deg) }\end{array}$ & $\begin{array}{l}\text { Decl. } \\
\text { NRM } \\
\text { (deg) }\end{array}$ & $\begin{array}{l}\text { Incl } \\
\text { stable } \\
(\mathrm{deg})\end{array}$ & $\begin{array}{l}\text { Decl. } \\
\text { stable } \\
\text { (deg) }\end{array}$ & Pol. & $\begin{array}{l}\mathrm{MDF} \\
(\mathrm{mT})\end{array}$ \\
\hline $5,3,31-33$ & 36.22 & 1.26 & -25.3 & 80.6 & -65.1 & 124.2 & $\mathrm{R}$ & 5.4 \\
\hline $5,3,100-102$ & 36.91 & 59.55 & -67.0 & 140.6 & -71.7 & 144.9 & $\mathrm{R}$ & 27.9 \\
\hline $5,4,32-34$ & 37.73 & 28.09 & -68.8 & 155.0 & -71.5 & 161.1 & R & 24.1 \\
\hline $5,4,100-102$ & 38.41 & .43 & -44.8 & 151.5 & -65.4 & 140.8 & $\mathrm{R}$ & 4.8 \\
\hline $5,5,32-34$ & 39.23 & 1.84 & -55.3 & 89.4 & -70.0 & 159.9 & $\mathrm{R}$ & 20.0 \\
\hline $5,5,100-102$ & 39.91 & 12.19 & +55.4 & 11.1 & +65.0 & 351.2 & $\mathrm{~N}$ & 38.1 \\
\hline $5,6,32-34$ & 40.73 & 10.29 & +68.5 & 35.6 & +77.8 & 22.9 & $\mathrm{~N}$ & 26.0 \\
\hline $6,1,42-44$ & 41.83 & 34.50 & +83.0 & 134.1 & +83.5 & 169.2 & N & 26.4 \\
\hline $6,1,118-120$ & 42.59 & .89 & +67.5 & 104.5 & 3.0 & 200.9 & $\mathrm{~N}$ & 13.9 \\
\hline $6,2,44-46$ & 43.35 & 21.86 & -71.5 & 59.1 & -77.3 & 21.3 & $\mathrm{R}$ & 37.4 \\
\hline $6,2,119-121$ & 44.10 & 6.93 & -55.9 & 19.7 & -66.4 & 1.6 & $\mathrm{R}$ & 37.8 \\
\hline $6,3,42-44$ & 44.83 & 3.74 & +80.5 & 109.4 & -63.3 & 43.3 & $\mathrm{R}$ & 3.3 \\
\hline $6,3,118-120$ & 45.59 & 26.05 & -75.9 & 341.3 & .9 & 342.6 & R & 27.8 \\
\hline $6,4,42-44$ & 46.33 & 14.24 & -66.8 & 350.1 & .8 & 354.6 & $\pi$ & 35.5 \\
\hline $6,4,118-120$ & 47.09 & 17.39 & -64.7 & 19.4 & .0 & 358.9 & $\mathrm{R}$ & 28.7 \\
\hline $6,5,43-45$ & 47.84 & 19.99 & -61.9 & 26.8 & -66.9 & 7.6 & $\mathrm{R}$ & 35.2 \\
\hline $6,5,118-120$ & 48.59 & 33.27 & -71.3 & 8.7 & -70.6 & 356.1 & R & 31.4 \\
\hline $6,6,41-43$ & 49.32 & 5.41 & -9.1 & 328.7 & -68.9 & 341.8 & $\mathrm{R}$ & 59.6 \\
\hline $6,6,118-120$ & 50.09 & 31.02 & -70.1 & 353.5 & -70.7 & 351.9 & $\mathrm{R}$ & 29.7 \\
\hline $7,1,40-42$ & 51.31 & 8.04 & -27.0 & 140.6 & -.3 & 136.6 & & 24.4 \\
\hline $7,1,109-111$ & 52.00 & .42 & +38.4 & 101.9 & +65.4 & 132.7 & $\mathrm{~N}$ & 9.3 \\
\hline $7,2,40-42$ & 52.81 & 21.18 & +87.9 & 41.6 & +85.4 & 209.0 & $\mathrm{~N}$ & 22.9 \\
\hline $7,2,112-114$ & 53.53 & 12.61 & +83.1 & 190.4 & +83.8 & 186.6 & $\mathrm{~N}$ & 19.3 \\
\hline $7,3,40-42$ & 54.31 & 2.06 & -53.2 & 35.2 & -66.6 & 21.9 & $\mathrm{R}$ & 42.2 \\
\hline $7,3,112-114$ & 55.03 & 2.46 & -12.8 & 98.6 & -70.4 & 35.9 & R & 7.9 \\
\hline $7,4,40-42$ & 55.81 & 19.92 & -73.8 & 65.3 & -68.5 & 50.4 & $\mathrm{R}$ & 22.1 \\
\hline $7,4,112-114$ & 56.53 & 23.67 & -82.5 & 75.5 & -83.5 & 49.0 & $\mathrm{R}$ & 28.3 \\
\hline $8,1,40-42$ & 57.51 & 82.84 & +68.6 & 51.4 & +67.1 & 52.8 & $\mathrm{~N}$ & 30.1 \\
\hline $8,1,110-112$ & 58.21 & 5.14 & +54.7 & 128.8 & +67.6 & 31.6 & $\mathrm{~N}$ & 28.6 \\
\hline $8,2,40-42$ & 59.01 & 4.40 & -70.9 & 188.7 & -72.4 & 190.9 & $\mathrm{R}$ & 60.6 \\
\hline $8,2,110-112$ & 59.71 & 9.14 & -66.8 & 170.2 & -72.9 & 174.1 & $\mathrm{R}$ & 33.1 \\
\hline $8,3,40-42$ & 60.51 & 23.12 & -84.2 & 163.6 & -80.5 & 167.5 & $\mathrm{R}$ & 20.3 \\
\hline $9,1,40-42$ & 61.11 & 6.00 & -65.5 & 132.7 & -60.3 & 165.6 & $\mathrm{R}$ & 51.1 \\
\hline $9,1,110-112$ & 61.81 & 9.97 & -85.9 & 144.7 & -84.1 & 150.3 & $\mathrm{R}$ & 23.6 \\
\hline $0,1,40-42$ & 63.91 & 43.02 & +85.3 & 104.7 & +87.4 & 179.7 & $\mathrm{~N}$ & 31.1 \\
\hline $10,1,110-112$ & 64.61 & 15.72 & +77.8 & 167.0 & +76.5 & 181.4 & $\mathrm{~N}$ & 27.4 \\
\hline $10,6,42-44$ & 71.43 & 4.39 & -54.8 & 228.9 & -70.4 & 271.2 & $\mathrm{R}$ & 67.1 \\
\hline $10,6,110-112$ & 72.11 & 12.25 & -68.5 & 259.0 & -73.6 & 253.4 & $\mathrm{R}$ & 51.5 \\
\hline $10,7,15-17$ & 72.66 & 17.53 & -82.1 & 236.1 & -79.5 & 261.2 & $\mathrm{R}$ & 48.5 \\
\hline $11,1,40-42$ & 73.41 & 33.45 & -74.6 & 75.0 & -75.4 & 71.1 & $\mathrm{R}$ & 31.6 \\
\hline $11,1,110-112$ & 74.11 & 26.11 & -74.7 & 92.5 & -81.7 & 77.9 & $\mathrm{R}$ & 35.3 \\
\hline $11,2,40-42$ & 74.91 & 25.16 & -58.0 & 68.4 & -68.8 & 65.0 & $\mathrm{R}$ & 39.0 \\
\hline $11,2,110-112$ & 75.61 & 21.33 & -75.3 & 93.3 & -79.5 & 75.3 & $\mathrm{R}$ & 38.5 \\
\hline $11,3,40-42$ & 76.41 & 21.44 & -79.3 & 32.7 & -77.3 & 52.2 & $\mathrm{R}$ & 35.4 \\
\hline
\end{tabular}


Table A2 (continued).

\begin{tabular}{|c|c|c|c|c|c|c|c|c|}
\hline $\begin{array}{l}\text { Core, Section, } \\
\text { Interval }(\mathrm{cm})\end{array}$ & $\begin{array}{l}\text { Depth } \\
\text { (mbsf) }\end{array}$ & $\underset{(\mathrm{mA} / \mathrm{m})}{\mathrm{J}}$ & $\begin{array}{l}\text { Incl. } \\
\text { NRM } \\
\text { (deg) }\end{array}$ & $\begin{array}{l}\text { Decl. } \\
\text { NRM } \\
\text { (deg) }\end{array}$ & $\begin{array}{c}\text { Incl } \\
\text { stable } \\
\text { (deg) }\end{array}$ & $\begin{array}{l}\text { Decl. } \\
\text { stable } \\
\text { (deg) }\end{array}$ & Pol. & $\begin{array}{l}\mathrm{MDF} \\
(\mathrm{mT})\end{array}$ \\
\hline $11,3,110-112$ & 77.11 & 5.27 & +43.6 & 100.0 & -67.5 & 65.5 & $\mathrm{R}$ & 5.0 \\
\hline $11,4,40-42$ & 77.91 & 3.85 & -78.9 & 104.9 & -84.0 & 45.7 & $\mathrm{R}$ & 22.6 \\
\hline $11,4,110-112$ & 78.61 & 7.55 & -79.8 & 78.9 & -79.6 & 41.5 & $\mathrm{R}$ & 52.5 \\
\hline $11,5,40-42$ & 79.41 & .18 & -22.3 & 164.5 & -79.7 & 39.3 & $\mathrm{R}$ & \\
\hline $11,5,110-112$ & 80.11 & .09 & +75.6 & 210.8 & +66.9 & 182.6 & $\mathrm{~N}$ & 30.9 \\
\hline $11,6,40-42$ & 80.91 & 1.23 & +67.3 & 151.8 & +71.2 & 166.6 & $\mathrm{~N}$ & 47.7 \\
\hline $11,6,110-112$ & 81.61 & .28 & -17.4 & 28.0 & +63.8 & 171.4 & $\mathrm{~N}$ & 4.6 \\
\hline $11,7,40-42$ & 82.41 & 1.39 & +1.7 & 180.6 & +67.1 & 156.9 & $\mathrm{~N}$ & 3.3 \\
\hline $12,1,40-42$ & 82.91 & 6.64 & +57.9 & 168.8 & +82.6 & 23.4 & $\mathrm{~N}$ & 52.8 \\
\hline $12,1,110-112$ & 83.61 & 1.49 & +19.6 & 286.0 & +71.7 & 8.9 & $\mathrm{~N}$ & 3.1 \\
\hline $12,2,40-42$ & 84.41 & 2.08 & +73.1 & 177.9 & +73.8 & 4.9 & $\mathrm{~N}$ & 3.4 \\
\hline $12,2,110-112$ & 85.11 & .03 & +41.5 & 174.5 & -79.9 & 197.3 & $\mathrm{R}$ & \\
\hline $12,3,40-42$ & 85.91 & .05 & -4.2 & 97.8 & -82.2 & 182.2 & $\mathrm{R}$ & \\
\hline $12,3,110-112$ & 86.61 & .17 & +81.2 & 221.9 & -73.1 & 187.1 & $\mathrm{R}$ & 3.3 \\
\hline $12,4,40-42$ & 87.41 & .48 & -65.9 & 148.0 & -68.1 & 169.4 & $\mathrm{R}$ & \\
\hline $12,4,110-112$ & 88.11 & .12 & -2.1 & 165.3 & -72.1 & 168.8 & $\mathrm{R}$ & \\
\hline $12,5,40-42$ & 88.91 & .09 & -26.6 & 149.4 & -75.2 & 174.0 & $\mathrm{R}$ & \\
\hline $12,5,110-112$ & 89.61 & .32 & -26.8 & 147.6 & -70.2 & 186.9 & $\mathrm{R}$ & \\
\hline $12,6,40-42$ & 90.41 & .68 & -25.0 & 35.8 & -64.4 & 147.7 & $\mathrm{R}$ & \\
\hline $12,6,110-112$ & 91.11 & 5.74 & -66.7 & 174.5 & -70.4 & 169.8 & $\mathrm{R}$ & \\
\hline $13,1,40-42$ & 92.41 & 1.63 & +22.8 & .8 & .6 & 216.4 & $\mathrm{R}$ & 28.9 \\
\hline $13,1,110-112$ & 93.11 & .93 & +71.9 & 155.8 & -71.6 & 217.2 & $\mathrm{R}$ & 14.7 \\
\hline $13,2,40-42$ & 93.91 & .10 & +64.5 & 78.1 & -70.8 & 164.4 & $\mathrm{R}$ & 23.5 \\
\hline $13,2,110-112$ & 94.61 & .16 & +52.7 & 153.0 & +67.9 & 121.5 & $\mathrm{~N}$ & 43.3 \\
\hline $13,3,40-42$ & 95.41 & .19 & +47.7 & 13.5 & +70.0 & 24.7 & $\mathrm{~N}$ & 33.4 \\
\hline $14,1,40-42$ & 101.91 & .34 & +88.2 & 88.1 & +84.9 & 16.4 & $\mathrm{~N}$ & 28.4 \\
\hline $14,1,110-112$ & 102.61 & .49 & +86.7 & 60.0 & +82.2 & 8.1 & $\mathrm{~N}$ & 8.9 \\
\hline $14,2,40-42$ & 103.41 & .66 & +76.5 & 16.6 & +77.8 & 357.1 & $\mathrm{~N}$ & 21.9 \\
\hline $14,2,110-112$ & 104.11 & .28 & +79.9 & 353.1 & +78.7 & 311.0 & $\mathrm{~N}$ & 4.7 \\
\hline $14,3,40-42$ & 104.91 & .25 & -18.7 & 33.9 & -68.2 & 124.3 & $\mathrm{R}$ & \\
\hline $14,3,110-112$ & 105.61 & .28 & +26.6 & 137.4 & -61.8 & 171.6 & $\mathrm{R}$ & 47.9 \\
\hline $14,4,40-42$ & 106.41 & .37 & -86.3 & 167.7 & -70.6 & 155.1 & $\mathrm{R}$ & 49.2 \\
\hline $14,4,110-112$ & 107.11 & 3.28 & +77.5 & 41.7 & +82.9 & 346.8 & $\mathrm{~N}$ & 27.4 \\
\hline $14,5,40-42$ & 107.91 & .43 & +69.4 & 44.7 & +72.4 & 329.3 & $\mathrm{~N}$ & 19.0 \\
\hline $14,5,110-112$ & 108.61 & .21 & +87.8 & 144.2 & +76.6 & 336.4 & $\mathrm{~N}$ & 18.9 \\
\hline $14,6,40-42$ & 109.41 & .25 & +80.1 & 93.5 & +80.7 & 9.1 & $\mathrm{~N}$ & 22.3 \\
\hline $14,6,110-112$ & 110.11 & .77 & +80.7 & 96.0 & +87.9 & 32.2 & $\mathrm{~N}$ & 33.4 \\
\hline $14,7,40-42$ & 110.91 & .25 & +77.6 & 33.1 & +75.5 & 10.4 & $\mathrm{~N}$ & 9.7 \\
\hline, $110-112$ & 112 & .07 & +81.9 & 27 & +81.2 & .4 & $\mathrm{~N}$ & \\
\hline $15,2,39-41$ & 112.90 & .05 & +35.2 & 272.5 & +71.8 & 286.9 & $\mathrm{~N}$ & \\
\hline $15,2,100-102$ & 113.51 & .25 & +80.5 & 359.1 & +73.6 & 291.0 & $\mathrm{~N}$ & \\
\hline $15,3,39-41$ & 114.40 & .34 & +72.5 & 343.2 & +76.5 & 336.6 & $\mathrm{~N}$ & 42.5 \\
\hline $15,3,110-112$ & 115.11 & .19 & +.6 & 349.1 & -80.1 & 77.8 & $\mathrm{R}$ & 20.9 \\
\hline $15,4,36-38$ & 115.87 & .17 & -76.0 & 104.5 & -72.3 & 101.8 & $\mathrm{R}$ & 32.7 \\
\hline $15,4,106-108$ & 116.57 & .05 & -17.3 & 39.0 & -68.4 & 47.4 & $\mathrm{R}$ & 28.9 \\
\hline $15,5,36-38$ & 117.37 & .11 & -14.8 & 40.7 & -72.5 & 42.3 & $\mathrm{R}$ & 4.0 \\
\hline
\end{tabular}


Table A2 (continued).

\begin{tabular}{|c|c|c|c|c|c|c|c|c|}
\hline $\begin{array}{l}\text { Core, Section, } \\
\text { Interval (cm) }\end{array}$ & $\begin{array}{l}\text { Depth } \\
\text { (mbsf) }\end{array}$ & $\begin{array}{c}\mathrm{J} \\
\mathrm{NRM} \\
(\mathrm{mA} / \mathrm{m})\end{array}$ & $\begin{array}{l}\text { Incl. } \\
\text { NRM } \\
\text { (deg) }\end{array}$ & $\begin{array}{l}\text { Decl. } \\
\text { NRM } \\
\text { (deg) }\end{array}$ & $\begin{array}{c}\text { Incl } \\
\text { stable } \\
\text { (deg) }\end{array}$ & $\begin{array}{l}\text { Decl. } \\
\text { stable } \\
\text { (deg) }\end{array}$ & Pol. & $\begin{array}{l}\text { MDF } \\
(\mathrm{mT}) \\
\end{array}$ \\
\hline $15,5,108-110$ & 118.09 & .22 & -40.2 & 19.4 & -72.9 & 30.0 & $\mathrm{R}$ & 11.1 \\
\hline $15,6,35-37$ & 118.86 & .88 & +50.2 & 201.9 & +65.9 & 156.1 & $\mathrm{~N}$ & 5.5 \\
\hline $15,6,110-112$ & 119.61 & 1.00 & -15.4 & 29.8 & +62.9 & 178.1 & $\mathrm{~N}$ & 3.1 \\
\hline $15,7,40-42$ & 120.41 & .75 & -22.3 & 25.1 & -77.2 & 29.1 & $\mathrm{R}$ & 3.3 \\
\hline $16,1,40-42$ & 120.91 & .99 & -41.0 & 359.2 & -73.1 & 194.4 & $\mathrm{R}$ & 3.1 \\
\hline $16,1,110-112$ & 121.61 & .42 & +77.8 & 342.7 & +75.3 & 19.1 & $\mathrm{~N}$ & 5.2 \\
\hline $16,2,40-42$ & 122. & .32 & +58.6 & 346.1 & +75.6 & 17.3 & N & 3.1 \\
\hline $16,2,110-112$ & 123.11 & .16 & +58.0 & 41.3 & +69.9 & 34.9 & $\mathrm{~N}$ & 5.1 \\
\hline $16,3,40-42$ & 123.91 & .11 & +86.1 & 135.6 & +70.7 & 19.8 & $\mathrm{~N}$ & 33.3 \\
\hline $16,3,110-112$ & 124.61 & .20 & +69.2 & 40.9 & +64 & 29.2 & $\mathrm{~N}$ & 3.0 \\
\hline $16,4,40-42$ & 125.41 & .40 & +73.8 & 14.0 & 4 & 10.3 & $\mathrm{~N}$ & 7.9 \\
\hline $16,4,110-112$ & 126.11 & .23 & +65.2 & 64.8 & 7 & 1 & N & 6.6 \\
\hline $16,5,40-42$ & 126.91 & .57 & +42.8 & 334.6 & 4 & 38.3 & N & 4.6 \\
\hline $16,5,110-112$ & 127.61 & .90 & +77.0 & .4 & + & 4 & $\mathrm{~N}$ & 27.7 \\
\hline $16,6,40-42$ & 128.41 & .36 & +78.7 & 17.9 & 3 & 6 & N & 11.9 \\
\hline $16,6,110-112$ & 129.11 & 2.74 & +81.1 & 318.0 & +81.5 & 48.3 & $\mathrm{~N}$ & 55.7 \\
\hline $17,1,62-64$ & $13 c$ & .19 & +68.2 & .9 & & 4 & N & 11.9 \\
\hline $17,1,122-124$ & 131.23 & .02 & -17.0 & 112.9 & + & 71.2 & $\mathrm{~N}$ & \\
\hline $17,2,27-29$ & 131.78 & .11 & +27.2 & 39.5 & 9 & 128.4 & $\mathrm{~N}$ & 2.3 \\
\hline $17,2,125-127$ & 132.76 & .49 & +69.6 & 360.0 & -6 & 6 & $\mathrm{R}$ & 17.8 \\
\hline $17,3,36-38$ & 133.37 & 3.87 & -8.4 & 195.6 & 0 & 267.0 & $\mathrm{R}$ & 3.8 \\
\hline $17,3,90-92$ & 133.91 & .12 & +16.8 & 133.0 & +7 & 79.0 & $\mathrm{~N}$ & 3.2 \\
\hline $17,5,33-35$ & 136.34 & .14 & +60.0 & 319.4 & +6 & 76.8 & $\mathrm{~N}$ & 16.3 \\
\hline $17,5,118-120$ & 137.19 & .32 & +4.4 & 207.4 & .1 & 91.9 & $\mathrm{~N}$ & 3.9 \\
\hline $17,6,26-28$ & 137.77 & .09 & +64.9 & 37.9 & +75.4 & 89.8 & $N$ & 10.6 \\
\hline $17,6,115-117$ & 138.66 & .06 & +27.2 & 25.6 & +66.1 & 82.3 & $\mathrm{~N}$ & 16.0 \\
\hline $18,1,60-62$ & 14 & .06 & +57.2 & 23.1 & +7 & 55 & N & 12.8 \\
\hline $18,1,121-123$ & 140.72 & .12 & .1 & 23.5 & & 28.4 & $\mathrm{~N}$ & 6.5 \\
\hline $18,2,50-52$ & 141.51 & .05 & +79.5 & 320.9 & +80.0 & 47.2 & $\mathrm{~N}$ & 2.1 \\
\hline $18,2,100-102$ & 142.01 & .05 & +60.6 & 344.1 & +65.6 & 305.4 & $\mathrm{~N}$ & 4.2 \\
\hline $18,3,50-52$ & 143.01 & .07 & +28.7 & 24.2 & -40.6 & 182.5 & $\mathrm{R}$ & 11.5 \\
\hline $18,3,100-102$ & 143.51 & .92 & +40.8 & 160.3 & -68.0 & 154.6 & $\mathrm{R}$ & 19.0 \\
\hline $18,4,50-52$ & 144.51 & .59 & +76.6 & 23.8 & +75.9 & 34.7 & $\mathrm{~N}$ & 23.7 \\
\hline $18,4,100-102$ & 145.01 & .14 & +69.2 & 21.9 & +71.7 & 8.4 & $\mathrm{~N}$ & 13.2 \\
\hline $18,5,50-52$ & 146.01 & .08 & +65.5 & 51.1 & +71.2 & 49.0 & $\mathrm{~N}$ & 9.2 \\
\hline $18,5,100-102$ & 146.51 & .05 & +85.1 & 340.0 & +73.1 & 47.6 & $\mathrm{~N}$ & 5.8 \\
\hline $18,6,20-22$ & 147.21 & .06 & +77.9 & 315.5 & +71.7 & 39.1 & $\mathrm{~N}$ & 11.6 \\
\hline $18,6,71-73$ & 147.72 & .15 & +74.6 & 347.5 & +82.2 & 39.6 & $\mathrm{~N}$ & 7.8 \\
\hline 19,1 & 145 & 3.47 & +1 & 13 & & & $\mathrm{~N}$ & 24.8 \\
\hline, $100-102$ & 150.01 & .13 & -20.2 & 56.8 & -44.7 & 284.5 & & \\
\hline $19,2,50-52$ & 151.01 & .19 & +78.6 & 20.1 & +75.5 & 18.0 & $\mathrm{~N}$ & 12.3 \\
\hline $19,2,110-112$ & 151.61 & .06 & +81.1 & 167.8 & +68.5 & 4.2 & N & 4.7 \\
\hline $19,3,32-34$ & 152.33 & .03 & +74.8 & 84.5 & +69.2 & 11.3 & $\mathrm{~N}$ & 4.9 \\
\hline $19,3,92-94$ & 152.93 & .04 & +52.0 & 145.2 & +79.2 & 15.6 & $\mathrm{~N}$ & 2.1 \\
\hline $19,4,33-35$ & 153.84 & .05 & +51.9 & 23.5 & +74.2 & 9.2 & $\mathrm{~N}$ & 13.3 \\
\hline $19,4,92-94$ & 154.43 & .05 & +74.7 & 34.0 & +76.7 & 36.3 & $\mathrm{~N}$ & 11.6 \\
\hline
\end{tabular}


Table A2 (continued).

\begin{tabular}{|c|c|c|c|c|c|c|c|c|}
\hline $\begin{array}{l}\text { Core, Section, } \\
\text { Interval }(\mathrm{cm})\end{array}$ & $\begin{array}{l}\text { Depth } \\
\text { (mbsf) }\end{array}$ & $\begin{array}{c}\mathrm{J} \\
\mathrm{NRM} \\
(\mathrm{mA} / \mathrm{m})\end{array}$ & $\begin{array}{l}\text { Incl. } \\
\text { NRM } \\
\text { (deg) }\end{array}$ & $\begin{array}{l}\text { Decl. } \\
\text { NRM } \\
\text { (deg) }\end{array}$ & $\begin{array}{c}\text { Incl } \\
\text { stable } \\
\text { (deg) }\end{array}$ & $\begin{array}{c}\text { Decl. } \\
\text { stable } \\
\text { (deg) }\end{array}$ & Pol. & $\begin{array}{l}\mathrm{MDF} \\
(\mathrm{mT})\end{array}$ \\
\hline $19,5,33-35$ & 155.34 & .03 & +60.3 & 341.3 & +70.4 & 48.0 & $\mathrm{~N}$ & 14.6 \\
\hline $19,5,95-97$ & 155.96 & .04 & +47.8 & 2.6 & +66.0 & 23.4 & $\mathrm{~N}$ & 16.4 \\
\hline $19,6,33-35$ & 156.84 & .07 & +57.3 & 68.9 & +78.1 & 53.2 & $\mathrm{~N}$ & 17.2 \\
\hline $19,6,95-97$ & 157.46 & .26 & +71.8 & 354.1 & +78.4 & 15.9 & $\mathrm{~N}$ & 30.8 \\
\hline $19,7,31-33$ & 158.32 & .23 & +79.3 & 315.5 & +81.0 & 26.4 & $\mathrm{~N}$ & 4.5 \\
\hline $20,1,45-47$ & 158.96 & .19 & +23.4 & 73.8 & +68.9 & 65.9 & $\mathrm{~N}$ & 59.5 \\
\hline $20,1,117-119$ & 159.68 & .08 & +62.2 & 353.5 & +69.2 & 5.7 & $\mathrm{~N}$ & 42.7 \\
\hline $20,2,45-47$ & 160.46 & .17 & +62.6 & 6.5 & +69.2 & 11.5 & $\mathrm{~N}$ & 17.9 \\
\hline $20,2,117-119$ & 161.18 & .02 & -4.2 & 52.6 & -51.2 & 167.9 & $\mathrm{R}$ & \\
\hline $20,3,47-49$ & 161.98 & .06 & +33.9 & 19.4 & +35.9 & 22.8 & $\mathrm{~N}$ & 6.9 \\
\hline $20,3,101-103$ & 162.52 & .12 & +46.1 & 53.7 & +71.8 & 27.9 & $\mathrm{~N}$ & 8.3 \\
\hline $20,4,45-47$ & 163.46 & .30 & +46.5 & 35.6 & +73.0 & 12.7 & $\mathrm{~N}$ & 13.9 \\
\hline $20,4,101-103$ & 164.02 & .17 & +50.2 & 46.8 & +6 & 33.3 & $\mathrm{~N}$ & 14.9 \\
\hline $20,5,47-49$ & 164.98 & .10 & +58.3 & 78.2 & +73 & 40.8 & $\mathrm{~N}$ & 17.1 \\
\hline $20,5,101-103$ & 165.52 & .12 & +40.7 & 27.2 & +69.5 & 18.4 & $\mathrm{~N}$ & 20.5 \\
\hline $20,6,47-49$ & 166.48 & .10 & +67.2 & 39.3 & +75.3 & 36.8 & $\mathrm{~N}$ & 22.5 \\
\hline $20,6,101-103$ & 167.02 & .34 & +3.7 & 290.8 & +67.2 & 19.5 & $\mathrm{~N}$ & 33.5 \\
\hline $20,7,47-49$ & 167.98 & 3.82 & +64.3 & 54.5 & +71.2 & 47.2 & $\mathrm{~N}$ & 33.3 \\
\hline $21,1,42-44$ & 43 & .17 & & 8 & .9 & 3.3 & $\mathrm{~N}$ & 17.2 \\
\hline $21,1,111-113$ & 169.12 & 1.72 & +74.8 & 60.2 & .9 & 60.3 & $\mathrm{~N}$ & 30.2 \\
\hline $21,2,42-44$ & 169.93 & .16 & +63.8 & 38.5 & +71.6 & 59.9 & $\mathrm{~N}$ & 17.5 \\
\hline $21,2,111-113$ & 170.62 & .14 & +68.2 & 62.5 & +71.0 & 88.6 & $\mathrm{~N}$ & 10.9 \\
\hline $21,3,42-44$ & 171.43 & .29 & +75.0 & 76.6 & +77.4 & 59.6 & $N$ & 21.9 \\
\hline $21,3,111-113$ & 172.12 & .26 & +85.8 & 121.3 & +81.7 & 70.3 & $\mathrm{~N}$ & 15.9 \\
\hline $21,4,42-44$ & 172.93 & .08 & +40.6 & 54.6 & +63.3 & 57.2 & $\mathrm{~N}$ & 4.0 \\
\hline $21,4,112-114$ & 173.63 & .18 & +71.2 & 43.1 & +69.5 & 52.2 & $\mathrm{~N}$ & 18.5 \\
\hline $21,5,43-45$ & 174.44 & .50 & +65.4 & 106.6 & +71.7 & 85.1 & $\mathrm{~N}$ & 39.7 \\
\hline $21,5,113-115$ & 175.14 & .20 & +70.8 & 78.7 & +69.2 & 77.9 & $\mathrm{~N}$ & 22.4 \\
\hline $21,6,43-45$ & 175.94 & .06 & +42.8 & 70.0 & +64.6 & 59.7 & $\mathrm{~N}$ & 8.2 \\
\hline $21,6,113-115$ & 176.64 & .08 & +69.0 & 37.1 & +71.0 & 68.0 & $\mathrm{~N}$ & 8.6 \\
\hline $21,7,43-45$ & 177.44 & .04 & +46.6 & 328.8 & +74.7 & 47.3 & $\mathrm{~N}$ & 14.0 \\
\hline $22,1,50-52$ & & .06 & +44.6 & 34 & & 78 & $\mathrm{~N}$ & 9.3 \\
\hline $22,1,110-112$ & 17 & .1 & +31.6 & 56 & +68.0 & 86 & $\mathrm{~N}$ & 12.7 \\
\hline $22,2,40-42$ & 179.41 & .07 & +61.6 & 338.0 & -44.5 & 246.3 & $\mathrm{R}$ & 23.2 \\
\hline $22,2,110-112$ & 180.11 & .10 & +22.8 & 197.8 & -70.4 & 250.0 & $\mathrm{R}$ & 6.0 \\
\hline $22,3,40-42$ & 180.91 & .06 & +27.5 & 295.4 & -64.3 & 285.7 & $\mathrm{R}$ & 5.4 \\
\hline $22,3,110-112$ & 181.61 & .07 & +54.5 & 21.7 & -77.6 & 286.5 & $\mathrm{R}$ & 19.5 \\
\hline $22,4,40-42$ & 182.41 & .15 & +31.4 & 339.9 & -79.6 & 51.8 & $R$ & \\
\hline $22,4,110-112$ & 183.11 & .99 & +70.7 & 65.9 & +77.8 & 58.3 & $\mathrm{~N}$ & 46.4 \\
\hline $22,5,40-42$ & 183.91 & .09 & +63.9 & 28.5 & +67.9 & 53.2 & $\mathrm{~N}$ & 36.9 \\
\hline $23,1,92-94$ & 185.23 & .57 & +65.0 & 333.8 & +71.7 & 14.0 & N & 16.7 \\
\hline $23,1,133-135$ & 185.64 & .11 & +31.6 & 10.7 & +7.7 & 46.6 & & 4.9 \\
\hline $23,2,28-30$ & 186.09 & .56 & +24.3 & 232.0 & -48.4 & 200.9 & $\mathrm{R}$ & 32.0 \\
\hline $23,2,103-105$ & 186.84 & .16 & +28.4 & 71.9 & -69.7 & 219.7 & $\mathrm{R}$ & 3.2 \\
\hline $23,3,28-30$ & 187.59 & .19 & +72.1 & 42.5 & +69.7 & 49.0 & $\mathrm{~N}$ & 18.0 \\
\hline $23,3,103-105$ & 188.34 & .23 & +70.9 & .2 & +72.1 & 36.1 & $\mathrm{~N}$ & 18.6 \\
\hline
\end{tabular}


Table A2 (continued).

\begin{tabular}{|c|c|c|c|c|c|c|c|c|}
\hline $\begin{array}{c}\text { Core, Section, } \\
\text { Interval }(\mathrm{cm})\end{array}$ & $\begin{array}{l}\text { Depth } \\
\text { (mbsf) }\end{array}$ & $\begin{array}{c}\mathrm{J} \\
\mathrm{NRM} \\
(\mathrm{mA} / \mathrm{m})\end{array}$ & $\begin{array}{l}\text { Incl. } \\
\text { NRM } \\
\text { (deg) }\end{array}$ & $\begin{array}{l}\text { Decl. } \\
\text { NRM } \\
\text { (deg) }\end{array}$ & $\begin{array}{c}\text { Incl } \\
\text { stable } \\
\text { (deg) }\end{array}$ & $\begin{array}{c}\text { Decl. } \\
\text { stable } \\
\text { (deg) }\end{array}$ & Pol. & $\begin{array}{l}\text { MDF } \\
(\mathrm{mT}) \\
\end{array}$ \\
\hline $23,4,41-43$ & 189.22 & .51 & +31.0 & 125.7 & +74.5 & 105.0 & $\mathrm{~N}$ & 7.5 \\
\hline $23,4,103-105$ & 189.84 & .12 & +87.2 & 163.8 & +82.5 & 94.9 & $\mathrm{~N}$ & 8.3 \\
\hline $23,5,36-38$ & 190.67 & .11 & +72.1 & 328.7 & +85.2 & 63.4 & $\mathrm{~N}$ & 8.1 \\
\hline $23,5,103-105$ & 191.34 & .16 & +75.3 & 338.6 & +71.0 & 69.3 & $\mathrm{~N}$ & 14.1 \\
\hline $24,1,51-53$ & 192.92 & .02 & +4.4 & 52.3 & -57.4 & 135.3 & $\mathrm{R}$ & 18.9 \\
\hline $24,1,117-119$ & 193.58 & .03 & +58.7 & 36.2 & -65.2 & 201.6 & $\mathrm{R}$ & 3.2 \\
\hline $24,2,51-53$ & 194.42 & .03 & -52.5 & 125.9 & -62.7 & 174.7 & $\mathrm{R}$ & 5.7 \\
\hline $24,2,117-119$ & 195.08 & .04 & +48.4 & 24.1 & -65.8 & 225.8 & $\mathrm{R}$ & \\
\hline $24,3,39-41$ & 195.80 & .21 & +72.4 & 105.3 & -63.1 & 177.0 & $\mathrm{R}$ & 17.4 \\
\hline $24,3,117-119$ & 196.58 & .09 & +48.2 & 119.8 & -19.5 & 158.9 & $\mathrm{R}$ & 14.5 \\
\hline $24,4,37-39$ & 197.28 & 1.20 & +47.5 & 42.5 & +60.4 & 18.0 & $\mathrm{~N}$ & 28.3 \\
\hline $24,4,100-102$ & 197.91 & .22 & +67.4 & 61.4 & +70.0 & 43.2 & $\mathrm{~N}$ & 35.2 \\
\hline $24,5,39-41$ & 198.80 & .08 & +74.6 & 47.5 & +77.1 & 37.3 & in & 36.3 \\
\hline $24,5,104-106$ & 199.45 & .11 & +82.9 & 82.1 & +82.1 & 14.4 & $\mathrm{~N}$ & 14.1 \\
\hline
\end{tabular}

$\mathrm{J}_{\mathrm{NRM}}$, Decl. NRM $_{\text {, Incl.NRM }}$ - intensity, inclination, declination of the natural remanent magnetization; Incl.stable, Decl.stable inclination, declination of the stable remanence after AF demagnetization; Pol., N, R - polarity, normal, reversed; MDF - median destructive field. 
Table A3. Paleomagnetic properties of samples from Hole 642D.

\begin{tabular}{|c|c|c|c|c|c|c|c|c|}
\hline $\begin{array}{l}\text { Core, Section, } \\
\text { Interval }(\mathrm{cm})\end{array}$ & $\begin{array}{l}\text { Depth } \\
\text { (mbsf) }\end{array}$ & $\begin{array}{c}\mathrm{J} \\
\mathrm{NRM} \\
(\mathrm{mA} / \mathrm{m})\end{array}$ & $\begin{array}{l}\text { Incl. } \\
\text { NRM } \\
\text { (deg) }\end{array}$ & $\begin{array}{l}\text { Decl. } \\
\text { NRM } \\
\text { (deg) }\end{array}$ & $\begin{array}{c}\text { Incl } \\
\text { stable } \\
\text { (deg) }\end{array}$ & $\begin{array}{l}\text { Decl. } \\
\text { stable } \\
\text { (deg) }\end{array}$ & Pol. & $\begin{array}{l}\text { MDF } \\
(\mathrm{mT})\end{array}$ \\
\hline $2,1,40-42$ & 190.31 & .37 & -40.5 & 314.7 & -57.8 & 321.6 & $\mathrm{R}$ & 25.2 \\
\hline $2,1,110-112$ & 191.01 & .06 & +52.1 & 248.3 & -58.2 & 313.8 & $\mathrm{R}$ & 14.2 \\
\hline $2,2,40-42$ & 191.81 & .01 & +49.2 & 209.2 & -70.1 & 294.9 & $\mathrm{R}$ & \\
\hline $2,2,110-112$ & 192.51 & .04 & +46.6 & 224.4 & -78.5 & 341.9 & $\mathrm{R}$ & 8.6 \\
\hline $2,3,40-42$ & 193.31 & .02 & +56.5 & 231.9 & -65.8 & 326.1 & $\mathrm{R}$ & 3.6 \\
\hline $2,3,110-112$ & 194.01 & .05 & +75.7 & 217.4 & -70.4 & 289.6 & $\mathrm{R}$ & 3.3 \\
\hline $2,4,40-42$ & 194.81 & .04 & +79.1 & 266.5 & -68.8 & 351.8 & $\mathrm{R}$ & 14.7 \\
\hline $2,4,110-112$ & 195.51 & .04 & +85.2 & 103.7 & +79.5 & 75.1 & & 8.7 \\
\hline $2,5,40-42$ & 196.31 & .11 & +68.5 & 207.4 & -54.2 & 315.6 & $\mathrm{R}$ & 23.5 \\
\hline $2,5,110-112$ & 197.01 & .08 & +79.1 & 307.5 & +75.7 & 127.3 & $\mathrm{~N}$ & 23.5 \\
\hline $2,6,40-42$ & 197.81 & .07 & +72.8 & 144.3 & +78.5 & 133.6 & $\mathrm{~N}$ & 24.7 \\
\hline $2,6,110-112$ & 198.51 & .13 & +52.6 & 123.7 & +66.2 & 122.3 & $\mathrm{~N}$ & 18.5 \\
\hline $3,1,40-42$ & 200.01 & .07 & +53.8 & 95.8 & +65.7 & 111.5 & $\mathrm{~N}$ & 18.6 \\
\hline $3,1,110-112$ & 200.71 & .04 & +82.9 & 293.2 & +67.5 & 128.1 & $\mathrm{~N}$ & 14.1 \\
\hline $3,2,40-42$ & 201.51 & .04 & +73.6 & 153.8 & +75.0 & 139.0 & $\mathrm{~N}$ & 14.4 \\
\hline $3,2,110-112$ & 202.21 & .07 & +66.4 & 79.3 & +78.5 & 126.7 & $\mathrm{~N}$ & 5.8 \\
\hline $3,3,40-42$ & 203.01 & .08 & +52.8 & 140.0 & +69.1 & 139.7 & $\mathrm{~N}$ & 17.2 \\
\hline $3,3,110-112$ & 203.71 & .04 & +51.5 & 128.2 & +72.2 & 124.2 & $\mathrm{~N}$ & \\
\hline $3,4,40-42$ & 204.51 & .24 & +59.9 & 136.1 & +62.5 & 130.4 & $\mathrm{~N}$ & 16.8 \\
\hline $3,4,110-112$ & 205.21 & .55 & +80.2 & 224.6 & +86.9 & 130.2 & $\mathrm{~N}$ & 47.2 \\
\hline $3,5,40-42$ & 206.01 & .04 & +4.1 & 14.1 & -34.6 & 318.5 & $\mathrm{R}$ & 3.7 \\
\hline $3,5,110-112$ & 206.71 & .02 & -14.9 & 102.9 & -70.4 & 275.9 & $\mathrm{R}$ & \\
\hline $4,1,40-42$ & 209.61 & .03 & +37.3 & 340.7 & -67.9 & 51.1 & $\mathrm{R}$ & 24.0 \\
\hline $4,1,110-112$ & 210.31 & .02 & -57.3 & 51.1 & -69.2 & 56.5 & $\mathrm{R}$ & \\
\hline $4,2,40-42$ & 211.11 & .06 & -31.5 & 82.6 & -75.7 & 51.6 & $\mathrm{R}$ & 8.4 \\
\hline $4,2,110-112$ & 211.81 & .03 & +34.8 & 355.3 & -62.9 & 11.6 & $\mathrm{R}$ & 7.0 \\
\hline $4,3,40-42$ & 212.61 & .81 & +21.5 & 142.7 & +26.2 & 143.9 & $\mathrm{~N}$ & 17.0 \\
\hline $4,3,110-112$ & 213.31 & .02 & -17.8 & 140.4 & +72.7 & 244.6 & $\mathrm{~N}$ & \\
\hline $4,4,40-42$ & 214.11 & .10 & +33.5 & 268.6 & +62.9 & 231.8 & $\mathrm{~N}$ & 21.3 \\
\hline $4,4,110-112$ & 214.81 & .03 & +53.6 & 148.2 & +60.5 & 247.8 & $\mathrm{~N}$ & 3.5 \\
\hline $4,5,40-42$ & 215.61 & .04 & +59.6 & 195.6 & +75.8 & 250.5 & $\mathrm{~N}$ & \\
\hline $4,5,110-112$ & 216.31 & .06 & +59.3 & 220.7 & +85.8 & 243.1 & $\mathrm{~N}$ & 15.2 \\
\hline $4,6,40-42$ & 217.11 & .09 & +78.1 & 54.0 & +80.5 & 305.3 & $\mathrm{~N}$ & 2.8 \\
\hline $4,6,110-112$ & 217.81 & .06 & -60.0 & 336.9 & -70.4 & 332.4 & $\mathrm{R}$ & 4.9 \\
\hline $5,4,39-41$ & 223.80 & .05 & +38.3 & 97.3 & +61.9 & 76.9 & $\mathrm{~N}$ & 8.9 \\
\hline $5,4,109-111$ & 224.50 & .06 & +63.5 & 59.4 & +63.9 & 54.2 & $\mathrm{~N}$ & \\
\hline $5,6,39-41$ & 226.80 & .05 & +3.7 & 176.9 & +64.8 & 58.8 & $\mathrm{~N}$ & 3.5 \\
\hline $5,6,109-111$ & 227.50 & .01 & -7.4 & 74.0 & -50.8 & 198.4 & $R$ & \\
\hline $5,7,39-41$ & 228.30 & .02 & +73.6 & 80.1 & -60.7 & 228.2 & $\mathrm{R}$ & 6.0 \\
\hline $6,1,40-42$ & 228.91 & .10 & +77.1 & 159.6 & +74.2 & 119.0 & $\mathrm{~N}$ & 26.4 \\
\hline $6,1,130-132$ & 229.81 & .03 & +70.3 & 73.0 & +73.9 & 100.7 & $\mathrm{~N}$ & 6.7 \\
\hline $6,2,40-42$ & 230.41 & .04 & +82.4 & 76.0 & +61.8 & 105.1 & $\mathrm{~N}$ & 8.4 \\
\hline $6,2,110-112$ & 231.11 & .04 & +79.1 & 184.2 & +61.6 & 102.0 & $\mathrm{~N}$ & 4.1 \\
\hline $6,3,40-42$ & 231.91 & .14 & +79.1 & 61.1 & +83.1 & 119.5 & $\mathrm{~N}$ & 31.1 \\
\hline $6,3,110-112$ & 232.61 & .06 & +70.8 & 80.8 & -48.4 & 181.9 & $\mathrm{R}$ & 3.1 \\
\hline
\end{tabular}


Table A3 (continued).

\begin{tabular}{|c|c|c|c|c|c|c|c|c|}
\hline $\begin{array}{l}\text { Core, Section, } \\
\text { Interval }(\mathrm{cm})\end{array}$ & $\begin{array}{l}\text { Depth } \\
\text { (mbsf) }\end{array}$ & $\begin{array}{c}\mathrm{J} \\
\mathrm{NRM} \\
(\mathrm{mA} / \mathrm{m})\end{array}$ & $\begin{array}{l}\text { Incl. } \\
\text { NRM } \\
\text { (deg) }\end{array}$ & $\begin{array}{l}\text { Decl. } \\
\text { NRM } \\
\text { (deg) }\end{array}$ & $\begin{array}{l}\text { Incl } \\
\text { stable } \\
\text { (deg) }\end{array}$ & $\begin{array}{l}\text { Decl. } \\
\text { stable } \\
\text { (deg) }\end{array}$ & Pol. & $\begin{array}{l}\text { MDF } \\
(\mathrm{mT})\end{array}$ \\
\hline $6,4,40-42$ & 233.41 & .02 & -27.3 & 212.0 & -65.3 & 195.5 & $\mathrm{R}$ & 7.1 \\
\hline $6,4,110-112$ & 234.11 & .08 & -47.0 & 306.1 & -76.9 & 239.2 & $\mathrm{R}$ & \\
\hline $6,5,40-42$ & 234.91 & .09 & -10.4 & 81.8 & -66.8 & 252.8 & $\mathrm{R}$ & 3.8 \\
\hline $7,1,40-42$ & 238.61 & .07 & +76.2 & 154.8 & +77.8 & 195.7 & $\mathrm{~N}$ & \\
\hline $7,1,110-112$ & 239.31 & .10 & +82.4 & 100.1 & +73.0 & 222.3 & $\mathrm{~N}$ & 29.9 \\
\hline $7,2,40-42$ & 240.11 & .18 & +15.4 & 151.1 & +67.5 & 173.9 & $\mathrm{~N}$ & 3.8 \\
\hline $7,2,110-112$ & 240.81 & .53 & +84.0 & 261.3 & +83.9 & 216.2 & $\mathrm{~N}$ & 37.4 \\
\hline $7,3,40-42$ & 241.61 & .18 & +78.5 & 236.4 & +80.0 & 205.7 & $\mathrm{~N}$ & 18.6 \\
\hline $7,3,110-112$ & 242.31 & .07 & +43.2 & 192.8 & +65.6 & 206.5 & $\mathrm{~N}$ & 4.2 \\
\hline $7,4,40-42$ & 243.11 & .25 & +35.7 & 217.2 & +65.0 & 215.4 & $\mathrm{~N}$ & 6.4 \\
\hline $7,4,110-112$ & 243.81 & .44 & +61.1 & 57.0 & +71.6 & 176.2 & $\mathrm{~N}$ & 8.0 \\
\hline $7,5,40-42$ & 244.61 & .03 & +70.5 & 238.4 & +70.5 & 238.4 & $\mathrm{~N}$ & \\
\hline $7,5,110-112$ & 245.31 & .05 & +60.1 & 273.2 & +66.9 & 321.9 & $\mathrm{~N}$ & \\
\hline $7,6,40-42$ & 246.11 & .05 & -20.4 & 38.4 & +43.1 & 37.1 & $\mathrm{~N}$ & \\
\hline $7,6,110-112$ & 246.81 & .14 & -23.1 & 119.7 & -7 & 166.6 & & 3.4 \\
\hline $7,7,40-42$ & 247.61 & .33 & +15.2 & 120.8 & +85.7 & 273.7 & $\mathrm{~N}$ & 2.9 \\
\hline $8,1,40-42$ & 248.21 & .24 & +27.6 & 81.7 & 2 & 26.4 & $\mathrm{~N}$ & 2.9 \\
\hline $8,1,110-112$ & 248.91 & .50 & +34.7 & 65.3 & +72.6 & 14.2 & $\mathrm{~N}$ & 4.2 \\
\hline $8,2,40-42$ & 249.71 & .18 & +85.6 & 118.7 & +68.3 & 354.3 & $\mathrm{~N}$ & 37.6 \\
\hline $8,2,110-112$ & 250.41 & .07 & +65.6 & 281.2 & +69.9 & 22.7 & $\mathrm{~N}$ & \\
\hline $8,3,40-42$ & 251.21 & .19 & +6.4 & 359.2 & +71.2 & 45.9 & $\mathrm{~N}$ & \\
\hline $8,3,110-112$ & 251.91 & .22 & +71.3 & 350.8 & +77.5 & 356.7 & $\mathrm{~N}$ & 22.1 \\
\hline $8,4,40-42$ & 252.71 & .07 & +71.1 & 24.9 & +62.2 & 12.9 & $\mathrm{~N}$ & 7.6 \\
\hline $8,4,110-112$ & 253.41 & .04 & +59.5 & 48.4 & +56.8 & 37.0 & $\mathrm{~N}$ & 3.0 \\
\hline $8,5,40-42$ & 254.21 & .06 & +57.8 & 330.7 & +57.2 & 14.0 & $\mathrm{~N}$ & 10.6 \\
\hline $8,5,110-112$ & 254.91 & .05 & -54.7 & 69.2 & -62.0 & 48.7 & $\mathrm{R}$ & 4.2 \\
\hline $8,6,40-42$ & 255.71 & .05 & -79.3 & 237.3 & -74.6 & 245.2 & $\mathrm{R}$ & 13.0 \\
\hline $8,6,110-112$ & 256.41 & .12 & +61.9 & 291.8 & -64.5 & 261.7 & $\mathrm{R}$ & 9.2 \\
\hline $9,1,50-52$ & 258.01 & .04 & +78.6 & 1.7 & -6 & 56.9 & $\mathrm{R}$ & 4.3 \\
\hline $9,1,101-103$ & 258.52 & .14 & -57.4 & 16.2 & .5 & 34.4 & $\mathrm{R}$ & 20.4 \\
\hline $9,2,50-52$ & 259.51 & .02 & +69.3 & 314.6 & -75.1 & 42.2 & $\mathrm{R}$ & \\
\hline $9,2,101-103$ & 260.02 & .12 & +82.2 & 350.3 & +75.0 & 146.1 & $\mathrm{~N}$ & 20.3 \\
\hline $9,3,50-52$ & 261.01 & .12 & +69.0 & 109.9 & +67.9 & 197.2 & $\mathrm{~N}$ & 16.5 \\
\hline $9,3,101-103$ & 261.52 & .85 & +71.1 & 218.4 & +69.2 & 218.5 & $\mathrm{~N}$ & 15.4 \\
\hline $9,4,50-52$ & 262.51 & .02 & +77.2 & 184.4 & +66.2 & 216.6 & $\mathrm{~N}$ & \\
\hline $9,4,101-103$ & 263.02 & .05 & +78.2 & 159.9 & +72.8 & 221.1 & $\mathrm{~N}$ & 6.5 \\
\hline $9,5,50-52$ & 264.01 & .06 & +78.4 & 266.2 & -46.0 & 247.1 & $\mathrm{R}$ & 6.6 \\
\hline $9,5,101-103$ & 264.52 & .14 & +27.9 & 327.7 & -65.7 & 43.9 & $\mathrm{R}$ & 3.7 \\
\hline $9,6,50-52$ & 265.51 & .07 & -65.4 & 346.1 & -62.6 & 8.7 & $\mathrm{R}$ & \\
\hline $9,6,101-103$ & 266.02 & .23 & -56.9 & 79.2 & -67.6 & 85.7 & $\mathrm{R}$ & 3.9 \\
\hline 10,1 & 267 & .08 & +75.7 & 8.1 & -79.8 & 27 & $\mathrm{R}$ & 3.6 \\
\hline $10,1,119-121$ & 268.30 & .0 & +47.2 & 32.0 & -62.6 & 286.4 & $\mathrm{R}$ & 13.3 \\
\hline $10,2,45-47$ & 269.06 & .02 & +5.4 & 80.4 & -68.8 & 330.9 & $\mathrm{R}$ & \\
\hline $10,2,123-125$ & 269.84 & .04 & -8.0 & 106.3 & -61.9 & 299.4 & $\mathrm{R}$ & 4.6 \\
\hline $10,3,45-47$ & 270.56 & .07 & +15.3 & 111.7 & -66.6 & 334.2 & $\mathrm{R}$ & 34.2 \\
\hline $10,3,125-127$ & 271.36 & .03 & +33.9 & 317.8 & -65.8 & 344.7 & $\mathrm{R}$ & \\
\hline
\end{tabular}




\begin{tabular}{|c|c|c|c|c|c|c|c|c|}
\hline $\begin{array}{l}\text { Core, Section, } \\
\text { Interval }(\mathrm{cm})\end{array}$ & $\begin{array}{l}\text { Depth } \\
\text { (mbsf) }\end{array}$ & $\begin{array}{c}\mathrm{J} \\
\mathrm{NRM} \\
(\mathrm{mA} / \mathrm{m})\end{array}$ & $\begin{array}{l}\text { Incl. } \\
\text { NRM } \\
\text { (deg) }\end{array}$ & $\begin{array}{l}\text { Decl. } \\
\text { NRM } \\
\text { (deg) }\end{array}$ & $\begin{array}{c}\text { Incl } \\
\text { stable } \\
\text { (deg) }\end{array}$ & $\begin{array}{l}\text { Decl. } \\
\text { stable } \\
\text { (deg) }\end{array}$ & Pol. & $\begin{array}{l}\text { MDF } \\
(\mathrm{mT})\end{array}$ \\
\hline $10,4,42-44$ & 272.03 & .03 & +19.4 & 287.9 & -45.8 & 301.7 & $\mathrm{R}$ & \\
\hline $10,4,125-127$ & 272.86 & .09 & -69.4 & 308.8 & -66.8 & 306.5 & $R$ & 11.5 \\
\hline $10,5,42-44$ & 273.53 & .04 & -68.8 & 270.3 & -68.7 & 303.4 & $\mathrm{R}$ & \\
\hline $10,5,123-125$ & 274.34 & .08 & +82.4 & 291.1 & +67.6 & 104.3 & $\mathrm{~N}$ & 26.0 \\
\hline $10,6,48-50$ & 275.09 & .09 & +29.5 & 63.0 & +75.1 & 72.0 & $N$ & 15.7 \\
\hline $10,6,127-129$ & 275.88 & .05 & -5.1 & 54.3 & +68.4 & 42.9 & $\mathrm{~N}$ & 22.5 \\
\hline $11,1,33-35$ & 277.14 & .19 & +66.9 & 260.1 & +76.3 & 332.8 & $\mathrm{~N}$ & 11.1 \\
\hline $11,1,75-77$ & 277.56 & .40 & +81.8 & 4.4 & +74.2 & 339.4 & $\mathrm{~N}$ & 29.3 \\
\hline $11,1,108-110$ & 277.89 & 14.02 & +68.0 & 167.6 & -57.9 & 155.3 & $\mathrm{R}$ & 22.2 \\
\hline $11,2,33-35$ & 278.64 & 51.24 & +84.9 & 188.0 & -41.6 & 221.5 & $R$ & 8.0 \\
\hline $11,2,109-111$ & 279.40 & 1.76 & +40.0 & 138.7 & -79.8 & 213.6 & $\mathrm{R}$ & \\
\hline $12,1,39-41$ & .90 & 33.49 & .1 & .4 & 4 & 8 & $\mathrm{~N}$ & 9.5 \\
\hline $12,1,110-112$ & 287.61 & 18.80 & +75.0 & 86.6 & +7 & 48.6 & $\mathrm{~N}$ & 12.4 \\
\hline $12,2,39-41$ & 288.40 & 29.25 & +77.0 & 46.0 & +74.1 & 37.0 & $\mathrm{~N}$ & 13.0 \\
\hline $12,2,110-112$ & 289.11 & 9.95 & +76.5 & 52.6 & +74.6 & 29.4 & $\mathrm{~N}$ & 11.1 \\
\hline $12,3,39-41$ & 289.90 & 10.81 & +73.7 & 89.9 & +79.8 & 59.2 & $\mathrm{~N}$ & 7.3 \\
\hline $12,3,110-112$ & 290.61 & 2.28 & +84.9 & 244.9 & +82.3 & 258.1 & $\mathrm{~N}$ & 28.6 \\
\hline $12,4,39-41$ & 291.40 & 1.32 & +81.2 & 197.5 & +63.5 & 238.2 & N & 10.4 \\
\hline $12,4,110-112$ & 292.11 & 2.70 & +82.3 & 89.4 & +80.8 & 289.6 & $\mathrm{~N}$ & 8.0 \\
\hline $12,5,39-41$ & 292.90 & 2.21 & +83.9 & 110.8 & +77.8 & 219.3 & $\mathrm{~N}$ & 7.9 \\
\hline $12,5,110-112$ & 293.61 & .87 & +61.8 & 226.1 & -37.3 & 338.8 & $\mathrm{R}$ & 15.5 \\
\hline $12,6,32-34$ & 294.33 & 1.07 & +83.5 & 75.2 & -76.7 & 297.5 & $\mathrm{R}$ & 14.5 \\
\hline $12,6, i 18-120$ & 295.19 & .14 & -51.3 & 277.8 & -68.1 & 295.6 & $\mathrm{R}$ & \\
\hline $13,1,30-32$ & & 1.23 & .9 & 9 & & & $\mathrm{R}$ & 15.8 \\
\hline $13,1,90-92$ & 297.11 & .5 & +77.0 & 101.9 & -3 & 274.0 & $\mathrm{R}$ & 8.5 \\
\hline $13,2,30-32$ & 298.01 & .24 & +56.6 & 96.2 & -50.0 & 291.7 & $\mathrm{R}$ & 7.4 \\
\hline $13,2,90-92$ & 298.61 & .95 & +58.3 & 69.3 & -42.7 & 126.7 & $\mathrm{R}$ & 4.8 \\
\hline $13,3,30-32$ & 299.51 & .48 & +75.9 & 45.5 & +81.8 & 162.3 & $\mathrm{~N}$ & 10.2 \\
\hline $13,3,90-92$ & 300.11 & .88 & +70.5 & 52.2 & +86.8 & 142.5 & $\mathrm{~N}$ & 6.2 \\
\hline $13,4,30-32$ & 301.01 & .69 & +81.6 & 138.9 & +81.1 & 154.2 & $\mathrm{~N}$ & 5.0 \\
\hline $13,4,90-92$ & 301.61 & 1.51 & +60.8 & 76.4 & -68.0 & 271.3 & $\mathrm{R}$ & 4.8 \\
\hline $13,5,35-37$ & 302.56 & 7.13 & -40.5 & 165.6 & -69.3 & 281.4 & $\mathrm{R}$ & 60.8 \\
\hline $14,1,35-37$ & 306.16 & 50.86 & +72.5 & 43.7 & +71.9 & 29.3 & $\mathrm{~N}$ & 14.4 \\
\hline $14,1,103-105$ & 306.84 & 20.71 & +68.9 & 66.4 & +68.0 & 45.3 & $\mathrm{~N}$ & 25.5 \\
\hline $14,2,33-35$ & 307.64 & 34.99 & +68.9 & 12.4 .1 & +74.6 & 108.8 & $\mathrm{~N}$ & 28.1 \\
\hline $14,2,103-105$ & 308.34 & 5.54 & -15.2 & 198.9 & -69.6 & 275.5 & $\mathrm{R}$ & \\
\hline $5,1,19-2$ & 315.60 & 33.64 & +52.6 & 111.1 & -47.1 & 142.6 & $\mathrm{R}$ & 4.1 \\
\hline
\end{tabular}


Table A4. Paleomagnetic properties of samples from Hole 643A.

\begin{tabular}{|c|c|c|c|c|c|c|c|c|}
\hline $\begin{array}{l}\text { Core, Section, } \\
\text { Interval }(\mathrm{cm})\end{array}$ & $\begin{array}{l}\text { Depth } \\
\text { (mbsf) }\end{array}$ & $\begin{array}{c}\mathrm{J} \\
\mathrm{NRM} \\
(\mathrm{mA} / \mathrm{m})\end{array}$ & $\begin{array}{l}\text { Incl. } \\
\text { NRM } \\
\text { (deg) }\end{array}$ & $\begin{array}{l}\text { Decl. } \\
\text { NRM } \\
\text { (deg) }\end{array}$ & $\begin{array}{c}\text { Incl } \\
\text { stable } \\
\text { (deg) }\end{array}$ & $\begin{array}{l}\text { Decl. } \\
\text { stable } \\
\text { (deg) }\end{array}$ & Pol. & $\begin{array}{l}\text { MDF } \\
(\mathrm{mT})\end{array}$ \\
\hline $1,2,16-18$ & 1.67 & 1.77 & +69.2 & 134.4 & +73.5 & 136.9 & $\mathrm{~N}$ & 28.3 \\
\hline $1,2,60-62$ & 2.11 & 23.94 & +69.4 & 77.1 & +73.7 & 79.2 & $\mathrm{~N}$ & 22.7 \\
\hline $1,2,96-98$ & 2.47 & 76.53 & +85.6 & 113.1 & +85.8 & 93.2 & $\mathrm{~N}$ & 25.0 \\
\hline $1,2,130-132$ & 2.81 & 38.65 & +76.9 & 77.2 & +77.1 & 72.1 & $\mathrm{~N}$ & 29.9 \\
\hline $1,3,25-27$ & 3.26 & 9.86 & +63.1 & 179.4 & +69.0 & 72.9 & $\mathrm{~N}$ & 17.7 \\
\hline $1,3,60-62$ & 3.61 & 5.24 & +77.7 & 129.7 & +75.9 & 79.2 & $\mathrm{~N}$ & 27.4 \\
\hline $1,3,96-98$ & 3.97 & 26.80 & +77.4 & 104.1 & +81.2 & 94.1 & $\mathrm{~N}$ & 27.7 \\
\hline $1,3,135-137$ & 4.36 & 9.02 & +88.2 & 61.6 & +84.3 & 96.0 & $\mathrm{~N}$ & 27.9 \\
\hline $1,4,25-27$ & 4.76 & 2.77 & +86.8 & 118.2 & +70.8 & 86.8 & $\mathrm{~N}$ & 14.1 \\
\hline $2,1,55-57$ & 5.86 & 19.99 & +74.9 & 231.4 & +72.4 & 240.2 & $\mathrm{~N}$ & 23.9 \\
\hline $2,1,95-97$ & 6.26 & 1.27 & +57.3 & 265.3 & +68.8 & 263.9 & $\mathrm{~N}$ & 15.6 \\
\hline $2,1,135-137$ & 6.66 & 21.86 & +83.9 & 335.5 & +79.5 & 280.2 & $\mathrm{~N}$ & 14.5 \\
\hline $2,2,24-26$ & 7.05 & 66.12 & +89.2 & 233.5 & +88.6 & 228.1 & $\mathrm{~N}$ & 24.8 \\
\hline $2,2,55-57$ & 7.36 & 16.98 & +79.6 & 292.7 & +73.4 & 271.2 & $\mathrm{~N}$ & 14.8 \\
\hline $2,2,95-97$ & 7.76 & 80.69 & +80.6 & 236.0 & +80.3 & 247.3 & $\mathrm{~N}$ & 27.8 \\
\hline $2,2,135-137$ & 8.16 & 34.03 & +86.4 & 251.4 & +84.4 & 265.9 & $\mathrm{~N}$ & 29.0 \\
\hline $2,3,24-26$ & 8.55 & 29.19 & +77.5 & 311.1 & +75.6 & 259.4 & $\mathrm{~N}$ & 15.8 \\
\hline $2,3,55-57$ & 8.86 & 23.14 & +85.6 & 145.1 & +78.9 & 249.1 & $\mathrm{~N}$ & 20.2 \\
\hline $2,3,95-97$ & 9.26 & 9.68 & +61.2 & 252.7 & +79.9 & 265.5 & $\mathrm{~N}$ & 28.5 \\
\hline $2,3,135-137$ & 9.66 & 50.43 & +86.6 & 16.8 & +83.2 & 248.0 & $\mathrm{~N}$ & 21.8 \\
\hline $2,4,24-26$ & 10.05 & 107.81 & +84.8 & 294.8 & +84.0 & 272.7 & $\mathrm{~N}$ & 27.6 \\
\hline $2,4,55-57$ & 10.36 & 149.42 & +77.5 & 294.7 & +78.5 & 294.6 & $\mathrm{~N}$ & 27.4 \\
\hline $2,4,95-97$ & 10.76 & 1.04 & +63.6 & 200.0 & +76.0 & 287.4 & $\mathrm{~N}$ & 27.6 \\
\hline $2,4,135-137$ & 11.16 & 29.16 & +75.6 & 287.2 & +74.4 & 291.2 & $\mathrm{~N}$ & 28.4 \\
\hline $2,5,24-26$ & 11.55 & 37.97 & +82.1 & 278.6 & .8 & 285.9 & $\mathrm{~N}$ & 26.7 \\
\hline $2,5,55-57$ & 11.86 & 10.07 & +75.1 & 179.5 & .3 & 287.1 & $\mathrm{~N}$ & 16.4 \\
\hline $2,5,95-97$ & 12.26 & 41.14 & +70.8 & 284.7 & .1 & 280.2 & $\mathrm{~N}$ & 21.7 \\
\hline $2,5,135-137$ & 12.66 & 42.38 & +81.8 & 296.8 & .6 & 291.7 & $\mathrm{~N}$ & 21.3 \\
\hline $2,6,24-26$ & 13.05 & 59.01 & +86.5 & 255.7 & +84.0 & 270.8 & $\mathrm{~N}$ & 26.6 \\
\hline $2,6,55-57$ & 13.36 & 11.76 & +62.2 & 169.0 & -61.1 & 172.1 & & 9.2 \\
\hline $2,6,95-97$ & 13.76 & 5.95 & +81.0 & 203.8 & +81.2 & 267.6 & $\mathrm{~N}$ & 17.6 \\
\hline $2,6,135-137$ & 14.16 & .68 & +84.8 & 351.8 & +73.6 & 275.2 & $\mathrm{~N}$ & 9.7 \\
\hline $2,7,24-26$ & 14.55 & 10.33 & +81.9 & 258.3 & +80.8 & 271.8 & $\mathrm{~N}$ & 23.8 \\
\hline $3,1,29-31$ & 15.10 & 17.17 & +47.3 & & & & $\mathrm{~N}$ & 23.9 \\
\hline $3,2,25-27$ & 16.56 & 14.89 & +60.6 & 165.2 & +62.1 & 162.7 & $\mathrm{~N}$ & 21.5 \\
\hline $3,2,55-57$ & 16.86 & 19.12 & +78.1 & 139.3 & +64.6 & 158.4 & $\mathrm{~N}$ & 23.9 \\
\hline $3,2,95-97$ & 17.26 & 10.97 & +79.6 & 66.6 & +68.9 & 151.9 & $\mathrm{~N}$ & 20.0 \\
\hline $3,2,135-137$ & 17.66 & .68 & +55.5 & 179.2 & +76.1 & 152.1 & $\mathrm{~N}$ & 38.8 \\
\hline $3,3,25-27$ & 18.06 & 5.40 & +67.0 & 97.3 & +66.5 & 163.1 & $\mathrm{~N}$ & 32.8 \\
\hline $3,3,55-57$ & 18.36 & 1.45 & +69.4 & 203.6 & +75.7 & 157.8 & $\mathrm{~N}$ & 49.3 \\
\hline $3,3,95-97$ & 18.76 & 8.23 & +50.0 & 124.5 & +68.4 & 153.2 & $\mathrm{~N}$ & 23.0 \\
\hline $3,4,25-27$ & 19.56 & 3.26 & -3.2 & 194.9 & +81.5 & 143.6 & $\mathrm{~N}$ & 3.0 \\
\hline $3,4,55-57$ & 19.86 & 3.65 & +31.5 & 186.7 & +59.9 & 141.4 & $\mathrm{~N}$ & 26.2 \\
\hline $3,4,95-97$ & 20.26 & 6.79 & +38.4 & 30.4 & +64.5 & 142.0 & $\mathrm{~N}$ & 3.6 \\
\hline $3,4,135-137$ & 20.66 & 10.01 & +70.7 & 136.8 & +63.4 & 147.1 & $\mathrm{~N}$ & 7.1 \\
\hline $3,5,25-27$ & 21.06 & 3.67 & +50.0 & 118.9 & +65.5 & 144.7 & $\mathrm{~N}$ & 35.3 \\
\hline $3,5,55-57$ & 21.36 & 34.66 & +82.3 & 116.4 & +80.8 & 135.0 & $\mathrm{~N}$ & 5.1 \\
\hline
\end{tabular}


Table A4 (continued).

\begin{tabular}{|c|c|c|c|c|c|c|c|c|}
\hline $\begin{array}{l}\text { Core, Section, } \\
\text { Interval }(\mathrm{cm})\end{array}$ & $\begin{array}{l}\text { Depth } \\
\text { (mbsf) }\end{array}$ & $\begin{array}{c}J \\
\mathrm{NRM} \\
(\mathrm{mA} / \mathrm{m})\end{array}$ & $\begin{array}{l}\text { Incl. } \\
\text { NRM } \\
\text { (deg) }\end{array}$ & $\begin{array}{l}\text { Decl. } \\
\text { NRM } \\
\text { (deg) }\end{array}$ & $\begin{array}{c}\text { Incl } \\
\text { stable } \\
\text { (deg) }\end{array}$ & $\begin{array}{l}\text { Decl. } \\
\text { stable } \\
\text { (deg) }\end{array}$ & Pol. & $\begin{array}{l}\mathrm{MDF} \\
(\mathrm{mT})\end{array}$ \\
\hline $3,5,95-97$ & 21.76 & 16.20 & +60.4 & 146.0 & +61.3 & 146.2 & $\mathrm{~N}$ & 23.7 \\
\hline $3,5,135-137$ & 22.16 & 15.90 & +50.8 & 164.3 & +67.6 & 137.8 & $\mathrm{~N}$ & 19.0 \\
\hline $3,6,25-27$ & 22.56 & 8.37 & +69.1 & 143.6 & +83.8 & 134.0 & $\mathrm{~N}$ & 4.9 \\
\hline $3,6,55-57$ & 22.86 & 49.08 & +63.3 & 82.7 & +59.3 & 135.7 & $\mathrm{~N}$ & 4.4 \\
\hline $3,6,95-97$ & 23.26 & 1.28 & +2.1 & 168.3 & +70.1 & 141.5 & $\mathrm{~N}$ & 32.9 \\
\hline $3,6,135-137$ & 23.66 & 14.18 & +86.8 & 153.7 & +69.3 & 132.1 & $\mathrm{~N}$ & 3.0 \\
\hline $3,7,25-27$ & 24.06 & 33.87 & +66.6 & 107.1 & +65.6 & 135.7 & $\mathrm{~N}$ & 14.2 \\
\hline $3,7,54-56$ & 24.35 & 29.85 & +27.5 & 152.7 & +84.9 & 145.4 & $\mathrm{~N}$ & 38.9 \\
\hline $4,1,25-27$ & 24.56 & 19.08 & +72.3 & 199.4 & +74.6 & 206.1 & $\mathrm{~N}$ & 28.9 \\
\hline $4,1,55-57$ & 24.86 & 12.48 & +49.2 & 189.6 & +62.7 & 187.4 & $\mathrm{~N}$ & 5.7 \\
\hline $4,1,95-97$ & 25.26 & 6.44 & +75.0 & 194.5 & +84.3 & 184.5 & $\mathrm{~N}$ & 33.1 \\
\hline $4,1,135-137$ & 25.66 & 6.06 & +80.9 & 47.9 & +70.7 & 206.0 & $\mathrm{~N}$ & 32.1 \\
\hline $4,2,25-27$ & 26.06 & 3.46 & +36.5 & 193.3 & +80.0 & 182.5 & $\mathrm{~N}$ & 44.6 \\
\hline $4,2,55-57$ & 26.36 & 6.95 & +52.7 & 126.5 & +70.8 & 139.3 & $\mathrm{~N}$ & 39.9 \\
\hline $4,2,95-97$ & 26.76 & 39.91 & -79.9 & 353.8 & -79.2 & 355.4 & $\mathrm{R}$ & 30.7 \\
\hline $4,2,135-137$ & 27.16 & 57.08 & -83.3 & 351.9 & -81.9 & 351.1 & $\mathrm{R}$ & 30.5 \\
\hline $4,3,25-27$ & 27.56 & 26.49 & -68.5 & 30.1 & -70.2 & 22.5 & $\mathrm{R}$ & 30.2 \\
\hline $4,3,55-57$ & 27.86 & 31.89 & -82.6 & 39.5 & -83.9 & 18.9 & $\mathrm{R}$ & 27.5 \\
\hline $4,3,95-97$ & 28.26 & 6.46 & -75.5 & 267.3 & -80.5 & 1.8 & $\mathrm{R}$ & 39.5 \\
\hline $4,3,135-137$ & 28.66 & 20.73 & -81.6 & 41.3 & -79.7 & 9.0 & $\mathrm{R}$ & 28.8 \\
\hline $4,4,25-27$ & 29.06 & 50.60 & -78.6 & 20.4 & -77.8 & .6 & $\mathrm{R}$ & 28.9 \\
\hline $4,4,55-57$ & 29.36 & 42.55 & -61.9 & 308.7 & -78.9 & 355.7 & $\mathrm{R}$ & 26.2 \\
\hline $4,4,95-97$ & 29.76 & 36.70 & -75.0 & 59.9 & -78.8 & 47.4 & $\mathrm{R}$ & 30.6 \\
\hline $4,4,131-133$ & 30.12 & 27.48 & +84.0 & 211.8 & +85.3 & 186.1 & $\mathrm{~N}$ & 28.1 \\
\hline $4,5,25-27$ & 30.56 & 10.15 & +71.0 & 7.4 & +82.8 & 196.1 & $\mathrm{~N}$ & 18.4 \\
\hline $4,5,55-57$ & 30.86 & .85 & +76.0 & 218.8 & +71.4 & 206.7 & $\mathrm{~N}$ & 10.1 \\
\hline $4,5,95-97$ & 31.26 & 55.14 & +87.8 & 183.4 & +85.6 & 189.7 & $\mathrm{~N}$ & 23.4 \\
\hline $4,5,135-137$ & 31.66 & 22.39 & +78.7 & 190.9 & +83.2 & 185.2 & $\mathrm{~N}$ & 33.6 \\
\hline $4,6,25-27$ & 32.06 & 2.38 & -53.3 & 228.6 & +75.1 & 185.9 & $\mathrm{~N}$ & 16.0 \\
\hline $4,6,55-57$ & 32.36 & .32 & +64.0 & 144.9 & +80.3 & 144.5 & $\mathrm{~N}$ & 13.2 \\
\hline $4,6,95-97$ & 32.76 & 11.77 & -58.3 & 129.6 & -67.8 & 112.1 & $\mathrm{R}$ & 24.8 \\
\hline $4,6,135-137$ & 33.16 & 30.67 & -70.2 & 57.4 & -77.2 & 32.8 & $\mathrm{R}$ & 35.3 \\
\hline $4,7,25-27$ & 33.56 & 6.17 & +70.5 & 102.9 & -63.6 & 21.3 & $\mathrm{R}$ & 8.8 \\
\hline $4,7,55-57$ & 33.86 & 1.68 & +65.2 & 254.3 & -69.5 & 357.8 & $\mathrm{R}$ & 4.1 \\
\hline $5,1,25-27$ & 34.06 & 6.43 & +56.5 & 95.2 & +65.6 & 320.3 & & 12.7 \\
\hline $5,1,55-57$ & 34.36 & 16.52 & -66.3 & 140.2 & -76.3 & 153.6 & $\mathrm{R}$ & 36.4 \\
\hline $5,1,95-97$ & 34.76 & 10.44 & -58.6 & 137.1 & -68.4 & 140.3 & $\mathrm{R}$ & 23.0 \\
\hline $5,1,135-137$ & 35.16 & .82 & -45.5 & 102.3 & -64.0 & 116.9 & $\mathrm{R}$ & \\
\hline $5,2,25-27$ & 35.56 & 41.90 & -71.7 & 116.4 & -74.8 & 118.5 & $\mathrm{R}$ & 28.5 \\
\hline $5,2,55-57$ & 35.86 & 8.14 & -80.7 & 116.7 & -81.8 & 121.0 & $\mathrm{R}$ & 30.3 \\
\hline $5,2,95-97$ & 36.26 & 21.97 & -79.4 & 98.2 & -80.6 & 114.5 & $\mathrm{R}$ & 28.5 \\
\hline $5,2,135-137$ & 36.66 & 9.57 & -76.6 & 100.8 & -79.8 & 103.6 & $\mathrm{R}$ & 30.9 \\
\hline $5,3,25-27$ & 37.06 & 43.88 & -72.6 & 94.9 & -78.2 & 102.2 & $\mathrm{R}$ & 33.0 \\
\hline $5,3,55-57$ & 37.36 & 31.49 & -76.4 & 99.1 & -79.2 & 114.4 & $\mathrm{R}$ & 31.0 \\
\hline $5,3,95-97$ & 37.76 & 1.68 & -55.0 & 109.9 & -64.4 & 121.6 & $\mathrm{R}$ & 28.1 \\
\hline $5,3,135-137$ & 38.16 & 14.20 & -87.5 & 205.6 & -82.7 & 109.2 & $\mathrm{R}$ & 22.9 \\
\hline $5,4,25-27$ & 38.56 & 10.45 & -57.4 & 110.6 & -68.8 & 106.6 & $\mathrm{R}$ & 18.0 \\
\hline $5,4,55-57$ & 38.86 & 41.39 & -72.0 & 103.7 & -74.2 & 106.7 & $\mathrm{R}$ & 31.3 \\
\hline
\end{tabular}


Table A4 (continued).

\begin{tabular}{|c|c|c|c|c|c|c|c|c|}
\hline $\begin{array}{l}\text { Core, Section, } \\
\text { Interval (cm) }\end{array}$ & $\begin{array}{l}\text { Depth } \\
\text { (mbsf) }\end{array}$ & $\begin{array}{c}\mathrm{J} \\
\mathrm{NRM} \\
(\mathrm{mA} / \mathrm{m})\end{array}$ & $\begin{array}{c}\text { Incl. } \\
\text { NRM } \\
\text { (deg) }\end{array}$ & $\begin{array}{l}\text { Decl. } \\
\text { NRM } \\
\text { (deg) }\end{array}$ & $\begin{array}{c}\text { Incl } \\
\text { stable } \\
\text { (deg) }\end{array}$ & $\begin{array}{l}\text { Decl. } \\
\text { stable } \\
\text { (deg) }\end{array}$ & Pol. & $\begin{array}{l}\text { MDF } \\
(\mathrm{mT})\end{array}$ \\
\hline $5,4,95-97$ & 39.26 & 22.20 & -78.7 & 99.0 & -78.8 & 103.1 & $\mathrm{R}$ & 30.4 \\
\hline $5,4,135-137$ & 39.66 & .19 & -36.8 & 84.8 & -66.0 & 108.1 & $\mathrm{R}$ & \\
\hline $5,5,24-26$ & 40.05 & 36.30 & -78.8 & 112.2 & -81.9 & 113.6 & $\mathrm{R}$ & 26.0 \\
\hline $5,5,54-56$ & 40.35 & 8.65 & -80.4 & 91.3 & -80.4 & 110.1 & $\mathrm{R}$ & 32.7 \\
\hline $5,5,94-96$ & 40.75 & 12.22 & -78.6 & 224.6 & -69.9 & 115.7 & $\mathrm{R}$ & 22.5 \\
\hline $5,5,134-136$ & 41.15 & 31.01 & -84.4 & 119.5 & -83.2 & 124.2 & $\mathrm{R}$ & 28.5 \\
\hline $5,6,25-27$ & 41.56 & 17.24 & -85.9 & 127.6 & -83.5 & 125.7 & $\mathrm{R}$ & 25.8 \\
\hline $5, \varepsilon, 55-57$ & 41.86 & 17.63 & -86.0 & 159.0 & -82.7 & 118.4 & $\mathrm{R}$ & 32.4 \\
\hline $5,6,95-97$ & 42.26 & 14.81 & -82.7 & 156.4 & -74.9 & 114.7 & $\mathrm{R}$ & 29.6 \\
\hline $5,6,135-137$ & 42.66 & .09 & -86.3 & 54.8 & -84.0 & 111.8 & $\mathrm{R}$ & 23.5 \\
\hline $5,7, \quad 7-9$ & 42.88 & 3.57 & -84.6 & 190.6 & -80.7 & 108.3 & R & 34.5 \\
\hline $6,1,25-27$ & 43.56 & .14 & +25.5 & 124.4 & -64.0 & 72.6 & $\mathrm{R}$ & \\
\hline $6,1,54-56$ & 43.85 & .73 & -30.0 & 133.6 & -74.4 & 77.2 & $\mathrm{R}$ & \\
\hline $6,1,94-96$ & 44.25 & 23.90 & -63.8 & 106.3 & -68.1 & 81.1 & $\mathrm{R}$ & 37.7 \\
\hline $6,1,134-136$ & 44.65 & 17.91 & -56.3 & 94.6 & -67.7 & 97.7 & $\mathrm{R}$ & 36.7 \\
\hline $6,2,25-27$ & 45.06 & 15.13 & -74.4 & 37.7 & -76.8 & 70.0 & $\mathrm{R}$ & 31.9 \\
\hline $6,2,55-57$ & 45.36 & 17.10 & -89.2 & 305.3 & -82.4 & 70.6 & $\mathrm{R}$ & 35.1 \\
\hline $6,2,95-97$ & 45.76 & 1.66 & -20.6 & 13.7 & -65.2 & 99.4 & R & \\
\hline $6,2,135-137$ & 46.16 & .11 & -24.9 & 94.8 & -82.5 & 93.5 & R & \\
\hline $6,3,25-27$ & 46.56 & 13.39 & -68.6 & 99.1 & -75.3 & 99.9 & $\mathrm{R}$ & 32.3 \\
\hline $6,3,55-57$ & 46.86 & 48.05 & -83.2 & 118.3 & -85.5 & 94.5 & R & 28.6 \\
\hline $6,3,95-97$ & 47.26 & 11.04 & -80.3 & 19.2 & -84.9 & 105.0 & $\mathrm{R}$ & 29.1 \\
\hline $6,3,135-137$ & 47.66 & 44.50 & -60.0 & 113.6 & -69.5 & 104.2 & $\mathrm{R}$ & 29.5 \\
\hline $6,4,25-27$ & 48.06 & 37.85 & -63.4 & 114.6 & -75.8 & 109.6 & $\mathrm{R}$ & 24.3 \\
\hline $6,4,55-57$ & 48.36 & 33.22 & -53.8 & 89.4 & -73.3 & 85.6 & $\mathrm{R}$ & 36.1 \\
\hline $6,4,95-97$ & 48.76 & 3.11 & -61.8 & 89.5 & -72.9 & 87.4 & $\mathrm{R}$ & 14.7 \\
\hline $6,4,113-115$ & 48.94 & 5.50 & -44.2 & 89.4 & -61.4 & 75.4 & $\mathrm{R}$ & 16.3 \\
\hline $6,5,25-27$ & 49.56 & 36.38 & -58.5 & 79.3 & -63.8 & 80.1 & $\mathrm{R}$ & 24.5 \\
\hline $6,5,55-57$ & 49.86 & 13.37 & +58.8 & 110.8 & +81.3 & 211.3 & $\mathrm{~N}$ & 39.7 \\
\hline $6,5,95-97$ & 50.26 & 4.79 & -9.7 & 126.1 & +74.8 & 209.6 & $\mathrm{~N}$ & 47.1 \\
\hline $6,5,135-137$ & 50.66 & 4.53 & +17.8 & 85.6 & +85.5 & 211.2 & $\mathrm{~N}$ & 33.9 \\
\hline $6,6,25-27$ & 51.06 & 10.56 & +40.8 & 147.1 & +86.1 & 214.2 & $\mathrm{~N}$ & 48.3 \\
\hline $6,6,55-57$ & 51.36 & 47.71 & +81.3 & 120.8 & +78.5 & 201.6 & $\mathrm{~N}$ & 23.2 \\
\hline $5,6,95-97$ & 51.76 & 24.67 & +87.5 & 129.4 & +84.8 & 229.3 & $\mathrm{~N}$ & 35.8 \\
\hline $7,1,30-32$ & 53.11 & 41.98 & +77.1 & 62.0 & +73.1 & 79.8 & $\mathrm{~N}$ & 22.7 \\
\hline $7,1,72-74$ & 53.53 & 45.39 & +78.3 & 95.7 & +78.0 & 91.0 & $\mathrm{~N}$ & 30.5 \\
\hline $7,1,101-103$ & 53.82 & 31.65 & +71.7 & 78.2 & +71.8 & 76.5 & $\mathrm{~N}$ & 24.8 \\
\hline $7,1,133-135$ & 54.14 & 3.15 & +44.1 & 97.8 & +65.9 & 78.3 & $\mathrm{~N}$ & 46.2 \\
\hline $7,2,23-25$ & 54.54 & 7.39 & -87.7 & 281.4 & -78.1 & 245.2 & $\mathrm{R}$ & 25.9 \\
\hline $7,2,59-61$ & 54.90 & 1.29 & -56.1 & 176.7 & -73.4 & 221.4 & $\mathrm{R}$ & \\
\hline $7,2,100-102$ & 55.31 & 13.20 & -87.3 & 161.5 & -83.1 & 239.5 & $\mathrm{R}$ & 34.1 \\
\hline $7,2,132-134$ & 55.63 & 7.96 & -69.5 & 128.5 & -78.4 & 178.1 & $\mathrm{R}$ & 9.9 \\
\hline $7,3,25-27$ & 56.06 & 2.06 & -17.6 & 133.3 & -62.7 & 249.5 & $\mathrm{R}$ & \\
\hline $7,3,57-59$ & 56.38 & 32.95 & -74.1 & 249.1 & -71.8 & 267.6 & $\mathrm{R}$ & 33.2 \\
\hline $7,3,99-101$ & 56.80 & 17.96 & -73.7 & 257.5 & -70.3 & 289.3 & $\mathrm{R}$ & 46.4 \\
\hline $7,3,132-134$ & 57.13 & 24.36 & -80.4 & 334.4 & -75.2 & 299.1 & $\mathrm{R}$ & 35.8 \\
\hline $7,4,23-25$ & 57.54 & 24.81 & -63.0 & 307.6 & -72.1 & $3 ? 8.7$ & $\mathrm{R}$ & 40.4 \\
\hline $7,4,58-60$ & 57.89 & 30.39 & -71.7 & 344.9 & -66.6 & 341.5 & $\mathrm{R}$ & 34.5 \\
\hline
\end{tabular}




\begin{tabular}{|c|c|c|c|c|c|c|c|c|}
\hline $\begin{array}{l}\text { Core, Section, } \\
\text { Interval }(\mathrm{cm})\end{array}$ & $\begin{array}{l}\text { Depth } \\
\text { (mbsf) }\end{array}$ & $\begin{array}{c}\mathrm{J} \\
\mathrm{NRM} \\
(\mathrm{mA} / \mathrm{m})\end{array}$ & $\begin{array}{l}\text { Incl. } \\
\text { NRM } \\
\text { (deg) }\end{array}$ & $\begin{array}{l}\text { Decl. } \\
\text { NRM } \\
\text { (deg) }\end{array}$ & $\begin{array}{l}\text { Incl } \\
\text { stable } \\
\text { (deg) }\end{array}$ & $\begin{array}{l}\text { Decl. } \\
\text { stable } \\
\text { (deg) }\end{array}$ & Pol. & $\begin{array}{l}\text { MDF } \\
(\mathrm{mT})\end{array}$ \\
\hline $7,4,94-96$ & 58.25 & 26.13 & -61.8 & 22.8 & -65.9 & 15.9 & $\mathrm{R}$ & 26.7 \\
\hline $7,4,128-130$ & 58.59 & 6.24 & +13.8 & 159.5 & -65.5 & 220.7 & $\mathrm{R}$ & 23.6 \\
\hline $7,5,24-26$ & 59.05 & 27.43 & +53.5 & 108.2 & +56.8 & 117.0 & $\mathrm{~N}$ & 27.0 \\
\hline $7,5,56-58$ & 59.37 & 25.35 & +.3 & 163.5 & -1.3 & 169.4 & & 36.9 \\
\hline $7,5,94-96$ & 59.75 & 4.65 & -55.4 & 191.1 & -65.6 & 224.0 & $\mathrm{R}$ & \\
\hline $7,5,126-128$ & 60.07 & 3.01 & -36.1 & 94.4 & -68.4 & 275.5 & $R$ & \\
\hline $7,6,19-21$ & 60.50 & 1.94 & +3.4 & 88.4 & -82.2 & 279.4 & $\mathrm{R}$ & 38.5 \\
\hline $8,1,40-42$ & 62.71 & 2.11 & -6.2 & 61.6 & -65.6 & 352.7 & $\mathrm{R}$ & \\
\hline $8,1,71-73$ & 63.02 & 2.04 & -44.3 & 105.3 & -65.9 & 120.0 & $\mathrm{R}$ & 21.7 \\
\hline $8,1,106-108$ & 63.37 & 7.99 & +71.0 & 159.6 & +73.6 & 165.0 & $\mathrm{~N}$ & 22.6 \\
\hline $8,1,137-139$ & 63.68 & 1.11 & +71.5 & 193.7 & -54.2 & 215.1 & $\mathrm{R}$ & 17.1 \\
\hline $8,2,24-26$ & 64.05 & 2.88 & +61.1 & 250.4 & -73.1 & 269.1 & $\mathrm{R}$ & 8.8 \\
\hline $8,2,55-57$ & 64.36 & 5.18 & +68.2 & 193.4 & +72.7 & 193.7 & $\mathrm{~N}$ & 33.7 \\
\hline $8,2,89-91$ & 64.70 & .45 & +65.3 & 310.9 & +73.6 & 214.3 & $\mathrm{~N}$ & \\
\hline $8,2,130-132$ & 65.11 & 2.55 & +70.7 & 44.5 & +71.5 & 352.4 & $\mathrm{~N}$ & 16.7 \\
\hline $8,3,24-26$ & 65.55 & 6.46 & -72.7 & 75.8 & -76.7 & 67.3 & $\mathrm{R}$ & 24.6 \\
\hline $8,3,57-59$ & 65.88 & .47 & -31.0 & 77.7 & -67.3 & 42.2 & $\mathrm{R}$ & 60.9 \\
\hline $8,3,94-96$ & 66.25 & 17.45 & -44.7 & 61.9 & -63.1 & 48.0 & $\mathrm{R}$ & 28.5 \\
\hline $8,3,131-133$ & 66.62 & 14.02 & -63.0 & 52.6 & -68.0 & 35.2 & $\mathrm{R}$ & 24.3 \\
\hline $8,4,24-26$ & 67.05 & .90 & -48.9 & 78.5 & -64.8 & 45.8 & $\mathrm{R}$ & 66.8 \\
\hline $8,4,57-59$ & 67.38 & 6.17 & -26.8 & 83.0 & -68.3 & 55.9 & $\mathrm{R}$ & 12.1 \\
\hline $8,4,93-95$ & 67.74 & .30 & -58.4 & 95.9 & -70.3 & 50.4 & $\mathrm{R}$ & \\
\hline $8,4,131-133$ & 68.12 & .09 & -47.2 & 93.9 & -79.4 & 38.0 & $\mathrm{R}$ & 14.2 \\
\hline $8,5,24-26$ & 68.55 & .17 & +65.6 & 70.2 & +64.6 & 62.6 & $\mathrm{~N}$ & 47.8 \\
\hline $8,5,58-60$ & 68.89 & .26 & +70.4 & 115.2 & +65.3 & 181.8 & $\mathrm{~N}$ & 12.8 \\
\hline $8,5,93-95$ & 69.24 & .09 & -1.9 & 111.7 & +58.2 & 175.2 & $\mathrm{~N}$ & 32.8 \\
\hline $8,5,131-133$ & 69.62 & .08 & +32.4 & 120.2 & +57.6 & 189.1 & $\mathrm{~N}$ & 25.4 \\
\hline $8,6,24-26$ & 70.05 & 22.56 & +76.0 & 197.6 & +76.8 & 205.1 & $\mathrm{~N}$ & 38.6 \\
\hline $8,6,57-59$ & 70.38 & 2.26 & +7.4 & 118.4 & +62.1 & 155.2 & $\mathrm{~N}$ & 36.4 \\
\hline $8,6,92-94$ & 70.73 & 10.65 & -63.9 & 111.9 & -65.9 & 108.8 & & 34.9 \\
\hline $8,6,132-134$ & 71.13 & 28.03 & +73.7 & 215.8 & +71.7 & 220.0 & $\mathrm{~N}$ & 30.7 \\
\hline $9,1,25-27$ & 72.06 & 15.00 & +77.0 & 119.3 & +74.6 & 104.7 & $\mathrm{~N}$ & 29.0 \\
\hline $9,1,55-57$ & 72.36 & 13.23 & +66.1 & 108.7 & +71.0 & 99.2 & $\mathrm{~N}$ & 40.7 \\
\hline $9,1,95-97$ & 72.76 & 23.50 & +82.3 & 99.4 & +74.3 & 98.2 & $\mathrm{~N}$ & 36.6 \\
\hline $9,1,135-137$ & 73.16 & 12.88 & +73.3 & 82.8 & +73.0 & 83.7 & $\mathrm{~N}$ & 36.0 \\
\hline $9,2,20-22$ & 73.51 & 20.02 & +36.7 & 85.7 & +36.4 & 85.3 & $\mathrm{~N}$ & 39.1 \\
\hline $9,2,55-57$ & 73.86 & 19.95 & +75.9 & 133.0 & +75.2 & 96.8 & $\mathrm{~N}$ & 34.3 \\
\hline $9,2,95-97$ & 74.26 & 13.27 & +73.4 & 110.9 & +73.9 & 110.1 & $\mathrm{~N}$ & 36.6 \\
\hline $9,2,135-137$ & 74.66 & 17.03 & +70.4 & 115.2 & +67.4 & 114.2 & $\mathrm{~N}$ & 44.8 \\
\hline $9,3,25-27$ & 75.06 & 16.46 & +70.5 & 60.0 & +66.6 & 69.0 & $\mathrm{~N}$ & 34.5 \\
\hline $9,3,55-57$ & 75.36 & 20.75 & +65.7 & 72.5 & +67.0 & 67.8 & $\mathrm{~N}$ & 27.7 \\
\hline $9,3,95-97$ & 75.76 & 12.11 & +58.2 & 72.7 & +64.9 & 70.9 & $\mathrm{~N}$ & 35.0 \\
\hline $9,3,135-137$ & 76.16 & 20.59 & +70.9 & 61.2 & +70.6 & 62.6 & $\mathrm{~N}$ & 30.3 \\
\hline $9,4,25-27$ & 76.56 & 17.30 & +71.0 & 86.4 & +69.5 & 86.0 & $\mathrm{~N}$ & 33.0 \\
\hline $9,4,55-57$ & 76.86 & 12.26 & +74.2 & 10.8 & +71.7 & 67.3 & $\mathrm{~N}$ & 28.3 \\
\hline $9,4,95-97$ & 77.26 & 15.26 & +76.5 & 58.5 & +74.0 & 63.8 & $\mathrm{~N}$ & 31.6 \\
\hline $9,4,135-137$ & 77.66 & 8.80 & +74.1 & 84.8 & +72.5 & 69.5 & $\mathrm{~N}$ & 30.1 \\
\hline $9,5,25-27$ & 78.06 & 1.52 & +33.4 & 25.3 & -73.8 & 229.0 & $\mathrm{R}$ & 3.4 \\
\hline
\end{tabular}


Table A4 (continued).

\begin{tabular}{|c|c|c|c|c|c|c|c|c|}
\hline $\begin{array}{l}\text { Core, Section, } \\
\text { Interval }(\mathrm{cm})\end{array}$ & $\begin{array}{l}\text { Depth } \\
\text { (mbsf) }\end{array}$ & 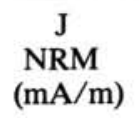 & $\begin{array}{l}\text { Incl. } \\
\text { NRM } \\
\text { (deg) }\end{array}$ & $\begin{array}{l}\text { Decl. } \\
\text { NRM } \\
\text { (deg) }\end{array}$ & $\begin{array}{l}\text { Incl } \\
\text { stable } \\
\text { (deg) }\end{array}$ & $\begin{array}{l}\text { Decl. } \\
\text { stable } \\
\text { (deg) }\end{array}$ & Pol. & $\begin{array}{l}\mathrm{MDF} \\
(\mathrm{mT})\end{array}$ \\
\hline $9,5,55-57$ & 78.36 & 10.39 & -75.4 & 252.4 & -73.3 & 249.6 & $\mathrm{R}$ & 36.0 \\
\hline $9,5,95-97$ & 78.76 & 1.96 & -73.6 & 168.5 & -69.3 & 247.5 & $\mathrm{R}$ & \\
\hline $9,6,25-27$ & 79.56 & 3.03 & +62.8 & 100.1 & -63.6 & 222.7 & $\mathrm{R}$ & 8.8 \\
\hline $9,6,57-59$ & 79.88 & .76 & -14.3 & 109.3 & -80.2 & 147.0 & $\mathrm{R}$ & 4.4 \\
\hline $9,6,95-97$ & 80.26 & 5.61 & +56.9 & 5.4 & +63.4 & 26.3 & & 31.2 \\
\hline $9,6,135-137$ & 80.66 & 1.52 & +37.2 & 97.6 & -66.9 & 110.7 & $\mathrm{R}$ & 5.3 \\
\hline $9,7,25-27$ & 81.06 & 2.92 & +62.4 & 162.5 & -72.6 & 143.2 & $\mathrm{R}$ & 7.3 \\
\hline $10,1,55-57$ & 81.86 & 2.59 & -56.6 & 188.2 & -69.2 & 201.4 & $\mathrm{R}$ & 47.2 \\
\hline $10,1,95-97$ & 82.26 & 4.22 & -73.7 & 166.8 & -78.3 & 192.1 & $\mathrm{R}$ & 38.3 \\
\hline $10,1,135-137$ & 82.66 & 9.33 & -64.8 & 202.0 & -68.9 & 198.0 & $\mathrm{R}$ & 36.5 \\
\hline $10,2,25-27$ & 83.06 & .56 & +73.9 & 74.5 & +67.8 & 22.6 & $\mathrm{~N}$ & 7.5 \\
\hline $10,2,55-57$ & 83.36 & 1.30 & +87.6 & 15.8 & +73.4 & 26.2 & $\mathrm{~N}$ & 9.7 \\
\hline $10,2,95-97$ & 83.76 & 3.77 & +70.1 & 49.0 & +67.4 & 22.4 & $\mathrm{~N}$ & 25.2 \\
\hline $10,2,135-137$ & 84.16 & 4.75 & +75.4 & 86.0 & +73.6 & 27.9 & $\mathrm{~N}$ & 22.2 \\
\hline $10,3,25-27$ & 84.56 & 12.19 & +67.6 & 12.9 & +67.4 & 10.1 & $\mathrm{~N}$ & 32.5 \\
\hline $10,3,95-97$ & 85.26 & 4.79 & +77.9 & 52.3 & +70.6 & 35.8 & $\mathrm{~N}$ & 28.5 \\
\hline $10,3,135-137$ & 85.66 & 2.35 & +7.5 & 228.5 & -21.4 & 231.9 & & 34.3 \\
\hline $10,4,25-27$ & 86.06 & 1.35 & +70.3 & 333.7 & +66.2 & .4 & $\mathrm{~N}$ & 8.2 \\
\hline $10,4,55-57$ & 86.36 & 1.06 & +67.6 & 330.2 & +73.7 & 328.5 & $\mathrm{~N}$ & 47.5 \\
\hline $10,4,95-97$ & 86.76 & .38 & +69.8 & 335.7 & +74.0 & 344.3 & $\mathrm{~N}$ & 8.6 \\
\hline $10,4,135-137$ & 87.16 & 3.34 & +22.9 & 345.8 & +77.1 & 358.9 & $\mathrm{~N}$ & 38.9 \\
\hline $10,5,25-27$ & 87.56 & .12 & +42.8 & 109.8 & +70.6 & 29.4 & $\mathrm{~N}$ & 11.3 \\
\hline $10,5,55-57$ & 87.86 & .11 & +70.3 & 30.6 & +73.7 & 11.0 & $\mathrm{~N}$ & 8.1 \\
\hline $10,5,95-97$ & 88.26 & .08 & +65.7 & 29.2 & +65.7 & 34.0 & $\mathrm{~N}$ & \\
\hline $10,6,25-27$ & 89.06 & .04 & +63.4 & 36.1 & +73.0 & 25.0 & $\mathrm{~N}$ & \\
\hline $10,6,55-57$ & 89.36 & .12 & +75.8 & 6.8 & +74.3 & 20.9 & $\mathrm{~N}$ & 5.8 \\
\hline $10,6,95-97$ & 89.76 & .15 & +65.3 & 13.0 & $+63 . \varepsilon$ & 20.7 & $\mathrm{~N}$ & 25.3 \\
\hline $10,6,135-137$ & 90.16 & .19 & +69.9 & 83.6 & +74.4 & 10.1 & $\mathrm{~N}$ & 27.4 \\
\hline $10,7,25-27$ & 90.56 & .27 & +74.6 & 73.1 & +66.5 & 22.9 & $\mathrm{~N}$ & 32.4 \\
\hline $11,1,25-27$ & 91.06 & .29 & +60.6 & 97.0 & -65.6 & 204.8 & $\mathrm{R}$ & 4.8 \\
\hline $11,1,55-57$ & 91.36 & .09 & +68.1 & 175.4 & -62.2 & 190.5 & $\mathrm{R}$ & 8.2 \\
\hline $11,1,95-97$ & 91.76 & .20 & -66.7 & 203.9 & -64.8 & 206.2 & $\mathrm{R}$ & 13.1 \\
\hline $11,1,135-137$ & 92.16 & .35 & -63.9 & 222.6 & -68.5 & 208.2 & $\mathrm{R}$ & 30.4 \\
\hline $11,2,25-27$ & 92.56 & .37 & +60.9 & 334.2 & -69.4 & 268.7 & $\mathrm{R}$ & 3.9 \\
\hline $11,2,55-57$ & 92.86 & .30 & -76.0 & 304.9 & -68.6 & 275.6 & $\mathrm{R}$ & 6.4 \\
\hline $11,2,95-97$ & 93.26 & .16 & -14.3 & 14.3 & -71.5 & 338.4 & $\mathrm{R}$ & 4.6 \\
\hline $11,2,135-137$ & 93.66 & .64 & +80.6 & 280.5 & +74.5 & 221.0 & $\mathrm{~N}$ & 14.0 \\
\hline $11,3,25-27$ & 94.06 & .93 & +36.9 & 245.1 & +72.5 & 223.0 & $\mathrm{~N}$ & 15.0 \\
\hline $11,3,55-57$ & 94.36 & .18 & +57.5 & 228.5 & +62.3 & 231.7 & $\mathrm{~N}$ & 8.0 \\
\hline $11,3,95-97$ & 94.76 & .24 & -39.9 & 151.7 & -74.7 & 122.5 & $\mathrm{~N}$ & 29.8 \\
\hline $11,3,135-137$ & 95.16 & .08 & +67.7 & 318.8 & +81.6 & 231.1 & $\mathrm{~N}$ & 9.8 \\
\hline $11,4,25-27$ & 95.56 & .18 & +71.4 & 252.4 & +59.2 & 250.0 & $\mathrm{~N}$ & 23.5 \\
\hline $11,4,55-57$ & 95.86 & .97 & -46.6 & 113.3 & -75.4 & 21.1 & $\mathrm{R}$ & \\
\hline $11,4,95-97$ & 96.26 & .42 & -41.1 & 104.9 & -81.8 & 42.5 & $\mathrm{R}$ & 13.9 \\
\hline $11,4,135-137$ & 96.66 & .19 & +36.9 & 26.9 & -63.5 & 34.4 & $\mathrm{R}$ & \\
\hline $11,5,25-27$ & 97.06 & .83 & -51.7 & 36.2 & -68.0 & 55.9 & $\mathrm{R}$ & 7.7 \\
\hline $11,5,55-57$ & 97.36 & .45 & -25.9 & 79.5 & -63.1 & 30.6 & $\mathrm{R}$ & 14.2 \\
\hline $11,5,95-97$ & 97.76 & .10 & +66.8 & 166.8 & +65.1 & 193.2 & $\mathrm{~N}$ & 14.6 \\
\hline
\end{tabular}


Table A4 (continued).

\begin{tabular}{|c|c|c|c|c|c|c|c|c|}
\hline $\begin{array}{l}\text { Core, Section, } \\
\text { Interval }(\mathrm{cm})\end{array}$ & $\begin{array}{l}\text { Depth } \\
\text { (mbsf) }\end{array}$ & $\begin{array}{c}\mathrm{J} \\
\mathrm{NRM} \\
(\mathrm{mA} / \mathrm{m})\end{array}$ & $\begin{array}{l}\text { Incl. } \\
\text { NRM } \\
\text { (deg) }\end{array}$ & $\begin{array}{l}\text { Decl. } \\
\text { NRM } \\
\text { (deg) }\end{array}$ & $\begin{array}{c}\text { Incl } \\
\text { stable } \\
\text { (deg) }\end{array}$ & $\begin{array}{l}\text { Decl. } \\
\text { stable } \\
\text { (deg) }\end{array}$ & Pol. & $\begin{array}{l}\text { MDF } \\
(\mathrm{mT})\end{array}$ \\
\hline $11,5,135-137$ & 98.16 & .15 & +61.0 & 99.1 & +69.6 & 191.1 & $\mathrm{~N}$ & 24.7 \\
\hline $11,6,25-27$ & 98.56 & .16 & +84.6 & 80.8 & +79.2 & 174.1 & $\mathrm{~N}$ & 4.6 \\
\hline $11,6,55-57$ & 98.86 & .11 & -38.3 & 233.8 & -63.7 & 148.7 & $\mathrm{~N}$ & 7.7 \\
\hline $11,6,95-97$ & 99.26 & .10 & +56.4 & 127.2 & +59.3 & 116.8 & $\mathrm{~N}$ & 12.0 \\
\hline $11,6,135-137$ & 99.66 & .32 & +54.5 & 129.3 & +66.8 & 98.5 & $\mathrm{~N}$ & 12.2 \\
\hline $11,7,25-27$ & 100.06 & .81 & +27.6 & 98.2 & +61.4 & 83.0 & $N$ & 36.1 \\
\hline $12,1,25-27$ & 100.56 & .38 & +60.8 & 71.7 & +68.1 & 151.3 & $\mathrm{~N}$ & 9.2 \\
\hline $12,1,55-57$ & 100.86 & .20 & -57.7 & 46.2 & -61.4 & 348.9 & & \\
\hline $12,1,95-97$ & 101.26 & .14 & +72.2 & 91.8 & +80.8 & 126.9 & $\mathrm{~N}$ & \\
\hline $12,1,135-137$ & 101.66 & .16 & -37.2 & 63.2 & -67.2 & 45.2 & & 19.7 \\
\hline $12,2,25-27$ & 102.06 & .18 & +62.5 & 87.7 & +61.2 & 86.7 & $\mathrm{~N}$ & \\
\hline $12,2,55-57$ & 102.36 & .45 & +67.7 & 58.1 & +67.1 & 54.1 & $\mathrm{~N}$ & 29.8 \\
\hline $12,2,95-97$ & 102.76 & .26 & +48.4 & 75.8 & +67.1 & 49.4 & $\mathrm{~N}$ & 22.6 \\
\hline $12,2,135-137$ & 103.16 & .50 & +68.1 & 80.7 & +68.4 & 58.9 & $\mathrm{~N}$ & 19.1 \\
\hline $12,3,25-27$ & 103.56 & .25 & +67.8 & 71.7 & +63.4 & 68.7 & $\mathrm{~N}$ & \\
\hline $12,3,55-57$ & 103.86 & .14 & +53.3 & 49.6 & +67.9 & 62.0 & $\mathrm{~N}$ & 38.2 \\
\hline $12,3,95-97$ & 104.26 & .25 & +70.5 & 56.9 & +65.9 & 66.8 & $\mathrm{~N}$ & \\
\hline $12,3,135-137$ & 104.66 & .12 & -65.8 & 102.6 & -67.7 & 176.8 & & \\
\hline $12,4,25-27$ & 105.06 & .28 & +63.6 & 47.1 & +65.4 & 46.7 & $\mathrm{~N}$ & \\
\hline $12,4,55-57$ & 105.36 & .10 & +83.4 & 97.4 & + & 63.1 & $\mathrm{~N}$ & 6.9 \\
\hline $12,4,95-97$ & 105.76 & .54 & +68.3 & 100.9 & 5 & 63.0 & $\mathrm{~N}$ & 14.6 \\
\hline $12,4,135-137$ & 106.16 & .17 & +64.5 & 65.7 & 4 & 55.8 & $\mathrm{~N}$ & 34.9 \\
\hline $12,5,25-27$ & 106.56 & .29 & +61.6 & 73.0 & +60.8 & 58.0 & $\mathrm{~N}$ & 40.8 \\
\hline $12,5,55-57$ & 106.86 & .19 & +66.1 & 64.4 & +67.9 & 54.3 & $\mathrm{~N}$ & 61.2 \\
\hline $12,5,95-97$ & 107.26 & .27 & +72.9 & 64.1 & +70.1 & 62.6 & $\mathrm{~N}$ & 30.1 \\
\hline $12,6,25-27$ & 108.06 & .32 & +64.7 & 70.0 & +63.2 & 65.6 & $\mathrm{~N}$ & 37.4 \\
\hline $12,6,55-57$ & 108.36 & .14 & +54.1 & 63.2 & +63.4 & 50.9 & $\mathrm{~N}$ & 30.7 \\
\hline $12,6,95-97$ & 108.76 & .24 & +71.3 & 99.0 & +71.9 & 59.1 & $\mathrm{~N}$ & 28.8 \\
\hline $12,6,135-137$ & 109.16 & .41 & +70.9 & 37.3 & +67.4 & 53.8 & $\mathrm{~N}$ & 8.5 \\
\hline $12,7,26-28$ & 109.57 & .07 & -39.4 & 82.9 & -67.6 & 103.7 & & 23.8 \\
\hline $13,1,25-27$ & 11 & & & & & & $\mathrm{~N}$ & 2.8 \\
\hline $13,1,55-57$ & 110 & .2 & 0 & 7 & 0 & 0 & $\mathrm{~N}$ & 65.6 \\
\hline $13,1,95-97$ & 110.76 & .28 & +54.6 & 60.4 & +6 & 43.6 & $\mathrm{~N}$ & 17.8 \\
\hline $13,1,135-137$ & 111.16 & .23 & +48.8 & 37.0 & +59.1 & 25.4 & $\mathrm{~N}$ & 8.3 \\
\hline $13,2,25-27$ & 111.56 & .15 & +36.9 & 109.8 & +85.3 & 39.6 & $\mathrm{~N}$ & \\
\hline $13,2,54-56$ & 111.85 & .08 & +46.6 & 58.0 & +68.2 & 44.1 & $\mathrm{~N}$ & 31.2 \\
\hline $13,2,95-97$ & 112.26 & .26 & +57.8 & 129.5 & +76.6 & 64.6 & $\mathrm{~N}$ & 3.9 \\
\hline $13,2,135-137$ & 112.66 & .24 & -24.0 & 353.8 & +59.4 & 50.8 & $\mathrm{~N}$ & 20.2 \\
\hline $13,3,25-27$ & 113.06 & .31 & +43.9 & 83.1 & +63.2 & 55.9 & $\mathrm{~N}$ & 3.8 \\
\hline $13,3,55-57$ & 113.36 & .49 & +46.7 & 71.8 & +60.3 & 49.9 & $\mathrm{~N}$ & 4.1 \\
\hline $13,3,95-97$ & 113.76 & 1.18 & +59.9 & 1.7 & +76.4 & 26.3 & $\mathrm{~N}$ & 22.0 \\
\hline $13,3,135-137$ & 114.16 & .29 & +77.1 & 46.0 & +70.1 & 33.7 & $\mathrm{~N}$ & 21.2 \\
\hline $13,4,25-27$ & 114.56 & .09 & +8.2 & 59.7 & -21.4 & 344.6 & $\mathrm{R}$ & 11.9 \\
\hline $13,4,55-57$ & 114.86 & .05 & -70.3 & 183.4 & -60.4 & 170.9 & $\mathrm{R}$ & 3.0 \\
\hline $13,4,95-97$ & 115.26 & .07 & -61.9 & 233.8 & -61.5 & 224.0 & $\mathrm{R}$ & \\
\hline $13,4,135-137$ & 115.66 & .13 & +61.7 & 164.3 & +66.6 & 38.0 & N & 28.1 \\
\hline $13,5,25-27$ & 116.06 & .06 & -1.6 & 118.2 & +62.7 & 24.0 & $\mathrm{~N}$ & \\
\hline $13,5,55-57$ & 116.36 & .12 & -84.1 & 113.4 & -72.6 & 192.4 & $\mathrm{R}$ & \\
\hline
\end{tabular}


Table A4 (continued).

\begin{tabular}{|c|c|c|c|c|c|c|c|c|}
\hline $\begin{array}{l}\text { Core, Section, } \\
\text { Interval }(\mathrm{cm})\end{array}$ & $\begin{array}{l}\text { Depth } \\
\text { (mbsf) }\end{array}$ & $\underset{\substack{\mathrm{J} \\
\mathrm{NAM} / \mathrm{m})}}{ }$ & $\begin{array}{l}\text { Incl. } \\
\text { NRM } \\
\text { (deg) }\end{array}$ & $\begin{array}{l}\text { Decl. } \\
\text { NRM } \\
\text { (deg) }\end{array}$ & $\begin{array}{c}\text { Incl } \\
\text { stable } \\
\text { (deg) }\end{array}$ & $\begin{array}{l}\text { Decl. } \\
\text { stable } \\
\text { (deg) }\end{array}$ & Pol. & $\begin{array}{l}\text { MDF } \\
(\mathrm{mT})\end{array}$ \\
\hline $13,5,95-97$ & 116.76 & .22 & +23.3 & 101.4 & -66.5 & 1956 & $\mathrm{R}$ & 19.1 \\
\hline $13,6,25-27$ & 117.56 & .10 & +36.2 & 93.6 & -73.0 & 210.8 & $\mathrm{R}$ & 9.9 \\
\hline $13,6,55-57$ & 117.86 & .06 & -49.5 & 174.2 & -68.6 & 187.2 & $\mathrm{R}$ & 7.8 \\
\hline $13,6,95-97$ & 118.26 & .09 & -73.8 & 230.2 & -66.7 & 186.4 & $\mathrm{R}$ & \\
\hline $13,6,135-137$ & 118.66 & .08 & +13.1 & 89.4 & -61.0 & 158.3 & $\mathrm{R}$ & \\
\hline $13,7,25-27$ & 119.06 & .06 & -74.3 & 223.6 & -74.4 & 200.1 & $\mathrm{R}$ & \\
\hline $14,1,25-27$ & 119.56 & .06 & -7.2 & 55.0 & -51.2 & 66.3 & $\mathrm{R}$ & 9.7 \\
\hline $14,1,57-59$ & 119.88 & .21 & +30.6 & 42.7 & +59.3 & 53.0 & $\mathrm{~N}$ & 41.7 \\
\hline $14,1,83-85$ & 120.14 & .12 & +9.0 & 84.6 & +77.4 & 93.2 & $\mathrm{~N}$ & \\
\hline $14,1,135-137$ & 120.66 & .10 & +42.5 & 67.8 & +75.1 & 74.1 & $\mathrm{~N}$ & 9.9 \\
\hline $14,2,25-27$ & 121.06 & .51 & +75.2 & 32.6 & +77.7 & 127.1 & $\mathrm{~N}$ & 8.5 \\
\hline $14,2,55-57$ & 121.36 & .12 & -74.1 & 346.4 & -74.8 & 349.9 & $R$ & \\
\hline $14,2,95-97$ & 121.76 & .02 & -25.0 & 66.2 & -78.3 & 333.1 & $\mathrm{R}$ & \\
\hline $14,2,135-137$ & 122.16 & .09 & -14.1 & 332.1 & -66.4 & 342.0 & $\mathrm{R}$ & \\
\hline $14,3,25-27$ & 122.56 & .05 & -61.4 & 303.7 & -76.8 & 334.4 & $\mathrm{R}$ & \\
\hline $14,3,55-57$ & 122.86 & .29 & -71.3 & 306.2 & -70.3 & 304.2 & $\mathrm{R}$ & 69.4 \\
\hline $14,3,95-97$ & 123.26 & .10 & -30.0 & 283.6 & -63.4 & 316.7 & $\mathrm{R}$ & \\
\hline $14,3,135-137$ & 123.66 & .04 & -25.9 & 350.8 & -77.1 & 315.0 & $\mathrm{R}$ & \\
\hline $14,4,25-27$ & 124.06 & .13 & +73.3 & 67.7 & -65.6 & 20.8 & $\mathrm{R}$ & 5.8 \\
\hline $14,4,55-57$ & 124.36 & .07 & -52.6 & 98.9 & -79.0 & 27.9 & $\mathrm{R}$ & \\
\hline $14,4,95-97$ & 124.76 & .11 & -46.9 & 70.1 & -66.5 & 53.6 & $\mathrm{R}$ & \\
\hline $14,4,135-137$ & 125.16 & .19 & -73.1 & 31.3 & -71.6 & 21.3 & $\mathrm{R}$ & 46.4 \\
\hline $14,5,25-27$ & 125.56 & .05 & -7.5 & 60.5 & -70.0 & 12.7 & $\mathrm{R}$ & \\
\hline $14,5,55-57$ & 125.86 & .12 & -50.3 & 59.4 & -61.2 & 44.4 & $\mathrm{R}$ & 45.0 \\
\hline $14,5,95-97$ & 126.26 & .11 & +1.1 & 32.6 & -66.7 & 7.3 & $\mathrm{R}$ & \\
\hline $14,5,135-137$ & 126.66 & .16 & -61.9 & 96.6 & -71.4 & 16.4 & $\mathrm{R}$ & 17.9 \\
\hline $14,6,25-27$ & 127.06 & .16 & +42.3 & 98.2 & -71.2 & 13.5 & $\mathrm{R}$ & 12.8 \\
\hline $14,6,55-57$ & 127.36 & .18 & -69.0 & 327.8 & -67.5 & 4.9 & $\mathrm{R}$ & 48.8 \\
\hline $14,6,95-97$ & 127.76 & .09 & +12.0 & 101.8 & -74.9 & 39.3 & $\mathrm{R}$ & 14.7 \\
\hline $15,1,25-27$ & 129.06 & .02 & -28.9 & 176.8 & & .2 & $\mathrm{R}$ & \\
\hline $15,1,55-57$ & 129.36 & .07 & -78.8 & 251.8 & -80.6 & 261.8 & $R$ & \\
\hline $15,1,95-97$ & 129.76 & .01 & +15.4 & 177.2 & -62.3 & 254.8 & $\mathrm{R}$ & \\
\hline $15,1,135-137$ & 130.16 & .09 & -21.5 & 120.4 & -83.1 & 259.6 & $\mathrm{R}$ & \\
\hline $15,2,25-27$ & 130.56 & .05 & +53.4 & 225.1 & -79.1 & 239.5 & $\mathrm{R}$ & 9.7 \\
\hline $15,2,55-57$ & 130.86 & .02 & +23.5 & 98.5 & -54.2 & 250.1 & $\mathrm{R}$ & \\
\hline $15,2,95-97$ & 131.26 & .0 & -60.9 & 252.5 & -66.1 & 251.1 & $\mathrm{R}$ & \\
\hline $15,2,135-137$ & 131.66 & .08 & -62.1 & 218.7 & -64.9 & 236.8 & $R$ & 3.3 \\
\hline $15,3,25-27$ & 132.06 & .20 & -77.3 & 227.7 & -74.4 & 216.1 & $\mathrm{R}$ & 9.4 \\
\hline $15,3,55-57$ & 132.36 & .08 & -31.4 & 209.5 & -59.9 & 218.1 & $\mathrm{R}$ & 18.0 \\
\hline $15,3,95-97$ & 132.76 & .09 & +26.8 & 123.0 & -75.4 & 234.7 & $\mathrm{R}$ & 33.0 \\
\hline $15,3,135-137$ & 133.16 & .05 & +13.9 & 231.5 & -65.8 & 223.2 & $\mathrm{R}$ & 4.7 \\
\hline $15,4,25-27$ & 133.56 & .07 & -37.6 & 206.8 & -71.2 & 218.7 & $\mathrm{R}$ & 38.3 \\
\hline $15,4,55-57$ & 133.86 & .05 & +56.2 & 244.1 & -66.2 & 227.5 & $\mathrm{R}$ & 9.0 \\
\hline $15,4,95-97$ & 134.26 & .07 & -8.5 & 181.6 & -63.4 & 184.4 & $\mathrm{R}$ & \\
\hline $15,4,135-137$ & 134.66 & .10 & +80.7 & 123.3 & +72.5 & 121.3 & $\mathrm{~N}$ & 13.8 \\
\hline $15,5,25-27$ & 135.06 & .30 & +20.3 & 136.6 & +64.7 & 126.9 & $\mathrm{~N}$ & 32.6 \\
\hline $15,5,55-57$ & 135.36 & .40 & +52.7 & 107.7 & +67.6 & 106.8 & $\mathrm{~N}$ & \\
\hline $15,5,95-97$ & 135.76 & .84 & +86.8 & 272.0 & +70.3 & 129.5 & $\mathrm{~N}$ & 38.7 \\
\hline
\end{tabular}


Table A4 (continued).

\begin{tabular}{|c|c|c|c|c|c|c|c|c|}
\hline $\begin{array}{l}\text { Core, Section, } \\
\text { Interval }(\mathrm{cm})\end{array}$ & $\begin{array}{l}\text { Depth } \\
\text { (mbsf) }\end{array}$ & 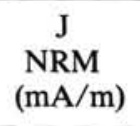 & $\begin{array}{l}\text { Incl. } \\
\text { NRM } \\
\text { (deg) }\end{array}$ & $\begin{array}{l}\text { Decl. } \\
\text { NRM } \\
\text { (deg) }\end{array}$ & $\begin{array}{c}\text { Incl } \\
\text { stable } \\
\text { (deg) }\end{array}$ & $\begin{array}{l}\text { Decl. } \\
\text { stable } \\
\text { (deg) }\end{array}$ & Pol. & $\begin{array}{l}\mathrm{MDF} \\
(\mathrm{mT})\end{array}$ \\
\hline $15,6,25-27$ & 136.56 & .50 & +63.7 & 114.6 & +65.0 & 114.4 & $\mathrm{~N}$ & 25.3 \\
\hline $15,6,55-57$ & 136.86 & .18 & +79.4 & 245.5 & +75.6 & 119.0 & $\mathrm{~N}$ & 14.4 \\
\hline $15,6,95-97$ & 137.26 & .12 & +61.9 & 321.2 & +70.2 & 125.7 & $\mathrm{~N}$ & \\
\hline $15,6,135-137$ & 137.66 & .28 & +79.2 & 113.0 & +73.9 & 107.5 & $\mathrm{~N}$ & 31.7 \\
\hline $15,7,25-27$ & 138.06 & .46 & +44.9 & 315.4 & +69.8 & 322.8 & $\mathrm{~N}$ & 36.0 \\
\hline $16,1,25-27$ & 138.56 & .16 & +40.9 & 169.3 & -59.0 & 168.3 & $\mathrm{R}$ & \\
\hline $16,1,55-57$ & 138.86 & .21 & +81.0 & 192.8 & -75.5 & 121.9 & $\mathrm{R}$ & 12.7 \\
\hline $16,1,95-97$ & 139.26 & .08 & +34.1 & 174.6 & -36.6 & 131.2 & $\mathrm{R}$ & \\
\hline $16,1,135-137$ & 139.66 & .19 & +47.2 & 356.9 & +48.5 & 40.9 & $\mathrm{~N}$ & 5.2 \\
\hline $16,2,25-27$ & 140.06 & .17 & +69.4 & 317.6 & +68.2 & 28.6 & $\mathrm{~N}$ & 4.7 \\
\hline $16,2,51-53$ & 140.32 & .35 & +73.2 & 322.5 & +75.3 & 20.4 & $\mathrm{~N}$ & 34.2 \\
\hline $16,2,95-97$ & 140.76 & .31 & +76.3 & 55.1 & +73.9 & 24.7 & $\mathrm{~N}$ & 24.0 \\
\hline $16,2,135-137$ & 141.16 & .25 & +74.4 & 324.1 & +68.6 & 5.2 & $\mathrm{~N}$ & 19.4 \\
\hline $16,3,25-27$ & 141.56 & .13 & +53.2 & 95.0 & +63.1 & 37.3 & $\mathrm{~N}$ & 19.9 \\
\hline $16,3,51-53$ & 141.82 & .09 & +71.6 & 64.8 & +71.4 & 27.6 & $\mathrm{~N}$ & 12.1 \\
\hline $16,3,95-97$ & 142.26 & .13 & +67.9 & 83.0 & +74.7 & 41.9 & $\mathrm{~N}$ & 22.0 \\
\hline $16,3,135-137$ & 142.66 & .15 & -15.8 & 73.1 & -51.9 & 66.2 & $\mathrm{R}$ & 15.4 \\
\hline $16,4,25-27$ & 143.06 & .19 & -43.5 & 97.9 & -79.9 & 198.6 & $\mathrm{R}$ & 14.5 \\
\hline $16,4,51-53$ & 143.32 & .03 & -38.0 & 113.5 & -75.9 & 213.3 & $\mathrm{R}$ & \\
\hline $16,4,95-97$ & 143.76 & .02 & +17.3 & 73.6 & -84.2 & 210.2 & $\mathrm{R}$ & \\
\hline $16,4,135-137$ & 144.16 & .26 & -50.4 & 89.2 & -76.5 & 141.0 & $\mathrm{R}$ & 13.1 \\
\hline $16,5,25-27$ & 144.56 & .11 & +61.3 & 99.4 & -71.0 & 160.8 & $\mathrm{R}$ & 5.8 \\
\hline $16,5,51-53$ & 144.82 & .03 & +42.8 & 13.5 & -63.9 & 164.3 & $\mathrm{R}$ & 24.0 \\
\hline $16,5,95-97$ & 145.26 & .06 & +44.9 & 94.0 & -50.0 & 189.7 & $\mathrm{R}$ & 5.5 \\
\hline $16,5,125-127$ & 145.56 & .27 & -36.7 & 257.3 & -72.8 & 197.5 & $\mathrm{R}$ & 18.7 \\
\hline $16,6,25-27$ & 146.06 & .16 & -51.2 & 256.1 & -80.1 & 216.4 & $\mathrm{R}$ & \\
\hline $16,6,51-53$ & 146.32 & .12 & -76.0 & 98.6 & -84.8 & 228.3 & $\mathrm{R}$ & \\
\hline $16,6,95-97$ & 146.76 & .09 & +17.5 & 281.6 & -60.3 & 226.1 & $\mathrm{R}$ & 6.6 \\
\hline $16,6,135-137$ & 147.16 & .81 & -55.1 & 252.9 & -80.1 & 239.8 & $\mathrm{R}$ & 20.1 \\
\hline $16,7,25-27$ & 147.56 & 1.33 & -47.3 & 264.1 & -72.1 & 259.9 & $\mathrm{R}$ & 17.6 \\
\hline $17,1,25-27$ & 148.06 & 1.55 & +35.0 & 98.7 & -41.4 & 98.0 & $\mathrm{R}$ & 25.8 \\
\hline $17,1,55-57$ & 148.36 & .66 & -32.2 & 116.2 & -65.7 & 97.7 & $\mathrm{R}$ & 14.7 \\
\hline $18,1,20-22$ & 157.51 & 7.10 & +69.8 & 246.0 & +68.4 & 302.0 & $\mathrm{~N}$ & 14.7 \\
\hline $18,1,67-69$ & 157.98 & .21 & +20.5 & 297.4 & +62.7 & 273.1 & $\mathrm{~N}$ & 13.7 \\
\hline $18,1,125-127$ & 158.56 & .11 & +82.7 & 336.1 & +75.7 & 308.9 & $N$ & 29.4 \\
\hline $18,2,20-22$ & 159.01 & .10 & +79.5 & 287.8 & +72.0 & 312.2 & $\mathrm{~N}$ & \\
\hline $18,2,67-69$ & 159.48 & .10 & -20.9 & 132.8 & -50.0 & 90.0 & $\mathrm{R}$ & 9.5 \\
\hline $18,2,125-127$ & 160.06 & 1.22 & -43.7 & 113.2 & -60.8 & 105.9 & $\mathrm{R}$ & 12.1 \\
\hline $18,3,20-22$ & 160.51 & 1.01 & -61.6 & 92.8 & -68.2 & 89.7 & $\mathrm{R}$ & 10.7 \\
\hline $19,1,25-27$ & 167.06 & .86 & -36.9 & 231.9 & -51.1 & 36.4 & $\mathrm{R}$ & 11.1 \\
\hline $19,1,55-57$ & 167.36 & .19 & -34.3 & 78.0 & -72.7 & 57.8 & $\mathrm{R}$ & 22.8 \\
\hline $19,1,97-99$ & 167.78 & .08 & -63.2 & 19.0 & -67.1 & 51.1 & $\mathrm{R}$ & 19.7 \\
\hline $19,1,135-137$ & 168.16 & .09 & -42.2 & 72.0 & -77.2 & 56.9 & $\mathrm{R}$ & \\
\hline $19,2,25-27$ & 168.56 & .07 & -37.0 & 90.9 & -70.7 & 51.8 & $\mathrm{R}$ & 6.4 \\
\hline $19,2,55-57$ & 168.86 & .03 & -1.5 & 46.7 & -58.4 & 357.0 & $\mathrm{R}$ & \\
\hline $19,2,97-99$ & 169.28 & .20 & -79.2 & 357.9 & -65.7 & 8.8 & $\mathrm{R}$ & 9.8 \\
\hline
\end{tabular}


Table A4 (continued).

\begin{tabular}{|c|c|c|c|c|c|c|c|c|}
\hline $\begin{array}{l}\text { Core, Section, } \\
\text { Interval }(\mathrm{cm})\end{array}$ & $\begin{array}{l}\text { Depth } \\
\text { (mbsf) }\end{array}$ & $\begin{array}{c}\mathrm{J} \\
\mathrm{NRM} \\
(\mathrm{mA} / \mathrm{m})\end{array}$ & $\begin{array}{l}\text { Incl. } \\
\text { NRM } \\
\text { (deg) }\end{array}$ & $\begin{array}{l}\text { Decl. } \\
\text { NRM } \\
\text { (deg) }\end{array}$ & $\begin{array}{l}\text { Incl } \\
\text { stable } \\
\text { (deg) }\end{array}$ & $\begin{array}{l}\text { Decl. } \\
\text { stable } \\
\text { (deg) }\end{array}$ & Pol. & $\begin{array}{l}\text { MDF } \\
(\mathrm{mT})\end{array}$ \\
\hline $9,2,135-137$ & 169.66 & .34 & -81.7 & 69.7 & -74.4 & 75.1 & $\mathrm{R}$ & 9.4 \\
\hline $19,3,25-27$ & 170.06 & .16 & +34.5 & 304.6 & +47.9 & 184.3 & $\mathrm{~N}$ & 22.6 \\
\hline $19,3,55-57$ & 170.36 & .14 & +34.4 & 333.3 & +69.0 & 171.8 & $\mathrm{~N}$ & 32.8 \\
\hline $19,3,97-99$ & 170.78 & .30 & -84.6 & 336.4 & -72.6 & 57.8 & $\mathrm{R}$ & 1.3 \\
\hline $19,3,135-137$ & 171.16 & .09 & +63.5 & 128.7 & -70.1 & 83.7 & $\mathrm{R}$ & 7.4 \\
\hline $19,4,25-27$ & 171.56 & .23 & +77.0 & 120.1 & -66.8 & 89.4 & $\mathrm{R}$ & 22.5 \\
\hline $19,4,55-57$ & 171.86 & .19 & -21.0 & 18.1 & -73.2 & 85.7 & $\mathrm{R}$ & 3.3 \\
\hline $19,4,97-99$ & 172.28 & .14 & -26.3 & 45.5 & -73.5 & 82.9 & $\mathrm{R}$ & 11.6 \\
\hline $19,4,135-137$ & 172.66 & 1.30 & -31.7 & 273.4 & -63.4 & 75.5 & $\mathrm{R}$ & 2.7 \\
\hline $19,5,25-27$ & 173.06 & .68 & +41.3 & 274.7 & -70.0 & 76.0 & $\mathrm{R}$ & 2.9 \\
\hline $19,5,55-57$ & 173.36 & .18 & -56.2 & 13.8 & -69.0 & 14.5 & $\mathrm{R}$ & 4.2 \\
\hline $19,5,97-99$ & 173.78 & .10 & -56.5 & 351.3 & -65.5 & 8.3 & $\mathrm{R}$ & \\
\hline $19,6,25-27$ & 174.56 & .05 & +52.5 & 32.9 & -72.9 & 3.8 & $\mathrm{R}$ & 49.1 \\
\hline $19,6,55-57$ & 174.86 & .05 & +63.2 & 287.8 & +65.9 & 180.4 & $\mathrm{R}$ & 9.6 \\
\hline $19,6,97-99$ & 175.28 & .03 & -51.2 & 305.8 & -85.8 & 342.4 & $\mathrm{R}$ & \\
\hline $19,6,135-137$ & 175.66 & .43 & -83.1 & 188.6 & -84.0 & 349.9 & $R$ & 12.7 \\
\hline $20,1,25-27$ & 17 & .30 & +66.9 & & -54 & 341 & $\mathrm{R}$ & 2.5 \\
\hline $20,1,55-57$ & 176.86 & .03 & +10.3 & 323.4 & -56.1 & 341.6 & $\mathrm{R}$ & \\
\hline $20,1,95-97$ & 177.26 & .28 & -86.5 & 207.1 & -79.5 & 345.8 & $\mathrm{R}$ & 25.1 \\
\hline $20,1,135-137$ & 177.66 & .22 & +72.7 & 70.2 & +73.3 & 64.0 & $\mathrm{~N}$ & 23.0 \\
\hline $20,2,25-27$ & 178.06 & .07 & +14.4 & 293.5 & +85.9 & 129.0 & $\mathrm{~N}$ & 41.4 \\
\hline $20,2,55-57$ & 178.36 & .09 & +79.7 & 166.2 & +72.9 & 141.1 & $\mathrm{~N}$ & 8.7 \\
\hline $20,2,95-97$ & 178.76 & .15 & +76.6 & 358.4 & +81.7 & 105.4 & $\mathrm{~N}$ & 8.6 \\
\hline $20,2,135-137$ & 179.16 & .62 & +70.7 & 126.0 & +62.7 & 124.5 & $\mathrm{~N}$ & 9.3 \\
\hline $20,3,25-27$ & 179.56 & .18 & +69.5 & 313.6 & +67.5 & 158.6 & $\mathrm{~N}$ & 20.8 \\
\hline $20,3,55-57$ & 179.86 & .16 & +75.6 & 142.1 & 8.5 & 125.8 & $\mathrm{~N}$ & 15.5 \\
\hline $20,3,95-97$ & 180.26 & .0 & +77.8 & 70.5 & 3.3 & 144.6 & $\mathrm{~N}$ & 17.5 \\
\hline $20,3,135-137$ & 180.66 & .04 & +69.9 & 174.0 & .7 & 132.5 & $\mathrm{~N}$ & 21.0 \\
\hline $20,4,25-27$ & 181.06 & .16 & +72.4 & 101.4 & +66.7 & 153.8 & $\mathrm{~N}$ & 3.9 \\
\hline $20,4,55-57$ & 181.36 & .22 & +58.6 & 116.0 & +62.5 & 142.1 & $\mathrm{~N}$ & 14.7 \\
\hline $20,4,35-97$ & 181.76 & .05 & +75.5 & 117.4 & +61.2 & 133.3 & $\mathrm{~N}$ & 12.5 \\
\hline $20,5,25-27$ & 182.56 & .18 & +80.5 & 40.4 & +72.9 & 151.5 & $\mathrm{~N}$ & 12.9 \\
\hline $20,5,55-57$ & 182.86 & .07 & -8.9 & 13.5 & -63.8 & 347.6 & $\mathrm{R}$ & \\
\hline $20,5,95-97$ & 183.26 & .21 & -82.2 & 61.9 & -78.8 & 306.5 & $\mathrm{R}$ & 19.7 \\
\hline $20,5,135-137$ & 183.66 & .07 & +46.5 & 12.0 & -88.6 & 270.0 & $\mathrm{R}$ & \\
\hline $20,6,25-27$ & 184.06 & .06 & +65.2 & 206.9 & -64.0 & 294.3 & $\mathrm{R}$ & 3.6 \\
\hline $20,6,55-57$ & 184.36 & .24 & -73.7 & 245.2 & -74.7 & 314.8 & $\mathrm{R}$ & 9.3 \\
\hline $20,6,95-97$ & 184.76 & .02 & +31.2 & 90.2 & -71.2 & 303.0 & $\mathrm{R}$ & \\
\hline $20,6,135-137$ & 185.16 & .19 & +63.5 & 316.0 & +74.4 & 220.9 & $\mathrm{~N}$ & 49.8 \\
\hline $20,7,25-27$ & 185.56 & .05 & +68.2 & 8.7 & +79.1 & 72.9 & $\mathrm{~N}$ & 35.7 \\
\hline $22,1,43-45$ & 195 & .1 & +72.2 & $35 \varepsilon$ & & & $\mathbf{N}$ & 31.7 \\
\hline $22,1,93-95$ & 196.24 & .2 & +79.1 & 68.4 & +78.0 & 318.2 & $\mathrm{~N}$ & 30.0 \\
\hline $22,1,144-146$ & 196.75 & .14 & +41.9 & 317.5 & +65.6 & 329.8 & $\mathrm{~N}$ & 37.0 \\
\hline $22,2,43-45$ & 197.24 & .15 & +71.2 & 32.4 & +71.2 & 323.5 & $\mathrm{~N}$ & 19.8 \\
\hline $22,2,93-95$ & 197.74 & .17 & +74.5 & 220.1 & +76.7 & 335.0 & $\mathrm{~N}$ & 24.5 \\
\hline $22,2,144-146$ & 198.25 & .60 & -21.9 & 331.0 & +67.7 & 318.7 & $\mathrm{~N}$ & 3.2 \\
\hline $22,3,43-45$ & 198.74 & .15 & +50.1 & 311.0 & +70.2 & 312.0 & $N$ & 29.8 \\
\hline 22,3 & 199.24 & .39 & 74.6 & 326.7 & +69.3 & 315.6 & $\mathrm{~N}$ & \\
\hline
\end{tabular}


Table A4 (continued).

\begin{tabular}{|c|c|c|c|c|c|c|c|c|}
\hline $\begin{array}{l}\text { Core, Section, } \\
\text { Interval }(\mathrm{cm})\end{array}$ & $\begin{array}{l}\text { Depth } \\
\text { (mbsf) }\end{array}$ & $\underset{(\mathrm{mA} / \mathrm{m})}{\stackrel{\mathrm{J}}{\mathrm{NRM}}}$ & $\begin{array}{l}\text { Incl. } \\
\text { NRM } \\
\text { (deg) }\end{array}$ & $\begin{array}{l}\text { Decl. } \\
\text { NRM } \\
\text { (deg) }\end{array}$ & $\begin{array}{c}\text { Incl } \\
\text { stable } \\
\text { (deg) }\end{array}$ & $\begin{array}{l}\text { Decl. } \\
\text { stable } \\
\text { (deg) }\end{array}$ & Pol. & $\begin{array}{l}\mathrm{MDF} \\
(\mathrm{mT})\end{array}$ \\
\hline $22,3,144-146$ & 199.75 & .14 & +78.7 & 227.0 & +73.4 & 324.3 & $\mathrm{~N}$ & 32.4 \\
\hline $22,4,43-45$ & 200.24 & .11 & +74.3 & 299.3 & +68.9 & 318.7 & $\mathrm{~N}$ & 39.3 \\
\hline $22,4,93-95$ & 200.74 & .16 & +78.9 & 70.0 & +66.7 & 326.2 & $\mathrm{~N}$ & 4.2 \\
\hline $22,4,131-133$ & 201.12 & .14 & +63.2 & 97.1 & +64.4 & 332.8 & $\mathrm{~N}$ & 3.1 \\
\hline $22,5,43-45$ & 201.74 & & & & +58.9 & 282.2 & $\mathrm{~N}$ & 99.9 \\
\hline $22,5,93-95$ & 202.24 & .07 & +83.6 & 347.0 & +72.8 & 341.8 & $\mathrm{~N}$ & 8.9 \\
\hline $22,5,144-146$ & 202.75 & .19 & +62.3 & 291.9 & +70.3 & 324.2 & $\mathrm{~N}$ & 4.0 \\
\hline $22,6,43-45$ & 203.24 & .08 & +86.2 & 164.1 & +63.8 & 340.3 & $\mathrm{~N}$ & 7.4 \\
\hline $22,6,93-95$ & 203.74 & .21 & +52.7 & 79.1 & +62.1 & 328.6 & $\mathrm{~N}$ & 3.8 \\
\hline $22,6,144-146$ & 204.25 & .22 & +45.0 & 38.1 & +67.2 & 327.0 & $\mathrm{~N}$ & 3.3 \\
\hline $22,7,24-26$ & 204.55 & .16 & +67.6 & 321.4 & +69.6 & 322.1 & $\mathrm{~N}$ & 7.0 \\
\hline $23,1,35-37$ & 205.46 & .13 & +50.8 & 271.3 & +64.7 & 266.4 & $\mathrm{~N}$ & 2.9 \\
\hline $23,1,85-87$ & 205.96 & .05 & +21.2 & 223.8 & +62.3 & 257.1 & $\mathrm{~N}$ & 6.9 \\
\hline $23,1,135-137$ & 206.46 & .05 & +49.5 & 245.0 & +56.2 & 260.8 & $\mathrm{~N}$ & 15.0 \\
\hline $23,2,35-37$ & 206.96 & .22 & +47.7 & 173.2 & +76.3 & 283.0 & $\mathrm{~N}$ & 3.1 \\
\hline $23,2,85-87$ & 207.46 & .09 & +65.4 & 269.5 & +68.3 & 287.0 & $\mathrm{~N}$ & 9.6 \\
\hline $23,2,135-137$ & 207.96 & .45 & +30.0 & 251.8 & +62.4 & 284.0 & $\mathrm{~N}$ & 3.4 \\
\hline $23,3,35-37$ & 208.46 & .17 & +16.4 & 86.0 & +77.5 & 330.3 & $\mathrm{~N}$ & 3.1 \\
\hline $23,3,85-87$ & 208.96 & .05 & +59.4 & 230.4 & +72.5 & 258.6 & $\mathrm{~N}$ & 8.9 \\
\hline $23,3,135-137$ & 209.46 & .11 & +73.7 & 184.8 & +82.3 & 286.6 & $\mathrm{~N}$ & 13.1 \\
\hline $23,4,35-37$ & 209.96 & .04 & +7.6 & 295.9 & +68.9 & 280.6 & $\mathrm{~N}$ & 12.3 \\
\hline $23,4,85-87$ & 210.46 & .02 & +60.0 & 26.7 & +70.1 & 283.8 & $\mathrm{~N}$ & \\
\hline $23,4,135-137$ & 210.96 & .06 & +61.3 & 318.3 & +70.6 & 292.8 & $\mathrm{~N}$ & 9.7 \\
\hline $23,5,35-37$ & 211.46 & .04 & +56.2 & 329.6 & -68.7 & 120.5 & $\mathrm{R}$ & \\
\hline $23,5,85-87$ & 211.96 & .05 & +51.0 & 38.6 & -61.9 & 115.6 & $\mathrm{R}$ & \\
\hline $23,6,35-37$ & 212.96 & .06 & -56.4 & 149.9 & -54.1 & 146.9 & $\mathrm{R}$ & 2.5 \\
\hline $23,6,85-87$ & 213.46 & .02 & +46.7 & 321.4 & +71.1 & 286.8 & & 4.1 \\
\hline $23,6,135-137$ & 213.96 & .07 & -57.2 & 251.5 & -76.5 & 124.2 & $\mathrm{R}$ & 13.3 \\
\hline $23,7,35-37$ & 214.46 & .13 & -62.1 & 92.5 & -77.6 & 113.4 & $\mathrm{R}$ & 12.0 \\
\hline $24,1,35-37$ & 215.26 & .04 & +84.7 & 204.2 & -75.9 & 299.0 & $\mathrm{R}$ & 4.2 \\
\hline $24,1,85-87$ & 215.76 & .04 & +65.6 & 277.2 & -72.3 & 303.7 & $\mathrm{R}$ & 9.0 \\
\hline $24,1,135-137$ & 216.26 & .14 & +37.0 & 125.5 & -49.3 & 319.4 & $\mathrm{R}$ & 3.0 \\
\hline $24,2,35-37$ & 216.76 & .07 & +65.8 & 169.5 & +71.6 & 167.0 & $\mathrm{~N}$ & 4.5 \\
\hline $24,2,85-87$ & 217.26 & .05 & +76.5 & 356.8 & +69.2 & 113.8 & $\mathrm{~N}$ & 8.9 \\
\hline $24,2,135-137$ & 217.76 & & & & +65.8 & 124.3 & $\mathrm{~N}$ & 5.6 \\
\hline $24,3,35-37$ & 218.26 & .11 & +68.7 & 128.0 & +67.1 & 117.4 & $\mathrm{~N}$ & 13.6 \\
\hline $24,3,85-87$ & 218.76 & .05 & +87.9 & 121.7 & +74.2 & 111.4 & $\mathrm{~N}$ & 12.7 \\
\hline $24,3,135-137$ & 219.26 & .04 & +60.6 & 131.1 & +64.4 & 123.7 & $\mathrm{~N}$ & 5.3 \\
\hline $24,4,35-37$ & 219.76 & .22 & +83.8 & 161.3 & +66.8 & 121.5 & $\mathrm{~N}$ & 5.4 \\
\hline $24,4,85-87$ & 220.26 & .06 & +33.8 & 319.5 & -62.8 & 334.0 & $\mathrm{R}$ & 25.9 \\
\hline $24,4,135-137$ & 220.76 & .04 & -58.6 & 320.9 & -60.0 & 323.9 & $\mathrm{R}$ & \\
\hline $24,5,35-37$ & 221.26 & .07 & +30.9 & 229.9 & -17.6 & 323.0 & $\mathrm{R}$ & 11.0 \\
\hline $24,5,85-87$ & 221.76 & .07 & +70.6 & 85.3 & +66.5 & 78.7 & $\mathrm{~N}$ & \\
\hline $24,5,135-137$ & 222.26 & .07 & +57.2 & 2.8 & +62.3 & 138.5 & $\mathrm{~N}$ & 9.4 \\
\hline $24,6,35-37$ & 222.76 & .07 & +61.7 & 237.8 & +80.7 & 146.2 & $\mathrm{~N}$ & 6.6 \\
\hline $24,6,85-87$ & 223.26 & .07 & +76.3 & 196.3 & +66.0 & 132.6 & $\mathrm{~N}$ & 8.0 \\
\hline $24,6,135-137$ & 223.76 & .04 & +17.1 & 73.6 & +55.6 & 104.6 & $\mathrm{~N}$ & 14.6 \\
\hline $24,7,22-24$ & 224.13 & .06 & +63.0 & 94.2 & +67.8 & 121.0 & $\mathrm{~N}$ & 2.0 \\
\hline
\end{tabular}


Table A4 (continued).

\begin{tabular}{|c|c|c|c|c|c|c|c|c|}
\hline $\begin{array}{l}\text { Core, Section, } \\
\text { Interval }(\mathrm{cm})\end{array}$ & $\begin{array}{l}\text { Depth } \\
\text { (mbsf) }\end{array}$ & 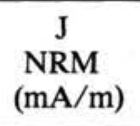 & $\begin{array}{l}\text { Incl. } \\
\text { NRM } \\
\text { (deg) }\end{array}$ & $\begin{array}{l}\text { Decl. } \\
\text { NRM } \\
\text { (deg) }\end{array}$ & $\begin{array}{c}\text { Incl } \\
\text { stable } \\
\text { (deg) }\end{array}$ & $\begin{array}{l}\text { Decl. } \\
\text { stable } \\
\text { (deg) }\end{array}$ & Pol. & $\begin{array}{l}\text { MDF } \\
(\mathrm{mT})\end{array}$ \\
\hline $25,1,35-37$ & 225.06 & .04 & +16.6 & 22.9 & -49.9 & 126.2 & $\mathrm{R}$ & \\
\hline $25,1,85-87$ & 225.56 & .02 & -1.4 & 144.8 & -54.7 & 177.4 & $\mathrm{R}$ & 12.2 \\
\hline $25,1,135-137$ & 226.06 & .03 & +67.5 & 316.3 & +60.0 & 324.6 & & 7.2 \\
\hline $25,2,35-37$ & 226.56 & .02 & +36.2 & 325.4 & -65.6 & 72.7 & $\mathrm{R}$ & 12.9 \\
\hline $25,2,85-87$ & 227.06 & .03 & -5.9 & 303.5 & -80.3 & 113.4 & $\mathrm{R}$ & 8.8 \\
\hline $25,2,135-137$ & 227.56 & .05 & +70.1 & 325.5 & +63.0 & 332.1 & $\mathrm{~N}$ & \\
\hline $25,3,35-37$ & 228.06 & .02 & -67.6 & 309.1 & +65.8 & 357.9 & $\mathrm{~N}$ & 2.8 \\
\hline $25,3,85-87$ & 228.56 & .05 & +70.1 & 330.0 & +62.4 & 341.9 & $\mathrm{~N}$ & 13.6 \\
\hline $25,3,135-137$ & 229.06 & .07 & +69.9 & 32.9 & +66.9 & 6.4 & $\mathrm{~N}$ & 14.2 \\
\hline $25,4,35-37$ & 229.56 & .03 & +77.1 & 325.7 & +74.6 & 342.0 & $\mathrm{~N}$ & \\
\hline $25,4,85-87$ & 230.06 & .06 & +63.6 & 343.4 & +60.5 & 355.3 & $\mathrm{~N}$ & 7.9 \\
\hline $25,4,135-137$ & 230.56 & .07 & +2.4 & 290.8 & +81.7 & 337.5 & $\mathrm{~N}$ & 4.1 \\
\hline $25,5,35-37$ & 231.06 & .08 & +3.1 & 284.5 & +77.5 & 338.1 & $\mathrm{~N}$ & 4.8 \\
\hline $25,5,85-87$ & 231.56 & .02 & +64.6 & 336.7 & +64.6 & 328.1 & $\mathrm{~N}$ & \\
\hline $25,5,135-137$ & 232.06 & .05 & +64.4 & 319.6 & +60.6 & 326.8 & $\mathrm{~N}$ & 13.3 \\
\hline $25,6,35-37$ & 232.56 & .08 & +67.5 & 344.1 & +68.6 & 344.9 & $\mathrm{~N}$ & 5.9 \\
\hline $25,6,85-87$ & 233.06 & .06 & +76.0 & 302.5 & +59.8 & 333.7 & $\mathrm{~N}$ & 4.4 \\
\hline $25,7,35-37$ & 234.06 & .02 & +74.2 & 328.2 & +65.7 & 343.3 & $\mathrm{~N}$ & 8.6 \\
\hline $26,1,35-37$ & 234.86 & .30 & -10.9 & 270.6 & -76.4 & 218.2 & $\mathrm{R}$ & 3.3 \\
\hline $26,1,85-87$ & 235.36 & .04 & +49.3 & 34.6 & -59.2 & 151.5 & $\mathrm{R}$ & \\
\hline $26,1,135-137$ & 235.86 & .02 & +44.6 & 71.8 & -41.6 & 152.0 & $\mathrm{R}$ & 18.2 \\
\hline $26,2,35-37$ & 236.36 & .04 & +67.4 & 25.9 & -56.5 & 201.5 & R & 14.4 \\
\hline $26,2,85-87$ & 236.86 & .07 & +77.7 & 22.4 & -82.1 & 141.3 & $\mathrm{R}$ & 3.6 \\
\hline $26,2,135-137$ & 237.36 & .04 & +36.0 & 312.3 & -78.5 & 203.1 & $\mathrm{R}$ & 7.6 \\
\hline $26,3,35-37$ & 237.86 & .05 & +37.0 & 290.7 & -64.3 & 243.2 & $\mathrm{R}$ & 3.4 \\
\hline $26,3,85-87$ & 238.36 & .03 & -68.6 & 208.8 & -66.0 & 236.6 & R & 4.4 \\
\hline $26,3,135-137$ & 238.86 & .06 & +63.9 & 188.7 & -85.6 & 209.9 & $\mathrm{R}$ & 3.1 \\
\hline $26,4,35-37$ & 239.36 & .08 & -77.4 & 193.6 & -85.6 & 208.5 & R & 19.3 \\
\hline $26,4,85-87$ & 239.86 & .04 & +39.6 & 110.8 & -53.4 & 52.3 & $\mathrm{R}$ & \\
\hline $26,5,35-37$ & 240.86 & .04 & +82.3 & 349.8 & +71.8 & 326.2 & $\mathrm{~N}$ & 9.0 \\
\hline $26,5,85-87$ & 241.36 & .04 & +77.3 & 343.6 & +79.3 & 353.0 & $\mathrm{~N}$ & 4.3 \\
\hline $26,5,135-137$ & 241.86 & .04 & +75.7 & 357.7 & +69.1 & 12.0 & $\mathrm{~N}$ & \\
\hline $26,6,35-37$ & 242.36 & .04 & +85.8 & 76.5 & $\div 60.4$ & 345.9 & $\mathrm{~N}$ & 14.4 \\
\hline $26,6,85-87$ & 242.86 & .11 & +77.3 & 43.6 & +76.7 & 354.5 & $\mathrm{~N}$ & 3.3 \\
\hline $26,6,135-137$ & 243.36 & .04 & +51.1 & 303.1 & +67.4 & 34.0 & $\mathrm{~N}$ & 4.9 \\
\hline $27,1,35-37$ & 244.66 & .02 & +84.8 & 35.4 & +61.5 & 68.5 & $\mathrm{~N}$ & \\
\hline $27,1,85-87$ & 245.16 & .03 & +30.7 & 130.4 & +75.5 & 54.7 & $\mathrm{~N}$ & 29.7 \\
\hline $27,1,135-137$ & 245.66 & .06 & -33.9 & 190.5 & +41.4 & 75.4 & $\mathrm{~N}$ & 18.3 \\
\hline $27,2,35-37$ & 246.16 & .02 & +73.2 & 324.3 & +57.6 & 331.6 & $\mathrm{~N}$ & \\
\hline $27,2,85-87$ & 246.66 & & +39.5 & 157.2 & +70.0 & 58.4 & $\mathrm{~N}$ & 10.3 \\
\hline $27,2,135-137$ & 247.16 & .02 & +4.9 & 298.6 & -80.0 & 329.7 & $\mathrm{R}$ & \\
\hline $27,3,35-37$ & 247.66 & .02 & +9.0 & 269.9 & -56.4 & 128.2 & $\mathrm{R}$ & \\
\hline $27,3,85-87$ & 248.16 & .02 & -48.5 & 140.9 & -50.5 & 87.4 & $\mathrm{R}$ & 3.4 \\
\hline $27,3,135-137$ & 248.66 & .09 & +16.5 & 180.0 & +2.3 & 184.6 & & 3.1 \\
\hline $27,4,29-31$ & 249.10 & .04 & +75.7 & 34.0 & +55.8 & 217.1 & $\mathrm{~N}$ & 9.7 \\
\hline $27,4,85-87$ & 249.66 & .03 & +76.7 & 110.0 & +56.4 & 161.7 & N & \\
\hline $27,4,135-137$ & 250.16 & .14 & +72.3 & 51.4 & +73.6 & 154.2 & $\mathrm{~N}$ & 8.2 \\
\hline
\end{tabular}




\begin{tabular}{|c|c|c|c|c|c|c|c|c|}
\hline $\begin{array}{l}\text { Core, Section, } \\
\text { Interval }(\mathrm{cm})\end{array}$ & $\begin{array}{l}\text { Depth } \\
\text { (mbsf) }\end{array}$ & $\begin{array}{c}\mathrm{J} \\
\mathrm{NRM} \\
(\mathrm{mA} / \mathrm{m})\end{array}$ & $\begin{array}{l}\text { Incl. } \\
\text { NRM } \\
\text { (deg) }\end{array}$ & $\begin{array}{l}\text { Decl. } \\
\text { NRM } \\
\text { (deg) }\end{array}$ & $\begin{array}{c}\text { Incl } \\
\text { stable } \\
\text { (deg) }\end{array}$ & $\begin{array}{c}\text { Decl. } \\
\text { stable } \\
\text { (deg) }\end{array}$ & Pol. & $\begin{array}{l}\mathrm{MDF} \\
(\mathrm{mT}) \\
\end{array}$ \\
\hline $27,5,35-37$ & 250.66 & .07 & +34.6 & 120.1 & +52.4 & 167.1 & $\mathrm{~N}$ & 4.1 \\
\hline $27,5,72-74$ & 251.03 & .08 & -61.9 & 77.9 & -68.9 & 64.3 & $\mathrm{R}$ & 14.4 \\
\hline $27,5,125-127$ & 251.56 & .12 & -56.3 & 73.2 & -64.6 & 64.3 & $\mathrm{R}$ & 18.7 \\
\hline $27,6,34-36$ & 252.15 & .07 & +45.2 & 123.3 & -68.4 & 64.4 & $\mathrm{R}$ & 4.7 \\
\hline $27,6,73-75$ & 252.54 & .12 & -50.7 & 79.2 & -55.9 & 89.2 & $\mathrm{R}$ & 4.6 \\
\hline $27,6,125-127$ & 253.06 & .07 & +75.8 & 134.8 & -47.6 & 63.1 & $\mathrm{R}$ & 14.6 \\
\hline $28,1,35-37$ & 254.46 & .08 & +29.4 & 351.6 & -12.5 & 336.8 & $\mathrm{R}$ & 4.6 \\
\hline $28,1,85-87$ & 254.96 & .15 & -14.6 & 349.5 & -51.4 & 46.9 & $\mathrm{R}$ & 47.5 \\
\hline $28,1,135-137$ & 255.46 & .06 & +43.6 & 32.2 & -15.2 & 54.6 & $\mathrm{R}$ & \\
\hline $28,2,35-37$ & 255.96 & .08 & +50.0 & 31.5 & +49.0 & 28.5 & $\mathrm{~N}$ & 27.9 \\
\hline $28,2,85-87$ & 256.46 & .05 & +14.8 & 95.2 & +52.5 & 96.8 & $\mathrm{~N}$ & \\
\hline $28,2,135-137$ & 256.96 & .10 & +77.8 & 100.0 & -63.2 & 127.0 & & 7.7 \\
\hline $28,3,35-37$ & 257.46 & .08 & +62.6 & 84.1 & +67.0 & 352.3 & $\mathrm{~N}$ & 13.5 \\
\hline $28,3,85-87$ & 257.96 & .06 & +50.0 & 283.4 & +66.1 & 276.1 & $\mathrm{~N}$ & 7.5 \\
\hline $28,3,135-137$ & 258.46 & .10 & +81.9 & 352.0 & +64.9 & 310.1 & N & 20.8 \\
\hline $28,4,35-37$ & 258.96 & .05 & +76.1 & 18.1 & +80.5 & 299.8 & $\mathrm{~N}$ & 7.0 \\
\hline $28,4,85-87$ & 259.46 & .03 & +73.0 & 313.9 & +69.5 & 306.6 & $\mathrm{~N}$ & 9.1 \\
\hline $28,4,135-137$ & 259.96 & .05 & +62.2 & 110.0 & +68.6 & 320.6 & $\mathrm{~N}$ & 8.8 \\
\hline $28,5,35-37$ & 260.46 & .07 & +49.0 & 39.1 & +56.7 & 331.5 & $\mathrm{~N}$ & 26.1 \\
\hline $28,5,85-87$ & 260.96 & .06 & +79.6 & 66.5 & +82.1 & 329.7 & $\mathrm{~N}$ & 26.4 \\
\hline $28,6,35-37$ & 261.96 & .08 & +40.5 & 292.0 & +61.0 & 336.1 & $\mathrm{~N}$ & 14.4 \\
\hline $28,6,85-87$ & 262.46 & .05 & +57.7 & 300.9 & +68.1 & 306.5 & $\mathrm{~N}$ & 14.9 \\
\hline $28,6,135-137$ & 262.96 & .05 & +70.7 & 278.8 & +70.7 & 315.5 & $\mathrm{~N}$ & 13.7 \\
\hline $29,1,35-37$ & 264.26 & .05 & +49.3 & 97.0 & +51.2 & 82.5 & $\mathrm{~N}$ & \\
\hline $29,1,85-87$ & 264.76 & .05 & +60.6 & 62.7 & +66.3 & 66.2 & $\mathrm{~N}$ & \\
\hline $29,1,135-137$ & 265.26 & .08 & +76.0 & 328.2 & +75.5 & 70.9 & $\mathrm{~N}$ & 4.5 \\
\hline $29,2,35-37$ & 265.76 & .06 & +58.8 & 53.4 & +70.3 & 52.6 & $\mathrm{~N}$ & 19.5 \\
\hline $29,2,85-87$ & 266.26 & .11 & +67.8 & 24.9 & +76.1 & 58.3 & N & 8.7 \\
\hline $29,2,135-137$ & 266.76 & .17 & +39.8 & 65.9 & +61.0 & 69.6 & $\mathrm{~N}$ & 4.0 \\
\hline $29,3,35-37$ & 267.26 & .14 & +47.6 & 52.2 & +67.3 & 47.7 & $\mathrm{~N}$ & 14.9 \\
\hline $29,3,85-87$ & 267.76 & .09 & +82.0 & 196.2 & +69.0 & 79.8 & $\mathrm{~N}$ & 19.8 \\
\hline $29,3,135-137$ & 268.26 & .04 & +63.5 & 15.4 & +69.5 & 46.4 & $\mathrm{~N}$ & \\
\hline $29,4,35-37$ & 268.76 & .11 & +22.9 & 329.4 & +64.5 & 40.2 & $\mathrm{~N}$ & 21.6 \\
\hline $29,4,85-87$ & 269.26 & .07 & +58.9 & 22.0 & +70.0 & 45.0 & $\mathrm{~N}$ & 11.5 \\
\hline $29,4,135-137$ & 269.76 & .08 & +17.5 & 294.8 & +67.2 & 66.8 & $\mathrm{~N}$ & 19.6 \\
\hline $29,5,35-37$ & 270.26 & .04 & +53.0 & 239.7 & +71.2 & 68.4 & $\mathrm{~N}$ & \\
\hline $29,5,85-87$ & 270.76 & .19 & +80.8 & 63.0 & +75.9 & 69.7 & $\mathrm{~N}$ & 11.0 \\
\hline $29,6,35-37$ & 271.76 & .09 & +70.0 & 51.0 & +67.0 & 49.1 & $\mathrm{~N}$ & 3.6 \\
\hline $29,6,85-87$ & 272.26 & .08 & +55.9 & 35.1 & +72.5 & 48.4 & $\mathrm{~N}$ & 4.0 \\
\hline $29,6,135-137$ & 272.76 & .10 & +71.9 & 36.4 & +74.6 & 57.3 & $\mathrm{~N}$ & 13.2 \\
\hline $31,1,85-87$ & 284.36 & .48 & -26.2 & 121.3 & +85.5 & 130.6 & $\mathrm{~N}$ & 3.2 \\
\hline $31,1,135-137$ & 284.86 & .37 & +80.7 & 141.4 & +75.1 & 127.0 & $\mathrm{~N}$ & 4.5 \\
\hline $31,2,35-37$ & 285.36 & .25 & +76.8 & 76.8 & +86.4 & 100.7 & $\mathrm{~N}$ & 6.8 \\
\hline $31,2,85-87$ & 285.86 & .22 & +74.8 & 310.4 & +75.9 & 126.9 & $\mathrm{~N}$ & 7.9 \\
\hline $31,2,135-137$ & 286.36 & .27 & +86.3 & 327.9 & +77.3 & 135.1 & $\mathrm{~N}$ & 9.2 \\
\hline $31,3,35-37$ & 286.86 & .17 & +88.1 & 125.1 & +71.0 & 137.1 & $\mathrm{~N}$ & 5.6 \\
\hline $31,3,85-87$ & 287.36 & .17 & +71.3 & 148.3 & +71.7 & 133.3 & $\mathrm{~N}$ & 10.9 \\
\hline
\end{tabular}


Table A4 (continued).

\begin{tabular}{|c|c|c|c|c|c|c|c|c|}
\hline $\begin{array}{l}\text { Core, Section, } \\
\text { Interval }(\mathrm{cm})\end{array}$ & $\begin{array}{l}\text { Depth } \\
\text { (mbsf) }\end{array}$ & $\begin{array}{c}\mathrm{J} \\
\mathrm{NRM} \\
(\mathrm{mA} / \mathrm{m})\end{array}$ & $\begin{array}{l}\text { Incl. } \\
\text { NRM } \\
\text { (deg) }\end{array}$ & $\begin{array}{l}\text { Decl. } \\
\text { NRM } \\
\text { (deg) }\end{array}$ & $\begin{array}{c}\text { Incl } \\
\text { stable } \\
\text { (deg) }\end{array}$ & $\begin{array}{l}\text { Decl. } \\
\text { stable } \\
\text { (deg) }\end{array}$ & Pol. & $\begin{array}{l}\mathrm{MDF} \\
(\mathrm{mT})\end{array}$ \\
\hline $31,3,135-137$ & 287.86 & .39 & +75.5 & 343.0 & +68.3 & 131.5 & $\mathrm{~N}$ & 12.9 \\
\hline $31,4,35-37$ & 288.36 & .31 & +45.5 & 63.4 & +67.5 & 127.4 & $\mathrm{~N}$ & 5.5 \\
\hline $31,4,85-87$ & 288.86 & .24 & +82.8 & 131.7 & +76.9 & 118.9 & $\mathrm{~N}$ & 20.0 \\
\hline $31,4,135-137$ & 289.36 & .22 & +75.1 & 141.6 & +76.3 & 138.5 & $\mathrm{~N}$ & 13.6 \\
\hline $31,5,28-30$ & 289.79 & .39 & +26.3 & 124.5 & +60.1 & 141.0 & $\mathrm{~N}$ & 3.0 \\
\hline $31,5,130-132$ & 290.81 & .13 & +74.6 & 122.8 & +74.7 & 136.2 & $\mathrm{~N}$ & 14.3 \\
\hline $31,6,35-37$ & 291.36 & .12 & +62.3 & 105.4 & +65.8 & 139.4 & $\mathrm{~N}$ & 9.5 \\
\hline $31,6,135-137$ & 292.36 & .13 & +73.1 & 97.6 & +64.9 & 118.3 & $\mathrm{~N}$ & 9.4 \\
\hline $32,1,35-37$ & 293.66 & .24 & +81.0 & 358.5 & +75.5 & 133.0 & $\mathrm{~N}$ & 3.5 \\
\hline $32,1,85-87$ & 294.16 & .15 & +76.2 & 71.9 & +82.0 & 131.5 & $\mathrm{~N}$ & 4.6 \\
\hline $32,1,135-137$ & 294.66 & .23 & +84.1 & 167.0 & +68.3 & 122.4 & $\mathrm{~N}$ & 4.1 \\
\hline $32,2,35-37$ & 295.16 & .11 & +75.7 & 305.2 & +61.1 & 127.2 & $\mathrm{~N}$ & 7.4 \\
\hline $32,2,85-87$ & 295.66 & .20 & +85.5 & 84.7 & +85.1 & 118.9 & $\mathrm{~N}$ & 4.4 \\
\hline $32,3,38-40$ & 296.69 & .31 & -84.7 & 334.1 & -85.7 & 302.8 & $\mathrm{R}$ & 3.2 \\
\hline $33,1,85-87$ & 303.96 & .10 & +76.0 & 306.5 & +72.2 & 352.2 & $\mathrm{~N}$ & 9.8 \\
\hline $33,1,135-137$ & 304.46 & .03 & +10.7 & 223.6 & -76.2 & 221.5 & $\mathrm{R}$ & \\
\hline $33,2,35-37$ & 304.96 & .06 & +74.1 & 101.4 & +74.4 & 82.1 & $\mathrm{~N}$ & 12.1 \\
\hline $34,1,44-46$ & 313.35 & .11 & +68.4 & 53.2 & +70.7 & 38.1 & $\mathrm{~N}$ & 8.2 \\
\hline $34,1,120-122$ & 314.11 & .05 & +70.0 & 358.3 & +69.5 & 33.8 & $\mathrm{~N}$ & 5.8 \\
\hline $34,2,44-46$ & 314.85 & .10 & +67.4 & 66.3 & +67.5 & 45.9 & $\mathrm{~N}$ & 9.5 \\
\hline $34,2,120-122$ & 315.61 & .06 & +61.1 & 351.6 & +59.5 & 15.9 & $\mathrm{~N}$ & 15.4 \\
\hline $34,3,44-46$ & 316.35 & .03 & +72.5 & 109.8 & +69.1 & 28.4 & $\mathrm{~N}$ & \\
\hline $35,1,54-56$ & 323.25 & .05 & +82.8 & 213.9 & +76.0 & 95.7 & $\mathrm{~N}$ & \\
\hline $41,1,28-30$ & 381.39 & .33 & +60.7 & 97.7 & +81.9 & 351.9 & $\mathrm{~N}$ & 7.3 \\
\hline $41,1,78-80$ & 381.89 & .21 & +66.7 & 46.0 & +71.7 & 3.2 & $\mathrm{~N}$ & 16.1 \\
\hline $41,1,129-131$ & 382.40 & .26 & +74.6 & 46.3 & +67.5 & 22.2 & $\mathrm{~N}$ & 3.8 \\
\hline $42,3,28-30$ & 393.99 & .04 & +50.5 & 109.4 & -63.3 & 121.0 & R & \\
\hline $42,3,78-80$ & 394.49 & .20 & -25.9 & 183.2 & -68.8 & 143.2 & $\mathrm{R}$ & \\
\hline $42,3,129-131$ & 395.00 & .46 & +17.6 & 99.1 & -64.1 & 134.8 & $\mathrm{R}$ & 3.0 \\
\hline
\end{tabular}

$\mathrm{J}_{\mathrm{NRM}}$, Decl.NRM, Incl.NRM - intensity, inclination, declination of the natural remanent magnetization; Incl.stable, Decl.stable inclination, declination of the stable remanence after AF demagnetization; Pol., N, R - polarity, normal, reversed; MDF - median destructive field. 
Table A5. Paleomagnetic properties of samples from Hole 644A.

\begin{tabular}{|c|c|c|c|c|c|c|c|c|}
\hline $\begin{array}{l}\text { Core, Section, } \\
\text { Interval }(\mathrm{cm})\end{array}$ & $\begin{array}{l}\text { Depth } \\
\text { (mbsf) }\end{array}$ & $\begin{array}{c}\mathrm{J} \\
\mathrm{NRM} \\
(\mathrm{mA} / \mathrm{m})\end{array}$ & $\begin{array}{l}\text { Incl. } \\
\text { NRM } \\
\text { (deg) }\end{array}$ & $\begin{array}{l}\text { Decl. } \\
\text { NRM } \\
\text { (deg) }\end{array}$ & $\begin{array}{l}\text { Incl } \\
\text { stable } \\
\text { (deg) }\end{array}$ & $\begin{array}{l}\text { Decl. } \\
\text { stable } \\
\text { (deg) }\end{array}$ & Pol. & $\begin{array}{l}\text { MDF } \\
(\mathrm{mT})\end{array}$ \\
\hline $1,1,35-37$ & .36 & 30.83 & +65.7 & 155.2 & +68.1 & 151.7 & $\mathrm{~N}$ & 27.3 \\
\hline $1,1,85-87$ & .86 & 12.87 & +75.8 & 116.8 & +72.6 & 117.9 & $\mathrm{~N}$ & 20.1 \\
\hline $1,1,135-137$ & 1.36 & 9.09 & +75.9 & 149.2 & +75.0 & 149.5 & $\mathrm{~N}$ & 24.3 \\
\hline $1,2,35-37$ & 1.86 & 5.95 & +53.4 & 158.4 & +69.8 & 158.5 & $\mathrm{~N}$ & 31.1 \\
\hline $1,2,85-87$ & 2.36 & 6.25 & +44.0 & 128.9 & +67.2 & 124.4 & $\mathrm{~N}$ & 28.8 \\
\hline $1,2,135-137$ & 2.86 & 14.10 & +43.0 & 156.6 & +64.0 & 135.2 & $\mathrm{~N}$ & 27.3 \\
\hline $1,3,35-37$ & 3.36 & 4.52 & +6.1 & 189.5 & +56.5 & 176.7 & $\mathrm{~N}$ & 24.4 \\
\hline $1,3,85-87$ & 3.86 & 1.80 & +32.3 & 182.2 & +63.4 & 149.5 & $\mathrm{~N}$ & 35.2 \\
\hline $1,3,135-137$ & 4.36 & 13.23 & +59.3 & 147.1 & +71.6 & 136.1 & $\mathrm{~N}$ & \\
\hline $1,4,35-37$ & 4.86 & 8.79 & +64.9 & 130.5 & +66.4 & 140.4 & $\mathrm{~N}$ & 25.6 \\
\hline $1,4,85-87$ & 5.36 & 3.32 & -26.1 & 172.4 & +67.6 & 156.8 & $\mathrm{~N}$ & 29.6 \\
\hline $1,4,135-137$ & 5.86 & 4.87 & -38.6 & 23.2 & +77.5 & 147.3 & $\mathrm{~N}$ & 34.9 \\
\hline $1,5,35-37$ & 6.36 & 15.91 & +69.0 & 155.7 & +69.0 & 153.3 & $\mathrm{~N}$ & 25.1 \\
\hline $1,5,85-87$ & 6.86 & 17.90 & +67.4 & 135.1 & +66.9 & 132.9 & $\mathrm{~N}$ & 20.4 \\
\hline $1,5,135-137$ & 7.36 & 25.97 & +80.4 & 159.2 & +82.8 & 159.4 & $\mathrm{~N}$ & 38.9 \\
\hline $1,6,35-37$ & 7.86 & 20.59 & +75.3 & 117.3 & +76.6 & 112.5 & $\mathrm{~N}$ & 25.6 \\
\hline $1,6,85-87$ & 8.36 & 31.45 & +60.5 & 88.7 & +72.8 & 109.8 & $\mathrm{~N}$ & 16.0 \\
\hline $1,6,135-137$ & 8.86 & 19.19 & +72.3 & 132.2 & +75.1 & 128.3 & $\mathrm{~N}$ & 31.0 \\
\hline $2,1,35-37$ & 9.56 & 9.56 & +47.2 & 247.4 & +23.2 & 344.5 & & 24.5 \\
\hline $2,1,85-87$ & 10.06 & 3.26 & +27.8 & 87.8 & -13.8 & 37.8 & $\mathrm{R}$ & 38.6 \\
\hline $2,1,135-137$ & 10.56 & 11.63 & +55.7 & 205.5 & +67.2 & 219.3 & $\mathrm{~N}$ & 27.9 \\
\hline $2,2,35-37$ & 11.06 & 42.01 & +70.7 & 225.3 & +71.4 & 222.0 & $\mathrm{~N}$ & 32.3 \\
\hline $2,2,85-87$ & 11.56 & 5.45 & +69.5 & 167.4 & +84.0 & 223.1 & $\mathrm{~N}$ & 33.9 \\
\hline $2,2,135-137$ & 12.06 & 12.83 & +74.3 & 200.1 & +74.4 & 225.0 & $\mathrm{~N}$ & 29.5 \\
\hline $2,3,35-37$ & 12.56 & 8.93 & +72.7 & 152.1 & +72.3 & 174.8 & $\mathrm{~N}$ & 9.9 \\
\hline $2,3,85-87$ & 13.06 & 26.39 & +65.6 & 171.9 & +63.7 & 172.6 & $\mathrm{~N}$ & 25.7 \\
\hline $2,3,135-137$ & 13.56 & 6.29 & +51.6 & 233.2 & +69.0 & 191.3 & $\mathrm{~N}$ & 23.2 \\
\hline $2,4,35-37$ & 14.06 & 53.45 & +73.0 & 166.1 & +76.2 & 163.9 & $\mathrm{~N}$ & 34.7 \\
\hline $2,4,85-87$ & 14.56 & 75.32 & +50.8 & 237.8 & +63.3 & 174.5 & $\mathrm{~N}$ & 15.8 \\
\hline $2,4,93-95$ & 14.64 & 1.59 & +73.8 & 109.2 & -3.0 & 310.2 & $\mathrm{R}$ & 16.4 \\
\hline $2,4,114-116$ & 14.85 & 67.84 & +68.9 & 130.5 & +6.3 & 237.5 & & 29.5 \\
\hline $2,4,128-130$ & 14.99 & 101.15 & +73.8 & 140.8 & +8.7 & 232.4 & & 30.0 \\
\hline $2,4,135-137$ & 15.06 & 33.28 & +68.9 & 157.7 & -65.7 & 334.7 & $\mathrm{R}$ & 31.6 \\
\hline $2,4,137-139$ & 15.08 & 21.41 & +69.7 & 134.7 & -12.7 & 249.0 & $\mathrm{R}$ & 28.2 \\
\hline $2,4,146-148$ & 15.17 & 82.12 & +70.1 & 129.7 & +71.6 & 146.6 & $\mathrm{~N}$ & 28.6 \\
\hline $2,5, \quad 5-7$ & 15.26 & 52.67 & +74.8 & 148.1 & +75.8 & 161.8 & $\mathrm{~N}$ & 29.1 \\
\hline $2,5,15-17$ & 15.36 & 63.78 & +76.4 & 143.7 & +76.6 & 159.1 & $\mathrm{~N}$ & 30.1 \\
\hline $2,5,29-31$ & 15.50 & 43.23 & +67.9 & 124.7 & +69.4 & 136.1 & $\mathrm{~N}$ & 29.9 \\
\hline $2,5,35-37$ & 15.56 & 19.68 & +68.0 & 126.4 & +68.5 & 132.5 & $\mathrm{~N}$ & 29.7 \\
\hline $3,1,35-37$ & 16.56 & 42.27 & +64.6 & 141.0 & +67.8 & 143.0 & $\mathrm{~N}$ & 30.5 \\
\hline $3,1,85-87$ & 17.06 & 17.29 & +68.2 & 117.3 & +70.0 & 112.6 & $\mathrm{~N}$ & 25.8 \\
\hline $3,1,135-137$ & 17.56 & 12.23 & +71.3 & 110.1 & +68.7 & 124.0 & $\mathrm{~N}$ & 21.9 \\
\hline $3,2,35-37$ & 18.06 & 5.83 & +72.6 & 102.9 & +72.7 & 126.9 & $\mathrm{~N}$ & 25.1 \\
\hline $3,2,85-87$ & 18.56 & 8.03 & +54.0 & 169.5 & +65.9 & 136.4 & $\mathrm{~N}$ & 18.4 \\
\hline $3,2,135-137$ & 19.06 & 13.53 & +74.3 & 192.5 & +76.4 & 127.4 & $\mathrm{~N}$ & 17.1 \\
\hline $3,3,33-35$ & 19.54 & 35.15 & +67.2 & 132.9 & +69.3 & 130.6 & $\mathrm{~N}$ & 29.8 \\
\hline $3,3,85-37$ & 20.06 & 39.90 & +73.6 & 155.2 & +77.8 & 145.1 & $\mathrm{~N}$ & 28.1 \\
\hline
\end{tabular}


Table A5 (continued).

\begin{tabular}{|c|c|c|c|c|c|c|c|c|}
\hline $\begin{array}{l}\text { Core, Section, } \\
\text { Interval }(\mathrm{cm})\end{array}$ & $\begin{array}{l}\text { Depth } \\
\text { (mbsf) }\end{array}$ & $\begin{array}{c}\mathrm{J} \\
\mathrm{NRM} \\
(\mathrm{mA} / \mathrm{m})\end{array}$ & $\begin{array}{l}\text { Incl. } \\
\text { NRM } \\
\text { (deg) }\end{array}$ & $\begin{array}{l}\text { Decl. } \\
\text { NRM } \\
\text { (deg) }\end{array}$ & $\begin{array}{c}\text { Incl } \\
\text { stable } \\
\text { (deg) }\end{array}$ & $\begin{array}{l}\text { Decl. } \\
\text { stable } \\
\text { (deg) }\end{array}$ & Pol. & $\begin{array}{l}\text { MDF } \\
(\mathrm{mT})\end{array}$ \\
\hline $3,4,35-37$ & 21.06 & 60.29 & +65.2 & 168.1 & +69.7 & 167.3 & $\mathrm{~N}$ & 30.0 \\
\hline $3,4,85-87$ & 21.56 & 19.92 & +75.8 & 162.1 & +76.8 & 171.8 & $\mathrm{~N}$ & 26.8 \\
\hline $3,4,135-137$ & 22.06 & 11.78 & +71.7 & 169.0 & +69.1 & 167.9 & $\mathrm{~N}$ & 19.1 \\
\hline $3,5,35-37$ & 22.56 & 45.51 & +72.9 & 165.8 & +73.5 & 172.2 & $\mathrm{~N}$ & 26.4 \\
\hline $3,5,38-40$ & 22.59 & 61.43 & +77.3 & 150.4 & +79.8 & 171.1 & $\mathrm{~N}$ & 25.9 \\
\hline $3,5,54-56$ & 22.75 & 37.93 & +76.9 & 138.7 & +76.4 & 162.5 & $\mathrm{~N}$ & 26.8 \\
\hline $3,5,71-73$ & 22.92 & 10.33 & +35.3 & 132.9 & -43.5 & 82.8 & $\mathrm{R}$ & 12.2 \\
\hline $3,5,85-87$ & 23.06 & 10.32 & +14.2 & 203.5 & -45.8 & 148.8 & $\mathrm{R}$ & 4.6 \\
\hline $3,5,89-91$ & 23.10 & 10.55 & +49.6 & 117.4 & -23.8 & 142.2 & $\mathrm{R}$ & 15.9 \\
\hline $3,5,97-99$ & 23.18 & 40.18 & +76.4 & 148.1 & +77.4 & 163.5 & $\mathrm{~N}$ & 26.3 \\
\hline $3,5,109-111$ & 23.30 & 54.77 & +69.8 & 146.0 & +71.4 & 158.4 & $\mathrm{~N}$ & 28.9 \\
\hline $3,5,117-119$ & 23.38 & 55.35 & +74.4 & 153.6 & +7 & 163.5 & $\mathrm{~N}$ & 27.1 \\
\hline $3,5,127-129$ & 23.48 & 55.13 & +77.8 & 152.9 & +78.3 & 172.0 & $\mathrm{~N}$ & 27.1 \\
\hline $3,5,135-137$ & 23.56 & 33.75 & +70.9 & 156.4 & +70.7 & 152.0 & $\mathrm{~N}$ & 25.9 \\
\hline $3,6,35-37$ & 24.06 & 13.39 & +77.4 & 177.9 & +82.2 & 186.4 & $\mathrm{~N}$ & 24.4 \\
\hline $4,1,18-20$ & 25.89 & 20.49 & +64.0 & 122.5 & +66.5 & 108.8 & $\mathrm{~N}$ & 23.8 \\
\hline $4,1,85-87$ & 26.56 & 23.97 & +84.7 & 25.0 & +79.8 & 356.6 & $\mathrm{~N}$ & 25.0 \\
\hline $4,1,135-137$ & 27.06 & 47.29 & +80.9 & 33.7 & +80.0 & 34.1 & $\mathrm{~N}$ & 24.3 \\
\hline $4,1,148-150$ & 27.19 & 18.27 & +77.8 & 67.7 & +82.4 & 47.0 & $\mathrm{~N}$ & 26.4 \\
\hline $4,2, \quad 5-7$ & 27.26 & 14.52 & +79.0 & 71.7 & +56.5 & 102.5 & $\mathrm{~N}$ & 7.2 \\
\hline $4,2,16-18$ & 27.37 & 6.26 & +65.1 & 97.3 & -72.4 & 121.2 & $\mathrm{R}$ & 9.2 \\
\hline $4,2,30-32$ & 27.51 & 4.98 & +65.0 & 32.2 & -77.6 & 100.4 & $\mathrm{R}$ & 6.7 \\
\hline $4,2,35-37$ & 27.56 & 1.69 & -67.3 & 111.9 & -81.1 & 118.4 & $\mathrm{R}$ & \\
\hline $4,2,48-50$ & 27.69 & 16.56 & +77.4 & 32.1 & +79.6 & 27.2 & $\mathrm{~N}$ & 20.3 \\
\hline $4,2,55-57$ & 27.76 & 19.85 & +83.3 & 77.9 & +83.3 & 9.8 & $\mathrm{~N}$ & 20.6 \\
\hline $4,2,64-66$ & 27.85 & 20.24 & +57.8 & 27.7 & +79.2 & 348.9 & $\mathrm{~N}$ & 13.6 \\
\hline $4,2,79-81$ & 28.00 & 6.02 & +46.9 & 13.5 & +81.0 & 338.7 & $\mathrm{~N}$ & \\
\hline $4,2,85-87$ & 28.06 & 26.79 & +85.0 & 57.0 & +86.4 & 3.3 & $\mathrm{~N}$ & 21.0 \\
\hline $4,2,135-137$ & 28.56 & 61.31 & +75.0 & 325.9 & +73.1 & 326.4 & $\mathrm{~N}$ & 28.5 \\
\hline $4,3,35-37$ & 29.06 & 58. & +86.3 & 340.4 & +82.7 & 344.9 & $\mathrm{~N}$ & 22.4 \\
\hline $4,3,80-82$ & 29.51 & 36. & +82.4 & 357.4 & +78.7 & 347.7 & $\mathrm{~N}$ & 26.8 \\
\hline $4,3,135-137$ & 30.06 & 7 & $+\varepsilon$ & 51 & +8 & 5.2 & $\mathrm{~N}$ & 13.7 \\
\hline $4,4,35-37$ & 30.56 & 20.08 & $+\varepsilon$ & 21.0 & $+\varepsilon$ & 16.5 & $\mathrm{~N}$ & 27.7 \\
\hline $4,4,85-87$ & 31.06 & 5.63 & +79.6 & 3.5 & +76.6 & 353.8 & $\mathrm{~N}$ & 31.2 \\
\hline $4,4,135-137$ & 31.56 & 32.53 & +76.8 & 308.1 & +76.8 & 302.0 & $\mathrm{~N}$ & 34.3 \\
\hline $4,5,35-37$ & 32.06 & 39.42 & +73.9 & 311.4 & +78.6 & 291.7 & $\mathrm{~N}$ & 35.6 \\
\hline $4,5,85-87$ & 32.56 & 4.20 & +69.2 & 305.7 & +83.8 & 329.8 & N & 15.2 \\
\hline $4,5,135-137$ & 33.06 & 10.09 & +87.2 & 219.4 & +87.4 & 320.1 & $\mathrm{~N}$ & 24.7 \\
\hline $4,6,35-37$ & 33.56 & 43.55 & +78.5 & 335.8 & +75.1 & 332.6 & $\mathrm{~N}$ & 25.1 \\
\hline $4,6,85-87$ & 34.06 & 46.99 & +80.6 & 20.8 & +80.3 & 6.5 & $\mathrm{~N}$ & 26.7 \\
\hline $5,1,34-36$ & 35.55 & 198.38 & +78.5 & 68.6 & +80.3 & 355.3 & $\mathrm{~N}$ & 39.9 \\
\hline $5,1,84-86$ & 36.05 & 24.60 & +84.7 & 311.6 & +80.9 & 336.0 & $\mathrm{~N}$ & 22.5 \\
\hline $5,1,134-136$ & 36.55 & 2.71 & +74.2 & 274.7 & +74.6 & 293.5 & $\mathrm{~N}$ & 13.7 \\
\hline $5,2, \quad 4-6$ & 36.75 & .73 & +80.7 & 345.7 & -57.0 & 153.3 & $\mathrm{R}$ & 9.8 \\
\hline $5,2,14-16$ & 36.85 & .72 & +64.2 & 39.5 & +79.4 & 331.8 & $\mathrm{~N}$ & 18.0 \\
\hline $5,2,30-32$ & 37.01 & .44 & +76.2 & 316.4 & +14.0 & 292.1 & & 13.4 \\
\hline $5,2,35-37$ & 37.06 & 1.27 & +78.5 & 288.2 & -55.8 & 231.9 & $\mathrm{R}$ & 4.6 \\
\hline $5,2,46-48$ & 37.17 & 1.30 & +62.7 & 85.5 & -63.5 & 163.6 & $\mathrm{R}$ & 5.0 \\
\hline
\end{tabular}


Table A5 (continued).

\begin{tabular}{|c|c|c|c|c|c|c|c|c|}
\hline $\begin{array}{l}\text { Core, Section, } \\
\text { Interval }(\mathrm{cm})\end{array}$ & $\begin{array}{l}\text { Depth } \\
\text { (mbsf) }\end{array}$ & 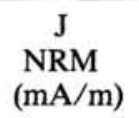 & $\begin{array}{l}\text { Incl. } \\
\text { NRM } \\
\text { (deg) }\end{array}$ & $\begin{array}{l}\text { Decl. } \\
\text { NRM } \\
\text { (deg) }\end{array}$ & $\begin{array}{l}\text { Incl } \\
\text { stable } \\
\text { (deg) }\end{array}$ & $\begin{array}{l}\text { Decl. } \\
\text { stable } \\
\text { (deg) }\end{array}$ & Pol. & $\begin{array}{l}\text { MDF } \\
(\mathrm{mT})\end{array}$ \\
\hline $5,2,55-57$ & 37.26 & 3.36 & +80.5 & 70.6 & +77.1 & 329.6 & $\mathrm{~N}$ & 20.9 \\
\hline $5,2,73-75$ & 37.44 & 9.71 & +83.7 & 294.8 & +82.0 & 295.2 & $\mathrm{~N}$ & 27.5 \\
\hline $5,2,85-87$ & 37.56 & 39.97 & +79.5 & 290.3 & +78.5 & 297.4 & $\mathrm{~N}$ & 41.2 \\
\hline $5,2,128-130$ & 37.99 & 37.55 & +76.2 & 330.8 & +76.3 & 328.5 & $\mathrm{~N}$ & 31.7 \\
\hline $5,3,35-37$ & 38.56 & 30.35 & +75.0 & 305.0 & +75.0 & 312.5 & $\mathrm{~N}$ & 40.2 \\
\hline $5,3,85-87$ & 39.06 & 57.82 & +84.0 & 345.7 & +83.4 & 344.8 & $\mathrm{~N}$ & 45.4 \\
\hline $5,3,128-130$ & 39.49 & 32.82 & +83.0 & 341.7 & +81.8 & 329.6 & $\mathrm{~N}$ & 34.9 \\
\hline $5,4,35-37$ & 40.06 & 9.48 & +81.4 & 276.8 & +78.2 & 300.9 & $\mathrm{~N}$ & 41.8 \\
\hline $5,4,85-87$ & 40.56 & 22.39 & +78.2 & 18.7 & +76.4 & 12.1 & $\mathrm{~N}$ & 26.8 \\
\hline $5,4,129-131$ & 41.00 & 16.75 & +77.6 & 25.5 & +76.4 & 14.4 & $\mathrm{~N}$ & 32.1 \\
\hline $5,5,35-37$ & 41.56 & 53.06 & +84.8 & 311.2 & +83.3 & 307.3 & $\mathrm{~N}$ & 39.2 \\
\hline $5,5,85-87$ & 42.06 & 10.83 & +82.8 & 333.9 & +76.9 & 315.4 & $\mathrm{~N}$ & 40.5 \\
\hline $5,5,135-137$ & 42.56 & 3.25 & +67.3 & 122.6 & +78.1 & 312.8 & $\mathrm{~N}$ & 5.3 \\
\hline $6,1,35-37$ & 45.06 & 27.45 & +80.6 & 163.3 & +82.4 & 34.8 & $N$ & 32.9 \\
\hline $6,1,85-87$ & 45.56 & 2.27 & +40.9 & 119.6 & +71.9 & 59.3 & $\mathrm{~N}$ & 20.5 \\
\hline $6,1,135-137$ & 46.06 & 35.35 & +76.0 & 98.4 & +76.4 & 93.3 & $\mathrm{~N}$ & 48.7 \\
\hline $6,2,35-37$ & 46.56 & 3.74 & +57.3 & 112.8 & +67.7 & 101.6 & $\mathrm{~N}$ & 12.5 \\
\hline $6,2,85-87$ & 47.06 & 7.78 & +71.5 & 106.5 & +70.0 & 107.6 & $\mathrm{~N}$ & 22.6 \\
\hline $6,2,135-137$ & 47.56 & 7.35 & +73.9 & 111.7 & +77.0 & 99.2 & $\mathrm{~N}$ & 25.8 \\
\hline $6,3,35-37$ & 48.06 & 31.73 & +83.2 & 103.6 & +83.8 & 98.6 & $\mathrm{~N}$ & 25.1 \\
\hline $6,3,85-87$ & 48.56 & 61.58 & +74.3 & 90.0 & +73.9 & 89.5 & $\mathrm{~N}$ & 25.0 \\
\hline $6,3,135-137$ & 49.06 & 22.83 & +69.2 & 113.0 & +72.3 & 99.4 & $\mathrm{~N}$ & 22.0 \\
\hline $6,4,35-37$ & 49.56 & 73.18 & +85.2 & 99.2 & +85.8 & 92.8 & $\mathrm{~N}$ & 26.2 \\
\hline $6,4,85-87$ & 50.06 & 30.51 & +83.2 & 101.2 & +84.4 & 103.2 & $\mathrm{~N}$ & 31.4 \\
\hline $6,4,105-107$ & 50.26 & 8.61 & +83.1 & 130.1 & +81.9 & 90.3 & $\mathrm{~N}$ & 20.7 \\
\hline $6,5,35-37$ & 51.06 & 46.36 & +87.8 & 153.8 & +83.5 & 106.0 & $\mathrm{~N}$ & 23.0 \\
\hline $6,5,85-87$ & 51.56 & 46.55 & +77.7 & 86.3 & +76.5 & 86.2 & $\mathrm{~N}$ & 23.0 \\
\hline $6,5,135-137$ & 52.06 & 24.84 & +82.2 & 123.8 & +84.3 & 106.0 & $\mathrm{~N}$ & 20.8 \\
\hline $6,6,35-37$ & 52.56 & 3.07 & +85.3 & 73.9 & +83.9 & 104.4 & $\mathrm{~N}$ & 16.5 \\
\hline $6,6,85-87$ & 53.06 & 26.68 & +69.8 & 104.0 & +73.8 & 100.5 & $\mathrm{~N}$ & 13.6 \\
\hline $7,1,18-20$ & 53.09 & 3.20 & +71.5 & 149.3 & +67.3 & 165.6 & $\mathrm{~N}$ & 21.7 \\
\hline $7,1,71-73$ & 53.62 & 35.73 & +82.5 & 235.4 & +83.2 & 206.0 & $N$ & 24.3 \\
\hline $7,1,120-122$ & 54.11 & 20.30 & +76.6 & 234.1 & +82.9 & 220.7 & $\mathrm{~N}$ & 21.8 \\
\hline $7,2,27-29$ & 54.68 & 8.62 & +69.3 & 222.4 & +68.7 & 224.5 & $\mathrm{~N}$ & 24.9 \\
\hline $7,2,49-51$ & 54.90 & 1.41 & +85.8 & 314.6 & +63.1 & 189.6 & $\mathrm{~N}$ & 7.6 \\
\hline $7,2,70-72$ & 55.11 & 1.50 & +60.5 & 131.3 & +68.0 & 184.1 & $\mathrm{~N}$ & 7.1 \\
\hline $7,2,107-109$ & 55.48 & .70 & +83.2 & 49.6 & +67.4 & 193.6 & $\mathrm{~N}$ & 14.0 \\
\hline $7,3,13-15$ & 56.04 & 21.17 & +68.8 & 240.1 & +74.6 & 227.0 & $\mathrm{~N}$ & 37.4 \\
\hline $7,3,47-49$ & 56.38 & 15.70 & +75.1 & 217.6 & +75.7 & 226.5 & $\mathrm{~N}$ & 30.9 \\
\hline $7,3,72-74$ & 56.63 & 24.59 & +77.7 & 203.4 & +80.5 & 219.1 & $N$ & 45.2 \\
\hline $7,3,105-107$ & 56.96 & 13.97 & +79.7 & 189.0 & +79.3 & 199.9 & $\mathrm{~N}$ & 36.3 \\
\hline $7,4,12-14$ & 57.53 & 5.37 & +70.7 & 222.1 & +71.9 & 216.1 & $\mathrm{~N}$ & 29.8 \\
\hline $7,4,47-49$ & 57.88 & 6.97 & +80.4 & 174.3 & +83.0 & 191.3 & $\mathrm{~N}$ & 32.5 \\
\hline $7,4,82-84$ & 58.23 & 58.82 & +74.1 & 265.7 & +77.6 & 216.1 & $\mathrm{~N}$ & 41.6 \\
\hline $8,1,33-35$ & 64.04 & 15.16 & +44.2 & 80.6 & +73.9 & 107.9 & $\mathrm{~N}$ & 33.6 \\
\hline $8,1,69-71$ & 64.40 & 16.68 & +81.3 & 123.0 & +81.6 & 168.4 & $\mathrm{~N}$ & 21.9 \\
\hline $8,1,109-111$ & 64.80 & 28.20 & +80.3 & 233.8 & +78.2 & 232.5 & $\mathrm{~N}$ & 23.4 \\
\hline
\end{tabular}




\begin{tabular}{|c|c|c|c|c|c|c|c|c|}
\hline $\begin{array}{l}\text { Core, Section, } \\
\text { Interval }(\mathrm{cm})\end{array}$ & $\begin{array}{l}\text { Depth } \\
\text { (mbsf) }\end{array}$ & $\begin{array}{c}\underset{\mathrm{JRM}}{\mathrm{J}} \\
(\mathrm{mA} / \mathrm{m})\end{array}$ & $\begin{array}{l}\text { Incl. } \\
\text { NRM } \\
\text { (deg) }\end{array}$ & $\begin{array}{l}\text { Decl. } \\
\text { NRM } \\
\text { (deg) }\end{array}$ & $\begin{array}{c}\text { Incl } \\
\text { stable } \\
\text { (deg) }\end{array}$ & $\begin{array}{l}\text { Decl. } \\
\text { stable } \\
\text { (deg) }\end{array}$ & Pol. & $\begin{array}{l}\text { MDF } \\
(\mathrm{mT})\end{array}$ \\
\hline $8,2,34-36$ & 65.55 & 17.69 & +84.5 & 45.7 & +83.8 & 30.2 & $\mathrm{~N}$ & 15.8 \\
\hline $8,2,85-87$ & 66.06 & 137.14 & +85.1 & -58.7 & +76.6 & 44.3 & $\mathrm{~N}$ & 7.6 \\
\hline $8,2,134-1.36$ & 66.55 & 15.11 & +58.7 & 77.6 & +78.9 & 71.1 & $\mathrm{~N}$ & 30.9 \\
\hline $8,3,34-36$ & 67.05 & 5.87 & +79.5 & 80.0 & +79.3 & 94.7 & $\mathrm{~N}$ & 27.0 \\
\hline $8,3,84-86$ & 67.55 & 7.48 & +79.1 & 127.8 & +70.5 & 106.3 & $\mathrm{~N}$ & 32.9 \\
\hline $8,3,135-137$ & 68.06 & 11.08 & +72.9 & 111.9 & +75.2 & 114.2 & $\mathrm{~N}$ & 27.9 \\
\hline $8,4,19-21$ & 68.40 & 47.90 & +36.8 & 88.6 & +71.8 & 76.5 & $\mathrm{~N}$ & 7.4 \\
\hline $8,5,19-21$ & 69.90 & 21.16 & +85.3 & 21.3 & +82.5 & 190.2 & $\mathrm{~N}$ & 26.3 \\
\hline $8,5,83-85$ & 70.54 & 39.38 & +72.6 & 213.8 & +72.1 & 216.0 & $\mathrm{~N}$ & 35.8 \\
\hline $8,5,134-136$ & 71.05 & 12.83 & +70.1 & 235.1 & +71.3 & 216.5 & $\mathrm{~N}$ & 29.1 \\
\hline $8,6,19-21$ & 71.40 & 23.89 & +72.8 & 155.5 & +69.9 & 155.1 & $\mathrm{~N}$ & 8.6 \\
\hline $8, C, 13-15$ & 71.64 & 21.51 & +80.2 & 216.8 & +80.8 & 241.8 & $\mathrm{~N}$ & 20.4 \\
\hline $8,6,60-62$ & 71.81 & 7.60 & +73.5 & 10.1 & +72.0 & 11.1 & $\mathrm{~N}$ & 17.6 \\
\hline $8,7,13-15$ & 72.84 & 48.63 & +71.8 & 98.3 & +73.3 & 98.6 & $\mathrm{~N}$ & 23.7 \\
\hline $9,1,35-37$ & 73.56 & 24.77 & +85.1 & 169.2 & +88.4 & 27 & $\mathrm{~N}$ & 22.7 \\
\hline $9,1,85-87$ & 74.06 & 12.52 & +89.1 & 130.4 & +8 & 29 & $\mathrm{~N}$ & 12.7 \\
\hline $9,1,135-137$ & 74.56 & 4.76 & +84.0 & 291.1 & +74.5 & 305.7 & $\mathrm{~N}$ & 14.7 \\
\hline $9,2,35-37$ & 75.06 & 2.98 & +84.2 & 248.2 & +81.1 & 306.2 & $\mathrm{~N}$ & 9.9 \\
\hline $9,2,85-87$ & 75.56 & 4.28 & +62.9 & 100.8 & +81.4 & 268.4 & $\mathrm{~N}$ & 15.9 \\
\hline $9,2,135-137$ & 76.06 & 23.66 & +73.8 & 246.1 & .5 & 259.8 & $\mathrm{~N}$ & 19.7 \\
\hline $9,3,35-37$ & 76.56 & 12.34 & +84.2 & 290.0 & .9 & 297.4 & $\mathrm{~N}$ & 18.4 \\
\hline $9,3,85-87$ & 77.06 & 28.44 & +76.5 & 253.4 & +75.3 & 255.8 & $\mathrm{~N}$ & 18.5 \\
\hline $9,3,135-137$ & 77.56 & 8.61 & +88.2 & 167.7 & +87.0 & 282.1 & $\mathrm{~N}$ & 20.1 \\
\hline $9,4,35-37$ & 78.06 & 35.99 & +79.0 & 244.2 & +77.8 & 250.5 & $\mathrm{~N}$ & 24.7 \\
\hline $9,4,85-87$ & 78.56 & 53.90 & +81.4 & 252.7 & +77.9 & 257.8 & $\mathrm{~N}$ & 25.6 \\
\hline $9,4,103-105$ & 78.74 & 51.55 & +85.9 & 254.3 & +84.0 & 273.5 & $\mathrm{~N}$ & 24.3 \\
\hline $9,5,35-37$ & 79.56 & 15.04 & +74.1 & 133.1 & +72.7 & 243.5 & $\mathrm{~N}$ & 18.7 \\
\hline $9,5,85-87$ & 80.06 & 3.11 & +87.7 & 153.8 & +75.8 & 240.2 & $\mathrm{~N}$ & 41.5 \\
\hline $9,5,135-137$ & 80.56 & 1.36 & +75.6 & 202.0 & +85.6 & 228.3 & $\mathrm{~N}$ & 18.8 \\
\hline $9,6,13-15$ & 80.84 & .64 & +69.1 & 105.6 & +68.5 & 89.7 & $\mathrm{~N}$ & 17.6 \\
\hline $10,3,35-37$ & 86.06 & 8.34 & -78.2 & 53.0 & -78.5 & 24.8 & $\mathrm{R}$ & 27.0 \\
\hline $10,3,85-87$ & 86.56 & 22.04 & -74.8 & 93.0 & -81.5 & 47.1 & $\mathrm{R}$ & 26.8 \\
\hline $10,3,135-137$ & 87.06 & 24.82 & -73.5 & 62.4 & -72.6 & 38.1 & $\mathrm{R}$ & 26.0 \\
\hline $10,4,35-37$ & 87.56 & 2.83 & -59.9 & 91.9 & -70.6 & 47.0 & $\mathrm{R}$ & 40.8 \\
\hline $10,4,85-87$ & 88.06 & 3.56 & -73.1 & 14.3 & -67.6 & 16.9 & $\mathrm{R}$ & 21.5 \\
\hline $10,4,135-137$ & 88.56 & 2.95 & -62.1 & 22.9 & -74.8 & 28.8 & $\mathrm{R}$ & 23.4 \\
\hline $10,5,35-37$ & 89.06 & 33.05 & -70.5 & 10.0 & -69.7 & 13.7 & $\mathrm{R}$ & 55.2 \\
\hline $10,5,85-87$ & 89.56 & 4.60 & -70.4 & 64.0 & -71.9 & 2.6 & $\mathrm{R}$ & 51.2 \\
\hline $10,5,135-137$ & 90.06 & 7.25 & -62.7 & 63.9 & -72.7 & 43.3 & $\mathrm{R}$ & 57.9 \\
\hline $11,1,34-36$ & o & 22. & -6 & 20 & & & $\mathrm{R}$ & 3.0 \\
\hline $11,1,85-87$ & 93.06 & 2.55 & +87.8 & 148.5 & -6 & 134.2 & $\mathrm{R}$ & 3.2 \\
\hline $11,1,135-137$ & 93.56 & 9.36 & -56.8 & 78.6 & -74.8 & 90.1 & $\mathrm{R}$ & 32.4 \\
\hline $11,2,34-36$ & 94.05 & 15.29 & -69.6 & 155.9 & -76.5 & 162.2 & $\mathrm{R}$ & 29.8 \\
\hline $11,2,85-87$ & 94.56 & 34.19 & -79.8 & 173.2 & -82.0 & 167.0 & $\mathrm{R}$ & 28.0 \\
\hline $11,2,135-137$ & 95.06 & 3.79 & -33.8 & 167.6 & -66.6 & 181.3 & $\mathrm{R}$ & 24.6 \\
\hline $11,3,34-36$ & 95.55 & .10 & -74.8 & 241.4 & -87.2 & 215.7 & $\mathrm{R}$ & \\
\hline $11,3,85-87$ & 96.06 & .25 & -19.7 & 338.1 & -71.3 & 210.7 & $\mathrm{R}$ & \\
\hline
\end{tabular}


Table A5 (continued).

\begin{tabular}{|c|c|c|c|c|c|c|c|c|}
\hline $\begin{array}{l}\text { Core, Section, } \\
\text { Interval }(\mathrm{cm})\end{array}$ & $\begin{array}{l}\text { Depth } \\
\text { (mbsf) }\end{array}$ & $\begin{array}{c}\mathrm{J} \\
\mathrm{NRM} \\
(\mathrm{mA} / \mathrm{m})\end{array}$ & $\begin{array}{l}\text { Incl. } \\
\text { NRM } \\
\text { (deg) }\end{array}$ & $\begin{array}{l}\text { Decl. } \\
\text { NRM } \\
\text { (deg) }\end{array}$ & $\begin{array}{l}\text { Incl } \\
\text { stable } \\
\text { (deg) }\end{array}$ & $\begin{array}{l}\text { Decl. } \\
\text { stable } \\
\text { (deg) }\end{array}$ & Pol. & $\begin{array}{l}\mathrm{MDF} \\
(\mathrm{mT})\end{array}$ \\
\hline $11,3,135-137$ & 96.56 & 1.54 & -85.4 & 209.3 & -87.2 & 206.6 & R & 57.0 \\
\hline $11,4,34-36$ & 97.05 & 13.73 & +83.1 & 263.9 & -72.3 & 216.9 & R & 4.2 \\
\hline $11,4,85-87$ & 97.56 & 19.13 & -80.6 & 181.1 & -82.6 & 210.3 & $\mathrm{R}$ & 50.0 \\
\hline $11,4,135-137$ & 98.06 & 12.72 & -86.6 & 106.7 & -89.2 & 203.3 & $\mathrm{R}$ & 43.9 \\
\hline $11,5,34-36$ & 98.55 & 1.04 & -8.0 & 239.3 & -67.1 & 242.7 & $\mathrm{R}$ & \\
\hline $11,5,85-87$ & 99.06 & 17.92 & -16.1 & 92.7 & -76.2 & 228.4 & $\mathrm{R}$ & 20.0 \\
\hline $11,5,135-137$ & 99.56 & 5.11 & -54.9 & 180.3 & -75.6 & 222.3 & $\mathrm{R}$ & 45.3 \\
\hline $11,6,34-36$ & 100.05 & 10.52 & -54.9 & 189.3 & -69.1 & 182.0 & $\mathrm{R}$ & 28.5 \\
\hline $11,6,85-87$ & 100.56 & 6.31 & -77.8 & 156.2 & -82.7 & 201.9 & R & 29.9 \\
\hline $11,6,135-137$ & 101.06 & 2.12 & +66.1 & 169.1 & -74.8 & 232.0 & $\mathrm{R}$ & 2.8 \\
\hline $11,7,18-20$ & 101.39 & 1.58 & +66.4 & 292.8 & -78.6 & 228.2 & $\mathrm{R}$ & 3.5 \\
\hline $11,7,50-52$ & 101.71 & 1.13 & +85.9 & 309.4 & -77.4 & 122.8 & $\mathrm{R}$ & 3.2 \\
\hline $12,1,35-37$ & 102.06 & 10.47 & -10.9 & 111.7 & - & 35.1 & $\mathrm{R}$ & 21.5 \\
\hline $12,1,84-86$ & 102.55 & 3.42 & -56.6 & 38.1 & -66.5 & 19.9 & $\mathrm{R}$ & 33.0 \\
\hline $12,1,135-137$ & 103.06 & 6.21 & -66.2 & 83.0 & -73.8 & 59.8 & $\mathrm{R}$ & 23.6 \\
\hline $12,2,35-37$ & 103.56 & 19.31 & -81.8 & 60.1 & -83.1 & 63.1 & $\mathrm{R}$ & 27.8 \\
\hline $12,2,43-45$ & 103.64 & 7.92 & -60.0 & 55.5 & -72.2 & 29.1 & $\mathrm{R}$ & 24.7 \\
\hline $12,2,51-53$ & 103.72 & 4.11 & -29.0 & 112.8 & -56.5 & 102.1 & $\mathrm{R}$ & 17.9 \\
\hline $12,2,57-59$ & 103.78 & 2.04 & +17.8 & 104.7 & -27.9 & 118.9 & & 14.2 \\
\hline $12,2,64-66$ & 103.85 & 1.30 & +34.1 & 37.2 & -43.6 & 7.1 & & 39.0 \\
\hline $12,2,72-74$ & 103.93 & .67 & +54.1 & 120.1 & +44.6 & 102.6 & & 6.1 \\
\hline $12,2,77-79$ & 103.98 & 1.36 & +7.7 & 103.3 & +2.4 & 109.8 & & 6.9 \\
\hline $12,2,85-87$ & 104.06 & 6.56 & +63.2 & 141.8 & +76.9 & 139.5 & $\mathrm{~N}$ & 12.8 \\
\hline $12,2,135-137$ & 104.56 & 11.30 & +82.2 & 134.5 & +84.9 & 139.3 & $\mathrm{~N}$ & 20.0 \\
\hline $12,3,35-37$ & 105.06 & 35.82 & +51.4 & 203.0 & +76.1 & 176.3 & $\mathrm{~N}$ & 13.0 \\
\hline $12,3,85-87$ & 105.56 & 18.03 & +76.1 & 165.4 & +78.6 & 157.8 & $N$ & 20.2 \\
\hline $12,3,135-137$ & 106.06 & 16.70 & +76.4 & 164.5 & +78.3 & 159.8 & $\mathrm{~N}$ & 19.6 \\
\hline $12,4,34-36$ & 106.55 & 9.65 & +74.7 & 170.4 & +83.5 & 174.8 & $\mathrm{~N}$ & 16.6 \\
\hline $12,4,84-86$ & 107.05 & 8.78 & +70.8 & 142.8 & +78.9 & 135.5 & $\mathrm{~N}$ & 17.1 \\
\hline $12,4,114-116$ & 107.35 & 11.27 & +76.4 & 164.4 & +76.7 & 146.6 & $\mathrm{~N}$ & 15.2 \\
\hline $12,5,35-37$ & 108.06 & 22.77 & +80.4 & 154.6 & +85.0 & 146.1 & $\mathrm{~N}$ & 19.8 \\
\hline $12,5,85-87$ & 108.56 & 18.20 & +67.4 & 142.9 & +67.2 & 135.8 & $\mathrm{~N}$ & 20.1 \\
\hline $12,5,135-137$ & 109.06 & 4.15 & +78.0 & 159.2 & +78.4 & 155.5 & $\mathrm{~N}$ & 22.0 \\
\hline $12,6,35-37$ & 109.56 & 5.86 & +77.7 & 185.4 & +82.7 & 145.7 & $\mathrm{~N}$ & 19.9 \\
\hline $12,6,85-87$ & 110.06 & 2.24 & +89.1 & 89.6 & +83.4 & 141.3 & $\mathrm{~N}$ & 19.5 \\
\hline $12,6,135-137$ & 110.56 & .23 & +46.0 & 130.4 & +52.7 & 131.4 & $\mathrm{~N}$ & 13.2 \\
\hline $12,7,27-29$ & 110.98 & .18 & +20.1 & 124.4 & +24.3 & 135.3 & & 14.0 \\
\hline $12,7,75-77$ & 111.46 & .15 & +18.9 & 134.5 & -5.0 & 154.5 & & 10.5 \\
\hline $13,1,58-60$ & 111.79 & .1 & -14.8 & & -79.3 & & $\mathrm{R}$ & \\
\hline $13,1,85-87$ & 112.06 & .9 & +32.8 & 83.1 & -67.6 & 82.2 & $\mathrm{R}$ & 18.2 \\
\hline $13,1,135-137$ & 112.56 & 3.7 & +24.6 & 132.7 & -63.1 & 117.4 & $\mathrm{R}$ & 28.5 \\
\hline $13,2,36-38$ & 113.07 & 1.77 & +66.4 & 130.6 & -66.6 & 117.2 & $\mathrm{R}$ & 6.0 \\
\hline $13,2,85-87$ & 113.56 & 3.78 & -70.3 & .7 & -67.0 & 17.2 & R & 33.8 \\
\hline $13,2,135-137$ & 114.06 & 1.46 & -50.4 & 89.8 & -66.4 & 43.7 & $\mathrm{R}$ & 41.9 \\
\hline $13,3,45-47$ & 114.66 & 19.41 & -58.3 & 79.8 & -61.1 & 84.8 & $\mathrm{R}$ & 26.8 \\
\hline $13,4,25-27$ & 115.96 & 16.07 & -67.6 & 124.2 & -65.0 & 119.7 & $\mathrm{R}$ & 23.0 \\
\hline $13,4,79-81$ & 116.50 & 4.73 & -79.2 & 138.4 & -81.4 & 127.5 & R & 22.7 \\
\hline $13,4,125-127$ & 116.96 & .31 & -46.8 & 132.5 & -71.9 & 266.6 & $\mathrm{R}$ & 9.8 \\
\hline
\end{tabular}


Table A5 (continued).

\begin{tabular}{|c|c|c|c|c|c|c|c|c|}
\hline $\begin{array}{l}\text { Core, Section, } \\
\text { Interval }(\mathrm{cm})\end{array}$ & $\begin{array}{l}\text { Depth } \\
\text { (mbsf) }\end{array}$ & $\begin{array}{c}\mathrm{J} \\
\mathrm{NRM} \\
(\mathrm{mA} / \mathrm{m})\end{array}$ & $\begin{array}{l}\text { Incl. } \\
\text { NRM } \\
\text { (deg) }\end{array}$ & $\begin{array}{l}\text { Decl. } \\
\text { NRM } \\
\text { (deg) }\end{array}$ & $\begin{array}{c}\text { Incl } \\
\text { stable } \\
\text { (deg) }\end{array}$ & $\begin{array}{l}\text { Decl. } \\
\text { stable } \\
\text { (deg) }\end{array}$ & Pol. & $\begin{array}{l}\text { MDF } \\
(\mathrm{mT})\end{array}$ \\
\hline $13,5,37-39$ & 117.58 & $£ .31$ & +40.3 & 142.7 & -63.8 & 159.6 & R & 4.0 \\
\hline $13,5,77-79$ & 117.98 & 3.14 & +76.8 & 127.8 & -72.6 & 359.1 & $\mathrm{R}$ & 16.0 \\
\hline $13,6,18-20$ & 118.89 & 8.73 & -64.3 & 118.7 & -72.0 & 161.8 & $\mathrm{R}$ & 4.9 \\
\hline $13,6,77-79$ & 119.48 & 2.94 & +38.9 & 281.2 & -82.8 & 346.4 & $\mathrm{R}$ & 3.2 \\
\hline $13,7,33-35$ & 120.54 & .92 & +74.7 & 30.7 & -62.9 & 35.9 & $\mathrm{R}$ & 7.5 \\
\hline $13,7,84-86$ & 121.05 & 9.46 & -62.3 & 132.4 & -65.4 & 123.2 & $\mathrm{R}$ & 3.9 \\
\hline $13,7,133-135$ & 121.54 & .37 & -62.8 & 69.0 & -62.6 & 76.6 & $\mathrm{R}$ & 5.0 \\
\hline $14,2,85-87$ & 123.06 & 60.02 & -4.7 & 124.4 & -63.3 & 112.1 & $\mathrm{R}$ & 4.9 \\
\hline $14,2,135-137$ & 123.56 & 5.93 & -83.8 & 158.4 & -75.3 & 262.7 & $\mathrm{R}$ & 39.9 \\
\hline $14,3,35-37$ & 124.06 & 12.10 & +76.4 & 138.1 & +70.1 & 104.6 & & 10.8 \\
\hline $14,3,85-87$ & 124.56 & 44.78 & -58.5 & 77.9 & -62.5 & 74.2 & R & 27.0 \\
\hline $14,4,35-37$ & 125.56 & 19.46 & -60.6 & 103.7 & -71.0 & 85.8 & $\mathrm{R}$ & 25.1 \\
\hline $14,4,85-87$ & 126.06 & 1.06 & -56.9 & 317.2 & -63.7 & 20.3 & $\mathrm{R}$ & 3.8 \\
\hline $14,5,35-37$ & 127.06 & 3.52 & -61.7 & 79.4 & -64.4 & 77.9 & $\mathrm{R}$ & 45.7 \\
\hline $14,5,85-87$ & 127.56 & 4.50 & -46.0 & 23.2 & -67.8 & 33.6 & $\mathrm{R}$ & 23.7 \\
\hline $14,5,135-137$ & 128.06 & 2.39 & +9.7 & 104.8 & -78.2 & 68.3 & $\mathrm{R}$ & 34.6 \\
\hline $15,1,35-37$ & 130.56 & 3.00 & -61.1 & 132.5 & .9 & 190.6 & $\mathrm{R}$ & 3.5 \\
\hline $15,1,85-87$ & 131.06 & 3.69 & +43.6 & 297.9 & -77.1 & 259.0 & $\mathrm{R}$ & 3.0 \\
\hline $15,1,135-137$ & 131.56 & .67 & +68.1 & 244.3 & -73.3 & 249.2 & $\mathrm{R}$ & 44.2 \\
\hline $15,2,35-37$ & 132.06 & .13 & -12.1 & 161.8 & -83.5 & 230.6 & $\mathrm{R}$ & \\
\hline $15,2,85-87$ & 132.56 & 10.37 & +66.0 & 111.3 & -81.0 & 241.0 & $\mathrm{R}$ & 3.7 \\
\hline $15,2,135-137$ & 133.06 & .09 & -23.6 & 35.0 & -87.2 & 258.5 & $\mathrm{R}$ & \\
\hline $15,3,85-87$ & 134.06 & .13 & +67.6 & .3 & -70.9 & 243.2 & $\mathrm{R}$ & 8.0 \\
\hline $15,3,135-137$ & 134.56 & .33 & +49.8 & 346.8 & -67.9 & 242.2 & $\mathrm{R}$ & 35.4 \\
\hline $15,4,35-37$ & 135.06 & .43 & -60.6 & 308.5 & -71.2 & 291.9 & $\mathrm{R}$ & 3.2 \\
\hline $15,4,85-87$ & 135.56 & .59 & -43.2 & 57.2 & -68.8 & 19.1 & $\mathrm{R}$ & 65.4 \\
\hline $15,5,35-37$ & 136.56 & .39 & +2.0 & 119.4 & -61.6 & 141.5 & $\mathrm{R}$ & 2.3 \\
\hline $15,5,85-87$ & 137.06 & .14 & -13.5 & 81.5 & -77.2 & 137.3 & $\mathrm{R}$ & \\
\hline $15,5,135-137$ & 137.56 & 1.82 & +46.1 & 106.7 & -70.4 & 146.9 & $\mathrm{R}$ & 2.6 \\
\hline $15,6,35-37$ & 138.06 & .66 & -63.0 & 72.1 & -64.3 & 56.8 & $\mathrm{R}$ & 6.1 \\
\hline $15,7,35-37$ & 139.56 & 5.20 & +33.6 & 132.6 & -62.6 & 56.3 & $\mathrm{R}$ & 2.6 \\
\hline $16,1,85-87$ & 140.56 & 1.17 & +84.9 & 95.6 & -61.6 & 92.5 & $\mathrm{R}$ & 2.1 \\
\hline $16,1,135-137$ & 141.06 & 1.34 & +76.8 & 128.2 & -69.2 & 64.0 & $\mathrm{R}$ & 2.7 \\
\hline $16,2,35-37$ & 141.56 & 8.18 & +85.4 & 117.7 & -67.6 & 40.5 & $\mathrm{R}$ & 4.4 \\
\hline $16,2,85-87$ & 142.06 & 3.00 & +81.9 & 344.3 & .72 .1 & 63.9 & $\mathrm{R}$ & 2.3 \\
\hline $16,2,135-137$ & 142.56 & .57 & +41.6 & 85.1 & -81.7 & 31.3 & $\mathrm{R}$ & 69.7 \\
\hline $16,3,35-37$ & 143.06 & .40 & +24.8 & 73.9 & -70.4 & 55.7 & $\mathrm{R}$ & 49.4 \\
\hline $16,3,85-87$ & 143.56 & 6.99 & +83.3 & 357.5 & -62.3 & 34.6 & $\mathrm{R}$ & 2.9 \\
\hline $16,3,135-137$ & 144.06 & 1.33 & +29.0 & 27.8 & -66.0 & 18.8 & $\mathrm{R}$ & 2.7 \\
\hline $16,4,35-37$ & 144.56 & .74 & +80.1 & 105.9 & -65.6 & 41.2 & $\mathrm{R}$ & 2.5 \\
\hline $16,4,85-87$ & 145.06 & .18 & +82.3 & 266.5 & -68.2 & 68.4 & $\mathrm{R}$ & 1.8 \\
\hline $16,4,135-137$ & 145.56 & .24 & +43.6 & 88.1 & -66.1 & 21.0 & $\mathrm{R}$ & 35.5 \\
\hline $16,5,35-37$ & 146.06 & .24 & -1.5 & 244.8 & -63.0 & 10.7 & $\mathrm{R}$ & \\
\hline $16,5,85-87$ & 146.56 & .38 & +76.1 & 84.3 & -63.9 & 53.9 & $\mathrm{R}$ & 3.1 \\
\hline $16,5,135-137$ & 147.06 & .20 & +61.3 & 11.7 & -71.6 & 55.1 & R & 4.5 \\
\hline $16,6,35-37$ & 147.56 & .96 & +77.2 & 216.6 & -70.4 & 4.3 & $\mathrm{R}$ & 3.1 \\
\hline
\end{tabular}


Table A5 (continued).

\begin{tabular}{|c|c|c|c|c|c|c|c|c|}
\hline $\begin{array}{l}\text { Core, Section, } \\
\text { Interval }(\mathrm{cm})\end{array}$ & $\begin{array}{l}\text { Depth } \\
\text { (mbsf) }\end{array}$ & $\begin{array}{c}\mathrm{J} \\
\mathrm{NRM} \\
(\mathrm{mA} / \mathrm{m})\end{array}$ & $\begin{array}{l}\text { Incl. } \\
\text { NRM } \\
\text { (deg) }\end{array}$ & $\begin{array}{l}\text { Decl. } \\
\text { NRM } \\
\text { (deg) }\end{array}$ & $\begin{array}{c}\text { Incl } \\
\text { stable } \\
\text { (deg) }\end{array}$ & $\begin{array}{l}\text { Decl. } \\
\text { stable } \\
\text { (deg) }\end{array}$ & Pol. & $\begin{array}{l}\mathrm{MDF} \\
(\mathrm{mT})\end{array}$ \\
\hline $17,1,35-37$ & 149.56 & .36 & +50.7 & 11.2 & -72.0 & 61.2 & $\mathrm{R}$ & \\
\hline $17,1,135-137$ & 150.56 & .24 & -23.9 & 120.2 & -60.1 & 104.2 & $\mathrm{R}$ & 26.6 \\
\hline $17,2,31-33$ & 151.02 & .08 & +2.3 & 72.2 & -61.2 & 62.8 & $\mathrm{R}$ & 13.1 \\
\hline $17,2,56-58$ & 151.27 & .27 & -60.5 & 82.7 & -65.0 & 50.4 & $\mathrm{R}$ & 3.8 \\
\hline $17,3,35-37$ & 152.56 & .20 & -27.5 & 95.8 & -65.9 & 74.0 & $\mathrm{R}$ & \\
\hline $17,3,56-58$ & 152.77 & .18 & -32.6 & 79.1 & -62.4 & 54.1 & $\mathrm{R}$ & 29.8 \\
\hline $17,4,37-39$ & 154.08 & .13 & +45.2 & 148.2 & -73.2 & 34.9 & $\mathrm{R}$ & 3.8 \\
\hline $17,4,95-97$ & 154.66 & .19 & -33.3 & 121.4 & -83.9 & 85.3 & $\mathrm{R}$ & 5.3 \\
\hline $17,4,133-135$ & 155.04 & .23 & -19.5 & 115.1 & -58.7 & 90.1 & $R$ & 10.4 \\
\hline $17,5,35-37$ & 155.56 & .14 & -17.3 & 57.0 & -67.2 & 78.5 & $\mathrm{R}$ & \\
\hline $17,5,117-119$ & 156.38 & .53 & -68.3 & 90.4 & -75.1 & 96.5 & $\mathrm{R}$ & 5.9 \\
\hline $17,6,49-51$ & 157.20 & .20 & +15.7 & 157.2 & -64.8 & 89.2 & $R$ & 66.0 \\
\hline $17,6,85-87$ & 157.56 & .49 & +60.6 & 202.4 & -70.6 & 115.2 & $\mathrm{R}$ & 60.6 \\
\hline $17,7,23-25$ & 158.44 & .29 & -33.1 & 87.0 & -74.8 & 54.0 & $\mathrm{k}$ & 97.4 \\
\hline $18,1,35-37$ & 159.06 & .28 & -61.7 & 101.1 & -68.8 & 124.2 & $\mathrm{R}$ & 35.1 \\
\hline $18,1,95-97$ & 159.66 & .14 & -50.5 & 108.4 & -79.0 & 149.7 & $\mathrm{R}$ & \\
\hline $18,1,135-137$ & 160.06 & .19 & +27.9 & 125.3 & -71.2 & 114.8 & $\mathrm{R}$ & 4.8 \\
\hline $18,2,35-37$ & 160.56 & .45 & -16.3 & 109.9 & -62.7 & 134.2 & $\mathrm{R}$ & 54.0 \\
\hline $18,2,95-97$ & 161.16 & 1.25 & +5.9 & 143.4 & -75.3 & 135.2 & $\mathrm{R}$ & 35.1 \\
\hline $18,2,135-137$ & 161.56 & .31 & +8.7 & 136.4 & -74.0 & 123.1 & $\mathrm{R}$ & 42.9 \\
\hline $18,3,35-37$ & 162.06 & .71 & -12.4 & 173.7 & -68.6 & 131.4 & $\mathrm{R}$ & 20.5 \\
\hline $18,3,95-97$ & 162.66 & .26 & -58.1 & 107.3 & -69.6 & 105.0 & $\mathrm{R}$ & \\
\hline $18,3,135-137$ & 163.06 & .69 & +58.2 & 198.1 & -62.8 & 107.7 & $\mathrm{R}$ & 10.3 \\
\hline $18,4,35-37$ & 163.56 & .68 & +33.1 & 134.2 & -74.8 & 113.7 & $\mathrm{R}$ & 25.5 \\
\hline $18,4,95-97$ & 164.16 & .37 & +50.5 & 147.9 & -62.6 & 136.5 & $\mathrm{R}$ & 43.0 \\
\hline $18,5,35-37$ & 165.06 & .15 & -35.0 & 92.6 & -65.0 & 125.7 & $\mathrm{R}$ & \\
\hline $18,5,95-97$ & 165.66 & .07 & -64.3 & 71.7 & -76.2 & 152.7 & $\mathrm{R}$ & \\
\hline $18,5,135-137$ & 166.06 & .08 & +53.7 & 60.4 & -62.7 & 142.7 & $\mathrm{R}$ & 4.1 \\
\hline $18,6,35-37$ & 166.56 & .18 & +65.6 & 28.9 & -66.3 & 205.0 & $\mathrm{R}$ & 7.9 \\
\hline $18,6,93-95$ & 167.14 & .28 & +64.4 & 345.4 & +64.4 & 345.4 & $\mathrm{~N}$ & 20.0 \\
\hline $18,7,35-37$ & 168.06 & .53 & +75.9 & 155.4 & +68.8 & 314.6 & $\mathrm{~N}$ & 4.3 \\
\hline $19,3,9-11$ & 171.30 & .36 & +56.8 & 118.2 & +66.9 & 110.6 & $\mathrm{~N}$ & \\
\hline $19,4,35-37$ & 173.06 & 1.03 & +66.5 & 156.0 & +74.7 & 160.1 & $\mathrm{~N}$ & 34.6 \\
\hline $19,4,85-87$ & 173.56 & .33 & +49.6 & 151.1 & +73.2 & 140.3 & $\mathrm{~N}$ & 30.3 \\
\hline $19,4,130-132$ & 174.01 & .29 & +42.0 & 142.5 & +68.0 & 164.9 & $\mathrm{~N}$ & 25.8 \\
\hline $19,5,35-37$ & 174.56 & .14 & +51.7 & 150.2 & +68.6 & 171.6 & $\mathrm{~N}$ & 38.4 \\
\hline $19,5,85-87$ & 175.06 & .19 & +43.4 & 116.3 & +54.0 & 130.6 & $\mathrm{~N}$ & 44.3 \\
\hline $19,5,135-137$ & 175.56 & .17 & +63.5 & 135.0 & +67.3 & 144.9 & $\mathrm{~N}$ & 23.0 \\
\hline $20,1,35-37$ & 178.06 & .65 & +84.1 & 333.7 & +81.0 & 152.1 & $\mathrm{~N}$ & 23.8 \\
\hline $20,1,85-87$ & 178.56 & 1.36 & +81.5 & 68.5 & +64.1 & 156.0 & $\mathrm{~N}$ & 6.4 \\
\hline $20,1,135-137$ & 179.06 & .48 & +75.9 & 148.9 & +77.7 & 198.3 & $\mathrm{~N}$ & 29.7 \\
\hline $20,2,35-37$ & 179.56 & 1.23 & +80.1 & 202.5 & +74.9 & 208.5 & $\mathrm{~N}$ & 27.7 \\
\hline $20,2,85-87$ & 180.06 & .23 & +68.5 & 83.3 & +68.4 & 173.6 & $\mathrm{~N}$ & 15.1 \\
\hline $20,2,135-137$ & 180.56 & .47 & +72.2 & 121.0 & +70.3 & 205.0 & $\mathrm{~N}$ & 11.0 \\
\hline $20,3,35-37$ & 181.06 & .26 & -14.4 & 88.8 & -76.0 & 89.4 & $\mathrm{R}$ & \\
\hline $20,3,85-87$ & 181.56 & .28 & +35.0 & 83.7 & -63.4 & 78.6 & $\mathrm{R}$ & 44.2 \\
\hline $20,3,135-137$ & 182.06 & .35 & -57.7 & 9.0 & -67.0 & 22.1 & $\mathrm{R}$ & 66.9 \\
\hline
\end{tabular}


Table A5 (continued).

\begin{tabular}{|c|c|c|c|c|c|c|c|c|}
\hline $\begin{array}{l}\text { Core, Section, } \\
\text { Interval }(\mathrm{cm})\end{array}$ & $\begin{array}{l}\text { Depth } \\
\text { (mbsf) }\end{array}$ & $\begin{array}{c}\mathrm{J} \\
\mathrm{NRM} \\
(\mathrm{mA} / \mathrm{m})\end{array}$ & $\begin{array}{l}\text { Incl. } \\
\text { NRM } \\
\text { (deg) }\end{array}$ & $\begin{array}{l}\text { Decl. } \\
\text { NRM } \\
\text { (deg) }\end{array}$ & $\begin{array}{l}\text { Incl } \\
\text { stable } \\
\text { (deg) }\end{array}$ & $\begin{array}{l}\text { Decl. } \\
\text { stable } \\
\text { (deg) }\end{array}$ & Pol. & $\begin{array}{l}\text { MDF } \\
(\mathrm{mT})\end{array}$ \\
\hline $\begin{array}{ll}20,4, & 35-37 \\
20,4, & 85-87 \\
20,5, & 34-36 \\
20,5, & 84-86\end{array}$ & $\begin{array}{l}182.56 \\
183.06 \\
184.05 \\
184.55\end{array}$ & $\begin{array}{l}.19 \\
.22 \\
.75 \\
.30\end{array}$ & $\begin{array}{l}+52.6 \\
+38.9 \\
-55.6 \\
-38.2\end{array}$ & $\begin{array}{l}13.3 \\
68.8 \\
49.9 \\
72.6\end{array}$ & $\begin{array}{l}-64.3 \\
-69.3 \\
-68.8 \\
-72.9\end{array}$ & $\begin{array}{l}17.5 \\
51.0 \\
31.5 \\
53.6\end{array}$ & $\begin{array}{l}R \\
R \\
R \\
R\end{array}$ & 7.8 \\
\hline $\begin{array}{l}21,1, \quad 35-37 \\
21,1,85-87 \\
21,1,135-137 \\
21,2, \quad 8-10 \\
21,2, \quad 47-49\end{array}$ & $\begin{array}{l}185.16 \\
185.66 \\
186.16 \\
186.39 \\
186.78\end{array}$ & $\begin{array}{l}.28 \\
.19 \\
.29 \\
.96 \\
.26\end{array}$ & $\begin{array}{l}+70.8 \\
+83.8 \\
-56.5 \\
-50.4 \\
-5.7\end{array}$ & $\begin{array}{r}17.2 \\
129.1 \\
301.0 \\
334.5 \\
100.4\end{array}$ & $\begin{array}{l}-64.0 \\
-87.8 \\
-67.4 \\
-72.2 \\
-61.2\end{array}$ & $\begin{array}{r}332.9 \\
304.0 \\
326.4 \\
314.8 \\
76.5\end{array}$ & $\begin{array}{l}R \\
R \\
R \\
R \\
R\end{array}$ & $\begin{array}{l}8.7 \\
3.9 \\
8.5 \\
4.2\end{array}$ \\
\hline $\begin{array}{l}22,1,35-37 \\
22,1,81-83 \\
22,1,117-119 \\
22,2,32-34 \\
22,2,82-84 \\
22,3,35-37 \\
22,3,82-84 \\
22,3,135-137 \\
22,4,35-37 \\
22,4,82-84 \\
22,5,35-37 \\
22,5,82-84\end{array}$ & $\begin{array}{l}187.16 \\
187.62 \\
187.98 \\
188.63 \\
189.13 \\
190.16 \\
190.63 \\
191.16 \\
191.66 \\
192.13 \\
193.16 \\
193.63\end{array}$ & $\begin{array}{r}.18 \\
.11 \\
.11 \\
.28 \\
1.13 \\
6.68 \\
.09 \\
3.59 \\
.15 \\
.14 \\
.07 \\
.44\end{array}$ & $\begin{array}{r}-26.2 \\
+21.9 \\
-36.4 \\
+6.0 \\
-63.1 \\
-36.2 \\
+23.1 \\
+83.2 \\
-50.3 \\
+11.4 \\
-37.2 \\
-56.0\end{array}$ & $\begin{array}{r}71.6 \\
32.9 \\
121.4 \\
56.0 \\
36.7 \\
93.1 \\
53.9 \\
67.7 \\
155.5 \\
82.8 \\
63.3 \\
55.2\end{array}$ & $\begin{array}{l}-70.0 \\
-73.6 \\
-64.6 \\
-69.5 \\
-69.5 \\
-61.5 \\
-84.4 \\
-71.4 \\
-76.1 \\
-67.2 \\
-65.0 \\
-69.8\end{array}$ & $\begin{array}{r}130.2 \\
124.8 \\
134.0 \\
32.4 \\
4.1 \\
18.3 \\
1.2 \\
3.5 \\
39.1 \\
40.4 \\
19.3 \\
43.5\end{array}$ & $\begin{array}{l}R \\
R \\
R \\
R \\
R \\
R \\
R \\
R \\
R \\
R \\
R \\
R \\
R\end{array}$ & $\begin{array}{r}13.5 \\
38.9 \\
50.7 \\
5.9 \\
18.5 \\
49.8\end{array}$ \\
\hline $\begin{array}{l}23,1,41-43 \\
23,2,35-37 \\
23,2,85-87 \\
23,2,135-137 \\
23,3,35-37 \\
23,3,85-87 \\
23,3,135-137 \\
23,4,35-37 \\
23,4,83-85 \\
23,4,132-134 \\
23,5,35-37 \\
23,5,85-87 \\
23,5,133-135\end{array}$ & $\begin{array}{l}194.52 \\
195.96 \\
196.46 \\
196.96 \\
197.46 \\
197.96 \\
198.46 \\
198.96 \\
199.44 \\
199.93 \\
200.46 \\
200.96 \\
201.44\end{array}$ & $\begin{array}{l}.15 \\
.32 \\
.13 \\
.38 \\
.59 \\
.35 \\
3.48 \\
.23 \\
.07 \\
.08 \\
.03 \\
.22 \\
.04\end{array}$ & $\begin{array}{l}+66.7 \\
+51.5 \\
+26.7 \\
-31.8 \\
-72.0 \\
-60.7 \\
-64.0 \\
+11.2 \\
-44.5 \\
-29.0 \\
-58.5 \\
-23.3 \\
-47.4\end{array}$ & $\begin{array}{r}90.9 \\
61.0 \\
51.6 \\
112.5 \\
21.0 \\
14.0 \\
12.2 \\
136.2 \\
88.7 \\
53.4 \\
.3 \\
68.2 \\
16.8\end{array}$ & $\begin{array}{r}+69.8 \\
+45.7 \\
-9.1 \\
-50.0 \\
-74.3 \\
-67.6 \\
-65.0 \\
-70.8 \\
-75.3 \\
-64.1 \\
-75.7 \\
-61.7 \\
-69.3\end{array}$ & $\begin{array}{r}167.7 \\
44.5 \\
54.2 \\
108.3 \\
.5 \\
341.5 \\
359.9 \\
354.7 \\
332.1 \\
.4 \\
343.7 \\
342.9 \\
346.6\end{array}$ & $\begin{array}{l}\mathrm{N} \\
\mathrm{N} \\
\mathrm{R} \\
\mathrm{R} \\
\mathrm{R} \\
\mathrm{R} \\
\mathrm{R} \\
\mathrm{R} \\
\mathrm{R} \\
\mathrm{R} \\
\mathrm{R} \\
\mathrm{R}\end{array}$ & $\begin{array}{r}9.3 \\
42.7 \\
8.4\end{array}$ \\
\hline $\begin{array}{l}24,1,35-37 \\
24,1,85-87 \\
24,1,135-137 \\
24,2,35-37 \\
24,2,85-87 \\
24,3,35-37 \\
24,3,85-87 \\
24,3,135-137 \\
24,4,35-37\end{array}$ & $\begin{array}{l}201.46 \\
201.96 \\
202.46 \\
202.96 \\
203.46 \\
204.46 \\
204.96 \\
205.46 \\
205.96\end{array}$ & $\begin{array}{r}.11 \\
.24 \\
.12 \\
.82 \\
2.14 \\
.17 \\
.11 \\
.29 \\
.36\end{array}$ & $\begin{array}{r}-58.8 \\
+70.0 \\
+48.3 \\
-70.2 \\
-69.4 \\
+48.6 \\
+1.6 \\
+69.5 \\
+28.5\end{array}$ & $\begin{array}{r}95.0 \\
68.6 \\
22.4 \\
122.9 \\
41.8 \\
45.4 \\
73.1 \\
22.8 \\
56.3\end{array}$ & $\begin{array}{l}-67.2 \\
-67.1 \\
-70.4 \\
-76.7 \\
-70.9 \\
-75.7 \\
-68.1 \\
-72.1 \\
-76.9\end{array}$ & $\begin{array}{l}82.6 \\
43.9 \\
51.2 \\
55.1 \\
36.0 \\
51.1 \\
62.5 \\
51.2 \\
54.0\end{array}$ & $\begin{array}{l}R \\
R \\
R \\
R \\
R \\
R \\
R \\
R \\
R\end{array}$ & $\begin{array}{r}4.4 \\
36.7\end{array}$ \\
\hline $\begin{array}{l}25,1,30-32 \\
25,1,85-87\end{array}$ & $\begin{array}{l}206.31 \\
206.86\end{array}$ & $\begin{array}{l}.19 \\
.09\end{array}$ & $\begin{array}{r}+31.7 \\
-3.3\end{array}$ & $\begin{array}{l}37.9 \\
51.3\end{array}$ & $\begin{array}{l}-66.1 \\
-68.1\end{array}$ & $\begin{array}{l}34.1 \\
58.6\end{array}$ & $\begin{array}{l}\mathrm{R} \\
\mathrm{R}\end{array}$ & \\
\hline
\end{tabular}


Table A5 (continued).

\begin{tabular}{|c|c|c|c|c|c|c|c|c|}
\hline $\begin{array}{l}\text { Core, Section, } \\
\text { Interval }(\mathrm{cm})\end{array}$ & $\begin{array}{l}\text { Depth } \\
\text { (mbsf) }\end{array}$ & $\begin{array}{c}\mathrm{J} \\
\mathrm{NRM} \\
(\mathrm{mA} / \mathrm{m})\end{array}$ & $\begin{array}{l}\text { Incl. } \\
\text { NRM } \\
\text { (deg) }\end{array}$ & $\begin{array}{l}\text { Decl. } \\
\text { NRM } \\
\text { (deg) }\end{array}$ & $\begin{array}{c}\text { Incl } \\
\text { stable } \\
\text { (deg) }\end{array}$ & $\begin{array}{l}\text { Decl. } \\
\text { stable } \\
\text { (deg) }\end{array}$ & Pol. & $\begin{array}{l}\mathrm{MDF} \\
(\mathrm{mT})\end{array}$ \\
\hline $25,1,135-137$ & 207.36 & .11 & -41.6 & 37.3 & -67.8 & 24.7 & $\mathrm{R}$ & \\
\hline $25,2,30-32$ & 207.81 & .17 & +15.9 & 68.1 & -73.1 & 34.1 & $\mathrm{R}$ & \\
\hline $25,2,85-87$ & 208.36 & .20 & -6.4 & 48.4 & -68.2 & 64.5 & $\mathrm{R}$ & 48.2 \\
\hline $25,3,30-32$ & 209.31 & .18 & -17.9 & 53.2 & -63.1 & 44.8 & $\mathrm{R}$ & \\
\hline $26,1,35-37$ & 210.86 & .12 & +20.9 & 52.9 & -68.9 & 125.2 & $\mathrm{R}$ & \\
\hline $26,1,85-87$ & 211.36 & .15 & +25.5 & 65.5 & -63.6 & 127.4 & $\mathrm{R}$ & \\
\hline $26,1,135-137$ & 211.86 & .16 & -14.6 & 123.8 & -68.6 & 131.6 & $\mathrm{R}$ & \\
\hline $26,2,35-37$ & 212.36 & .66 & -68.9 & 126.1 & -77.0 & 125.5 & $\mathrm{R}$ & \\
\hline $26,2,85-87$ & 212.86 & 2.46 & -53.7 & 110.6 & -70.3 & 126.8 & $\mathrm{R}$ & \\
\hline $26,2,135-137$ & 213.36 & .89 & -49.0 & 111.4 & -63.9 & 112.8 & $\mathrm{R}$ & 48.0 \\
\hline $26,3,35-37$ & 213.86 & .06 & +11.4 & 62.9 & -70.7 & 118.5 & $\mathrm{R}$ & \\
\hline $27,1,85-87$ & 215.16 & .20 & -56.1 & 64.8 & -67.3 & 18.0 & $\mathrm{R}$ & 40.9 \\
\hline $27,1,115-117$ & 215.46 & .43 & -84.0 & 60.8 & -85.2 & 6.1 & $\mathrm{R}$ & 54.8 \\
\hline $28,1,35-37$ & 216.46 & .17 & -40.1 & 68.7 & -67.8 & 18.4 & $\mathrm{R}$ & 38.1 \\
\hline $28,1,85-87$ & 216.96 & 1.60 & -64.5 & 46.8 & -69.3 & 5.2 & $\mathrm{R}$ & 48.7 \\
\hline $28,1,135-137$ & 217.46 & 2.06 & -64.3 & 100.5 & -72.7 & 33.5 & $\mathrm{R}$ & 27.0 \\
\hline $28,2,35-37$ & 217.96 & 3.36 & -69.6 & 48.6 & -73.5 & 38.4 & $\mathrm{R}$ & 54.8 \\
\hline $28,2,85-87$ & 218.46 & 3.63 & -68.8 & 34.6 & -72.3 & 24.4 & $\mathrm{R}$ & \\
\hline $28,2,135-137$ & 218.96 & 2.35 & -71.3 & 54.6 & -67.4 & 25.5 & $\mathrm{R}$ & 49.5 \\
\hline $28,3,35-37$ & 219.46 & .35 & -76.9 & 72.1 & -85.3 & 37.3 & $\mathrm{R}$ & 4.1 \\
\hline $28,3,85-87$ & 219.96 & .14 & +36.9 & 52.3 & -70.2 & 27.2 & $\mathrm{R}$ & 7.9 \\
\hline $28,4,35-37$ & 220.96 & .24 & +37.7 & 79.9 & -64.0 & 63.5 & $\mathrm{R}$ & 6.8 \\
\hline $29,1,35-37$ & 221.46 & .28 & +27.4 & 96.4 & -65.2 & 110.6 & $\mathrm{R}$ & 9.5 \\
\hline $29,1,85-87$ & 221.96 & .12 & +36.5 & 81.7 & -64.2 & 82.2 & $\mathrm{R}$ & 4.1 \\
\hline $29,1,135-137$ & 222.46 & .13 & -74.0 & 165.2 & -74.4 & 114.4 & $\mathrm{R}$ & 3.3 \\
\hline $29,2,35-37$ & 222.96 & .19 & -31.2 & 106.2 & -75.6 & 136.0 & $\mathrm{R}$ & 13.6 \\
\hline $29,2,85-87$ & 223.46 & .14 & -51.0 & 37.7 & -61.1 & 106.6 & $\mathrm{R}$ & 12.5 \\
\hline $29,3,35-37$ & 224.46 & .66 & -6.5 & 117.0 & -66.0 & 109.6 & $\mathrm{R}$ & 44.4 \\
\hline $29,3,85-87$ & 224.96 & .48 & -33.4 & 112.0 & -67.4 & 95.4 & $\mathrm{R}$ & 33.2 \\
\hline $29,3,135-137$ & 225.46 & .14 & +70.1 & 56.2 & +78.5 & 274.7 & $\mathrm{~N}$ & 17.5 \\
\hline $30,1,35-37$ & 226.46 & .31 & +75.5 & 89.1 & +77.6 & 102.9 & $\mathrm{~N}$ & 6.6 \\
\hline $30,1,85-87$ & 226.96 & .98 & +72.8 & 201.8 & +79.0 & 116.5 & $\mathrm{~N}$ & 45.9 \\
\hline $30,1,135-137$ & 227.46 & .53 & +68.0 & 99.6 & +62.4 & 115.5 & $\mathrm{~N}$ & 26.2 \\
\hline $30,2,35-37$ & 227.96 & .39 & +55.8 & 99.8 & +64.5 & 127.3 & $\mathrm{~N}$ & 13.2 \\
\hline $30,2,85-87$ & 228.46 & .27 & +80.0 & 40.1 & +77.6 & 105.2 & $\mathrm{~N}$ & 6.3 \\
\hline $30,2,135-137$ & 228.96 & .43 & +68.6 & 116.6 & +65.4 & 106.9 & $\mathrm{~N}$ & 9.3 \\
\hline $30,3,35-37$ & 229.46 & .43 & +66.0 & 49.8 & +71.6 & 88.0 & $\mathrm{~N}$ & 11.8 \\
\hline $30,3,85-87$ & 229.96 & .47 & +78.7 & 146.0 & +76.9 & 117.9 & $\mathrm{~N}$ & 8.4 \\
\hline $30,3,135-137$ & 230.46 & 1.56 & +73.8 & 212.6 & +74.3 & 113.1 & $\mathrm{~N}$ & 44.2 \\
\hline $30,4,35-37$ & 230.96 & 1.08 & +75.9 & 151.5 & +67.1 & 123.8 & $\mathrm{~N}$ & 42.3 \\
\hline $30,4,85-87$ & 231.46 & 1.18 & +80.9 & 140.0 & +81.3 & 139.9 & $\mathrm{~N}$ & 37.0 \\
\hline $30,4,135-137$ & 231.96 & 2.55 & +87.5 & 193.4 & +74.3 & 109.3 & $\mathrm{~N}$ & 44.6 \\
\hline $31,1,35-37$ & 232.46 & 1.16 & -74.9 & 6.4 & -79.6 & 318.0 & & \\
\hline $31,1,85-87$ & 232.96 & 4.98 & +75.5 & 136.9 & +76.8 & 137.2 & $\mathrm{~N}$ & 47.2 \\
\hline
\end{tabular}


Table A5 (continued).

\begin{tabular}{|c|c|c|c|c|c|c|c|c|}
\hline $\begin{array}{l}\text { Core, Section, } \\
\text { Interval (cm) }\end{array}$ & $\begin{array}{l}\text { Depth } \\
\text { (mbsf) }\end{array}$ & $\begin{array}{c}\mathrm{J} \\
\mathrm{NRM} \\
(\mathrm{mA} / \mathrm{m})\end{array}$ & $\begin{array}{l}\text { Incl. } \\
\text { NRM } \\
\text { (deg) }\end{array}$ & $\begin{array}{l}\text { Decl. } \\
\text { NRM } \\
\text { (deg) }\end{array}$ & $\begin{array}{c}\text { Incl } \\
\text { stable } \\
\text { (deg) }\end{array}$ & $\begin{array}{c}\text { Decl. } \\
\text { stable } \\
\text { (deg) }\end{array}$ & Pol. & $\begin{array}{l}\text { MDF } \\
(\mathrm{mT})\end{array}$ \\
\hline $31,1,135-137$ & 233.46 & 7.89 & +75.5 & 158.0 & +75.4 & 159.1 & $\mathrm{~N}$ & 45.9 \\
\hline $31,2,35-37$ & 233.96 & .99 & +63.6 & 43.0 & +74.3 & 129.7 & $\mathrm{~N}$ & 43.1 \\
\hline $31,2,85-87$ & 234.46 & .32 & +68.8 & 118.5 & +66.0 & 125.5 & $\mathrm{~N}$ & 6.4 \\
\hline $31,2,135-137$ & 234.96 & .30 & +71.3 & 123.4 & +70.5 & 113.2 & $\mathrm{~N}$ & 9.7 \\
\hline $31,3,35-37$ & 235.46 & .41 & +58.5 & 89.5 & +66.1 & 140.2 & $\mathrm{~N}$ & 12.6 \\
\hline $31,3,85-87$ & 235.96 & .65 & +66.4 & 99.5 & +64.2 & 129.9 & $\mathrm{~N}$ & 13.2 \\
\hline $31,4,35-37$ & 236.96 & .63 & +77.7 & 100.5 & +69.8 & 131.0 & $\mathrm{~N}$ & 18.2 \\
\hline $31,4,85-87$ & 237.46 & .70 & +72.5 & 122.9 & +73.0 & 129.5 & $\mathrm{~N}$ & 13.4 \\
\hline $32,1,35-37$ & 238.46 & .89 & +64.8 & 93.5 & +70.8 & 111.9 & $\mathrm{~N}$ & 14.5 \\
\hline $32,1,85-87$ & 238.96 & 1.39 & +64.8 & 126.9 & +71.8 & 114.5 & $\mathrm{~N}$ & 16.1 \\
\hline $32,2,35-37$ & 239.96 & .47 & +61.5 & 123.6 & +62.5 & 121.8 & $\mathrm{~N}$ & 12.7 \\
\hline $32,2,85-87$ & 240.46 & .32 & +60.2 & 85.4 & +72.3 & 98.5 & $\mathrm{~N}$ & 12.7 \\
\hline $32,2,135-137$ & 240.96 & .51 & +81.1 & 122.2 & +80.5 & 108.4 & $\mathrm{~N}$ & 13.7 \\
\hline $32,3,35-37$ & 241.46 & .89 & +57.0 & 112.8 & +73.4 & 104.1 & $\mathrm{~N}$ & 10.6 \\
\hline $32,3,85-87$ & 241.96 & .62 & +81.7 & 203.0 & +70.0 & 123.0 & $\mathrm{~N}$ & 11.8 \\
\hline $32,3,135-137$ & 242.46 & .93 & +72.5 & 107.0 & +74.1 & 107.4 & $\mathrm{~N}$ & 15.0 \\
\hline $32,4,35-37$ & 242.96 & .55 & +65.7 & 122.4 & +65.5 & 120.3 & $\mathrm{~N}$ & 12.7 \\
\hline $32,4,85-87$ & 243.46 & .33 & +60.7 & 132.0 & +64.5 & 113.6 & $\mathrm{~N}$ & 17.6 \\
\hline $32,4,135-137$ & 243.96 & .64 & +70.3 & 100.4 & +68.5 & 105.9 & $\mathrm{~N}$ & 16.3 \\
\hline $33,1,135-137$ & 245.06 & .31 & +71.4 & 82.6 & +74.3 & & $\mathrm{~N}$ & 18.5 \\
\hline $33,2,35-37$ & 245.56 & .30 & +72.3 & 53.9 & +72.1 & 48.9 & $\mathrm{~N}$ & 15.6 \\
\hline $34,1,35-37$ & 246.3 & .82 & +69.8 & 77.6 & +72.1 & & $\mathrm{~N}$ & 19.1 \\
\hline $34,1,85-87$ & 246.86 & .5 & +55.5 & 63.9 & +77.2 & 75.7 & $\mathrm{~N}$ & 8.4 \\
\hline $34,1,135-137$ & 247.36 & .8 & +70.2 & 93.9 & +71.7 & 85.9 & $\mathrm{~N}$ & 15.2 \\
\hline $34,2,35-37$ & 247.86 & .64 & +52.8 & 104.5 & +66.0 & 88.6 & $\mathrm{~N}$ & 13.7 \\
\hline $34,2,85-87$ & 248.36 & 1.09 & +62.0 & 62.7 & +71.0 & 69.5 & $\mathrm{~N}$ & 28.9 \\
\hline $34,2,135-137$ & 248.86 & 2.27 & +67.8 & 126.2 & +80.9 & 94.1 & $\mathrm{~N}$ & 18.3 \\
\hline $34,3,23-25$ & 249.24 & .96 & +61.5 & 47.0 & +74.4 & 54.5 & $\mathrm{~N}$ & 19.8 \\
\hline $34,4,35-37$ & 250.86 & 2.88 & +77.4 & 19.3 & +78.2 & 18.5 & $\mathrm{~N}$ & 46.9 \\
\hline $34,4,85-87$ & 251.36 & 3.14 & +67.1 & 49.0 & +67.6 & 46.6 & $\mathrm{~N}$ & 47.9 \\
\hline $34,4,135-137$ & 251.86 & 1.48 & +72.7 & 51.9 & +70.7 & 49.9 & $\mathrm{~N}$ & 35.3 \\
\hline $34,5,35-37$ & 252.36 & .66 & +62.8 & 40.0 & +64.6 & 39.1 & $\mathrm{~N}$ & 19.5 \\
\hline $34,5,85-87$ & 252.86 & .46 & +70.1 & 40.4 & +68.6 & 32.8 & $\mathrm{~N}$ & 23.4 \\
\hline $34,5,135-137$ & 253.36 & .32 & +59.0 & 78.6 & +70.8 & 55.2 & $\mathrm{~N}$ & 14.8 \\
\hline $34,6,35-37$ & 253.86 & .43 & +65.3 & 72.6 & +71.8 & 22.7 & $\mathrm{~N}$ & 17.6 \\
\hline $34,6,85-87$ & 254.36 & .58 & +64.2 & 48.4 & +79.1 & 17.8 & $\mathrm{~N}$ & 15.6 \\
\hline $34,6,135-137$ & 254.86 & .50 & +77.9 & 61.9 & +75.7 & 21.1 & $\mathrm{~N}$ & 13.3 \\
\hline
\end{tabular}

$\mathrm{J}_{\mathrm{NRM}}$, Decl.NRM, Incl. NRM $_{\text {- intensity, inclination, declination of the natural remanent magnetization; Incl.stable, Decl.stable }}$ inclination, declination of the stable remanence after AF demagnetization; Pol., N, R - polarity, normal, reversed; MDF - median destructive field. 
Table A6. Paleomagnetic properties of samples from Hole 644B.

\begin{tabular}{|c|c|c|c|c|c|c|c|c|}
\hline $\begin{array}{l}\text { Core, Section, } \\
\text { Interval (cm) }\end{array}$ & $\begin{array}{l}\text { Depth } \\
\text { (mbsf) }\end{array}$ & $\begin{array}{c}\mathrm{J} \\
\mathrm{NRM} \\
(\mathrm{mA} / \mathrm{m})\end{array}$ & $\begin{array}{l}\text { Incl. } \\
\text { NRM } \\
\text { (deg) }\end{array}$ & $\begin{array}{l}\text { Decl. } \\
\text { NRM } \\
\text { (deg) }\end{array}$ & $\begin{array}{c}\text { Incl } \\
\text { stable } \\
\text { (deg) }\end{array}$ & $\begin{array}{l}\text { Decl. } \\
\text { stable } \\
\text { (deg) }\end{array}$ & Pol. & $\begin{array}{l}\text { MDF } \\
(\mathrm{mT})\end{array}$ \\
\hline $1,1,11-13$ & .12 & 40.43 & +80.9 & 322.2 & +81.2 & 312.9 & $\mathrm{~N}$ & 35.4 \\
\hline $1,1,72-74$ & .73 & 14.12 & +74.9 & 356.1 & +73.0 & 352.2 & $\mathrm{~N}$ & 22.5 \\
\hline $1,1,137-139$ & 1.38 & 7.03 & +77.6 & 328.4 & +74.0 & 314.1 & $\mathrm{~N}$ & 28.2 \\
\hline $1,2,45-47$ & 1.96 & 25.27 & +78.4 & 326.3 & +78.4 & 321.7 & $\mathrm{~N}$ & 27.0 \\
\hline $1,2,96-98$ & 2.47 & 11.07 & +77.9 & 32.5 & +78.3 & 16.9 & $\mathrm{~N}$ & 28.4 \\
\hline $1,2,136-138$ & 2.87 & 28.74 & +78.5 & 67.3 & +78.6 & 59.0 & $\mathrm{~N}$ & 28.0 \\
\hline $1,3,53-55$ & 3.54 & 39.01 & +56.3 & 57.7 & +76.3 & 16.1 & $\mathrm{~N}$ & 23.9 \\
\hline $1,3,112-114$ & 4.13 & 25.18 & +71.0 & 45.5 & +71.8 & 43.4 & $\mathrm{~N}$ & 29.6 \\
\hline $2,1,71-73$ & 5.32 & 47.00 & +71.0 & 39.3 & +72.2 & 28.1 & $\mathrm{~N}$ & 30.5 \\
\hline $2,1,115-117$ & 5.76 & 57.45 & +81.3 & 358.2 & +81.4 & 339.9 & $\mathrm{~N}$ & 31.3 \\
\hline $2,2,14-16$ & 6.25 & 38.97 & +76.8 & 315.8 & +75.3 & 314.9 & $\mathrm{~N}$ & 31.5 \\
\hline $2,2,71-73$ & 6.82 & 3.17 & +72.5 & 40.1 & +66.6 & 75.5 & $\mathrm{~N}$ & 21.8 \\
\hline $2,2,133-135$ & 7.44 & 8.07 & +75.1 & 46.6 & +80.1 & 26.3 & $\mathrm{~N}$ & 22.4 \\
\hline $2,3,31-33$ & 7.92 & 55.54 & +81.8 & 337.5 & +81.5 & 328.1 & $\mathrm{~N}$ & 29.6 \\
\hline $2,3,72-74$ & 8.33 & 48.77 & +81.5 & 358.5 & +77.5 & 358.9 & $\mathrm{~N}$ & 29.8 \\
\hline $2,3,138-140$ & 8.99 & 46.64 & +80.3 & 346.1 & +74.0 & 340.0 & $\mathrm{~N}$ & 28.5 \\
\hline $2,4,36-38$ & 9.47 & .98 & +78.9 & 83.3 & -61.7 & 156.6 & & 8.9 \\
\hline $2,4,92-94$ & 10.03 & 35.67 & +84.7 & 60.3 & +82.5 & 23.8 & $\mathrm{~N}$ & 22.9 \\
\hline $2,4,146-148$ & 10.57 & 50.41 & +77.4 & 303.3 & +77.8 & 309.8 & $\mathrm{~N}$ & 27.1 \\
\hline $2,5,37-39$ & 10.98 & 56.78 & +80.2 & 8.6 & +76.5 & 2.5 & $\mathrm{~N}$ & 25.8 \\
\hline $2,5,93-95$ & 11.54 & 31.56 & +86.6 & 10.8 & +87.1 & 51.0 & $\mathrm{~N}$ & 22.4 \\
\hline $2,5,136-138$ & 11.97 & 30.89 & +87.6 & 243.9 & +86.6 & 312.7 & $\mathrm{~N}$ & 23.9 \\
\hline $2,6,46-48$ & 12.57 & 21.62 & +79.0 & 1.2 & +79.0 & 359.1 & $\mathrm{~N}$ & 26.3 \\
\hline $2,6,92-94$ & 13.03 & 8.06 & +86.3 & 280.0 & +85.8 & 344.5 & $\mathrm{~N}$ & 25.9 \\
\hline $2,6,146-148$ & 13.57 & 21.74 & +70.6 & 4.7 & +68.7 & 359.2 & $\mathrm{~N}$ & 23.8 \\
\hline $2,7,17-19$ & 13.78 & 1.83 & +37.9 & 33.8 & -40.4 & 238.0 & & 38.4 \\
\hline $3,1,67-69$ & 14.78 & 71.98 & +73 & 50.2 & & & $\mathrm{~N}$ & 28.1 \\
\hline $3,1,111-113$ & 15.22 & 24. & +71 & 81 & +7 & 3 & $\mathrm{~N}$ & 26.3 \\
\hline $3,2,30-32$ & 15.91 & 26. & +82.9 & 39 & +79.8 & 23.7 & $\mathrm{~N}$ & 29.8 \\
\hline $3,2,91-93$ & 16.52 & 48.33 & +72.1 & 70.8 & +70.5 & 68.3 & $\mathrm{~N}$ & 28.1 \\
\hline $3,2,135-137$ & 16.96 & 44.79 & +66.1 & 128.5 & +67.4 & 133.4 & $\mathrm{~N}$ & 28.2 \\
\hline $3,3,45-47$ & 17.56 & 25.64 & +70.2 & 116.6 & +72.1 & 126.5 & $\mathrm{~N}$ & 17.4 \\
\hline $3,3,90-92$ & 18.01 & 13.37 & +71.5 & 140.6 & +72.9 & 142.9 & $\mathrm{~N}$ & 17.6 \\
\hline $3,3,134-136$ & 18.45 & 9.10 & +73.6 & 152.9 & +70.4 & 148.2 & $\mathrm{~N}$ & 12.1 \\
\hline $3,4,34-36$ & 18.95 & 50.71 & +76.1 & 160.2 & +75.8 & 162.8 & $\mathrm{~N}$ & 34.7 \\
\hline $3,4,91-93$ & 19.52 & 11.10 & +68.4 & 165.9 & +70.6 & 166.2 & $\mathrm{~N}$ & 18.7 \\
\hline $3,4,147-149$ & 20.08 & 70.69 & +77.3 & 197.1 & +79.2 & 189.1 & $\mathrm{~N}$ & 28.2 \\
\hline $3,5,44-46$ & 20.55 & 28.78 & +74.9 & 181.6 & +76.0 & 190.7 & $\mathrm{~N}$ & 23.0 \\
\hline $3,5,90-92$ & 21.01 & 29.83 & +77.3 & 150.4 & +80.0 & 162.8 & $\mathrm{~N}$ & 30.7 \\
\hline $3,5,135-137$ & 21.46 & 11.53 & +73.6 & 148.8 & +79.3 & 178.3 & $\mathrm{~N}$ & 27.2 \\
\hline $3,6,35-37$ & 21.96 & 16.03 & +85.8 & 74.0 & +89.0 & 169.3 & $\mathrm{~N}$ & 19.3 \\
\hline $3,6,92-94$ & 22.53 & 7.28 & +74.5 & 131.4 & -5.4 & 154.5 & & 28.3 \\
\hline $4,1,45-47$ & 24.06 & 23.89 & +77.4 & 280.3 & +74.2 & 307.6 & $\mathrm{~N}$ & 29.4 \\
\hline $4,1,90-92$ & 24.51 & 20.05 & +78.3 & 298.1 & +78.4 & 308.2 & $\mathrm{~N}$ & 25.0 \\
\hline $4,1,145-147$ & 25.06 & 16.50 & +74.1 & 292.0 & +72.6 & 312.2 & $\mathrm{~N}$ & 29.9 \\
\hline $4,2,34-36$ & 25.45 & 11.86 & +73.5 & 276.2 & +70.6 & 281.9 & $\mathrm{~N}$ & 27.7 \\
\hline
\end{tabular}


Table A6 (continued).

\begin{tabular}{|c|c|c|c|c|c|c|c|c|}
\hline $\begin{array}{l}\text { Core, Section, } \\
\text { Interval }(\mathrm{cm})\end{array}$ & $\begin{array}{l}\text { Depth } \\
\text { (mbsf) }\end{array}$ & $\begin{array}{c}\mathrm{J} \\
\mathrm{NRM} \\
(\mathrm{mA} / \mathrm{m})\end{array}$ & $\begin{array}{l}\text { Incl. } \\
\text { NRM } \\
\text { (deg) }\end{array}$ & $\begin{array}{l}\text { Decl. } \\
\text { NRM } \\
\text { (deg) }\end{array}$ & $\begin{array}{c}\text { Incl } \\
\text { stable } \\
\text { (deg) }\end{array}$ & $\begin{array}{c}\text { Decl. } \\
\text { stable } \\
\text { (deg) }\end{array}$ & Pol. & $\begin{array}{l}\text { MDF } \\
(\mathrm{mT})\end{array}$ \\
\hline $4,2,90-92$ & 26.01 & 11.73 & +73.2 & 28.7 & +67.2 & 6.6 & $\mathrm{~N}$ & 20.5 \\
\hline $4,2,145-147$ & 26.56 & 14.96 & +74.4 & 32.8 & +71.8 & 24.5 & $\mathrm{~N}$ & 20.9 \\
\hline $4,3,45-47$ & 27.06 & 25.08 & +52.4 & 319.1 & +53.2 & 327.2 & $\mathrm{~N}$ & 27.4 \\
\hline $4,3,91-93$ & 27.52 & 11.21 & +55.4 & 323.4 & +22.1 & 187.4 & $\mathrm{~N}$ & 22.3 \\
\hline $4,3,135-137$ & 27.96 & 9.92 & +68.0 & 29.4 & +68.1 & 17.0 & $\mathrm{~N}$ & 12.8 \\
\hline $4,4,45-47$ & 28.56 & 24.45 & +44.6 & 342.6 & +77.9 & 4.6 & $\mathrm{~N}$ & 25.2 \\
\hline $4,4,93-95$ & 29.04 & 20.66 & +47.5 & 333.6 & +78.4 & 25.6 & $\mathrm{~N}$ & 17.8 \\
\hline $4,4,146-148$ & 29.57 & 10.60 & +36.6 & 359.8 & -63.0 & 113.0 & & 25.1 \\
\hline $4,5,44-46$ & 30.05 & 23.26 & +81.6 & 9.6 & +79.3 & 4.0 & $\mathrm{~N}$ & 23.9 \\
\hline $4,5,89-91$ & 30.50 & 25.65 & +73.7 & 19.0 & +74.7 & 18.5 & $\mathrm{~N}$ & 26.4 \\
\hline $4,5,133-135$ & 30.94 & 7.53 & +83.7 & 24.0 & +81.0 & 20.1 & $\mathrm{~N}$ & 27.6 \\
\hline $4,6,33-35$ & 31.44 & 46.21 & +68.1 & 355.1 & +71.8 & 358.8 & $\mathrm{~N}$ & 28.2 \\
\hline $4,6,75-77$ & 31.86 & 107.03 & +74.4 & 166.8 & +77.7 & 192.0 & $\mathrm{~N}$ & 19.1 \\
\hline $5,1,54-56$ & 33.65 & 32.14 & +81.4 & 150.1 & +79.1 & 161.9 & $\mathrm{~N}$ & 19.1 \\
\hline $5,1,92-94$ & 34.03 & 39.71 & +73.1 & 151.4 & +74.0 & 166.5 & $\mathrm{~N}$ & 23.0 \\
\hline $5,1,146-148$ & 34.57 & 50.22 & +75.0 & 140.9 & +75.4 & 152.0 & $\mathrm{~N}$ & 30.6 \\
\hline $5,2,46-48$ & 35.07 & 103.36 & +58.5 & 136.0 & +71.4 & 145.5 & $\mathrm{~N}$ & 39.6 \\
\hline $5,2,92-94$ & 35.53 & 63.03 & +83.2 & 186.6 & +83.2 & 155.5 & $\mathrm{~N}$ & 27.7 \\
\hline $5,2,135-137$ & 35.96 & 11.96 & +77.6 & 218.5 & +76.9 & 185.8 & $\mathrm{~N}$ & 19.4 \\
\hline $5,3,36-38$ & 36.47 & .70 & +57.1 & 106.4 & -23.5 & 180.0 & & 13.5 \\
\hline $5,3,91-93$ & 37.02 & 7.08 & +68.9 & 158.2 & +69.5 & 157.9 & $\mathrm{~N}$ & 24.3 \\
\hline $5,3,135-137$ & 37.46 & 24.64 & +82.7 & 186.6 & +86.5 & 171.6 & $\mathrm{~N}$ & 27.6 \\
\hline $5,4,37-39$ & 37.98 & 28.88 & +86.7 & 164.7 & +86.4 & 180.8 & $\mathrm{~N}$ & 27.0 \\
\hline $5,4,93-95$ & 38.54 & 18.84 & +74.9 & 199.3 & +74.4 & 188.4 & $\mathrm{~N}$ & 28.2 \\
\hline $5,5,3-5$ & 39.14 & 12.43 & +60.6 & 218.6 & +66.8 & 228.0 & $\mathrm{~N}$ & 35.5 \\
\hline $6,1,46-48$ & 43.07 & 19.56 & +66.5 & 101.0 & +68.3 & 11 & $\mathrm{~N}$ & 29.0 \\
\hline $6,1,90-92$ & 43.51 & 40.88 & +42.8 & 93.2 & +72.0 & 101.1 & $\mathrm{~N}$ & 43.7 \\
\hline $6,1,136-138$ & 43.97 & 10.58 & +74.8 & 139.9 & +68.1 & 132.6 & $\mathrm{~N}$ & 20.7 \\
\hline $6,2,45-47$ & 44.56 & 23.97 & +78.9 & 99.5 & +78.6 & 102.0 & $\mathrm{~N}$ & \\
\hline $6,2,90-92$ & 45.01 & 21.20 & +63.7 & 81.4 & +69.1 & 90.8 & $\mathrm{~N}$ & 63.7 \\
\hline $6,2,136-138$ & 45.47 & 13.89 & +66.5 & 93.9 & +66.5 & 94.3 & $\mathrm{~N}$ & 24.0 \\
\hline $6,3,46-48$ & 46.07 & 4.47 & +64.5 & 109.3 & +72.3 & 104.5 & $\mathrm{~N}$ & 10.7 \\
\hline $6,3,89-91$ & 46.50 & 7.19 & +64.3 & 97.0 & +68.1 & 91.8 & $\mathrm{~N}$ & 23.1 \\
\hline $6,3,136-138$ & 46.97 & 1.01 & +62.5 & 105.6 & +70.1 & 108.0 & $\mathrm{~N}$ & 4.3 \\
\hline $6,4,46-48$ & 47.57 & 41.69 & +66.7 & 71.6 & +69.4 & 78.8 & $\mathrm{~N}$ & 24.7 \\
\hline $6,4,90-92$ & 48.01 & 3.63 & +61.0 & 112.6 & +71.8 & 108.0 & $\mathrm{~N}$ & 36.5 \\
\hline $6,4,137-139$ & 48.48 & 21.23 & +74.4 & 92.4 & +73.2 & 89.1 & $\mathrm{~N}$ & 23.4 \\
\hline $6,5,45-47$ & 49.06 & 15.29 & +55.6 & 71.4 & +72.8 & 79.1 & $\mathrm{~N}$ & 23.7 \\
\hline $6,5,89-91$ & 49.50 & 22.53 & +69.7 & 96.8 & +71.4 & 98.9 & $\mathrm{~N}$ & 25.7 \\
\hline $6,5,133-135$ & 49.94 & 10.24 & +71.6 & 99.7 & +73.1 & 82.0 & $N$ & 13.3 \\
\hline $6,6,12-14$ & 50.23 & 9.79 & +71.4 & 314.4 & +69.5 & 310.1 & $\mathrm{~N}$ & 20.4 \\
\hline $7,1,36-38$ & 52.47 & 21.83 & +76.7 & 128.8 & +73.9 & 128.4 & $\mathrm{~N}$ & 22.3 \\
\hline $7,1,91-93$ & 53.02 & 2.20 & +40.9 & 79.7 & +72.5 & 118.6 & $\mathrm{~N}$ & 26.3 \\
\hline $7,1,136-138$ & 53.47 & 42.56 & +65.6 & 156.1 & +69.8 & 128.9 & $\mathrm{~N}$ & 18.2 \\
\hline $7,2,37-39$ & 53.98 & 72.29 & +76.3 & 92.3 & +77.8 & 91.7 & $\mathrm{~N}$ & 25.8 \\
\hline $7,2,90-92$ & 54.51 & 58.49 & +77.9 & 94.5 & +75.0 & 102.7 & $\mathrm{~N}$ & 13.9 \\
\hline $7,2,136-138$ & 54.97 & 55.34 & -12.8 & 198.7 & +83.4 & 137.2 & $\mathrm{~N}$ & 29.2 \\
\hline
\end{tabular}




\begin{tabular}{|c|c|c|c|c|c|c|c|c|}
\hline $\begin{array}{l}\text { Core, Section, } \\
\text { Interval (cm) }\end{array}$ & $\begin{array}{l}\text { Depth } \\
\text { (mbsf) }\end{array}$ & $\begin{array}{c}\mathrm{J} \\
\mathrm{NRM} \\
(\mathrm{mA} / \mathrm{m})\end{array}$ & $\begin{array}{l}\text { Incl. } \\
\text { NRM } \\
\text { (deg) }\end{array}$ & $\begin{array}{l}\text { Decl. } \\
\text { NRM } \\
\text { (deg) }\end{array}$ & $\begin{array}{c}\text { Incl } \\
\text { stable } \\
\text { (deg) }\end{array}$ & $\begin{array}{l}\text { Decl. } \\
\text { stable } \\
\text { (deg) }\end{array}$ & Pol. & $\begin{array}{l}\text { MDF } \\
(\mathrm{mT})\end{array}$ \\
\hline $7,3,37-39$ & 55.48 & 13.03 & +65.8 & 129.7 & +69.3 & 121.0 & $\mathrm{~N}$ & 20.8 \\
\hline $7,3,89-91$ & 56.00 & 1.63 & +72.2 & 118.5 & +77.0 & 93.6 & $\mathrm{~N}$ & 4.6 \\
\hline $7,3,135-137$ & 56.46 & 1.22 & +75.6 & 149.7 & +69.3 & 120.9 & $\mathrm{~N}$ & 4.1 \\
\hline $7,4,37-39$ & 56.98 & 27.04 & +72.4 & 104.9 & +74.8 & 107.6 & $\mathrm{~N}$ & 38.0 \\
\hline $7,4,90-92$ & 57.51 & 10.33 & +66.0 & 110.1 & +67.4 & 124.9 & $\mathrm{~N}$ & 27.4 \\
\hline $7,4,134-136$ & 57.95 & 75.91 & +70.6 & 108.3 & +72.0 & 113.7 & $\mathrm{~N}$ & \\
\hline $7,5,8-10$ & 58.19 & 12.08 & +77.2 & 72.0 & +82.5 & 93.1 & $\mathrm{~N}$ & 33.5 \\
\hline $8,1,46-48$ & 62.07 & 12.42 & +78.8 & 247.5 & +74.1 & 265.1 & $\mathrm{~N}$ & 43.1 \\
\hline $8,1,89-91$ & 62.50 & 22.06 & +72.0 & 248.2 & +72.8 & 251.8 & $\mathrm{~N}$ & 29.2 \\
\hline $8,1,136-138$ & 62.97 & .59 & +71.7 & 209.4 & +69.2 & 224.0 & $\mathrm{~N}$ & 54.0 \\
\hline $8,2,45-47$ & 63.56 & 2.82 & +31.7 & 147.7 & +79.2 & 218.6 & $\mathrm{~N}$ & \\
\hline $8,2,89-91$ & 64.00 & 4.48 & +74.8 & 180.8 & +75.5 & 182.9 & $\mathrm{~N}$ & 27.6 \\
\hline $8,2,135-137$ & 64.46 & 11.01 & +50.3 & 204.9 & +69.4 & 219.4 & $\mathrm{~N}$ & 34.9 \\
\hline $8,3,45-47$ & 65.06 & 8.66 & +59.0 & 196.3 & +64.4 & 209.0 & $\mathrm{~N}$ & 34.6 \\
\hline $8,3,89-91$ & 65.50 & 46.38 & +64.7 & 190.4 & +68.6 & 197.4 & $\mathrm{~N}$ & 34.1 \\
\hline $8,3,134-136$ & 65.95 & 14.46 & +72.4 & 203.1 & +72.9 & 201.5 & $\mathrm{~N}$ & 30.9 \\
\hline $8,4,45-47$ & 66.56 & 6.02 & +65.6 & 200.7 & +67.0 & 203.0 & $\mathrm{~N}$ & 29.0 \\
\hline $8,4,91-93$ & 67.02 & 14.44 & +53.7 & 171.3 & +64.5 & 185.3 & $\mathrm{~N}$ & 23.2 \\
\hline $8,4,129-131$ & 67.40 & 186.44 & +72.6 & 153.4 & +75.6 & 197.1 & $\mathrm{~N}$ & 6.0 \\
\hline $8,5,20-22$ & 67.81 & 9.58 & +70.0 & .1 & +73.2 & 39.5 & $\mathrm{~N}$ & 27.7 \\
\hline $9,1,46-48$ & 71.57 & 30. & +18 & & & & $\mathrm{~N}$ & 4.7 \\
\hline $9,1,91-93$ & 72.02 & 39. & +74 & 19 & & 7.5 & $\mathrm{~N}$ & 19.9 \\
\hline $3,1,136-138$ & 72. & 14. & +77.8 & 98 & & 7.5 & $\mathrm{~N}$ & 3.1 \\
\hline $9,2,46-48$ & 73. & 90. & + & 5 & & 9 & $\mathrm{~N}$ & 25.1 \\
\hline $9,2,91-93$ & 73.52 & 9. & +79 & 59.8 & +7 & 85.5 & $\mathrm{~N}$ & 12.5 \\
\hline $9,2,136-138$ & 73. & 15. & + & 11 & + & 85 & $\mathrm{~N}$ & 14.7 \\
\hline $9,3,46-48$ & 74.57 & 7.64 & & 99.5 & & 83.2 & $\mathrm{~N}$ & 18.3 \\
\hline $9,3,90-92$ & 75.01 & 26.04 & +73.1 & 189.0 & +72.4 & 67.2 & $\mathrm{~N}$ & 3.1 \\
\hline $9,3,136-138$ & 75.47 & 4.39 & +67.9 & 21.5 & +7 & 51.4 & $\mathrm{~N}$ & 19.2 \\
\hline $9,4,46-48$ & 76.07 & 5.97 & +22.2 & 260.6 & +84.6 & 71.4 & $\mathrm{~N}$ & 13.7 \\
\hline $9,4,90-92$ & 76.51 & 27.41 & +72.0 & 60.6 & +73.3 & 57.0 & $\mathrm{~N}$ & 16.7 \\
\hline $9,4,135-137$ & 76.96 & 102.08 & +79.8 & 90.2 & +78.3 & 65.2 & $\mathrm{~N}$ & 25.9 \\
\hline $9,5,24-26$ & 77.35 & 12.52 & +84.9 & 144.0 & +84.5 & 38.4 & $\mathrm{~N}$ & 17.5 \\
\hline $10,1,46-48$ & 81.07 & 2.69 & +66.8 & 120.8 & +7 & 14 & $\mathrm{~N}$ & 7.8 \\
\hline $10,1,91-93$ & 81.52 & 11.24 & +82.2 & 297.0 & +87.5 & 224.7 & $\mathrm{~N}$ & 35.0 \\
\hline $10,1,137-139$ & 81.98 & 9.80 & +50.0 & 185.2 & +72.0 & 212.2 & $\mathrm{~N}$ & 51.9 \\
\hline $10,2,45-47$ & 82.56 & 1.83 & +45.6 & 120.9 & +77.3 & 144.9 & $\mathrm{~N}$ & 6.4 \\
\hline $10,2,90-92$ & 83.01 & 13.53 & -70.7 & 69.6 & -67.1 & 57.5 & $\mathrm{R}$ & 30.8 \\
\hline $10,2,145-147$ & 83.56 & 22.15 & -76.6 & 93.4 & -77.2 & 71.0 & $\mathrm{R}$ & 27.4 \\
\hline $10,3,46-48$ & 84.07 & 38.83 & -55.4 & 83.0 & -66.8 & 78.8 & $\mathrm{R}$ & 28.4 \\
\hline $10,3,90-92$ & 84.51 & 1.47 & -19.1 & 123.8 & -68.4 & 34.4 & $\mathrm{R}$ & 22.4 \\
\hline $10,3,136-138$ & 84.97 & .11 & -58.1 & 222.9 & -75.1 & 62.9 & $\mathrm{R}$ & \\
\hline $10,4,46-48$ & 85.57 & 4.78 & -69.0 & 16.5 & -77.5 & 39.5 & $\mathrm{R}$ & 28.9 \\
\hline $10,4,90-92$ & 86.01 & 8.65 & -50.7 & 74.2 & -69.8 & 76.9 & $\mathrm{R}$ & 28.8 \\
\hline $10,4,136-138$ & 86.47 & 13.07 & -64.5 & 52.9 & -71.6 & 40.3 & $\mathrm{R}$ & 28.1 \\
\hline $10,5,45-47$ & 87.06 & 12.20 & -56.6 & 64.2 & -71.0 & 50.5 & $\mathrm{R}$ & 29.8 \\
\hline $10,5,90-92$ & 87.51 & 3.76 & -43.7 & 121.0 & -68.1 & 97.8 & $\mathrm{R}$ & 35.0 \\
\hline
\end{tabular}


Table A6 (continued).

\begin{tabular}{|c|c|c|c|c|c|c|c|c|}
\hline $\begin{array}{l}\text { Core, Section, } \\
\text { Interval }(\mathrm{cm})\end{array}$ & $\begin{array}{l}\text { Depth } \\
\text { (mbsf) }\end{array}$ & $\begin{array}{c}\mathrm{J} \\
\mathrm{NRM} \\
(\mathrm{mA} / \mathrm{m})\end{array}$ & $\begin{array}{l}\text { Incl. } \\
\text { NRM } \\
\text { (deg) }\end{array}$ & $\begin{array}{l}\text { Decl. } \\
\text { NRM } \\
\text { (deg) }\end{array}$ & $\begin{array}{l}\text { Incl } \\
\text { stable } \\
\text { (deg) }\end{array}$ & $\begin{array}{l}\text { Decl. } \\
\text { stable } \\
\text { (deg) }\end{array}$ & Pol. & $\begin{array}{l}\text { MDF } \\
(\mathrm{mT})\end{array}$ \\
\hline $10,5,129-131$ & 87.90 & 1.38 & -25.8 & 27.1 & -75.3 & 39.7 & $\mathrm{R}$ & \\
\hline $11,1,56-58$ & 90.67 & 1.60 & +54.3 & 108.1 & -84.9 & 6.0 & $\mathrm{R}$ & 47.2 \\
\hline $11,1,92-94$ & 91.03 & 2.15 & +75.8 & 57.4 & -74.3 & 306.0 & $\mathrm{R}$ & 3.5 \\
\hline $11,1,137-139$ & 91.48 & 4.96 & +83.6 & 77.0 & -62.3 & 35.0 & $\mathrm{R}$ & 4.1 \\
\hline $11,2,45-47$ & 92.06 & 3.55 & +86.7 & 56.2 & -70.2 & 331.1 & $\mathrm{R}$ & 3.7 \\
\hline $11,2,91-93$ & 92.52 & 3.93 & +79.4 & 7.7 & -77.9 & 297.7 & $\mathrm{R}$ & 3.4 \\
\hline $11,2,136-138$ & 92.97 & .93 & +14.3 & 69.1 & -79.8 & 330.4 & $\mathrm{R}$ & 58.1 \\
\hline $11,3,47-49$ & 93.58 & 12.92 & -69.7 & 313.0 & -72.4 & 295.5 & $R$ & 29.3 \\
\hline $11,3,91-93$ & 94.02 & 1.61 & -70.8 & 41.3 & -79.6 & 311.2 & $\mathrm{R}$ & 34.8 \\
\hline $11,3,137-139$ & 94.48 & 1.83 & -80.1 & 144.9 & -82.7 & 303.6 & $R$ & \\
\hline $11,4,32-34$ & 94.93 & .62 & +60.4 & 64.5 & -66.4 & 300.4 & $\mathrm{R}$ & 34.5 \\
\hline $11,4,74-76$ & 95.35 & .21 & +19.6 & 171.1 & -72.0 & 325.7 & $\mathrm{R}$ & \\
\hline $12,1,46-48$ & 95.97 & .54 & -45.7 & 77.5 & -72.0 & 52.9 & $\mathrm{R}$ & 37.8 \\
\hline $12,1,90-92$ & 96.41 & 13.01 & -56.7 & 56.2 & -64.8 & 53.0 & $\mathrm{R}$ & 61.2 \\
\hline $12,1,136-138$ & 96.87 & 7.84 & -36.1 & 90.9 & -69.3 & 55.8 & $R$ & 24.1 \\
\hline $12,2,30-32$ & 97.31 & 12.05 & -64.6 & 29.3 & -66.7 & 17.1 & $\mathrm{R}$ & 24.8 \\
\hline $12,2,97-99$ & 97.98 & 28.99 & -68.9 & 73.5 & -71.8 & 75.7 & $\mathrm{R}$ & 25.1 \\
\hline $12,2,127-129$ & 98.28 & 19.50 & -71.4 & 74.4 & -72.1 & 66.9 & $\mathrm{R}$ & 18.3 \\
\hline $12,3,29-31$ & 98.80 & 24.07 & -58.7 & 51.9 & -68.3 & 49.4 & $\mathrm{R}$ & 20.0 \\
\hline $13,1,82-84$ & 53 & 21.60 & +76.0 & 96.2 & .7 & 87.3 & $\mathrm{~N}$ & 17.5 \\
\hline $13,1,147-149$ & 107.18 & 27.26 & +67.8 & 67.9 & 1 & 63.6 & $\mathrm{~N}$ & 16.7 \\
\hline $13,2,35-37$ & 107.56 & 27.45 & +71.4 & 20.6 & +68.0 & 27.9 & $\mathrm{~N}$ & 21.3 \\
\hline $13,2,90-92$ & 108.11 & 8.16 & +75.0 & 28.0 & +72.5 & 21.2 & $\mathrm{~N}$ & 11.8 \\
\hline $13,2,135-137$ & 108.56 & .46 & +68.1 & 112.9 & +5.3 & 60.1 & & 4.3 \\
\hline $13,3,45-47$ & 109.16 & .18 & +49.5 & 3.7 & -58.1 & 149.2 & $\mathrm{R}$ & 57.5 \\
\hline $13,3,90-92$ & 109.61 & .18 & -29.4 & 111.6 & -75.9 & 160.6 & $\mathrm{R}$ & \\
\hline $13,3,137-139$ & 110.08 & 2.26 & -34.0 & 133.4 & -73.2 & 185.5 & $\mathrm{R}$ & 39.5 \\
\hline $13,4,29-31$ & 110.50 & 17.32 & -69.3 & 119.9 & -79.4 & 144.0 & $\mathrm{R}$ & 33.3 \\
\hline $13,4,89-91$ & 111.10 & 1.63 & +56.7 & 100.9 & -66.9 & 147.6 & $\mathrm{R}$ & 3.8 \\
\hline $13,4,109-111$ & 111.30 & .78 & -1.5 & 97.2 & -69.8 & 56.8 & $\mathrm{R}$ & 17.7 \\
\hline $14,1,116-118$ & & 1.17 & +28.6 & & & & $\mathrm{R}$ & 8.6 \\
\hline $14,2,34-36$ & 113.25 & 2.22 & +30.0 & 131.7 & -60 & 22.6 & $\mathrm{R}$ & 4.0 \\
\hline $14,2,85-87$ & 113.76 & 2.19 & +28.7 & 156.2 & -73.4 & 37.3 & $\mathrm{R}$ & 4.2 \\
\hline $14,2,136-138$ & 114.27 & 4.71 & +16.3 & 113.2 & -71.6 & 25.5 & $\mathrm{R}$ & 4.4 \\
\hline $14,3,30-32$ & 114.71 & 7.73 & -60.8 & 33.8 & -70.3 & 31.3 & $\mathrm{R}$ & 5.9 \\
\hline $14,3,93-95$ & 115.34 & 1.26 & +76.9 & 110.4 & -69.6 & 12.1 & $\mathrm{R}$ & 4.8 \\
\hline $14,3,136-138$ & 115.77 & 5.09 & -14.4 & 48.4 & -72.6 & 9.6 & $\mathrm{R}$ & 32.1 \\
\hline $14,4,46-48$ & 116.37 & 12.98 & -59.3 & 30.1 & -69.0 & 26.7 & $\mathrm{R}$ & 33.6 \\
\hline $14,4,94-96$ & 116.85 & 23.91 & -62.9 & 9.5 & -63.5 & 12.3 & $\mathrm{R}$ & 28.0 \\
\hline $14,4,136-138$ & 117.27 & 11.44 & -70.6 & 17.0 & -72.3 & 4.1 & $\mathrm{R}$ & 21.2 \\
\hline $14,5,37-39$ & 117.78 & .23 & +7.7 & 102.9 & -70.0 & 34.5 & $\mathrm{R}$ & \\
\hline $14,5,88-90$ & 118.29 & .63 & -72.9 & 62.3 & -71.1 & 64.3 & $\mathrm{R}$ & 14.6 \\
\hline $14,5,135-137$ & 118.76 & 2.62 & +22.3 & 131.1 & -75.1 & 40.1 & $\mathrm{R}$ & 7.7 \\
\hline $14,6,28-30$ & 119.19 & 8.29 & -83.9 & 323.0 & -67.5 & 294.0 & $\mathrm{R}$ & 34.1 \\
\hline $15,1,46-48$ & 119.67 & 8.53 & +76.2 & 128.6 & -69.6 & 8.5 & $\mathrm{R}$ & 7.0 \\
\hline
\end{tabular}


Table A6 (continued).

\begin{tabular}{|c|c|c|c|c|c|c|c|c|}
\hline $\begin{array}{l}\text { Core, Section, } \\
\text { Interval }(\mathrm{cm})\end{array}$ & $\begin{array}{l}\text { Depth } \\
\text { (mbsf) }\end{array}$ & $\begin{array}{c}\mathrm{J} \\
\mathrm{NRM} \\
(\mathrm{mA} / \mathrm{m})\end{array}$ & $\begin{array}{l}\text { Incl. } \\
\text { NRM } \\
\text { (deg) }\end{array}$ & $\begin{array}{l}\text { Decl. } \\
\text { NRM } \\
\text { (deg) }\end{array}$ & $\begin{array}{c}\text { Incl } \\
\text { stable } \\
\text { (deg) }\end{array}$ & $\begin{array}{l}\text { Decl. } \\
\text { stable } \\
\text { (deg) }\end{array}$ & Pol. & $\begin{array}{l}\text { MDF } \\
(\mathrm{mT})\end{array}$ \\
\hline $15,1,91-93$ & 120.12 & 4.39 & -59.2 & 77.2 & -80.3 & 19.4 & $\mathrm{R}$ & 49.2 \\
\hline $15,1,136-138$ & 120.57 & 1.91 & +44.9 & 99.2 & -73.7 & 56.9 & $\mathrm{R}$ & 43.7 \\
\hline $15,2,46-48$ & 121.17 & 12.76 & -78.2 & 35.1 & -72.1 & 47.8 & $\mathrm{R}$ & 11.5 \\
\hline $15,2,90-92$ & 121.61 & 12.40 & -24.0 & 31.9 & -66.6 & 60.1 & $\mathrm{R}$ & 31.6 \\
\hline $15,2,136-138$ & 122.07 & 10.24 & -30.0 & 48.9 & -66.0 & 27.0 & $\mathrm{R}$ & 34.7 \\
\hline $15,3,36-38$ & 122.57 & 1.72 & -6.6 & 54.5 & -67.7 & 28.4 & $\mathrm{R}$ & 46.6 \\
\hline $15,3,91-93$ & 123.12 & 2.17 & +8.4 & 60.6 & -72.9 & 58.2 & $\mathrm{R}$ & 42.9 \\
\hline $15,3,146-148$ & 123.67 & 6.35 & -17.9 & 15.7 & -70.3 & 20.0 & $\mathrm{R}$ & 34.9 \\
\hline $15,4,46-48$ & 124.17 & 3.88 & +65.6 & 107.7 & -71.2 & 54.7 & $\mathrm{R}$ & 4.5 \\
\hline $15,4,91-93$ & 124.62 & 15.30 & +79.8 & 198.7 & +75.4 & 217.7 & & 18.4 \\
\hline $15,4,147-149$ & 125.18 & 7.23 & +86.1 & 164.7 & -65.7 & 10.2 & $\mathrm{R}$ & 4.0 \\
\hline $15,5,45-47$ & 125.66 & 7.87 & -53.3 & 41.2 & -68.4 & 38.2 & $\mathrm{R}$ & 37.8 \\
\hline $15,5,91-93$ & 126.12 & 4.55 & -37.4 & 24.9 & -62.9 & 19.4 & $R$ & 35.1 \\
\hline $15,5,136-138$ & 126.57 & 1.32 & +55.9 & 71.9 & -71.4 & 29.3 & $\mathrm{R}$ & 4.0 \\
\hline $15,6,46-48$ & 127.17 & .59 & +34.4 & 58.0 & -64.9 & 24.1 & $\mathrm{R}$ & 48.8 \\
\hline
\end{tabular}

$\mathrm{J}_{\mathrm{NRM}}$, Decl.NRM, Incl.NRM - intensity, inclination, declination of the natural remanent magnetization; Incl.stable, Decl.stable inclination, declination of the stable remanence after AF demagnetization; Pol., N, R - polarity, normal, reversed; MDF - median destructive field. 